\title{
A DFT Study on Rh-Catalyzed Asymmetric Dearomatization of 2-Naphthols Initiated with C-H Activation: A Refined Reaction Mechanism and Origins of Multiple Selectivity
}

Chao Zheng,* Jun Zheng, and Shu-Li You*

State Key Laboratory of Organometallic Chemistry, Shanghai Institute of Organic Chemistry, Chinese Academy of Sciences, 345 Lingling Lu, Shanghai 200032, China

E-mail: zhengchao@sioc.ac.cn or $\underline{\text { slyou@ sioc.ac.cn }}$

\section{Table of Contents}

1. The thermodynamics of the dearomatized product and the $\mathrm{O}$-alkenylation product.............S2

2. The structures of the four diastereomeric transition states for the $\mathrm{C}-\mathrm{H}$ cleavage step...........S3

3. The structures of the transition states for the interconversion of diastereomers of INT-5.......S4

4. The intrinsic reaction coordinate (IRC) analyses of TS-3 and TS-6- $\left(S_{\mathrm{a}}, R_{\mathrm{Rh}}\right) \ldots \ldots \ldots \ldots \ldots \ldots \ldots . . . \mathrm{S} 5$

5. The structures of the transition states for the migratory insertion with Rh-catalyst K2 $\ldots \ldots \ldots . . . \mathrm{S} 6$

6. The reduced density gradient (RDG) analyses of TS-6'-A and TS-7'-A ........................ 7

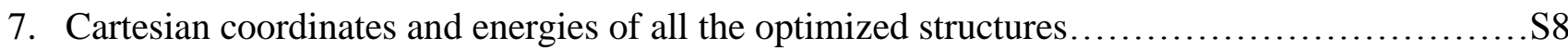


1. The thermodynamics of the dearomatized product and the O-alkenylation product

The relative energies of the dearomatized product $\mathbf{3}$ and the experimentally not observed O-alkenylation product $\mathbf{3}^{\prime}$ are calculated (Figure S1). The energy of $\mathbf{3}^{\prime}$ is higher than that of $\mathbf{3}$ by $3.8 \mathrm{kcal} / \mathrm{mol}$, probably due to highly strained seven-membered ring of $3^{\prime}$. This result, together with the very high energetic barrier of the transition state (TS-9, Figure 4 in the main text) leading to $\mathbf{3}^{\prime}$, demonstrates that the dearomatization process is both thermodynamically and kinetically favorable.

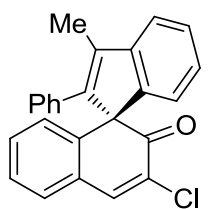

3

0.0

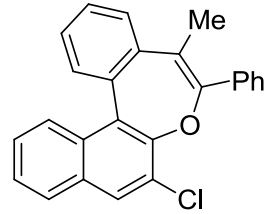

3'

3.8

Figure S1. The structures and the relative energies of the dearomatized product and O-alkenylation product 3 '. The $\Delta G$ values are in $\mathrm{kcal} / \mathrm{mol}$. 
2. The structures of the four diastereomeric transition states for the $\mathrm{C}-\mathrm{H}$ cleavage step

The structures and the relative energies of the four diastereomeric transition states for the $\mathrm{C}-\mathrm{H}$ cleavage step are shown in Figure S2. They are named according to: (1) the configuration of the axial chirality of the 1-phenyl-2-naphthol moiety $\left(R_{\mathrm{a}}\right.$ or $\left.S_{\mathrm{a}}\right)$; (2) the configuration of the central chirality of the Rh center $\left(R_{\mathrm{Re}}\right.$ or $\left.S_{\mathrm{Re}}\right)$; (3) the relative position of acetate ligand (Back or Front). The one with the lowest energy (TS-3- $\left(R_{\mathrm{a}}, S_{\mathrm{Rh}}\right)$-Back) are presented in Figure 2 of the main text as TS-3. This four diastereomers of TS-3 can lead to the four diastereomers of INT-5 directly after neutralization of the formed acetic acid (For example, TS-3- $\left(R_{\mathrm{a}}, S_{\mathrm{Rh}}\right)$-Back to INT-5- $\left(R_{\mathrm{a}}, R e_{\mathrm{Rh}}\right)$; TS-3- $\left(S_{\mathrm{a}}, R_{\mathrm{Rh}}\right)$-Back to INT5-( $\left.\left(S_{\mathrm{a}}, S i_{\mathrm{Rh}}\right)\right)$. Since we have proved that the diastereomers of INT-5 can interconvert to each other freely under the reaction conditions and the enantioselectivity is determined during the following migratory insertion step (Figures 3 and 4 in the main text), the central chirality established at the Rh center in TS-3 is irrelevant to enantioselectivity of the reaction.

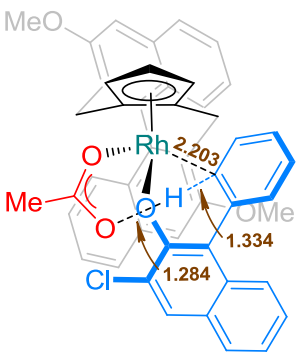

TS-3- $\left(R_{\mathrm{a}}, \mathrm{S}_{\mathrm{Rh}}\right)$-Back

$\mathbf{0 . 0}$

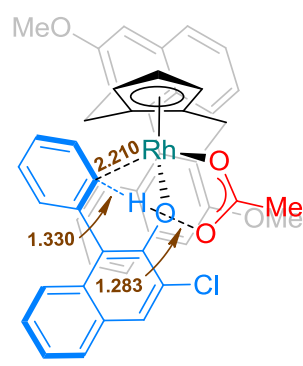

TS-3- $\left(R_{\mathrm{a}}, S_{\mathrm{Rh}}\right)$-Front

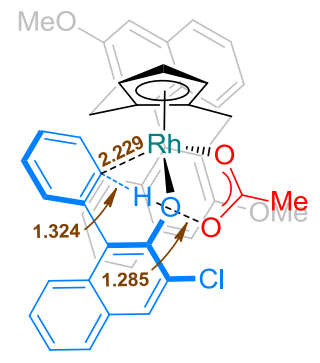

TS-3- $\left(S_{\mathrm{a}}, R_{\mathrm{Rh}}\right)$-Back

4.6

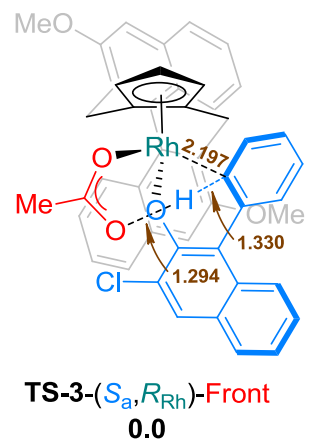

Figure S2. The structures and the relative energies of the four diastereomeric transition states for the $\mathrm{C}-\mathrm{H}$ cleavage step. The $\Delta G$ values are in $\mathrm{kcal} / \mathrm{mol}$ and the bond distances are in angstrom. 
3. The structures of the transition states for the interconversion of diastereomers of INT-5

The structures and the relative energies of the four transition states for the interconversion of diastereomers of INT-5 are shown in Figure S3.
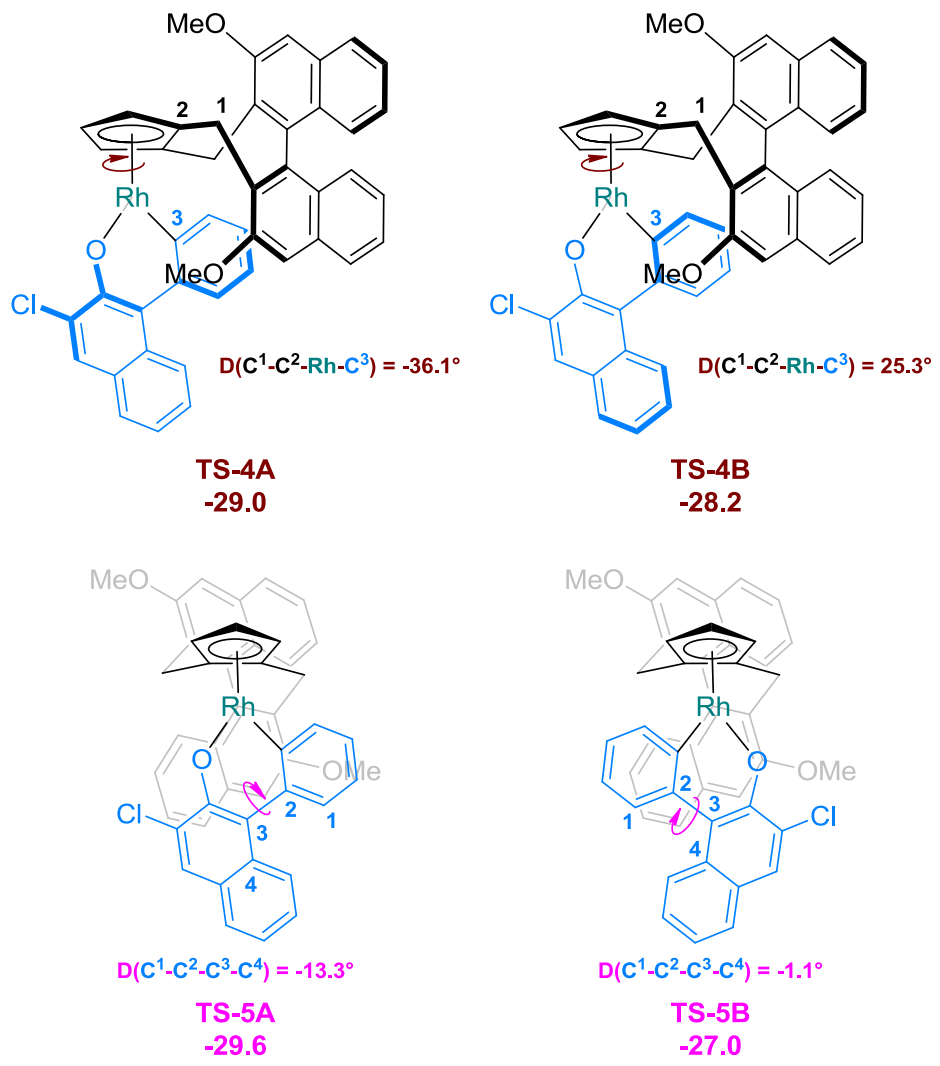

Figure S3. The structures and the relative energies of the four transition states for the interconversion of diastereomers of INT-5. The $\Delta G$ values are in $\mathrm{kcal} / \mathrm{mol}$ and the dihedral angle are in degree. 
4. The intrinsic reaction coordinate (IRC) analyses of TS-3 and TS-6- $\left(S_{\mathrm{a}}, R_{\mathrm{Rh}}\right)$

Intrinsic reaction coordinate (IRC) analyses were conducted for the transition states of turnover-limiting step (TS-3) and selectivity-determining step (TS-6- $\left.\left(S_{\mathrm{a}}, R_{\mathrm{Rh}}\right)\right)$ to confirm the connectivity of these transition states to right intermediates. The results are shown in Figure S4.
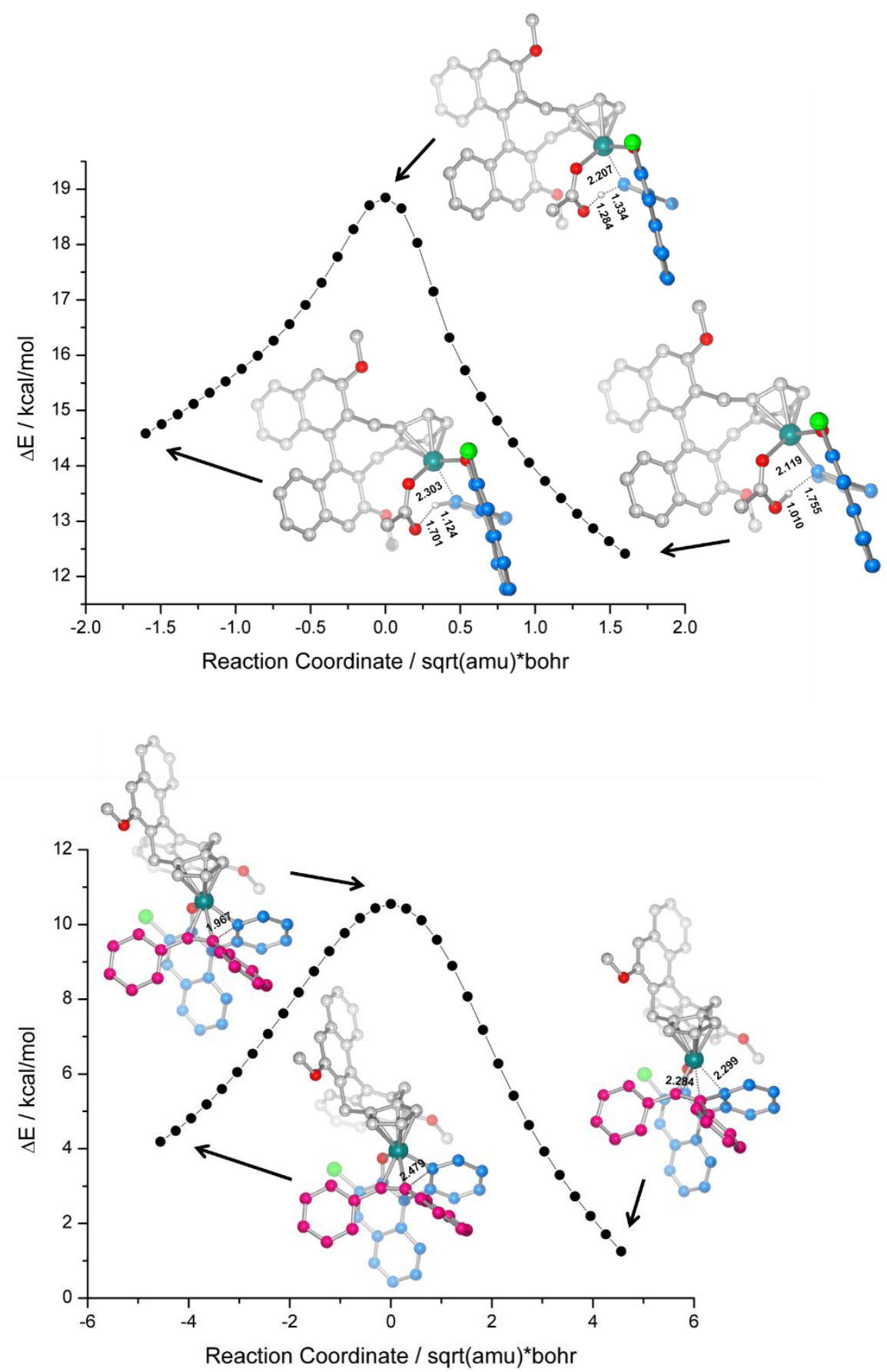

Figure S4. The results of the IRC analyses of TS-3 (up) and TS-6- $\left(S_{\mathrm{a}}, R_{\mathrm{Rh}}\right)$ (bottom). 
5. The structures of the transition states for the migratory insertion with Rh-catalyst $\mathbf{K} \mathbf{2}$

The structures and the relative energies of the transition states for the migratory insertion with $\mathrm{Rh}$ catalyst K2 (TS-6- $\left(S_{\mathrm{a}}, R_{\mathrm{Rh}}\right)-\mathbf{K} 2,0.0 \mathrm{kcal} / \mathrm{mol}$ and TS-6- $\left.\left(R_{\mathrm{a}}, S_{\mathrm{Rh}}\right)-\mathbf{K} 2,2.1 \mathrm{kcal} / \mathrm{mol}\right)$ are shown in Figure S5. The energy gap between these two transition states is smaller than that between TS-6- $\left(S_{\mathrm{a}}, R_{\mathrm{Rh}}\right)$ and TS-6- $\left(R_{\mathrm{a}}, S_{\mathrm{Rh}}\right)$, which is consistent with the experimental trend qualitatively.

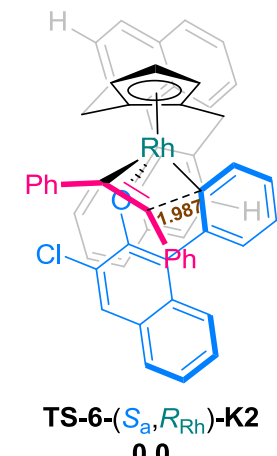

0.0

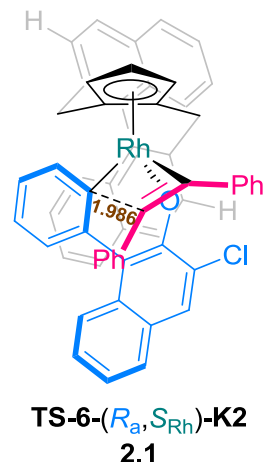

2.1

Figure S5. The structures and the relative energies of the transition states for the migratory insertion with Rh-catalyst $\mathbf{K 2}$. The $\Delta G$ values are in $\mathrm{kcal} / \mathrm{mol}$ and the bond distances are in angstrom. 
6. The reduced density gradient (RDG) analyses of TS-6'-A and TS-7'-A

As suggested by two referees, reduced density gradient (RDG) analyses were conducted to support the existence of $\pi-\pi$ interaction in TS-6' $-\mathbf{A}$ and TS-7'-A. The results are shown in Figure S6.

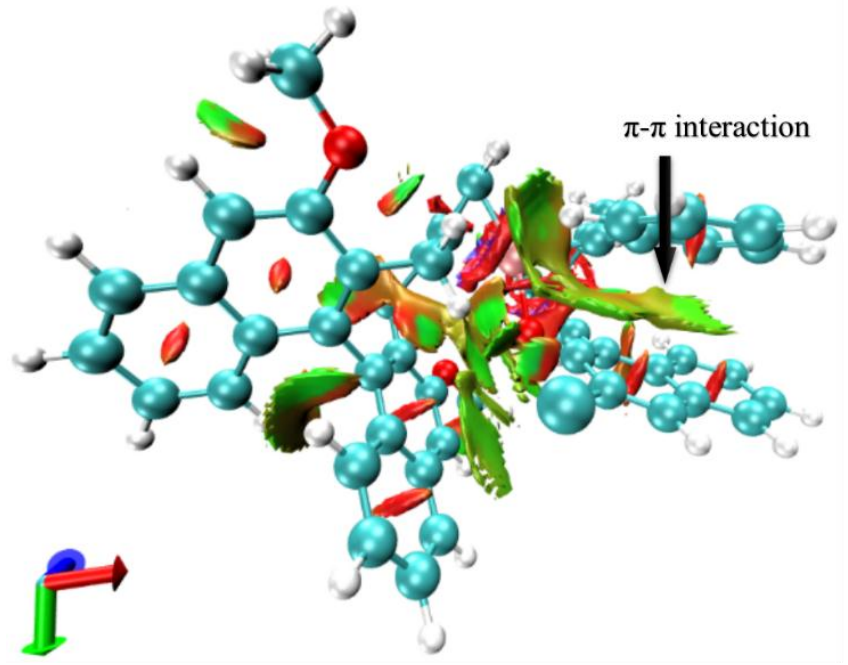

TS-6'-A

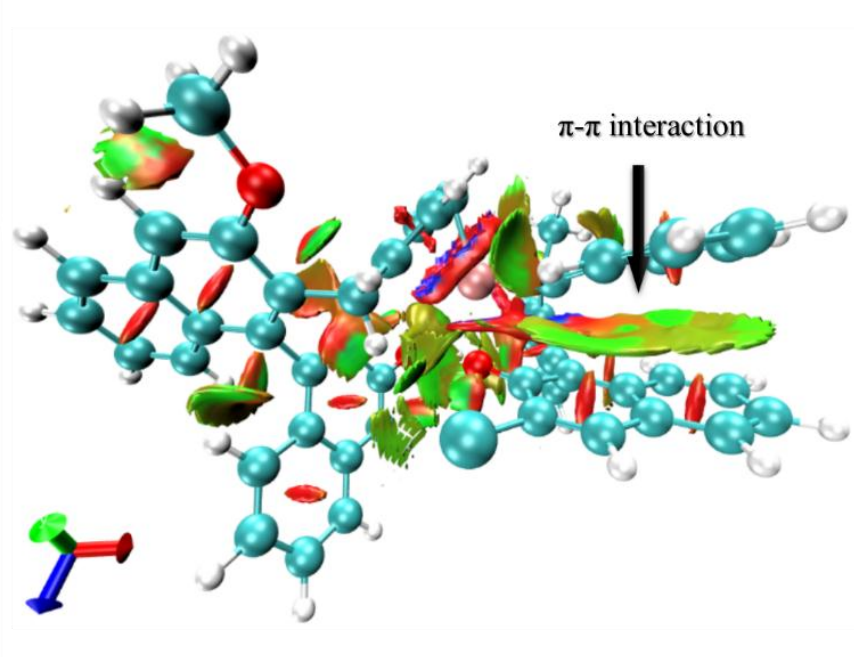

TS-7'-A

Figure S6. The RDG analyses of TS-6'-A and TS-7'-A. 


\section{Cartesian coordinates and energies of all the optimized structures}

\section{HOAC}

Opt \& wB97XD/6-31G (d,p)/5d in toluene (SMD model)

$\mathrm{SCF}$ Done: $\mathrm{E}(\mathrm{RwB} 97 \mathrm{XD})=-229.022707094 \mathrm{a} \cdot \mathrm{u}$.

Zero-point correction $=0.062760$ Hartree/Particle

Sum of electronic and thermal Free Energies = -228.9941 a.u. @ $358.15 \mathrm{~K}$

$\mathrm{SP}$ a B3LYP-D3 (BJ)/def2-TZVPP in toluene (SMD model)

SCF Done: E (RB3LYP) $=-229.205295848$ a.u.

$0,0,6.2793786687,7.2211751413,0.3335458631$

$0,0,6.4100462813,7.3814573521,2.5618925734$

C, $0,6.8288741811,7.6863899993,1.4697179946$

$\mathrm{C}, 0,7.9880026988,8.5999577245,1.1948446314$

$\mathrm{H}, 0,8.4052617473,8.955454876,2.1358407484$

$\mathrm{H}, 0,8.7549566868,8.0679018731,0.6252872368$

$\mathrm{H}, 0,7.6580860435,9.4489977777,0.5898822281$

$\mathrm{H}, 0,5.5463896925,6.645706896,0.5965101642$

\section{$\mathrm{K}_{2} \mathrm{CO}_{3}$}

Opt a wB97XD/6-31G(d,p)/5d in toluene (SMD model)

$\mathrm{SCF}$ Done: $\mathrm{E}(\mathrm{RwB} 97 \mathrm{XD})=-1463.61085960 \mathrm{a} \cdot \mathrm{u}$.

Zero-point correction $=0.016975$ Hartree/Particle

Sum of electronic and thermal Free Energies = -1463.635867 a.u. @ $358.15 \mathrm{~K}$

$\mathrm{Sp}$ a B3LYP-D3 (BJ)/def2-TZVPP in toluene (SMD model)

SCF Done: $E(R B 3 L Y P)=-1463.93460562$ a.u.

$C, 0,-0.4818194277,-0.5596130175,-0.0000261668$

$0,0,0.8430484428,-0.6942465639,-0.0001358379$

$0,0,-0.9811007418,0.6190598281,-0.0339007123$

$0,0,-1.2079944825,-1.6137543229,0.0339550659$

$K, 0,0.6937777162,-3.1898652024,-0.0151180556$

$K, 0,1.1988442429,1.7803905186,0.0152442667$

\section{$\mathrm{KHCO}_{3}$}

Opt a wB97XD/6-31G (d,p)/5d in toluene (SMD model)

$\mathrm{SCF}$ Done: $\mathrm{E}(\mathrm{RwB} 97 \mathrm{XD})=-864.288243065 \mathrm{a} \cdot \mathrm{u}$.

Zero-point correction $=0.028583$ Hartree/Particle

Sum of electronic and thermal Free Energies = -864.297343 a.u. @ $358.15 \mathrm{~K}$

$\mathrm{Sp}$ a B3LYP-D3 (BJ)/def2-TZVPP in toluene (SMD model)

$\mathrm{SCF}$ Done: $\mathrm{E}(\mathrm{RB} 3 \mathrm{LYP})=-864.552935924 \mathrm{a} \cdot \mathrm{u}$.

$C, 0,-0.0467894304,0.7777998522,-0.0000151609$

$0,0,0.0366924965,-0.4803881014,-0.0001515908$

$0,0,1.139945439,1.4663827512,0.0001200813$

$0,0,-1.082672677,1.4763362474,0.0000068964$

$\mathrm{K}, 0,-2.5599963309,-0.6571852808,0.0002296287$

$\mathrm{H}, 0,1.8176125129,0.7814430714,0.0000276953$

\section{KOAC}

Opt a wB97XD/6-31G (d,p)/5d in toluene (SMD model)

$\mathrm{SCF}$ Done: $\mathrm{E}(\mathrm{RwB} 97 \mathrm{XD})=-828.373113553 \mathrm{a} \cdot \mathrm{u}$.

Zero-point correction $=0.050684$ Hartree/Particle

Sum of electronic and thermal Free Energies = -828.361719 a.u. @ $358.15 \mathrm{~K}$ 
Sp @ B3LYP-D3 (BJ)/def2-TZVPP in toluene (SMD model)

$\mathrm{SCF}$ Done: $\mathrm{E}(\mathrm{RB} 3 \mathrm{LYP})=-828.608857469 \mathrm{a} \cdot \mathrm{u}$.

$C, 0,-0.0128536909,0.7913986316,-0.0222992416$

$0,0,0.0363978093,-0.4694631181,-0.0024245153$

$0,0,-1.0687220958,1.4825114128,-0.0131855938$

$\mathrm{K}, 0,-2.553507547,-0.6467945847,0.0274921982$

C, $0,1.3187962535,1.5448544697,-0.0154480089$

$\mathrm{H}, 0,2.1029204136,0.9605437865,-0.5029097759$

$\mathrm{H}, 0,1.220773339,2.5208996693,-0.4965526011$

$\mathrm{H}, 0,1.6250233383,1.7130609429,1.0235599584$

\section{$\mathrm{KHCO}_{3} \cdot \mathrm{KOAC}$}

Opt @ wB97XD/6-31G (d,p)/5d in toluene (SMD model)

$\mathrm{SCF}$ Done: $\mathrm{E}(\mathrm{RwB} 97 \mathrm{XD})=-1692.69403362 \mathrm{a} \cdot \mathrm{u}$.

Zero-point correction $=0.080180$ Hartree/Particle

Sum of electronic and thermal Free Energies = -1692.672119 a.u. @ $358.15 \mathrm{~K}$

$\mathrm{Sp}$ \& B3LYP-D3 (BJ)/def2-TZVPP in toluene (SMD model)

$\mathrm{SCF}$ Done: $\mathrm{E}(\mathrm{RB} 3 \mathrm{LYP})=-1693.19030335 \mathrm{a} \cdot \mathrm{u}$.

$\mathrm{C}, 0,1.0584137857,0.1041578556,0.0090669571$

$0,0,0.3981665796,-0.9527112763,-0.0295776396$

$0,0,2.4290376949,-0.0005815979,0.0971982221$

$0,0,0.6337493053,1.2875042173,-0.0247722023$

$\mathrm{K}, 0,-1.8101420704,0.4614744689,-0.2007308643$

$\mathrm{H}, 0,2.6327449682,-0.9428409083,0.1120755239$

C, $0,3.7683107153,5.6019417373,-0.1399100708$

$0,0,4.654761352,4.7059853006,-0.1546840081$

$0,0,2.5231992795,5.4089989812,-0.076568588$

$\mathrm{K}, 0,2.8382331235,2.8101412133,0.0133895723$

$\mathrm{C}, 0,4.2365171037,7.0617854876,-0.1649846179$

$\mathrm{H}, 0,5.2726564275,7.1442844186,-0.5008465394$

$\mathrm{H}, 0,3.5851399554,7.661929712,-0.8066987073$

$\mathrm{H}, 0,4.1657519798,7.4754743704,0.8477696222$

1

Opt a wB97XD/6-31G (d,p)/5d in toluene (SMD model)

$\mathrm{SCF}$ Done: $\mathrm{E}(\mathrm{RwB} 97 \mathrm{XD})=-1151.53614061 \mathrm{a} \cdot \mathrm{u}$.

Zero-point correction $=0.225961$ Hartree/Particle

Sum of electronic and thermal Free Energies = -1151.363666 a.u. @ $358.15 \mathrm{~K}$

$\mathrm{Sp}$ a B3LYP-D3 (BJ)/def2-TZVPP in toluene (SMD model)

$\mathrm{SCF}$ Done: E(RB3LYP) =-1152.14214429 a.u.

$\mathrm{H}, 0,-1.7471139476,-0.6949447274,-1.3208092511$

$0,0,-2.3024611598,0.0699586002,-1.1271474502$

C, $0,-3.5611851291,-0.3406695589,-0.8705626205$

$\mathrm{C}, 0,-3.9179136631,-1.7166779727,-0.9164147332$

$\mathrm{C}, 0,-4.5206009597,0.5995281034,-0.5395808654$

C, $0,-5.1829556722,-2.1467098009,-0.640337826$

$\mathrm{C}, 0,-5.8572058246,0.1777306805,-0.2586765941$

C, $0,-6.1865713189,-1.2070854856,-0.3042849737$

$\mathrm{C}, 0,-8.4847035001,-0.7136550702,0.2898782448$

$\mathrm{C}, 0,-8.1681819451,0.6643919578,0.3212909811$

$\mathrm{C}, 0,-6.8953570479,1.099196238,0.0547974188$

$C, 0,-7.5128933661,-1.6273299836,-0.0204188156$

$\mathrm{H}, 0,-9.4968635879,-1.0412739123,0.5045926005$ 
$\mathrm{H}, 0,-8.9443110286,1.3868053405,0.5546867837$

$\mathrm{H}, \mathrm{O},-6.6713508326,2.1597928492,0.0752774304$

$\mathrm{H}, \mathrm{O},-7.7418019869,-2.6888407636,-0.0578657789$

$\mathrm{H}, 0,-5.4227195166,-3.2041957962,-0.6781393191$

C, $0,-4.1329375356,2.0377423648,-0.4706045418$

$C, 0,-4.1346483859,2.7143505536,0.7533489357$

C, $0,-3.7629629648,2.7325743655,-1.6256006178$

C, $0,-3.7776754081,4.0579672315,0.8202214511$

$\mathrm{H}, 0,-4.4170490821,2.1812640843,1.6566499084$

C, $0,-3.4039498848,4.0749216242,-1.5588096344$

$\mathrm{H}, \mathrm{O},-3.7559645883,2.2144339863,-2.57957771$

C, $0,-3.4110123498,4.7415103959,-0.3360091313$

$\mathrm{H}, 0,-3.7829249216,4.5693941542,1.7780260191$

$\mathrm{H}, 0,-3.1188091261,4.6012446609,-2.464657615$

$\mathrm{H}, \mathrm{O},-3.131143971,5.7892840988,-0.2844211529$

$\mathrm{Cl}, 0,-2.666401645,-2.8763069583,-1.3300659224$

\section{$2 a$}

Opt a wB97XD/6-31G(d,p)/5d in toluene (SMD model)

$\mathrm{SCF}$ Done: $\mathrm{E}(\mathrm{RwB} 97 \mathrm{XD})=-539.288509469 \mathrm{a} \cdot \mathrm{u}$.

Zero-point correction $=0.193943$ Hartree/Particle

Sum of electronic and thermal Free Energies = -539.144481 a.u. @ $358.15 \mathrm{~K}$

$\mathrm{Sp}$ a B3LYP-D3 (BJ)/def2-TZVPP in toluene (SMD model)

SCF Done: $\mathrm{E}(\mathrm{RB} 3 \mathrm{LYP})=-539.728683137 \mathrm{a} \cdot \mathrm{u}$.

$C, 0,-0.3415474153,1.1302313199,0.0000022301$

$\mathrm{C}, 0,0.8698807375,1.1300954352,-0.0001453932$

$\mathrm{C}, 0,-1.7726860487,1.1303916893,0.0001829791$

C, $0,-2.4818776497,2.0147540783,-0.826586887$

C, $0,-2.4818656643,0.2461844919,0.8271293283$

$\mathrm{C}, 0,-3.8713030772,2.0113797734,-0.8231880433$

$\mathrm{H}, \mathrm{O},-1.9347735291,2.6992875866,-1.4664762274$

$\mathrm{C}, 0,-3.8712909452,0.2498641331,0.8240773371$

$\mathrm{H}, \mathrm{O},-1.9347522604,-0.4384689996,1.4668822611$

C, $0,-4.5692437326,1.1306981402,0.0005312246$

$\mathrm{H}, 0,-4.4114901021,2.6990867169,-1.4663006645$

$\mathrm{H}, \mathrm{O},-4.4114684365,-0.4377255981,1.4673233689$

$\mathrm{H}, \mathrm{O},-5.6547830319,1.1308158473,0.0006657366$

C, $0,2.3010194143,1.1299241734,-0.0003196337$

C, $0,3.0102130263,2.0141249384,-0.8272606959$

$\mathrm{C}, 0,3.0101970332,0.2455528412,0.8264528719$

C, $0,4.3996383992,2.0104298964,-0.8242007158$

$\mathrm{H}, 0,2.4631110965,2.6987856023,-1.467015775$

C, $0,4.3996223677,0.2489117682,0.8230620053$

$\mathrm{H}, 0,2.4630814005,-0.4389754493,1.4663379529$

$\mathrm{C}, 0,5.0975770983,1.1295868733,-0.0006523175$

$\mathrm{H}, 0,4.9398273288,2.698014364,-1.467442752$

$\mathrm{H}, 0,4.9397979541,-0.4388023947,1.4661765244$

$\mathrm{H}, 0,6.1831163949,1.129456727,-0.0007808077$

\section{$2 b$}

Opt a wB97XD/6-31G (d, p)/5d in toluene (SMD model)

$\mathrm{SCF}$ Done: $\mathrm{E}(\mathrm{RwB} 97 \mathrm{XD})=-347.609799876 \mathrm{a} \cdot \mathrm{u}$.

Zero-point correction $=0.139630$ Hartree/Particle

Sum of electronic and thermal Free Energies = -347.513443 a.u. a $358.15 \mathrm{~K}$

$\mathrm{Sp}$ a B3LYP-D3 (BJ)/def2-TZVPP in toluene (SMD model) 
SCF Done: $\mathrm{E}(\mathrm{RB} 3 \mathrm{LYP})=-347.894444919 \mathrm{a} \cdot \mathrm{u}$.

$C, 0,-0.3499204054,1.1329566915,-0.0019042708$

$\mathrm{C}, 0,0.8593082262,1.1315392138,-0.0011026693$

C, $0,-1.7845919259,1.1322365392,-0.0011904414$

$\mathrm{C}, 0,-2.4966031163,2.0147914298,-0.8269287324$

$\mathrm{C}, 0,-2.4939880386,0.2485595929,0.8256390343$

$\mathrm{C}, 0,-3.8865439755,2.0106402297,-0.8230545619$

$\mathrm{H}, 0,-1.9513805721,2.6999594472,-1.4680191526$

C, $0,-3.883931298,0.2504502726,0.8239039408$

$\mathrm{H}, 0,-1.9466147042,-0.4356883602,1.4658817081$

$\mathrm{C}, 0,-4.5838500738,1.1299854158,0.0009450596$

$\mathrm{H}, \mathrm{O},-4.4272477833,2.6980609026,-1.4662517478$

$\mathrm{H}, \mathrm{O},-4.4225257948,-0.4378458841,1.4679380705$

$\mathrm{H}, 0,-5.669490602,1.1291182988,0.0017569274$

$\mathrm{C}, 0,2.3179903411,1.1286430164,0.0007455825$

$\mathrm{H}, 0,2.7081999909,1.3780738939,0.9924698693$

$\mathrm{H}, 0,2.7072587959,0.1440898475,-0.2769234886$

$\mathrm{H}, \mathrm{0}, 2.7108059257,1.8605916027,-0.7112199677$

\section{3}

Opt a wB97XD/6-31G(d,p)/5d in toluene (SMD model)

$\mathrm{SCF}$ Done: $\mathrm{E}(\mathrm{RwB} 97 \mathrm{XD})=-1689.67703748 \mathrm{a} \cdot \mathrm{u}$.

Zero-point correction $=0.401317$ Hartree/Particle

Sum of electronic and thermal Free Energies = -1689.348018 a.u. @ $358.15 \mathrm{~K}$

$\mathrm{Sp}$ a B3LYP-D3 (BJ)/def2-TZVPP in toluene (SMD model)

$\mathrm{SCF}$ Done: $\mathrm{E}(\mathrm{RB} 3 \mathrm{LYP})=-1690.70285805 \mathrm{a} \cdot \mathrm{u}$.

$0,0,-1.5724164875,-0.2510629085,0.1357773523$

$C, 0,-2.1616953666,0.6847778769,0.6287783016$

C, $0,-3.6376915702,0.9918619166,0.2688228597$

$\mathrm{C}, 0,-1.5068927553,1.6368976881,1.5551518776$

$C, 0,-4.360596968,1.9285664057,1.2188216545$

C, $0,-2.1979121586,2.5540648367,2.2513756846$

C, $0,-3.6414461792,2.7067385635,2.1365331179$

$\mathrm{H}, \mathrm{O},-1.677211305,3.2002430915,2.9529064904$

C, $0,-5.6909623524,3.7617934385,2.8704254088$

C, $0,-6.4055422344,2.9844678874,1.9600155821$

C, $0,-5.7434234407,2.0777456453,1.1403757491$

C, $0,-4.3140590217,3.621856525,2.9565520403$

$\mathrm{H}, 0,-6.2072279457,4.470100624,3.5099467093$

$\mathrm{H}, 0,-7.4838507332,3.0839423863,1.8879120089$

$\mathrm{H}, \mathrm{O},-6.3040822134,1.47569003,0.4322394432$

$\mathrm{H}, \mathrm{O},-3.7451369132,4.2177069956,3.6645371628$

C, $0,-3.5531594757,1.6054286985,-1.1283338025$

$C, 0,-3.0090231068,2.8134153118,-1.5267664829$

C, $0,-4.1037138199,0.7097526171,-2.0496058514$

C, $0,-3.0254839659,3.1275811786,-2.8875712047$

$\mathrm{H}, \mathrm{O},-2.5834201635,3.5014613908,-0.8021165017$

C, $0,-4.1075059471,1.0174569459,-3.406354501$

C, $0,-3.5651865939,2.2361576182,-3.814478729$

$\mathrm{H}, \mathrm{O},-2.6112432887,4.0719448684,-3.2259373175$

$\mathrm{H}, \mathrm{O},-4.5200169976,0.3230750628,-4.1317663624$

$\mathrm{H}, 0,-3.5620937695,2.4933563557,-4.8691761082$

C, $0,-4.3921636084,-0.3099079138,0.0035875943$

C, $0,-4.6079253016,-0.4681540519,-1.3242216326$

$\mathrm{Cl}, 0,0.2095128932,1.4508364093,1.7686891386$ 
C, $0,-4.8315800136,-1.1711431203,1.1172465493$

$C, 0,-6.0355826324,-1.8876691535,1.0393068462$

C, $0,-4.0951049976,-1.2479463941,2.307187518$

C, $0,-6.4687132888,-2.672813036,2.1002438591$

$\mathrm{H}, 0,-6.6433491172,-1.8197037871,0.143970732$

$C, 0,-4.5295170884,-2.0335793543,3.3690391213$

$\mathrm{H}, 0,-3.1652682389,-0.699275055,2.4133418404$

C, $0,-5.7157833546,-2.7538345901,3.2692203797$

$\mathrm{H}, \mathrm{O},-7.4042019943,-3.2172684719,2.0153766055$

$\mathrm{H}, \mathrm{O},-3.9361901859,-2.081025719,4.2769309453$

$\mathrm{H}, \mathrm{O},-6.0558183489,-3.3664933535,4.0983806398$

$C, 0,-5.2114573342,-1.6298585949,-2.0119852178$

C, $0,-4.6692177438,-2.9086590416,-1.8446002886$

C, $0,-6.322313019,-1.464757501,-2.8464522753$

C, $0,-5.2360745324,-4.0018816553,-2.4898126321$

$\mathrm{H}, \mathrm{O},-3.801299728,-3.0350469538,-1.2049381986$

$C, 0,-6.8904751802,-2.5614903596,-3.4885397764$

$\mathrm{H}, 0,-6.7491385908,-0.4745965566,-2.979783133$

C, $0,-6.348954271,-3.8318627736,-3.3111247187$

$\mathrm{H}, \mathrm{O},-4.8068254539,-4.9895608629,-2.3526645617$

$\mathrm{H}, 0,-7.7573682824,-2.4227846633,-4.1274053078$

$\mathrm{H}, \mathrm{O},-6.7909148132,-4.6868374964,-3.8134956089$

$3^{\prime}$

Opt a wB97XD/6-31G $(\mathrm{d}, \mathrm{p}) / 5 \mathrm{~d}$ in toluene (SMD model)

SCF Done: E (RwB97XD) $=-1689.67104331 \mathrm{a} \cdot \mathrm{u}$.

Zero-point correction $=0.402281$ Hartree/Particle

Sum of electronic and thermal Free Energies = -1689.339809 a.u. d $358.15 \mathrm{~K}$

Sp \& B3LYP-D3 (BJ)/def2-TZVPP in toluene (SMD model)

SCF Done: E (RB3LYP) $=-1690.69899981$ a.u.

$0,0,-1.2533332919,-0.4700838953,-0.3396717695$

C, $0,-1.7477744584,0.8077832026,-0.2629245273$

C, $0,-2.9726304351,1.0904715551,-0.8326351897$

$C, 0,-0.9825232537,1.819775306,0.3700183486$

C, $0,-3.5418276186,2.3947406581,-0.6467479177$

C, $0,-1.4667868433,3.0932774433,0.4720546963$

C, $0,-2.7668549913,3.3997852953,-0.0005607014$

$\mathrm{H}, \mathrm{O},-0.8708745043,3.8677094962,0.9433953873$

C, $0,-4.5858147306,4.9885091465,-0.2282541504$

C, $0,-5.3762825276,3.9823354641,-0.8277614939$

C, $0,-4.8705256423,2.7239128885,-1.0328924507$

C, $0,-3.3113407359,4.6987526768,0.1813619476$

$\mathrm{H}, 0,-4.9961611498,5.9821636118,-0.0792620677$

$\mathrm{H}, \mathrm{O},-6.3972632707,4.205309853,-1.121410082$

$\mathrm{H}, \mathrm{O},-5.4995941874,1.960313077,-1.4750546641$

$\mathrm{H}, 0,-2.6995409061,5.4556245625,0.6639719412$

$C, 0,-3.6262819876,0.0453484246,-1.6621849115$

C, $0,-4.1182358011,0.4016791661,-2.9268001097$

C, $0,-3.737447814,-1.2967455581,-1.2441543821$

$C, 0,-4.74385487,-0.5243644272,-3.7481743532$

$\mathrm{H}, \mathrm{O},-3.9919382078,1.4230760553,-3.2713883549$

C, $0,-4.3960474994,-2.2138375563,-2.0762341364$

$\mathrm{C}, 0,-4.8950710248,-1.8393106084,-3.3140877273$

$\mathrm{H}, \mathrm{O},-5.1081087778,-0.2214721373,-4.7246920392$

$\mathrm{H}, \mathrm{O},-4.5002174411,-3.2413792519,-1.742346524$

$\mathrm{H}, \mathrm{O},-5.3877892305,-2.5732619946,-3.9439095602$ 
$C, 0,-1.9849826007,-1.3406671222,0.4759340119$

C, $0,-3.1781495255,-1.7839164593,0.040205788$

$\mathrm{Cl}, 0,0.6000999149,1.4388202966,0.9889747238$

C, $0,-1.303625918,-1.6732919804,1.7474051311$

C, $0,-1.9927160521,-1.6656113888,2.9644271879$

C, $0,0.0629071691,-1.9735225885,1.7387293452$

C, $0,-1.3301106782,-1.9698960788,4.1482325335$

$\mathrm{H}, \mathrm{O},-3.0485435351,-1.4166024661,2.9811484325$

C, $0,0.7214738982,-2.2832130882,2.9228913664$

$\mathrm{H}, 0,0.6061139036,-1.9567616775,0.7995722393$

C, $0,0.0264663794,-2.2838146446,4.1303463586$

$\mathrm{H}, 0,-1.8746324511,-1.9552965986,5.0872319098$

$\mathrm{H}, 0,1.7805238066,-2.5211593206,2.9038321907$

$\mathrm{H}, 0,0.5425975184,-2.5215676001,5.0555285143$

C, $0,-3.9590062137,-2.8114910319,0.7846664415$

C, $0,-3.3957891317,-4.0461288545,1.1219708775$

C, $0,-5.2898522687,-2.5572261271,1.1351923425$

C, $0,-4.1400896008,-4.9970903108,1.8126934759$

$\mathrm{H}, \mathrm{O},-2.3675841977,-4.2555228574,0.8440319466$

C, $0,-6.0320978119,-3.5062650663,1.829968177$

$\mathrm{H}, \mathrm{O},-5.7408020235,-1.6073057205,0.8626147882$

C, $0,-5.4581803417,-4.7284691107,2.1726666938$

$\mathrm{H}, \mathrm{O},-3.6889327772,-5.9507858696,2.068958215$

$\mathrm{H}, 0,-7.0604168162,-3.2907697185,2.1038817744$

$\mathrm{H}, \mathrm{O},-6.0380584453,-5.4706820692,2.7126733269$

\section{Cat}

Opt a wB97XD/SDD/6-31G $(\mathrm{d}, \mathrm{p})$ in toluene (SMD model)

$\mathrm{SCF}$ Done: $\mathrm{E}(\mathrm{RwB} 97 \mathrm{XD})=-1836.51351553 \mathrm{a} \cdot \mathrm{u}$.

Zero-point correction $=0.552352$ Hartree/Particle

Sum of electronic and thermal Free Energies $=-1836.052613 \mathrm{a} . \mathrm{u}$. d $358.15 \mathrm{~K}$

Sp \& B3LYP-D3 (BJ)/def2-TZVPP in toluene (SMD model)

SCF Done: E (RB3LYP) $=-1837.90549641 \mathrm{a} \cdot \mathrm{u}$.

$0,0,-3.5167854899,-0.7305107238,2.0403011276$

$0,0,-2.1607319176,0.8545914187,1.4146858827$

$C, 0,-3.0749526878,0.4492602613,2.1938775726$

$C, 0,-3.6502538626,1.3543006053,3.238502752$

$\mathrm{H}, 0,-4.4790123024,1.9110758355,2.789573856$

$\mathrm{H}, 0,-4.0383686986,0.7761097691,4.0783331449$

$\mathrm{H}, 0,-2.8995198384,2.0685747053,3.5802274723$

$\mathrm{Rh}, 0,-2.3385783224,-0.9565951123,0.246820737$

$0,0,-3.8999940377,0.1003788524,-0.5592828026$

$0,0,-4.3794413333,-1.3270643471,-2.2406340103$

C, $0,-4.5737178268,-0.3012905307,-1.591820895$

C $, 0,-5.7281347393,0.6235054443,-1.9472820586$

$\mathrm{H}, \mathrm{O},-6.6467286141,0.2113349685,-1.51661324$

$\mathrm{H}, 0,-5.5851687947,1.6285972081,-1.5471762285$

$\mathrm{H}, 0,-5.8567852487,0.6601660373,-3.0312440386$

C, $0,-1.6635512465,-2.1008521482,-1.4565743263$

C, $0,-2.1083894377,-2.9659212544,-0.4158629443$

C, $0,-1.2435496808,-2.7519974902,0.7168217862$

C, $0,-0.3172317433,-1.7298364538,0.4006384702$

C, $0,-0.5861318729,-1.3074505368,-0.9685983038$

C, $0,0.727699242,-1.1587389599,1.3229650928$

C, $0,0.1903670909,-0.3077229552,-1.7811350756$

$\mathrm{H}, \mathrm{O},-2.1524118638,-1.9788798833,-2.4117701117$ 
$\mathrm{H}, \mathrm{O},-2.9294059554,-3.6656245619,-0.4756941348$

$\mathrm{H}, 0,-1.3271169109,-3.238603336,1.6797248679$

$0,0,-1.4936849805,1.8025867359,-1.9428586931$

$0,0,2.3774086381,-3.319279015,1.5060815431$

C, $0,0.4958008794,0.971898002,-1.0376967762$

C, $0,-0.4397017283,2.0470300246,-1.1331825495$

$C, 0,-0.2381748102,3.2139482845,-0.4418687659$

C, $0,0.9011779199,3.364044086,0.3894802038$

C, 0,1.1221336413,4.5578012483,1.1251939216

C, 0,2.2151399543,4.6894342098,1.9432467361

C, 0,3.1420891294,3.6303139397,2.0665581976

C, $0,2.9565593564,2.4651970094,1.3653233752$

C, $0,1.8401732267,2.3034286199,0.5038174989$

C, $0,1.6063292598,1.098459725,-0.2345542714$

C, $0,-2.5929897656,2.6896888828,-1.8939941548$

C, $0,2.0925554298,-1.1498227612,0.6715063074$

C, $0,2.5208141601,-0.0728676236,-0.0728567259$

C, $0,3.7992394109,-0.0943998773,-0.7191213041$

C, $0,4.2653075762,0.9835975336,-1.5159850806$

C, $0,5.4949246917,0.9290115075,-2.1231164133$

C, $0,6.3165015544,-0.2091131167,-1.9639912288$

C, $0,5.8883481504,-1.2698329673,-1.2065742032$

C, $0,4.6203568848,-1.2439164728,-0.5692937087$

C, $0,4.1588225326,-2.3477583174,0.1958667597$

C, $0,2.921742155,-2.3071495533,0.787206631$

$\mathrm{C}, 0,3.1183832341,-4.5157300968,1.6338678478$

$\mathrm{H}, \mathrm{O},-0.9502351994,4.0291848175,-0.4964575419$

$\mathrm{H}, 0,0.4025574107,5.3668343406,1.0325473215$

$\mathrm{H}, 0,2.3693722965,5.6079910414,2.5014119385$

$\mathrm{H}, \mathrm{O}, 4.0021462476,3.7393951834,2.7197886619$

$\mathrm{H}, 0,3.670789914,1.6542452896,1.4640457134$

$\mathrm{H}, \mathrm{0},-3.0031492121,2.7421074081,-0.8807957399$

$\mathrm{H}, \mathrm{O},-3.3425770007,2.2760836496,-2.5683252711$

$\mathrm{H}, \mathrm{O},-2.3147884389,3.6913118401,-2.2457045618$

$\mathrm{H}, 0,3.6356435561,1.8584560227,-1.6396007475$

$\mathrm{H}, \mathrm{O}, 5.8381764997,1.7618467056,-2.7285972151$

$\mathrm{H}, 0,7.2883429902,-0.2431665891,-2.4468806548$

$\mathrm{H}, 0,6.5152345107,-2.1493545208,-1.0869152919$

$\mathrm{H}, 0,4.7989871471,-3.2181709644,0.2854237044$

$\mathrm{H}, \mathrm{O}, 3.3127336249,-4.9723584747,0.6557234479$

$\mathrm{H}, 0,2.502363753,-5.1912182924,2.2285394558$

$\mathrm{H}, \mathrm{O}, 4.0694097802,-4.3480061915,2.1536038837$

$\mathrm{H}, 0,0.747141932,-1.7536737214,2.2372094975$

$\mathrm{H}, 0,0.4406667233,-0.1401614314,1.5946335836$

$\mathrm{H}, 0,1.1231317981,-0.7895971923,-2.0918684752$

$\mathrm{H}, \mathrm{O},-0.3811314794,-0.0858483309,-2.6830925933$

\section{INT-0}

Opt a wB97XD/SDD/6-31G $(\mathrm{d}, \mathrm{p})$ in toluene (SMD model)

SCF Done: $\mathrm{E}(\mathrm{RwB} 97 \mathrm{XD})=-2988.08019137 \mathrm{a} \cdot \mathrm{u}$.

Zero-point correction $=0.780116$ Hartree/Particle

Sum of electronic and thermal Free Energies $=-2987.412757 \mathrm{a} \cdot \mathrm{u}$. d $358.15 \mathrm{~K}$

Sp a B3LYP-D3 (BJ)/def2-TZVPP in toluene (SMD model)

$\mathrm{SCF}$ Done: $\mathrm{E}(\mathrm{RB} 3 \mathrm{LYP})=-2990.07119749 \mathrm{a} \cdot \mathrm{u}$.

$0,0,0.7040229583,-1.0035101224,2.8319934216$

$0,0,-0.1682541841,-1.464412972,0.8215202052$ 
$C, 0,-0.132165801,-1.6191653372,2.0531566811$

$\mathrm{C}, 0,-1.1082545713,-2.5392076373,2.7200879121$

$\mathrm{H}, \mathrm{O},-1.1373429354,-3.4835330676,2.1732952984$

$\mathrm{H}, 0,-0.8420433349,-2.7111574015,3.76262076$

$\mathrm{H}, \mathrm{O},-2.1113398475,-2.104645611,2.6625289583$

$\mathrm{Rh}, 0,0.7388555224,0.2279698831,-0.2251812484$

$\mathrm{H}, 0,1.4451819244,-0.577463415,2.2435885357$

$0,0,1.7834583112,-1.1670291708,-1.2817908436$

$0,0,2.7239208974,0.2984341227,-2.7164826821$

C, $0,2.5940309989,-0.8266262356,-2.2365361117$

$\mathrm{C}, 0,3.446728411,-1.9794342149,-2.7385292236$

$\mathrm{H}, 0,4.4798616008,-1.8022293867,-2.4238229677$

$\mathrm{H}, 0,3.116330987,-2.9391083441,-2.338630906$

$\mathrm{H}, 0,3.434043083,-1.9997006781,-3.8315259308$

$0,0,2.312182479,-0.0080395182,1.2135349626$

$\mathrm{C}, 0,3.5391982328,-0.4037975565,0.8749236956$

$\mathrm{C}, 0,3.9133485379,-1.7878692868,0.8998968837$

C, 0,4.511062663,0.5189714747,0.4877043038

C, $0,5.1647275699,-2.216254099,0.5655742266$

$\mathrm{C}, 0,5.84489738,0.097855868,0.1752916484$

$\mathrm{C}, 0,6.1660240261,-1.2876699447,0.1974351735$

$\mathrm{C}, 0,8.4583919217,-0.816255212,-0.4386693391$

C, $0,8.1606821994,0.5651034139,-0.4247191005$

C, $0,6.8974170407,1.0089824586,-0.126499946$

$\mathrm{C}, 0,7.4783565491,-1.7208443543,-0.1255748212$

$\mathrm{H}, 0,9.4608393615,-1.1532760698,-0.6833541339$

$\mathrm{H}, 0,8.9428487706,1.284961984,-0.6464215685$

$\mathrm{H}, 0,6.7001036662,2.0741643587,-0.1086746509$

$\mathrm{H}, 0,7.6915312406,-2.786419753,-0.1112339981$

$\mathrm{H}, 0,5.4000305272,-3.2755290633,0.5880819146$ C, 0,4.1479791416,1.957678156,0.3905840181 C, 0,4.3441937025,2.6550929927,-0.8082929966 $\mathrm{C}, 0,3.6143216069,2.6469740884,1.4846312752$ C, 0,4.0391596148,4.0106342229,-0.9028033714 $\mathrm{H}, 0,4.7140537046,2.1192140031,-1.6759692937$ C, 0,3.2941366481,3.9982353778, 1.387011814 $\mathrm{H}, 0,3.4497527729,2.115322275,2.415567956$ C, $0,3.5101650278,4.6869676964,0.1947313808$ $\mathrm{H}, 0,4.2003207654,4.5333094327,-1.8407990973$ $\mathrm{H}, 0,2.8845002661,4.5175125037,2.2487364513$ $\mathrm{H}, 0,3.2678868801,5.7429256873,0.1216992119$ $\mathrm{C}, 0,0.1946128001,1.5558125798,-1.8270186094$ C, $0,0.9148628466,2.2579357525,-0.8190227308$ C, $0,0.1232631112,2.2168178523,0.384100631$ $\mathrm{C}, 0,-1.0394092014,1.4556563052,0.1317220487$ C, $0,-0.9959094634,1.017480746,-1.2588856083$ $\mathrm{C}, 0,-2.1143049366,1.1238592933,1.1308692956$ $\mathrm{C}, 0,-2.0643959947,0.2807782159,-2.0230644595$ $\mathrm{H}, \mathrm{0}, 0.5562767795,1.3677445936,-2.8274516222$ $\mathrm{H}, 0,1.8654193068,2.7509511312,-0.9475452345$ $\mathrm{H}, 0,0.3989565427,2.6440639099,1.3386245295$ $0,0,-1.0131406643,-2.1831927354,-2.4306179626$ $0,0,-3.1682511118,3.6121709943,1.4861741881$ $\mathrm{C}, 0,-2.6187419908,-0.9198054175,-1.291908965$ C, $0,-2.0049867825,-2.1905666407,-1.5156862814$ $\mathrm{C}, 0,-2.4205933419,-3.3006577415,-0.8263725976$ C, $0,-3.4480863639,-3.194666225,0.1446725007$ $\mathrm{C}, 0,-3.8469741067,-4.3161537088,0.918698749$ 
C, $0,-4.801757091,-4.1944047897,1.8963083978$ $\mathrm{C}, 0,-5.411323527,-2.9438139144,2.1429076181$ $\mathrm{C}, 0,-5.0538104698,-1.8437811285,1.4030682181$ $\mathrm{C}, 0,-4.0672793435,-1.9386139476,0.3866402058$ $C, 0,-3.6349378164,-0.8024407909,-0.371631779$ $\mathrm{C}, 0,-0.1542753516,-3.3060030508,-2.4946575211$ $\mathrm{C}, 0,-3.4879259289,1.4684041776,0.603828555$ $\mathrm{C}, 0,-4.2248886275,0.5456507129,-0.1042330168$ $\mathrm{C}, 0,-5.5187651728,0.8860056732,-0.6159468123$ $\mathrm{C}, 0,-6.3061683519,-0.0293772761,-1.3619948709$ $\mathrm{C}, 0,-7.5434523988,0.3296384567,-1.8347927825$ $\mathrm{C}, 0,-8.0512579672,1.6243329372,-1.5871066001$ $\mathrm{C}, 0,-7.3089082725,2.5333473701,-0.8767254724$ C, $0,-6.0258827941,2.1916200801,-0.3755568205$ $\mathrm{C}, 0,-5.2436427006,3.1334789065,0.3444901775$ $\mathrm{C}, 0,-4.0024174687,2.7838909632,0.8120459181$ $\mathrm{C}, 0,-3.6017162485,4.934696508,1.7318421644$ $\mathrm{H}, 0,-1.9489872232,-4.2643028287,-0.980940424$ $\mathrm{H}, \mathrm{O},-3.3706601035,-5.2747895727,0.7307359549$ $\mathrm{H}, 0,-5.0900429203,-5.059470639,2.4857706109$ $\mathrm{H}, \mathrm{0},-6.1645377602,-2.854144595,2.9193059799$ $\mathrm{H}, 0,-5.5249573981,-0.8849576203,1.5943427627$ $\mathrm{H}, \mathrm{O}, 0.3073566685,-3.4965535969,-1.5210473134$ $\mathrm{H}, 0,0.6219451377,-3.048171303,-3.2143888436$ $\mathrm{H}, \mathrm{O},-0.6892888275,-4.1971223825,-2.8471962128$ $\mathrm{H}, 0,-5.9179685833,-1.0236900409,-1.5557106415$ $\mathrm{H}, \mathrm{O},-8.1346819759,-0.382250643,-2.4019663228$ $\mathrm{H}, \mathrm{O},-9.0314972665,1.8997424968,-1.9639471307$ $\mathrm{H}, 0,-7.6943014897,3.5318605673,-0.6890896604$ $\mathrm{H}, 0,-5.6473991918,4.1270098952,0.5028564168$ $\mathrm{H}, \mathrm{O},-3.785058152,5.4771777782,0.7966276235$ $\mathrm{H}, \mathrm{0},-2.7917339698,5.4229867979,2.274784882$ $\mathrm{H}, \mathrm{O},-4.509378112,4.9524917623,2.347322427$ $\mathrm{H}, \mathrm{O},-1.9120943433,1.6621276802,2.0579678663$ $\mathrm{H}, \mathrm{O},-2.080065553,0.0538269593,1.3429679141$ $\mathrm{H}, 0,-2.8697318364,0.9919482827,-2.2347466437$ $\mathrm{H}, 0,-1.6421667027,-0.0296282302,-2.9792486757$ $\mathrm{Cl}, 0,2.7469299183,-3.0005570383,1.3764986728$

\section{TS-1}

Opt \& $\mathrm{wB} 97 \mathrm{XD} / \mathrm{SDD} / 6-31 \mathrm{G}(\mathrm{d}, \mathrm{p})$ in toluene (SMD model) $\mathrm{SCF}$ Done: $\mathrm{E}(\mathrm{RwB} 97 \mathrm{XD})=-2988.05725412 \mathrm{a} \cdot \mathrm{u}$. Zero-point correction $=0.778809$ Hartree/Particle Sum of electronic and thermal Free Energies = -2987.393217 a.u. @ $358.15 \mathrm{~K}$ Imaginary Freq. $=-204.8076 \mathrm{~cm}^{-1}$

$\mathrm{Sp}$ \& B3LYP-D3 (BJ)/def2-TZVPP in toluene (SMD model) SCF Done: $\mathrm{E}(\mathrm{RB} 3 \mathrm{LYP})=-2990.05058809 \mathrm{a} . \mathrm{u}$.

$0,0,-0.8040596615,-1.3062708246,-2.546436766$ $0,0,0.247882721,-1.9292935942,-0.6741114437$ $\mathrm{C}, 0,0.114323394,-1.9806576855,-1.8913673415$ $\mathrm{C}, 0,1.0247973092,-2.7902299706,-2.7741255035$ $\mathrm{H}, 0,1.4149157671,-3.6420068641,-2.2178195639$ $\mathrm{H}, \mathrm{O}, 0.5054516485,-3.1209751864,-3.6748108375$ $\mathrm{H}, 0,1.8742818554,-2.1674987197,-3.0743750896$ $\mathrm{Rh}, 0,-0.9293769329,-0.1167055219,0.9835810679$ $\mathrm{H}, \mathrm{O},-1.4137486647,-0.8792326832,-1.8543105612$ 
$0,0,-1.4025381308,-2.0280005502,1.8335848678$

$0,0,-3.0411240584,-0.622688136,2.2608178224$

C, $0,-2.5635401707,-1.7781513123,2.2985124956$

C, $0,-3.3605717962,-2.9132583008,2.8929850055$ $\mathrm{H}, \mathrm{O},-4.3141817801,-2.9884570691,2.3635131968$ $\mathrm{H}, \mathrm{O},-2.8268179633,-3.8611165983,2.8184849073$ $\mathrm{H}, \mathrm{O},-3.5827263518,-2.6947846441,3.9414599014$ $0,0,-2.2193540303,-0.2983695374,-0.7037835048$ $C, 0,-3.5432437812,-0.3714458179,-0.6815101844$ C, $0,-4.208009478,-1.6410123445,-0.7077373494$ $C, 0,-4.3342061575,0.7729170431,-0.6673301431$ $C, 0,-5.5669725398,-1.755561304,-0.6642172182$ C, $0,-5.7594192275,0.684705408,-0.6340772417$ C, $0,-6.3799842177,-0.5960797844,-0.6164013822$ C, $0,-8.5778197759,0.4310105268,-0.564553417$ C, $0,-7.9694961005,1.706230186,-0.6034016536$ $\mathrm{C}, 0,-6.6035701625,1.8308187366,-0.6404809028$ C, $0,-7.7945887684,-0.6941212195,-0.5758636457$ $\mathrm{H}, 0,-9.659657846,0.3468136109,-0.5332905298$ $\mathrm{H}, 0,-8.5905577265,2.5970864174,-0.6088133649$ $\mathrm{H}, 0,-6.1556427619,2.817704213,-0.6798135172$ $\mathrm{H}, 0,-8.2467670672,-1.6823345125,-0.5580365891$ $\mathrm{H}, \mathrm{O},-6.0329937177,-2.7358543407,-0.6787236837$ C, $0,-3.6594208052,2.0993972882,-0.6899160057$ C, $0,-3.7298638955,2.9642581103,0.4072273699$ C, $0,-2.9510118984,2.513522732,-1.8235807443$ $\mathrm{C}, 0,-3.1141001519,4.2142789222,0.3714558629$ $\mathrm{H}, 0,-4.2762425856,2.649417566,1.2918468262$ C, $0,-2.3360196482,3.7616393547,-1.8631344847$ $\mathrm{H}, \mathrm{O},-2.8883757885,1.8480910945,-2.6787896542$ C, $0,-2.4159833871,4.6174216683,-0.7645294201$ $\mathrm{H}, 0,-3.183018063,4.8743612495,1.2311682452$ $\mathrm{H}, 0,-1.795055882,4.0672006615,-2.7538013817$ $\mathrm{H}, \mathrm{O},-1.9413606837,5.5935634026,-0.7958276908$ C, $0,0.0198892838,0.8206771615,2.6308657742$ C, $0,-0.8833985105,1.7189534318,1.9608393009$ C, $0,-0.3953062261,1.9248325516,0.6339964514$ $C, 0,0.7586996587,1.1135078765,0.4568349467$ C, 0, 1.0409331395, 0.4473305232,1.7183550839 C, $0,1.6152322968,1.0471939443,-0.7725939241$ C, $0,2.2573523514,-0.3503462918,2.0969684637$ $\mathrm{H}, \mathrm{O},-0.0831482865,0.4541316978,3.6432980303$ $\mathrm{H}, 0,-1.7824182096,2.1458876419,2.381462067$ $\mathrm{H}, \mathrm{O},-0.8621203876,2.5239442174,-0.1340634102$ $0,0,1.6726219981,-2.9862799879,1.9864452053$ $0,0,2.1234800206,3.7141033333,-0.8691967935$ C, $0,2.8793563871,-1.2253154804,1.0334457951$ $C, 0,2.5535086459,-2.6152462447,1.0362510834$ C, $0,3.1173136058,-3.4699009646,0.1244258782$ C, $0,3.9888106492,-2.9728842154,-0.8762545359$ C, $0,4.534914335,-3.8309195037,-1.8671841985$ $\mathrm{C}, 0,5.3316832692,-3.3355586963,-2.8674709749$ C, $0,5.6222063266,-1.9540138121,-2.9246163029$ C, 0,5.114618469,-1.1015856901,-1.9761339595 C, $0,4.2910059909,-1.5851025168,-0.9251618586$ C, $0,3.7288679863,-0.7232892426,0.0733724256$ C, $0,0.9650023427,-4.1972597079,1.7957444806$ C, $0,2.9856042859,1.5849084893,-0.4259219206$ 
C, $0,3.9892078226,0.7478094752,0.0094857375$

C, $0,5.2598052291,1.281718996,0.4020837627$

C, $0,6.3130502127,0.459571334,0.8806754285$

C, $0,7.5198682823,1.0026653318,1.2439777572$

C, 0,7.7299815757,2.3963009541,1.1486272778

C, $0,6.7266337378,3.2170300201,0.6994186134$

C, $0,5.4669992889,2.6851164996,0.3191028596$

C, $0,4.4161953859,3.5313250822,-0.1254809044$

C, 0, 3.2019603534,2.995949571,-0.4713143214

C, $0,2.2494377032,5.1195194322,-0.9442365677$

$\mathrm{H}, 0,2.874163877,-4.5266368291,0.126041546$

$\mathrm{H}, 0,4.2992240742,-4.890999884,-1.8245943179$

$\mathrm{H}, 0,5.7369069313,-4.0033983628,-3.62159059$

$\mathrm{H}, 0,6.247952616,-1.5663960585,-3.7223014295$

$\mathrm{H}, 0,5.3409657456,-0.0419157582,-2.02695726$

$\mathrm{H}, \mathrm{O}, \mathrm{O} .5195121428,-4.2243593814,0.7968963244$

$\mathrm{H}, 0,0.1698395062,-4.1993197257,2.5394831219$

$\mathrm{H}, 0,1.6143873669,-5.0686602356,1.9498925897$

$\mathrm{H}, 0,6.1534886082,-0.6109192399,0.9554012577$

$\mathrm{H}, 0,8.3162544727,0.3605020877,1.6064705471$

$\mathrm{H}, 0,8.6884267371,2.8172407861,1.4366642387$

$\mathrm{H}, 0,6.8828500147,4.2901829188,0.6312484047$

$\mathrm{H}, \mathrm{O}, 4.5955561373,4.599603678,-0.1687724987$

$\mathrm{H}, \mathrm{O}, 2.4941042399,5.5532883607,0.032990909$

$\mathrm{H}, 0,1.2772168458,5.492625196,-1.2683025755$

$\mathrm{H}, 0,3.011153613,5.4156444391,-1.6753980261$

$\mathrm{H}, 0,1.1482677727,1.6308857449,-1.5664031596$

$\mathrm{H}, 0,1.6936941928,0.0106995165,-1.0988674221$

$\mathrm{H}, 0,2.9966733336,0.390856956,2.425773184$

$\mathrm{H}, \mathrm{O}, 2.0024089109,-0.9582949646,2.9657180141$

$\mathrm{Cl}, 0,-3.2376804267,-3.0919088709,-0.8151875179$

\section{INT-1}

Opt a wB97XD/SDD/6-31G $(\mathrm{d}, \mathrm{p})$ in toluene (SMD model)

SCF Done: E (RwB97XD) $=-2988.07072243 \mathrm{a} \cdot \mathrm{u}$.

Zero-point correction $=0.780464$ Hartree/Particle

Sum of electronic and thermal Free Energies $=-2987.404803 \mathrm{a} . \mathrm{u}$. \& $358.15 \mathrm{~K}$

Sp a B3LYP-D3 (BJ)/def2-TZVPP in toluene (SMD model)

SCF Done: E (RB3LYP) $=-2990.06341107$ a.u.

$0,0,-0.5384322397,-0.7837759611,-2.62346758$

$0,0,0.6432919458,-2.2275763044,-1.3850689455$

$C, 0,0.4301845429,-1.673526775,-2.4448500555$

C, $0,1.2715823665,-1.8820971825,-3.6767448577$

$\mathrm{H}, \mathrm{O}, 1.9516972488,-2.7190548352,-3.5189188214$

$\mathrm{H}, 0,0.64165072,-2.0564201731,-4.5526881993$

$\mathrm{H}, 0,1.857976534,-0.9769230705,-3.8645649157$

$\mathrm{Rh}, 0,-1.0468975762,0.2737429061,1.3493368831$

$\mathrm{H}, \mathrm{O},-1.0338863499,-0.6707796442,-1.7594121488$

$0,0,-1.3591962811,-1.7553279073,2.0288377183$

$0,0,-2.8968622214,-0.245251097,2.3028071286$

C, $0,-2.5344236389,-1.4646649688,2.3874694191$

C, $0,-3.4935394283,-2.5058747188,2.8716261693$

$\mathrm{H}, 0,-4.3126166609,-2.5875945131,2.151298256$

$\mathrm{H}, \mathrm{O},-2.9978289415,-3.4716568574,2.9698536816$

$\mathrm{H}, \mathrm{O},-3.9206293164,-2.1992755473,3.8298492021$

$0,0,-1.9275749026,-0.3083284634,-0.4640051874$ 
$C, 0,-3.2330051487,-0.5034610251,-0.6092131991$ $\mathrm{C}, 0,-3.750187026,-1.8359549819,-0.7401648594$ $\mathrm{C}, 0,-4.1402301271,0.5487997615,-0.678664185$ $\mathrm{C}, 0,-5.0859247768,-2.0918225129,-0.8699429325$ $\mathrm{C}, 0,-5.5406458154,0.311030023,-0.8227732378$ $\mathrm{C}, 0,-6.0189291402,-1.0269726714,-0.9032302085$ $\mathrm{C}, 0,-8.3022623325,-0.2331022974,-1.1062812101$ $\mathrm{C}, 0,-7.8327123634,1.0991199995,-1.0466923437$ $\mathrm{C}, 0,-6.4936037679,1.3648694488,-0.9116119525$ C, $0,-7.4100206025,-1.2715153113,-1.0393726887$ $\mathrm{H}, 0,-9.3648960496,-0.4289468421,-1.210739649$ $\mathrm{H}, 0,-8.5405291078,1.9201775534,-1.1116497345$ $\mathrm{H}, \mathrm{O},-6.1499958885,2.3929151097,-0.873329352$ $\mathrm{H}, \mathrm{O},-7.7546655944,-2.3008367318,-1.0942448557$ $\mathrm{H}, \mathrm{O},-5.4372311181,-3.1147861809,-0.9633474688$ C, $0,-3.6320978251,1.9470375723,-0.6083468116$ $\mathrm{C}, 0,-3.8298267878,2.7251285326,0.5368306091$ $\mathrm{C}, 0,-2.9842309746,2.5203438336,-1.707072289$ $\mathrm{C}, 0,-3.4013207215,4.0504152399,0.5783773951$ $\mathrm{H}, \mathrm{O},-4.3269991526,2.2817932405,1.3943888684$ $\mathrm{C}, 0,-2.5553363969,3.8449460136,-1.668033718$ $\mathrm{H}, \mathrm{O},-2.8243453939,1.9230414788,-2.5993881186$ $\mathrm{C}, 0,-2.7650594896,4.6154266006,-0.5250656964$ $\mathrm{H}, \mathrm{O},-3.5718020126,4.6443627044,1.4718971446$ $\mathrm{H}, \mathrm{O},-2.061506328,4.2768482931,-2.5333850912$ $\mathrm{H}, \mathrm{0},-2.4379556409,5.6504352337,-0.4960438839$ C, $0,0.1207240597,1.2997149147,2.8262413$ $\mathrm{C}, 0,-0.733300713,2.2206403854,2.1397985421$ $\mathrm{C}, 0,-0.3756792207,2.2168532247,0.7646690779$ C, $0,0.7142674281,1.2979813245,0.5850043978$ C, $0,1.0409867125,0.7551525883,1.8702051237$ C, 0,1.4649715854,1.0467056746,-0.6902633607 $\mathrm{C}, 0,2.2112522953,-0.1110506041,2.246560815$ $\mathrm{H}, 0,0.1030837041,1.0693576229,3.8826166214$ $\mathrm{H}, 0,-1.5579331811,2.7688612569,2.574495971$ $\mathrm{H}, 0,-0.8557115861,2.7785433293,-0.0236833586$ $0,0,1.5030089509,-2.7116919231,2.5438921256$ $0,0,2.1965243675,3.6402244937,-1.0874602307$ C, $0,2.672283853,-1.1493375959,1.2480173209$ C, $0,2.2786176396,-2.5068805303,1.454948431$ $\mathrm{C}, 0,2.6954293037,-3.495743543,0.6024794757$ $\mathrm{C}, 0,3.4867065924,-3.1778138045,-0.5280426505$ $\mathrm{C}, 0,3.8830665005,-4.1788505416,-1.4521824476$ C, $0,4.6250177396,-3.8608892908,-2.5617271124$ C, $0,5.0054511824,-2.5213433873,-2.8008670628$ $\mathrm{C}, 0,4.6398855311,-1.5329380241,-1.9213244487$ $\mathrm{C}, 0,3.8792340663,-1.8332766043,-0.7610293804$ $\mathrm{C}, 0,3.4692267439,-0.8263697918,0.1716731948$ C, $0,0.8441785007,-3.9556031081,2.6637655766$ $\mathrm{C}, 0,2.8980157464,1.4865382421,-0.5033503045$ $\mathrm{C}, 0,3.8542423839,0.5989836294,-0.0644773628$ $\mathrm{C}, 0,5.1968057757,1.0394803444,0.1789164072$ $\mathrm{C}, 0,6.2093649049,0.1645425125,0.6513778599$ $\mathrm{C}, 0,7.4857092535,0.6185943892,0.8716132143$ $\mathrm{C}, 0,7.8105761225,1.9719561824,0.6312738035$ $\mathrm{C}, 0,6.8499687199,2.8430976633,0.1829736866$ C, $0,5.5209865594,2.4038186486,-0.0507713192$ C, $0,4.5163444272,3.305533451,-0.4948701428$ 
$C, 0,3.2358470526,2.8601089408,-0.7005831883$ C, $0,2.4357788484,5.0178877577,-1.2868576483$ $\mathrm{H}, 0,2.3934459094,-4.526114758,0.7501559377$ $\mathrm{H}, 0,3.5783504189,-5.2058120939,-1.2701215814$ $\mathrm{H}, \mathrm{O}, 4.9177312995,-4.6378088907,-3.2616351525$ $\mathrm{H}, 0,5.5858194307,-2.2743672439,-3.6843568258$ $\mathrm{H}, 0,4.9317211322,-0.5054635472,-2.112359131$ $\mathrm{H}, 0,0.2545302562,-4.175267235,1.7682769312$ $\mathrm{H}, 0,0.1752134512,-3.8619162951,3.5190214451$ $\mathrm{H}, 0,1.5565370088,-4.7694360005,2.8527032817$ $\mathrm{H}, 0,5.9628498397,-0.8758235822,0.8354247355$ $\mathrm{H}, 0,8.2492372775,-0.0638988983,1.2312949795$ $\mathrm{H}, 0,8.8236951408,2.3216397687,0.8053948367$ $\mathrm{H}, 0,7.0945577463,3.8861922512,0.0009924923$ $\mathrm{H}, \mathrm{O}, 4.7864392684,4.3438750104,-0.6519376541$ $\mathrm{H}, 0,2.7896537107,5.5016942098,-0.3681869513$ $\mathrm{H}, 0,1.4775041476,5.452331352,-1.5743189829$ $\mathrm{H}, 0,3.1621811287,5.1878867994,-2.0909139421$ $\mathrm{H}, 0,0.9843065227,1.5881951298,-1.5048528556$ $\mathrm{H}, 0,1.4363794502,-0.0185982462,-0.914631308$ $\mathrm{H}, 0,3.0324775628,0.5932262386,2.4335784159$ $\mathrm{H}, 0,1.9829669084,-0.5963246958,3.1959064861$ $\mathrm{Cl}, 0,-2.6261356798,-3.1709850721,-0.7595613614$

\section{INT-2}

Opt \& wB97XD/SDD/6-31G(d,p) in toluene (SMD model)

$\mathrm{SCF}$ Done: $\mathrm{E}(\mathrm{RwB} 97 \mathrm{XD})=-2759.02984214 \mathrm{a} \cdot \mathrm{u}$. Zero-point correction $=0.715880$ Hartree/Particle Sum of electronic and thermal Free Energies = -2758.418328 a.u. @ $358.15 \mathrm{~K}$ $\mathrm{Sp}$ a B3LYP-D3 (BJ)/def2-TZVPP in toluene (SMD model) SCF Done: $\mathrm{E}(\mathrm{RB} 3 \mathrm{LYP})=-2760.84329373 \mathrm{a} \cdot \mathrm{u}$.

$\mathrm{Rh}, 0,0.8087781242,-1.0828034758,-0.4572084776$ $0,0,1.5212406189,0.8702431475,0.1221203357$ $0,0,0.6543929319,0.5621190261,-1.8450133915$ C, $0,1.2362444841,1.3338646026,-1.0240358685$ C, $0,1.6105424718,2.7271071118,-1.4192666119$ $\mathrm{H}, 0,2.393492135,2.6712759004,-2.1814988433$ $\mathrm{H}, 0,1.9847616236,3.2880641513,-0.5626788743$ $\mathrm{H}, 0,0.7471956757,3.2340643324,-1.8563496949$ $0,0,2.6806357698,-1.4657380184,-1.2400758498$ $\mathrm{C}, 0,3.6972882494,-0.6504837522,-1.073177137$ C, $0,4.327262607,-0.4558432219,0.1593026016$ C, 0,4.2174842139,0.0724165399,-2.2004270616 $\mathrm{C}, 0,5.4275113335,0.4433306301,0.2921484266$ $\mathrm{C}, 0,5.2596592618,0.9481880659,-2.0982322053$ $\mathrm{C}, 0,5.8881208609,1.1645129694,-0.8473852332$ $\mathrm{H}, 0,5.6173343039,1.4771135947,-2.9763802282$ $\mathrm{C}, 0,7.5990180748,2.2545571401,0.4855897231$ C, $0,7.1629892551,1.5267355489,1.6165264985$ $C, 0,6.1138964252,0.647675166,1.5237966631$ $\mathrm{C}, 0,6.9741279725,2.0686902091,-0.720210526$ $\mathrm{H}, 0,8.4294826337,2.9481840156,0.5732020247$ $\mathrm{H}, 0,7.6686439362,1.6610102428,2.568335966$ $\mathrm{H}, 0,5.8024546715,0.091375806,2.4009772789$ $\mathrm{H}, 0,7.3050437372,2.610204607,-1.6027589952$ C, $0,3.8054328025,-1.1895274629,1.3453291586$ 
$C, 0,3.8961054458,-2.5835515989,1.4206714374$ C, $0,3.2227954269,-0.501050535,2.4145880254$ C, $0,3.4351229691,-3.2686273884,2.5416224342$ $\mathrm{H}, 0,4.3393665617,-3.1282392898,0.5927594613$ C, $0,2.765151811,-1.1820618061,3.5396797457$ $\mathrm{H}, 0,3.1253152815,0.5770457185,2.3490954681$ C, $0,2.873435396,-2.569637765,3.6094027927$ $\mathrm{H}, 0,3.5256376736,-4.3501941421,2.5875733263$ $\mathrm{H}, 0,2.3209876274,-0.6290144148,4.3625277876$ $\mathrm{H}, \mathrm{0}, 2.5246277665,-3.1029200076,4.4888625819$ C, $0,0.1597646839,-2.2839414939,1.1978963311$ $C, 0,0.5241436043,-3.1308013177,0.1190426131$ C, $0,-0.3069276826,-2.8049219989,-0.9934507883$ C, $0,-1.2324348234,-1.77567522,-0.5784632134$ C, $0,-0.9550460508,-1.4596139601,0.7866799456$ C, $0,-2.2365204757,-1.114017306,-1.4855091633$ C, $0,-1.7102763984,-0.5236439644,1.6963841149$ $\mathrm{H}, 0,0.6432643273,-2.2473714154,2.1651783518$ $\mathrm{H}, \mathrm{O}, 1.3579713577,-3.8185133196,0.1110189051$ $\mathrm{H}, \mathrm{O},-0.2636228812,-3.2446658631,-1.9804407802$ $0,0,-0.1041011123,1.5431412932,2.4314470566$ $0,0,-4.0788204307,-3.0995464077,-1.8052735213$ $\mathrm{C}, 0,-1.9118670657,0.8539838673,1.1098880318$ C, $0,-0.9858242527,1.8840424073,1.4671356751$ C, $0,-1.0449027264,3.1150400106,0.8650177842$ C, $0,-2.0434962582,3.3900554377,-0.1055907428$ C, $0,-2.098333891,4.6438755999,-0.7679805478$ C, $0,-3.0691716969,4.9043578869,-1.7019563758$ C, $0,-4.0342015659,3.9223548432,-2.0168072159$ C, $0,-4.0060784425,2.7007083514,-1.3911601198$ C, $0,-3.0125895129,2.4015395743,-0.4236429585$ C, $0,-2.9280879247,1.1246940944,0.22123029$ C, $0,0.9157729934,2.4641511662,2.7545664288$ $C, 0,-3.6038457386,-1.0117587971,-0.8542418994$ C, $0,-3.9430829927,0.0646819954,-0.0676993829$ C, $0,-5.242564158,0.1487987601,0.5336974243$ $C, 0,-5.630098794,1.2329801886,1.3637406261$ C, $0,-6.8828451318,1.2789751179,1.9227000566$ C, $0,-7.8080838972,0.2391762125,1.6825228331$ C, $0,-7.458260829,-0.8261820089,0.892396304$ C, $0,-6.1702923787,-0.9018609456,0.3013835169$ C, $0,-5.7945633748,-2.0132011908,-0.4988258299$ C, $0,-4.5411134288,-2.0721977177,-1.0518325166$ C, $0,-4.9451544809,-4.1908722666,-2.0421660603$ $\mathrm{H}, \mathrm{O},-0.3346800377,3.895650968,1.1120867995$ $\mathrm{H}, \mathrm{O},-1.3535032591,5.39625967,-0.5225135345$ $\mathrm{H}, \mathrm{O},-3.0968053239,5.8669168852,-2.2034507337$ $\mathrm{H}, \mathrm{O},-4.7977091126,4.1347558425,-2.7584201784$ $\mathrm{H}, \mathrm{0},-4.7455086208,1.9461602921,-1.6391300922$ $\mathrm{H}, 0,1.5503379715,2.6612406812,1.8849642481$ $\mathrm{H}, 0,1.5115677585,1.9957364665,3.5387135611$ $\mathrm{H}, 0,0.5006524345,3.4030137842,3.1424460292$ $\mathrm{H}, \mathrm{0},-4.9228202853,2.0335958443,1.5523351387$ $\mathrm{H}, \mathrm{O},-7.1645468442,2.1169022411,2.5524925649$ $\mathrm{H}, 0,-8.7969429974,0.2842137902,2.1284888503$ $\mathrm{H}, 0,-8.1646801098,-1.6314281737,0.709590395$ $\mathrm{H}, \mathrm{O},-6.5161297832,-2.8080287175,-0.6490880059$ $\mathrm{H}, \mathrm{0},-5.2367102423,-4.6814083587,-1.1053849317$ 
$\mathrm{H}, \mathrm{O},-4.3840119235,-4.8961525033,-2.6559167014$

$\mathrm{H}, 0,-5.8452585261,-3.8787260305,-2.5855266111$

$\mathrm{H}, \mathrm{O},-2.2913060898,-1.6848057594,-2.4130346338$

$\mathrm{H}, 0,-1.8717026425,-0.1150507093,-1.7391946588$

$\mathrm{H}, 0,-2.6786072022,-0.9857186895,1.91436953$

$\mathrm{H}, \mathrm{O},-1.1638993967,-0.4481090969,2.6362029388$

$\mathrm{Cl}, 0,3.483283069,-0.1950495771,-3.7653200058$

\section{TS-2}

Opt a wB97XD/SDD/6-31G $(\mathrm{d}, \mathrm{p})$ in toluene (SMD model)

$\mathrm{SCF}$ Done: $\mathrm{E}(\mathrm{RwB} 97 \mathrm{XD})=-2759.01408152 \mathrm{a} \cdot \mathrm{u}$.

Zero-point correction $=0.714721$ Hartree/Particle

Sum of electronic and thermal Free Energies $=-2758.406413 \mathrm{a} \cdot \mathrm{u}$. @ $358.15 \mathrm{~K}$

Imaginary Freq. $=-44.2011 \mathrm{~cm}^{-1}$

Sp a B3LYP-D3 (BJ)/def2-TZVPP in toluene (SMD model)

$\mathrm{SCF}$ Done: $\mathrm{E}(\mathrm{RB} 3 \mathrm{LYP})=-2760.83181734 \mathrm{a} \cdot \mathrm{u}$.

$\mathrm{Rh}, 0.82541,-1.32382,-0.21436$

$0,1.89244,1.70601,0.2186$

$0,0.64571,0.48223,-1.19205$

C, 1.2123,1.58113,-0.79843

C, $0.97398,2.7453,-1.73835$

$\mathrm{H}, 1.56054,2.58987,-2.64944$

$\mathrm{H}, 1.28312,3.68038,-1.26978$

$\mathrm{H},-0.07877,2.80026,-2.02492$

$0,2.56932,-1.67401,-1.16291$

$\mathrm{C}, 3.53643,-0.77464,-1.13516$

C, $4.256,-0.50433,0.0255$

$\mathrm{C}, 3.9126,-0.08717,-2.33358$

C, $5.33456,0.42457,0.02129$

C, 4.93429,0.81716, -2.364

C, 5.6726,1.09813,-1.18659

$\mathrm{H}, 5.18942,1.324,-3.28946$

C, $7.45837,2.286,-0.05196$

C, $7.12302,1.62417,1.15179$

C, 6.08931,0.72318,1.18963

C, $6.74565,2.02551,-1.19424$

$\mathrm{H}, 8.27725,2.99864,-0.0677$

$\mathrm{H}, 7.68747,1.83515,2.05532$

$\mathrm{H}, 5.83814,0.22406,2.11998$

$\mathrm{H}, 6.99147,2.52965,-2.12528$

C, 3.84778,-1.19113, 1.28508

C, $4.47138,-2.37301,1.69731$

C, $2.82158,-0.65645,2.0741$

$\mathrm{C}, 4.07855,-3.00842,2.87214$

$\mathrm{H}, 5.26853,-2.79231,1.09058$

C, $2.43425,-1.29198,3.25474$

$\mathrm{H}, 2.34559,0.26527,1.74702$

C, $3.05981,-2.46827,3.65732$

$\mathrm{H}, 4.57415,-3.9243,3.18032$

$\mathrm{H}, 1.63721,-0.86097,3.85487$

$\mathrm{H}, 2.7635,-2.9605,4.57897$

C, $-0.07534,-2.58979,1.28324$

C, $0.3094,-3.38496,0.17434$

$C,-0.38229,-2.86097,-0.96214$

$\mathrm{C},-1.24799,-1.78276,-0.52637$

C, $-1.05847,-1.61467,0.87779$ 
$C,-2.12614,-0.96355,-1.42688$

$C,-1.78569,-0.683,1.81253$

$\mathrm{H}, 0.33823,-2.66754,2.2804$

$\mathrm{H}, 1.04873,-4.17296,0.17421$

$\mathrm{H},-0.28927,-3.2014,-1.98526$

$0,-0.04908,1.24682,2.65183$

$0,-3.96952,-2.86184,-2.07722$

$C,-1.90652,0.73748,1.30848$

$C,-0.94929,1.70276,1.75028$

C, $-0.98996,2.99142,1.28204$

C, $-1.96964,3.37889,0.33402$

$C_{,}-1.98879,4.69172,-0.20648$

C, $-2.92597,5.05424,-1.13958$

$C,-3.89255,4.12032,-1.57626$

$C,-3.90024,2.84534,-1.06901$

$C,-2.9427,2.44097,-0.10194$

$C,-2.89648,1.11075,0.42559$

C, $0.907,2.16787,3.15079$

$C,-3.53429,-0.89214,-0.88668$

$C,-3.90855,0.10119,-0.01142$

$C,-5.24383,0.14874,0.50913$

$C,-5.67076,1.14832,1.42186$

$C,-6.95942,1.16629,1.89396$

$\mathrm{C},-7.88319,0.1818,1.47869$

$C,-7.49615,-0.80272,0.60529$

$C,-6.17044,-0.84801,0.10094$

$C,-5.75787,-1.87744,-0.78647$

$C,-4.46981,-1.90665,-1.25567$

C, $-4.83591,-3.89866,-2.49191$

$\mathrm{H},-0.25587,3.72382,1.59474$

$\mathrm{H},-1.23768,5.40259,0.12681$

$\mathrm{H},-2.92488,6.05967,-1.54932$

$\mathrm{H},-4.628,4.41321,-2.31886$

$\mathrm{H},-4.64049,2.12995,-1.41168$

$\mathrm{H}, 1.52955,2.55557,2.33985$

$\mathrm{H}, 1.529,1.60869,3.85093$

$\mathrm{H}, 0.41558,2.989,3.68763$

$\mathrm{H},-4.96451,1.90667,1.74286$

$\mathrm{H},-7.2714,1.93948,2.58888$

$\mathrm{H},-8.90115,0.20484,1.85549$

$\mathrm{H},-8.20185,-1.56531,0.28697$

$\mathrm{H},-6.47957,-2.63496,-1.07023$

$\mathrm{H},-5.20915,-4.47542,-1.63685$

$\mathrm{H},-4.24288,-4.55158,-3.1331$

$\mathrm{H},-5.68519,-3.50678,-3.06433$

$\mathrm{H},-2.11971,-1.40701,-2.42297$

$\mathrm{H},-1.69179,0.0373,-1.50643$

$\mathrm{H},-2.78379,-1.10223,1.98055$

$\mathrm{H},-1.26613,-0.68816,2.7713$

$\mathrm{Cl}, 3.02802,-0.43787,-3.80061$

\section{INT-3}

Opt a wB97XD/SDD/6-31G(d,p) in toluene (SMD model)

SCF Done: E (RwB97XD) $=-2759.01541229$ a.u.

Zero-point correction $=0.715762$ Hartree/Particle

Sum of electronic and thermal Free Energies $=-2758.404091 \mathrm{a} \cdot \mathrm{u}$. d $358.15 \mathrm{~K}$

Sp \& B3LYP-D3(BJ)/def2-TZVPP in toluene (SMD model) 
SCF Done: E (RB3LYP) $=-2760.83140090$ a.u.

$\mathrm{Rh}, 0,0.8728738835,1.2086653195,-0.4509602874$

$0,0,1.8685025127,-1.9929650491,0.4055253588$ $0,0,0.6344381266,-0.218795697,1.0474491774$ C, $0,1.1473229829,-1.3962198014,1.2065570863$ C, $0,0.7943710456,-2.0170915209,2.5456106308$ $\mathrm{H}, \mathrm{O}, 1.3773388563,-1.5180547354,3.3264912229$ $\mathrm{H}, 0,1.0362061469,-3.0807766309,2.5465436802$ $\mathrm{H}, 0,-0.263933375,-1.8741438411,2.7752748832$ $0,0,2.4203486052,1.8958195381,0.6922956152$ $\mathrm{C}, 0,3.4396374205,1.1134433604,0.9562775099$ C, $0,4.1626065768,0.4477437123,-0.0335203931$ $\mathrm{C}, 0,3.8943280185,0.9781958996,2.3108963451$ $\mathrm{C}, 0,5.3069833805,-0.3399823929,0.2838065546$ $\mathrm{C}, 0,4.9863478474,0.2330311331,2.6442517021$ C, $0,5.7233962504,-0.447416434,1.6405799399$ $\mathrm{H}, 0,5.2987684037,0.1550548717,3.6810040653$ C, $0,7.5597695838,-1.8949293246,0.9925942481$ $\mathrm{C}, 0,7.14780731,-1.7969299664,-0.3555974799$ $\mathrm{C}, 0,6.0529416724,-1.0450825641,-0.7009477544$ $\mathrm{C}, 0,6.85562665,-1.2351441228,1.9675294473$ $\mathrm{H}, 0,8.4253579929,-2.4961057454,1.2528002083$ $\mathrm{H}, 0,7.7008977277,-2.326835741,-1.1254651449$ $\mathrm{H}, 0,5.7428303673,-0.9902436031,-1.7394545104$ $\mathrm{H}, 0,7.1559590506,-1.306617497,3.0096646854$ C, $0,3.6891013443,0.5714379831,-1.4354678681$ $\mathrm{C}, 0,4.3838394519,1.3575157285,-2.3642825747$ C, $0,2.5332980217,-0.1125582007,-1.8543173477$ C, $0,3.94555303,1.4489619506,-3.6798537807$ $\mathrm{H}, 0,5.2737812797,1.8921027796,-2.0462751704$ C, $0,2.1098081995,-0.023554877,-3.1905171529$ $\mathrm{H}, 0,2.0943741732,-0.8545980605,-1.1758127507$ C, $0,2.8079790974,0.7542238365,-4.1014153772$ $\mathrm{H}, 0,4.4967961263,2.061242688,-4.3876485527$ $\mathrm{H}, \mathrm{O}, 1.225180453,-0.5762436595,-3.4929342895$ $\mathrm{H}, \mathrm{0}, 2.4818021765,0.8186046033,-5.1350921826$ $\mathrm{C}, 0,-0.1650314782,1.9392926201,-2.2172439647$ C, $0,0.3251451736,3.040189951,-1.4567863345$ C, $0,-0.2925100055,2.9559715257,-0.1710416165$ C, $0,-1.1658194147,1.8139275363,-0.1497461928$ $\mathrm{C}, 0,-1.0844388999,1.1703455339,-1.4377939782$ C, $0,-1.9820894697,1.3641701523,1.0254661619$ C, $0,-1.8801460912,-0.0036243873,-1.9475042618$ $\mathrm{H}, \mathrm{O}, \mathrm{O} .1443863334,1.6933231485,-3.2234245072$ $\mathrm{H}, 0,1.0453464866,3.7766484299,-1.7826148235$ $\mathrm{H}, 0,-0.1147808583,3.610613213,0.6718660072$ $0,0,-0.1924292791,-2.1547212672,-2.1216037956$ $0,0,-3.7424571548,3.4432996874,1.1508191025$ C, $0,-1.9898971877,-1.1593191008,-0.9781813966$ C, $0,-1.0746038273,-2.2502311615,-1.0975689518$ C, $0,-1.1262839656,-3.3059838351,-0.2237514855$ $\mathrm{C}, 0,-2.0687473445,-3.3155322428,0.8344581333$ $\mathrm{C}, 0,-2.0973219621,-4.3724102451,1.7820215746$ $\mathrm{C}, 0,-2.9911151223,-4.3595169453,2.8218452112$ $\mathrm{C}, 0,-3.9006682788,-3.2872410671,2.9642658915$ $\mathrm{C}, 0,-3.898646223,-2.2542807216,2.0609624883$ $\mathrm{C}, 0,-2.9870469807,-2.2416426492,0.9724425018$ 
$C, 0,-2.934834968,-1.1699330489,0.0244023354$ $C, 0,0.738220503,-3.2135598565,-2.2893430829$ $C, 0,-3.4287441699,1.1808614043,0.6303426383$ $C, 0,-3.886718821,-0.0250694345,0.1509039058$ C, $0,-5.2541373514,-0.1793905849,-0.2511368986$ $C, 0,-5.7622188949,-1.3991659884,-0.7693662672$ C, $0,-7.0788654195,-1.5117515867,-1.1403106388$ $C, 0,-7.9509654153,-0.4079932463,-1.0127138352$ $C, 0,-7.4857949003,0.7861094946,-0.5230772761$ C, $0,-6.1289195965,0.9337437968,-0.134267951$ C, $0,-5.6350174638,2.1732674364,0.352367985$ C, $0,-4.316682196,2.2975955225,0.7094779174$ C, $0,-4.560477139,4.5882259561,1.2808998283$ $\mathrm{H}, 0,-0.4230712397,-4.126297583,-0.2944527761$ $\mathrm{H}, \mathrm{O},-1.3880435442,-5.1883221933,1.6736328532$ $\mathrm{H}, \mathrm{O},-2.9981227143,-5.1708746046,3.5432679991$ $\mathrm{H}, 0,-4.600263131,-3.2805777534,3.794186422$ $\mathrm{H}, \mathrm{O},-4.5946482998,-1.4301345313,2.177966731$ $\mathrm{H}, \mathrm{O}, 1.3712386359,-3.3129876291,-1.4032720963$ $\mathrm{H}, 0,1.3542580117,-2.9420215048,-3.1476179055$ $\mathrm{H}, 0,0.222486767,-4.1569971685,-2.5068666698$ $\mathrm{H}, 0,-5.0960138171,-2.2497457704,-0.8679290873$ $\mathrm{H}, 0,-7.4529544611,-2.4518241213,-1.5331874092$ $\mathrm{H}, \mathrm{O},-8.9915885487,-0.5067120709,-1.3062899466$ $\mathrm{H}, \mathrm{0},-8.1520878255,1.6393082273,-0.4276570976$ $\mathrm{H}, \mathrm{O},-6.3200852095,3.0104881902,0.4210835969$ $\mathrm{H}, 0,-4.9874005145,4.8894722494,0.3164767868$ $\mathrm{H}, 0,-3.9124062359,5.3837670486,1.6502585625$ $\mathrm{H}, \mathrm{O},-5.3706864603,4.4227808725,2.0012874458$ $\mathrm{H}, \mathrm{O},-1.8894828838,2.1023135621,1.8224365398$ $\mathrm{H}, 0,-1.5550934673,0.4252255561,1.3897105665$ $\mathrm{H}, \mathrm{O},-2.8827652512,0.3592392053,-2.1986922036$ $\mathrm{H}, 0,-1.4193095044,-0.3470567392,-2.8749196232$ $\mathrm{Cl}, 0,3.0086309257,1.8181470493,3.5633180637$

\section{TS-3 or TS-3- $\left(R_{\mathrm{a}}, S_{\mathrm{Rh}}\right)$-Back}

Opt a wB97XD/SDD/6-31G (d,p) in toluene (SMD model)

$\mathrm{SCF}$ Done: $\mathrm{E}(\mathrm{RwB} 97 \mathrm{XD})=-2758.99999224 \mathrm{a} \cdot \mathrm{u}$. Zero-point correction $=0.711365$ Hartree/Particle Sum of electronic and thermal Free Energies $=-2758.389964 \mathrm{a} . \mathrm{u}$. d $358.15 \mathrm{~K}$ Imaginary Freq. $=-1205.5884 \mathrm{~cm}^{-1}$

Sp \& B3LYP-D3 (BJ)/def2-TZVPP in toluene (SMD model)

SCF Done: E (RB3LYP) $=-2760.81420151$ a.u.

$\mathrm{Rh}, 0,2.3394629253,0.0187219263,-0.1274385069$ $0,0,0.2748901356,0.7406825787,2.2160597349$ $0,0,1.3776807367,1.7725278848,0.5794663554$ $\mathrm{C}, 0,0.5840760348,1.7784949014,1.5620274957$ $\mathrm{C}, 0,0.0244280246,3.102374895,1.9993627491$ $\mathrm{H}, 0,0.7843685052,3.5960431828,2.6142249579$ $\mathrm{H}, \mathrm{0},-0.875916644,2.9643722447,2.5978517796$ $\mathrm{H}, 0,-0.1824796457,3.7354299635,1.1355369471$ $0,0,3.9694774543,0.692527469,0.9259475669$ C, $0,3.8734605873,0.9910576139,2.1911185144$ C, 0,3.2983825015,0.14827899,3.1496848741 C, $0,4.443664577,2.230655957,2.6472022179$ C, $0,3.2083623443,0.5555224986,4.5220305415$ 
C, $0,4.420478371,2.6117010172,3.9533879654$ $C, 0,3.7894583935,1.7917012099,4.9257242756$ $\mathrm{H}, 0,4.8657112882,3.5541915156,4.256804047$ C, $0,3.0651044776,1.4416452181,7.2158364405$ C, $0,2.4482875402,0.235999394,6.8180392071$ C, $0,2.514275848,-0.1919768608,5.5148717391$ C, $0,3.7145906193,2.2048192772,6.2793158354$ $\mathrm{H}, 0,3.0103814357,1.7665768987,8.2500724388$ $\mathrm{H}, 0,1.9061395224,-0.3571017146,7.5487653148$ $\mathrm{H}, 0,2.0119326784,-1.110144619,5.233587415$ $\mathrm{H}, 0,4.1772504057,3.1474763398,6.5602313376$ C, $0,2.7676454106,-1.1564739276,2.6979159657$ C, $0,3.1381120545,-2.3342744539,3.3646670643$ C, $0,1.8492872047,-1.2313150734,1.6186690848$ $C, 0,2.5730525576,-3.5563833866,3.0253603373$ $\mathrm{H}, 0,3.8760447363,-2.285914163,4.1597733012$ C, $0,1.2569112371,-2.4789362302,1.3348914406$ $\mathrm{H}, 0,0.9972715558,-0.213312958,1.750688037$ $C, 0,1.602970804,-3.6310679924,2.0240310856$ $\mathrm{H}, 0,2.8773917706,-4.4536274291,3.5567211262$ $\mathrm{H}, 0,0.4887650549,-2.5205837245,0.5684414605$ $\mathrm{H}, 0,1.1292255772,-4.5796286459,1.7886345278$ C, $0,2.1120573525,-1.5869172613,-1.5846032511$ C, $0,3.4417591254,-1.0809891667,-1.6100726722$ C, $0,3.3651387779,0.2921819475,-2.0296413667$ $C, 0,2.0027768737,0.633060639,-2.2130144918$ $\mathrm{C}, 0,1.1989423394,-0.5429089029,-1.9217796738$ C, $0,1.4854175124,1.9980432064,-2.571645291$ C, $0,-0.2834726959,-0.7222024973,-2.1307736601$ $\mathrm{H}, \mathrm{O}, 1.8318792265,-2.5944192597,-1.3107785408$ $\mathrm{H}, 0,4.341493932,-1.6319709826,-1.3762201948$ $\mathrm{H}, 0,4.1956832436,0.9789409648,-2.1188210607$ $0,0,-1.4545005814,-1.0076662793,0.3177031691$ $0,0,2.4483824617,2.2211172163,-5.1136219206$ $C, 0,-1.1330528627,0.3821698463,-1.5453599284$ C, $0,-1.7011041776,0.1979496149,-0.2460061877$ C, $0,-2.445761104,1.1884237552,0.3420203379$ C, $0,-2.643345979,2.4256868193,-0.3230192747$ C, $0,-3.3659283045,3.4825212623,0.2900731264$ C, $0,-3.5368966422,4.6824516427,-0.3523059038$ C, $0,-2.9946661874,4.8815526302,-1.6415837823$ $\mathrm{C}, 0,-2.2941878417,3.8760745188,-2.2594217055$ $C, 0,-2.1019681831,2.6233403038,-1.620516253$ C, $0,-1.3554712541,1.5596146843,-2.2240760623$ C, $0,-2.0372686929,-1.2899427519,1.5780242163$ C, $0,0.5566042599,1.9392099882,-3.7609988252$ C, $0,-0.7944844154,1.7340588596,-3.599040315$ C, $0,-1.662977714,1.651763944,-4.7373845456$ C, $0,-3.0581532946,1.4200489545,-4.6172157594$ C, $0,-3.8594589454,1.3512247879,-5.7294176121$ $C, 0,-3.3033062638,1.5076946256,-7.0182336644$ $C, 0,-1.9567837939,1.724644905,-7.165882944$ C, $0,-1.1031686224,1.8002318649,-6.034692923$ C, $0,0.2942444761,2.0056925022,-6.1818746611$ C, $0,1.1037593932,2.058878772,-5.0760274155$ C, $0,3.0598898568,2.4030487489,-6.3746121011$ $\mathrm{H}, \mathrm{O},-2.8714788503,1.058144868,1.3297807813$ $\mathrm{H}, \mathrm{O},-3.7764241817,3.3255930346,1.2839447249$ 
$\mathrm{H}, \mathrm{O},-4.0874450224,5.4842095568,0.1302191885$

$\mathrm{H}, \mathrm{O},-3.129872524,5.8348982301,-2.1423807603$

$\mathrm{H}, \mathrm{O},-1.8742250442,4.0358126946,-3.2467307945$

$\mathrm{H}, 0,-1.6795819152,-0.5930509564,2.3408833739$

$\mathrm{H}, \mathrm{O},-1.7174357297,-2.2998141521,1.8373649732$

$\mathrm{H}, \mathrm{O},-3.1323050451,-1.2621704567,1.5187177108$

$\mathrm{H}, \mathrm{O},-3.4908443233,1.2989034293,-3.630068949$

$\mathrm{H}, \mathrm{O},-4.9249475018,1.1758436544,-5.6194434023$

$\mathrm{H}, \mathrm{O},-3.9445887019,1.4520651801,-7.8925655059$

$\mathrm{H}, \mathrm{O},-1.523188985,1.8403596771,-8.1555324014$

$\mathrm{H}, 0,0.6987621898,2.1054777128,-7.1826362122$

$\mathrm{H}, 0,2.9141092831,1.530044043,-7.0223742177$

$\mathrm{H}, \mathrm{0}, 4.1252490583,2.5300823496,-6.1800195756$

$\mathrm{H}, 0,2.6767823163,3.2983099495,-6.8793479206$

$\mathrm{H}, \mathrm{O}, 2.3343958557,2.6497035013,-2.7806898117$

$\mathrm{H}, 0,0.9610255986,2.4081851053,-1.7043351445$

$\mathrm{H}, 0,-0.4638522389,-0.7891203332,-3.2092413529$

$\mathrm{H}, 0,-0.5764394782,-1.678385569,-1.6947220363$

$\mathrm{Cl}, 0,5.1933969942,3.2720565213,1.4580972552$

\section{TS-3- $\left(S_{\mathrm{a}}, R_{\mathrm{Rh}}\right)$-Back}

Opt a wB97XD/SDD/6-31G(d,p) in toluene (SMD model)

$\mathrm{SCF}$ Done: $\mathrm{E}(\mathrm{RwB} 97 \mathrm{XD})=-2758.99079434 \mathrm{a} \cdot \mathrm{u}$.

Zero-point correction $=0.711318$ Hartree/Particle

Sum of electronic and thermal Free Energies $=-2758.381666 \mathrm{a} . \mathrm{u}$. @ $358.15 \mathrm{~K}$

Imaginary Freq. $=-1180.8145 \mathrm{~cm}^{-1}$

Sp a B3LYP-D3 (BJ)/def2-TZVPP in toluene (SMD model)

SCF Done: E (RB3LYP) $=-2760.80589711$ a.u.

$\mathrm{Rh}, 0,0.7028772762,-1.5458740838,-0.3326732681$

$0,0,3.7209008601,-2.5643791587,-0.7521614784$

$0,0,2.0162276629,-1.8117117975,-1.9756084459$

$C, 0,3.2021476276,-2.227786329,-1.8544359491$

C, $0,4.0557585522,-2.2707350889,-3.0917352286$

$\mathrm{H}, 0,4.4626050565,-1.2657299209,-3.2458832783$

$\mathrm{H}, 0,4.8874630795,-2.9648852292,-2.9670655571$

$\mathrm{H}, 0,3.4574034315,-2.5331339377,-3.965329635$

$0,0,1.1121649465,0.4624579824,-0.5525923693$

C, $0,2.343684057,0.869592886,-0.6796608335$

C, $0,3.3604712819,0.5315010298,0.2184689044$

C, $0,2.6743277985,1.7540039905,-1.7636934017$

C, $0,4.7066306786,0.9741255596,0.0012015048$

C, $0,3.933922118,2.2313911674,-1.9602864631$

C, $0,4.9896433504,1.8378561663,-1.0960493387$

$\mathrm{H}, 0,4.1445589546,2.8988501825,-2.7902549698$

$\mathrm{C}, 0,7.347748978,1.8745571518,-0.5216706401$

C, $0,7.0841105946,0.9884871806,0.5453669653$

C, $0,5.8077401493,0.5494336486,0.7974351777$

C, $0,6.3155350318,2.2820599198,-1.3275931502$

$\mathrm{H}, 0,8.3605533027,2.2176828736,-0.7083260048$

$\mathrm{H}, 0,7.9022759506,0.6401397705,1.1689873221$

$\mathrm{H}, 0,5.637640665,-0.1489064204,1.6086414915$

$\mathrm{H}, 0,6.4995430184,2.9483831842,-2.1666009234$

$\mathrm{C}, 0,3.0037148504,-0.3234412834,1.3715673037$

C, $0,3.3762012454,0.0628491901,2.6669771888$

C, $0,2.3373378062,-1.5632631167,1.1826731972$

C, $0,3.1803746558,-0.7825906336,3.7526692022$ 
$\mathrm{H}, 0,3.8373576868,1.0343420439,2.818428161$ C, $0,2.2221761425,-2.418782989,2.2910195149$ $\mathrm{H}, 0,2.8878476841,-2.1780775233,0.1469850648$ $\mathrm{C}, 0,2.6344039174,-2.0517324437,3.5630382443$ $\mathrm{H}, 0,3.4823925477,-0.4602896903,4.7450803494$ $\mathrm{H}, \mathrm{O}, 1.8273423258,-3.4146329615,2.1316705257$ $\mathrm{H}, 0,2.5359001739,-2.7461201205,4.3923418714$ $\mathrm{Cl}, 0,1.3915536133,2.2206574129,-2.8583001907$ C, $0,-0.9069921296,-2.5110591903,-1.4219929665$ C, $0,-1.3161355982,-1.2164526858,-1.0302633897$ $\mathrm{C}, 0,-1.3003431622,-1.1875033649,0.4064062894$ $\mathrm{C}, 0,-0.935959217,-2.4777466169,0.8860715472$ $\mathrm{C}, 0,-0.644404872,-3.3092933178,-0.2536771903$ C, $0,-1.0014922366,-2.8898863625,2.3326646096$ $\mathrm{C}, 0,-0.3941705885,-4.7949522566,-0.2996297516$ $\mathrm{H}, 0,-0.7465472679,-2.840949198,-2.4405400525$ $\mathrm{H}, 0,-1.5257682249,-0.3827155974,-1.6850033692$ $\mathrm{H}, 0,-1.5487216787,-0.3406943063,1.031370315$ $0,0,2.2219174841,-5.3235476266,-0.8818618611$ $0,0,-3.6864273308,-2.6713828831,2.7999126163$ $\mathrm{C}, 0,0.5487504846,-5.3016479964,0.7633736915$ C, $0,1.9224845954,-5.5143853314,0.4234089789$ $\mathrm{C}, 0,2.8281336822,-5.9020738385,1.3788745946$ $\mathrm{C}, 0,2.4142702476,-6.0924814138,2.7231322726$ $\mathrm{C}, 0,3.3454832312,-6.4344254326,3.7381230404$ $\mathrm{C}, 0,2.939033414,-6.6006647694,5.0376464091$ $\mathrm{C}, 0,1.5792810695,-6.4355130907,5.3847654977$ $\mathrm{C}, 0,0.6556958004,-6.1074606263,4.4240508477$ $\mathrm{C}, 0,1.0468412679,-5.9259485485,3.0717798215$ C, $0,0.1187423914,-5.5417891221,2.050174129$ $\mathrm{C}, 0,3.5343679704,-5.6283796559,-1.3150042698$ $\mathrm{C}, 0,-1.867565365,-4.1167676725,2.4999132395$ $\mathrm{C}, 0,-1.3318692481,-5.3787494759,2.3795345972$ $C, 0,-2.1596164584,-6.5406204702,2.5226976484$ C, $0,-1.652601094,-7.859616999,2.389539037$ C, $0,-2.4750798515,-8.9483164139,2.5379667157$ $\mathrm{C}, 0,-3.8467872327,-8.7703319939,2.8238898045$ $\mathrm{C}, 0,-4.3668256286,-7.5070799534,2.9488151391$ $\mathrm{C}, 0,-3.541853226,-6.362208173,2.7983961776$ $\mathrm{C}, 0,-4.0786895848,-5.0518477158,2.9046977153$ $C, 0,-3.2661364212,-3.9582959035,2.7456831529$ C, $0,-5.0601076687,-2.4305859751,3.0289864496$ $\mathrm{H}, 0,3.8735210601,-6.048768944,1.1345235269$ $\mathrm{H}, 0,4.3909253984,-6.5551007411,3.467995064$ $\mathrm{H}, 0,3.6631201177,-6.8570758911,5.8048961979$ $\mathrm{H}, 0,1.2662811952,-6.5665070469,6.415791377$ $\mathrm{H}, \mathrm{O},-0.3870786036,-5.9764211714,4.6938829108$ $\mathrm{H}, 0,4.2726634498,-4.9767774803,-0.8388866996$ $\mathrm{H}, 0,3.5421474435,-5.4519941855,-2.3915469122$ $\mathrm{H}, 0,3.7766717212,-6.6811680416,-1.1234362889$ $\mathrm{H}, 0,-0.6000838921,-8.0024247533,2.1693641633$ $\mathrm{H}, \mathrm{O},-2.0711299485,-9.9504688384,2.4348692091$ $\mathrm{H}, 0,-4.489707724,-9.6372709767,2.9416197106$ $\mathrm{H}, \mathrm{O},-5.4226222764,-7.3650546787,3.1632637099$ $\mathrm{H}, \mathrm{0},-5.139114565,-4.9434285943,3.1014483515$ $\mathrm{H}, \mathrm{O},-5.6805862497,-2.8652521098,2.2359551059$ $\mathrm{H}, \mathrm{O},-5.1824407375,-1.3470236034,3.0260744652$ $\mathrm{H}, \mathrm{O},-5.3815556851,-2.8251233566,4.0007125691$ 
$\mathrm{H}, \mathrm{O},-1.4046598113,-2.0573551263,2.9098763491$

$\mathrm{H}, 0,-0.0090312476,-3.10027523,2.7227567505$

$\mathrm{H}, \mathrm{O},-1.3631496038,-5.2971851897,-0.1995911504$

$\mathrm{H}, \mathrm{O},-0.001382276,-5.0427096748,-1.286309789$

\section{TS-3- $\left(R_{\mathrm{a}}, S_{\mathrm{Rh}}\right)$-Front}

Opt a wB97XD/SDD/6-31G $(d, p)$ in toluene (SMD model)

SCF Done: $\mathrm{E}(\mathrm{RwB} 97 \mathrm{XD})=-2758.99309013 \mathrm{a} \cdot \mathrm{u}$.

Zero-point correction $=0.710612$ Hartree/Particle

Sum of electronic and thermal Free Energies $=-2758.385381 \mathrm{a} . u$. @ $358.15 \mathrm{~K}$

Imaginary Freq. $=-1180.4479 \mathrm{~cm}^{-1}$

Sp a B3LYP-D3 (BJ)/def2-TZVPP in toluene (SMD model)

SCF Done: E (RB3LYP) $=-2760.80864910$ a.u.

$\mathrm{Rh}, 0,1.9767497439,-0.3590457711,0.0960587049$

$0,0,-0.1975250222,0.4440544433,2.2910357635$

$0,0,0.8433732524,-1.3930901237,1.5811501461$

$C, 0,-0.0018974565,-0.8063440292,2.3127830472$

C, $0,-0.8569786224,-1.6494228407,3.2169497731$

$\mathrm{H}, 0,-1.7677765549,-1.9020890896,2.6635674326$

$\mathrm{H}, 0,-1.1420413439,-1.0906034124,4.1093075652$

$\mathrm{H}, \mathrm{O},-0.3464313749,-2.5748797276,3.4845979419$

$0,0,0.7606855694,-1.3997458697,-1.1807839236$

C, $0,-0.5297283977,-1.4000151881,-1.0352409006$

C, $0,-1.297136342,-0.2460344553,-0.8282384241$

C, $0,-1.2310201417,-2.6508239505,-1.1673883758$

C, $0,-2.716819072,-0.3364246182,-0.6412368938$

C, $0,-2.5837230325,-2.7496509296,-1.0625965244$

C, $0,-3.364264672,-1.5978910266,-0.7755910791$

$\mathrm{H}, \mathrm{O},-3.0732232054,-3.7124679839,-1.1731064094$

C, $0,-5.5203834967,-0.5993436509,-0.2808476247$

$\mathrm{C}, 0,-4.8831357879,0.6478309285,-0.1051889216$

$C, 0,-3.5266287219,0.7762169088,-0.2772931864$

C, $0,-4.7667594065,-1.6999814403,-0.6016141291$

$\mathrm{H}, \mathrm{O},-6.5939270846,-0.6869655715,-0.1466640423$

$\mathrm{H}, 0,-5.4703712583,1.5159481995,0.1794909009$

$\mathrm{H}, 0,-3.059173759,1.7401940379,-0.1124442467$

$\mathrm{H}, \mathrm{O},-5.2344653477,-2.6740766585,-0.7202739701$

C, $0,-0.6059673522,1.0604783833,-0.8436378572$

C, $0,-1.0998032679,2.0878768651,-1.6626441918$

C, $0,0.5338960911,1.3095203583,-0.0372045689$

C, $0,-0.5297320925,3.3529117765,-1.6522570965$

$\mathrm{H}, 0,-1.9440545657,1.8857746545,-2.3155349592$

C, $0,1.0386823006,2.6233057764,0.0027675692$

$\mathrm{H}, 0,0.3325898198,0.837711743,1.1901709441$

$\mathrm{C}, 0,0.5288681517,3.638936381,-0.789834116$

$\mathrm{H}, \mathrm{O},-0.9176783911,4.1250078365,-2.3100445201$

$\mathrm{H}, \mathrm{O}, 1.8423796246,2.8468102251,0.6980563311$

$\mathrm{H}, 0,0.9487441107,4.6391695792,-0.7476781523$

C, $0,3.7339236666,-1.4928541503,-0.4766880647$

C, $0,3.8100529649,-1.1866702628,0.9010822613$

$C, 0,3.8522179481,0.2403697756,1.0450222837$

C, $0,3.8531166522,0.8107196332,-0.2646767676$

C, $0,3.728582477,-0.2535655997,-1.2180444514$

C, 0, 4.101800998,2.2603172071,-0.5836361383

C, $0,3.7959937364,-0.1809100743,-2.7227022521$

$\mathrm{H}, 0,3.6331237098,-2.4793674079,-0.9083536465$ 
$\mathrm{H}, 0,3.7628914308,-1.9026733814,1.7106230532$

$\mathrm{H}, 0,3.9479341851,0.7912739337,1.9700815218$

$0,0,1.3353745374,-0.3739313233,-3.8719146848$

$0,0,6.6419828488,2.4076074683,0.4304415216$

$C, 0,3.1373097946,1.0255681057,-3.3553450531$

C, $0,1.8212413216,0.8814107445,-3.8995992642$

C, $0,1.1552114845,1.9616317703,-4.4220271912$

C, $0,1.7671374758,3.2397818667,-4.4422301768$

C, $0,1.0788742174,4.3777638319,-4.9377172763$

C, $0,1.6749903681,5.6134199456,-4.9456004168$

C, $0,2.9918778865,5.7691919021,-4.458347184$

C, $0,3.681896439,4.6856873519,-3.9741682218$

C, $0,3.0912261772,3.3955025306,-3.9527413541$

C, $0,3.7660396791,2.2492980017,-3.4196603291$

C, $0,-0.0009649596,-0.5925985023,-4.2689821253$

C, $0,5.303234241,2.3886493295,-1.4896620562$

C, $0,5.1430619718,2.3914257122,-2.855932775$

C, $0,6.2792952985,2.4880515037,-3.7230127144$

C, $0,6.1593489769,2.4898000492,-5.136942131$

C, $0,7.2705944312,2.5840015616,-5.9368908829$

C, $0,8.5566741573,2.6763632717,-5.3599563088$

C, $0,8.703524017,2.6694844662,-3.9958804071$

C, $0,7.5735377472,2.5742380957,-3.142693326$

C, $0,7.7185357591,2.5524338982,-1.7295049854$

C, $0,6.6123934542,2.4543063002,-0.9242536692$

$C, 0,7.9012044706,2.4719996886,1.0689841065$

$\mathrm{H}, 0,0.1513386353,1.8614273765,-4.8175716602$

$\mathrm{H}, 0,0.0655065903,4.2524779613,-5.3100774775$

$\mathrm{H}, \mathrm{O}, 1.1356170195,6.4756876236,-5.3258512989$

$\mathrm{H}, 0,3.4580590566,6.7494438286,-4.4663549983$

$\mathrm{H}, 0,4.6915656382,4.8108077376,-3.5969243044$

$\mathrm{H}, \mathrm{O},-0.6972198225,0.0044325023,-3.6704315627$

$\mathrm{H}, 0,-0.1978212931,-1.6503378663,-4.0968241752$

$\mathrm{H}, \mathrm{O},-0.1419794619,-0.3671358032,-5.3341746829$

$\mathrm{H}, 0,5.1728216731,2.4172809196,-5.582494938$

$\mathrm{H}, 0,7.1622721574,2.5866734578,-7.0168411224$

$\mathrm{H}, 0,9.4302290433,2.7497846548,-6.0004798505$

$\mathrm{H}, 0,9.6917457879,2.73568784,-3.5487257026$

$\mathrm{H}, 0,8.7180991681,2.6075465826,-1.3136961343$

$\mathrm{H}, 0,8.540362062,1.6281510221,0.7820153962$

$\mathrm{H}, 0,7.7016735058,2.4214563088,2.139886022$

$\mathrm{H}, 0,8.4178734132,3.412987736,0.8439174628$

$\mathrm{H}, 0,4.2656558186,2.8043017475,0.3479452749$

$\mathrm{H}, 0,3.2366910085,2.694848349,-1.0814520586$

$\mathrm{H}, 0,4.8582352181,-0.2011860345,-2.9940224535$

$\mathrm{H}, 0,3.3412453428,-1.0894686954,-3.1186943504$

$\mathrm{Cl}, 0,-0.2818905891,-4.0864298221,-1.4841074556$

\section{TS-3- $\left(S_{\mathrm{a}}, R_{\mathrm{Rh}}\right)$-Front}

Opt a wB97XD/SDD/6-31G $(\mathrm{d}, \mathrm{p})$ in toluene (SMD model)

SCF Done: E (RwB97XD) $=-2758.99484788$ a.u.

Zero-point correction $=0.710870$ Hartree/Particle

Sum of electronic and thermal Free Energies $=-2758.387050 \mathrm{a} . \mathrm{u}$. @ $358.15 \mathrm{~K}$

Imaginary Freq. $=-1177.2104 \mathrm{~cm}^{-1}$

Sp a B3LYP-D3 (BJ)/def2-TZVPP in toluene (SMD model)

SCF Done: E (RB3LYP) $=-2760.81204231 \mathrm{a} \cdot \mathrm{u}$. 
$\mathrm{Rh}, 0,0.9063823438,-1.8227164171,-0.0615989298$ $0,0,3.7852862638,-2.6635752407,-1.0409812455$ $0,0,1.9335429623,-1.7896080883,-1.9175040112$ C, $0,3.1355404487,-2.1693648092,-2.0076257667$ C, $0,3.8375262738,-1.9709121466,-3.3213987248$ $\mathrm{H}, \mathrm{O}, 4.3436640854,-1.0006385863,-3.2779454342$ $\mathrm{H}, 0,4.5919389033,-2.7434033492,-3.4766127178$ $\mathrm{H}, 0,3.1233315116,-1.9530342861,-4.1449041611$ $0,0,1.0695757224,0.2027370745,-0.2492561302$ C, $0,2.2151541703,0.7775258293,-0.4653640017$ C, $0,3.4062087867,0.4605873928,0.2064138982$ C, $0,2.2536316583,1.8557404123,-1.4211473947$ C, $0,4.634244239,1.1217294278,-0.1404600432$ C, $0,3.3903334454,2.5485579781,-1.6993972222$ C, $0,4.6176624018,2.1913981006,-1.0795330737$ $\mathrm{H}, 0,3.3727075193,3.3589141563,-2.4217180393$ C, $0,7.017877459,2.4841824338,-0.8667854334$ C, $0,7.0542335222,1.3889735637,0.0220280939$ C, $0,5.9023143826,0.7267850124,0.3711193986$ $\mathrm{C}, 0,5.8181818195,2.8663184439,-1.412010891$ $\mathrm{H}, 0,7.9335822069,3.0044766624,-1.1294310674$ $\mathrm{H}, 0,8.005621912,1.0552747455,0.4257600257$ $\mathrm{H}, 0,5.9641821681,-0.1278992196,1.0348252056$ $\mathrm{H}, 0,5.7715538819,3.6884039822,-2.1216960543$ C, $0,3.3555793654,-0.5219923972,1.3112241932$ C, $0,3.9485046478,-0.1997925385,2.5437136016$ C, $0,2.7309003837,-1.7848174268,1.1623350414$ C, $0,3.9641057729,-1.1032563007,3.5958558066$ $\mathrm{H}, \mathrm{O}, 4.3984527403,0.7795380273,2.6749313708$ C, $0,2.7954023877,-2.6942122794,2.2396145594$ $\mathrm{H}, 0,3.111129023,-2.3361026964,0.0137105014$ C, $0,3.3994881468,-2.3711815902,3.4431382272$ $\mathrm{H}, 0,4.4296304099,-0.8232267397,4.5365601039$ $\mathrm{H}, 0,2.3803023302,-3.6901912303,2.1085184628$ $\mathrm{H}, 0,3.4417311355,-3.0937629511,4.2525274481$ $\mathrm{Cl}, 0,0.771250612,2.2690899394,-2.2459726974$ $\mathrm{C}, 0,-0.2794486215,-2.8863508372,1.4136845759$ $C, 0,-0.0013844864,-3.7562755194,0.3078731439$ C, $0,-0.6157154186,-3.1642924419,-0.8378617356$ C, $0,-1.2382506379,-1.9374261369,-0.4679411953$ C, $0,-1.0418271345,-1.7743208486,0.9573939782$ C, $0,-1.9764081531,-0.9931342636,-1.3752025614$ $\mathrm{C}, 0,-1.6337909201,-0.7253287125,1.8617774322$ $\mathrm{H}, \mathrm{O}, 0.0415877219,-3.0282682684,2.4366198411$ $\mathrm{H}, 0,0.5353090729,-4.6935547983,0.3386761334$ $\mathrm{H}, \mathrm{O},-0.5554982493,-3.540302556,-1.8511246228$ $0,0,0.272374725,1.0604440969,2.6473637617$ $0,0,-3.9902325667,-2.6676993266,-2.1437270976$ C, $0,-1.6367083026,0.6925411659,1.3344727356$ C, $0,-0.6061274801,1.5851046119,1.7629430342$ $\mathrm{C}, 0,-0.5638474061,2.8778159548,1.30883253$ C, $0,-1.5377875324,3.3481628298,0.3923211793$ C, $0,-1.4905308002,4.6730569233,-0.1136226527$ C, $0,-2.422378206,5.1150875107,-1.0176144089$ C, $0,-3.4478024023,4.2500902782,-1.4595893134$ C, $0,-3.5201383587,2.9646880646,-0.9837918379$ C, $0,-2.5731433964,2.4809566016,-0.0436688035$ C, $0,-2.6063257786,1.1416098466,0.4666758702$ 
$C, 0,1.3409180052,1.8777978676,3.0745327441$

$\mathrm{C}, 0,-3.3884783663,-0.789713356,-0.8778468808$

$C, 0,-3.6871673221,0.212325355,0.0170347421$

C, $0,-5.0184135122,0.3605839949,0.5292010632$

$C, 0,-5.3627106701,1.3615659396,1.4746119479$

C, $0,-6.6491115275,1.4792238452,1.938873217$

$C, 0,-7.654032529,0.5984401556,1.4813915672$

C, $0,-7.3485369757,-0.3851184393,0.574979478$

C, $0,-6.0268572665,-0.5335183414,0.0801418094$

C, $0,-5.6983943388,-1.5632455474,-0.8414365267$

C, $0,-4.4105509147,-1.6981743055,-1.2934227834$

$C, 0,-4.946945519,-3.5906416497,-2.6212517739$

$\mathrm{H}, 0,0.2214243119,3.5577010889,1.6172278583$

$\mathrm{H}, 0,-0.6920116115,5.3299125795,0.2205442374$

$\mathrm{H}, \mathrm{O},-2.3701517652,6.1296626982,-1.4010549425$

$\mathrm{H}, \mathrm{O},-4.1780470179,4.6029995248,-2.1811320636$

$\mathrm{H}, 0,-4.3070496605,2.3038810321,-1.3317286915$

$\mathrm{H}, 0,1.973810169,2.1772565016,2.2310611622$

$\mathrm{H}, \mathrm{O}, 1.9255260423,1.273016333,3.7665897033$

$\mathrm{H}, 0,0.9758597909,2.7719844549,3.5962126422$

$\mathrm{H}, \mathrm{O},-4.5939738048,2.0413366056,1.8266384063$

$\mathrm{H}, \mathrm{0},-6.8964715644,2.2521311863,2.6597496817$

$\mathrm{H}, \mathrm{O},-8.6696359193,0.7005120678,1.8516166486$

$\mathrm{H}, \mathrm{O},-8.117192475,-1.0688354806,0.224670458$

$\mathrm{H}, \mathrm{O},-6.4843051962,-2.2380924773,-1.1609545649$

$\mathrm{H}, \mathrm{O},-5.3963667536,-4.1650024127,-1.8019044612$

$\mathrm{H}, \mathrm{O},-4.4092726063,-4.2717102595,-3.2818270599$

$\mathrm{H}, 0,-5.7395175802,-3.0895542728,-3.1901872029$

$\mathrm{H}, \mathrm{O},-1.9789837955,-1.4017077376,-2.3863887802$

$\mathrm{H}, 0,-1.4424961272,-0.03962774,-1.399168232$

$\mathrm{H}, \mathrm{O},-2.666991082,-1.0373661363,2.0564862635$

$\mathrm{H}, 0,-1.1011436734,-0.7594656103,2.8129738535$

\section{INT-4}

Opt a wB97XD/SDD/6-31G $(\mathrm{d}, \mathrm{p})$ in toluene (SMD model)

$\mathrm{SCF}$ Done: $\mathrm{E}(\mathrm{RwB} 97 \mathrm{XD})=-2759.01657323 \mathrm{a} \cdot \mathrm{u}$.

Zero-point correction $=0.716423$ Hartree/Particle

Sum of electronic and thermal Free Energies = -2758.402992 a.u. @ $358.15 \mathrm{~K}$

Sp a B3LYP-D3 (BJ)/def2-TZVPP in toluene (SMD model)

SCF Done: $\mathrm{E}(\mathrm{RB} 3 \mathrm{LYP})=-2760.82845188 \mathrm{a} \cdot \mathrm{u}$.

$\mathrm{Rh}, 0,0.8938481036,1.4867940809,-0.7275669746$

$0,0,1.9291023322,-1.8235979391,-0.3539953668$

$0,0,0.9139534883,-0.1525154563,0.7368321233$

C, $0,1.3519703237,-1.3041025559,0.7018175786$

C, $0,1.2995468822,-2.2005165316,1.8933044125$

$\mathrm{H}, \mathrm{O}, 2.2029911034,-2.0156172748,2.4848396822$

$\mathrm{H}, 0,1.2876178175,-3.2488435435,1.5959699792$

$\mathrm{H}, 0,0.427802434,-1.961471602,2.5018516976$

$0,0,2.4536500333,2.3242229512,0.3279069842$

$C, 0,3.4192162015,1.5675434151,0.759218356$

$\mathrm{C}, 0,4.0243889098,0.5650525429,-0.0092818323$

C, $0,3.9120896145,1.7810445243,2.0925154218$

C, $0,4.9431020166,-0.3522133975,0.6039858557$

C, $0,4.8668235139,0.9921747108,2.6567943815$

C $, 0,5.3827449506,-0.1188181347,1.9408797161$

$\mathrm{H}, 0,5.2099647892,1.1842979885,3.6687327368$ 
C, $0,6.7457335563,-2.1287267341,1.9091079244$ C, $0,6.2715643999,-2.4039930333,0.6080921535$ $\mathrm{C}, 0,5.4026844307,-1.5478066726,-0.0221375187$ $\mathrm{C}, 0,6.2960187511,-1.0090560775,2.5600269501$ $\mathrm{H}, 0,7.4414132204,-2.8071872878,2.3929018524$ $\mathrm{H}, 0,6.5899647074,-3.310263736,0.1008908633$ $\mathrm{H}, 0,5.0392801309,-1.8028648934,-1.0102188773$ $\mathrm{H}, 0,6.6232206297,-0.7904719976,3.5734538257$ $\mathrm{C}, 0,3.6625838856,0.4742478975,-1.4479511951$ C, $0,4.6813358953,0.2003641084,-2.378546841$ $\mathrm{C}, 0,2.3337550394,0.6385445198,-1.9208713625$ $\mathrm{C}, 0,4.4097152653,-0.0340388336,-3.7191728686$ $\mathrm{H}, 0,5.7100158873,0.1682296548,-2.0328010377$ C, $0,2.0810443823,0.3417483283,-3.2713059326$ $\mathrm{H}, 0,1.9898026533,-1.1297165814,-1.0511346695$ C, $0,3.0918340943,-0.0056636458,-4.1628470614$ $\mathrm{H}, 0,5.2221194403,-0.2508590636,-4.406359255$ $\mathrm{H}, 0,1.0598143872,0.3606115084,-3.6354301826$ $\mathrm{H}, 0,2.850236115,-0.229616988,-5.1983292763$ $\mathrm{C}, 0,-0.3665997127,2.2627397948,-2.3211846305$ C, $0,0.145448789,3.3161002508,-1.5050548012$ $\mathrm{C}, 0,-0.4702716445,3.1916208891,-0.2013586519$ C, $0,-1.2370056437,2.0239245518,-0.1862420423$ C, $0,-1.1658712249,1.4093707836,-1.5164387405$ C, $0,-1.9335330669,1.4431298012,1.0135510044$ $\mathrm{C}, 0,-1.9874195662,0.2563754852,-2.0361426135$ $\mathrm{H}, \mathrm{O},-0.1435427817,2.1147632097,-3.3683768111$ $\mathrm{H}, 0,0.7985717945,4.1148124176,-1.8278635076$ $\mathrm{H}, \mathrm{O},-0.2710448166,3.8221268283,0.6543630231$ $0,0,-0.2945505217,-1.8202897717,-2.5582357873$ $0,0,-3.8080420467,3.3665359708,1.4679252217$ C, $0,-1.9783515599,-0.9736176814,-1.1587120357$ $\mathrm{C}, 0,-1.0658472045,-2.0319710397,-1.4644362083$ $\mathrm{C}, 0,-1.011146127,-3.1622262337,-0.6892301067$ $\mathrm{C}, 0,-1.8369366908,-3.2825470247,0.4585141326$ $\mathrm{C}, 0,-1.7539142775,-4.4173019776,1.3071736538$ $C, 0,-2.5388425671,-4.5151059411,2.4280356906$ $\mathrm{C}, 0,-3.446924845,-3.4822158168,2.7506610915$ C, $0,-3.5518823409,-2.3762669187,1.9446827143$ C, $0,-2.7533024618,-2.2463033779,0.7782360405$ $\mathrm{C}, 0,-2.8221187533,-1.0975272976,-0.076876679$ $\mathrm{C}, 0,0.5151867468,-2.8807313449,-3.0302367241$ $\mathrm{C}, 0,-3.3947665623,1.1806372647,0.7357783695$ $\mathrm{C}, 0,-3.818059131,-0.0197547728,0.212823608$ $C, 0,-5.2042179122,-0.2391898632,-0.0857081575$ $\mathrm{C}, 0,-5.6799176373,-1.4471584824,-0.6594688276$ $\mathrm{C}, 0,-7.0145223828,-1.6228908492,-0.9275195372$ $C, 0,-7.9400818142,-0.5964305736,-0.6373696354$ $\mathrm{C}, 0,-7.5084042656,0.5857953337,-0.0914970223$ $\mathrm{C}, 0,-6.134416533,0.7977282111,0.1932402097$ $\mathrm{C}, 0,-5.6792475691,2.0282767608,0.7365601299$ $\mathrm{C}, 0,-4.343931343,2.2209921822,0.9832063102$ $\mathrm{C}, 0,-4.6827298805,4.431426555,1.7795822818$ $\mathrm{H}, 0,-0.3235366719,-3.9678745229,-0.9191478099$ $\mathrm{H}, \mathrm{0},-1.051765646,-5.2075254376,1.0547461689$ $\mathrm{H}, \mathrm{O},-2.4627292318,-5.3861236112,3.0716922869$ $\mathrm{H}, \mathrm{O},-4.0614162295,-3.5636585063,3.6415552549$ $\mathrm{H}, \mathrm{O},-4.2473332372,-1.5839754888,2.1999222941$ 
$\mathrm{H}, \mathrm{0}, 1.2669307417,-3.1771461364,-2.2919929192$

$\mathrm{H}, 0,1.0214009742,-2.4991633092,-3.9171923754$

$\mathrm{H}, \mathrm{O},-0.0949492682,-3.7508053968,-3.3015286935$

$\mathrm{H}, \mathrm{O},-4.9746376618,-2.239307319,-0.8859492345$

$\mathrm{H}, \mathrm{0},-7.3609288439,-2.5537528094,-1.3652571869$

$\mathrm{H}, \mathrm{O},-8.9941813375,-0.7442899023,-0.8513289647$

$\mathrm{H}, 0,-8.2152457776,1.381633302,0.127231614$

$\mathrm{H}, \mathrm{O},-6.4081589969,2.8062637002,0.9331466438$

$\mathrm{H}, \mathrm{O},-5.2245103257,4.7812662176,0.8923223613$

$\mathrm{H}, \mathrm{O},-4.0539855207,5.2399535091,2.1540523783$

$\mathrm{H}, 0,-5.4015813643,4.1451035572,2.5570792263$

$\mathrm{H}, 0,-1.8218098772,2.1344272745,1.8495255185$

$\mathrm{H}, 0,-1.435866608,0.5095620131,1.2897635261$

$\mathrm{H}, 0,-3.0186242126,0.6071188146,-2.1547657693$

$\mathrm{H}, 0,-1.6172972106,-0.0029561967,-3.0290716726$

$\mathrm{Cl}, 0,3.2245981639,3.0996416344,3.0158534383$

\section{INT-5- $\left(R_{\mathrm{a}}, R \boldsymbol{e}_{\mathrm{Rh}}\right)$}

Opt \& wB97XD/SDD/6-31G(d,p) in toluene (SMD model)

$\mathrm{SCF}$ Done: $\mathrm{E}(\mathrm{RwB} 97 \mathrm{XD})=-2529.97122052 \mathrm{a} \cdot \mathrm{u}$.

Zero-point correction $=0.651980$ Hartree/Particle

Sum of electronic and thermal Free Energies = -2529.413062 a.u. @ $358.15 \mathrm{~K}$

$\mathrm{Sp}$ a B3LYP-D3 (BJ)/def2-TZVPP in toluene (SMD model)

$\mathrm{SCF}$ Done: $\mathrm{E}(\mathrm{RB} 3 \mathrm{LYP})=-2531.61134824 \mathrm{a} \cdot \mathrm{u}$.

$\mathrm{Rh}, 0,0.8415346783,-2.3895573864,-0.3424782474$

$0,0,1.3262067213,-0.9985688421,-1.6619136032$

C, $0,2.1020642636,0.0384399097,-1.4890882264$

$\mathrm{C}, 0,3.1856569601,0.077319006,-0.6041064121$

C, $0,1.8137243942,1.1963355304,-2.2898687201$

C, $0,3.8469168788,1.3412398835,-0.3665658396$

$\mathrm{C}, 0,2.4947938618,2.3606640816,-2.151903194$

C, $0,3.5007746172,2.4753758885,-1.1566861034$

$\mathrm{H}, \mathrm{O}, 2.2395984573,3.2233092657,-2.7580625432$

C, $0,5.0112753211,3.9049058888,0.0987826872$

C, $0,5.3286524007,2.8058434803,0.9211444447$

$\mathrm{C}, 0,4.7706694321,1.5700946329,0.6937953385$

C, $0,4.1076011806,3.7329451036,-0.9189809184$

$\mathrm{H}, 0,5.4641268172,4.8745534211,0.2794966828$

$\mathrm{H}, 0,6.0165933052,2.9339863183,1.7515957952$

$\mathrm{H}, 0,5.0288168613,0.7609993788,1.3647198426$

$\mathrm{H}, 0,3.821895585,4.5693222428,-1.5515980336$

$\mathrm{C}, 0,3.5817231525,-1.1692989422,0.0850879985$

C, $0,4.951785893,-1.3907838175,0.3478510891$

$C, 0,2.6555882647,-2.1747068429,0.4772583151$

$\mathrm{C}, 0,5.3952846326,-2.4737288017,1.0865056215$

$\mathrm{H}, 0,5.684049951,-0.7030495649,-0.0610512204$

$\mathrm{C}, 0,3.1331708363,-3.2419495929,1.2680911518$

$\mathrm{C}, 0,4.4734482067,-3.3922820502,1.5886780974$

$\mathrm{H}, 0,6.4585817283,-2.606905426,1.2628107478$

$\mathrm{H}, 0,2.4337151607,-3.9985236771,1.6112461501$

$\mathrm{H}, 0,4.8033996465,-4.2366144162,2.1872331539$

C, $0,-0.2896388321,-3.5142075276,1.1274354776$

$C, 0,-0.2360046558,-4.2551821269,-0.0723555952$

C, $0,-0.9586271564,-3.517257429,-1.0947634416$

C, $0,-1.3849815049,-2.3025086669,-0.5559842259$

$\mathrm{C}, 0,-0.9266796559,-2.2589124466,0.835907941$ 
$C, 0,-2.0114580892,-1.1658282783,-1.3202988014$

$\mathrm{C}, 0,-1.264890698,-1.2068758681,1.8577581431$

$\mathrm{H}, 0,0.114451041,-3.7970422854,2.0895527195$

$\mathrm{H}, \mathrm{O}, 0.2215381536,-5.2266520131,-0.2050468215$

$\mathrm{H}, \mathrm{0},-1.0735947425,-3.8225615706,-2.1264232266$

$0,0,1.0917905923,-0.1712490372,2.6768845481$

$0,0,-4.5375018555,-2.0835635956,-1.7642312542$

$\mathrm{C}, 0,-0.8586390834,0.1838811917,1.4319978662$

$\mathrm{C}, 0,0.4218103316,0.6637670416,1.8481084638$

$\mathrm{C}, 0,0.8785515846,1.8902584805,1.4379421549$

$\mathrm{C}, 0,0.0830643688,2.695501256,0.5826043639$

C, $0,0.5468904039,3.9574785947,0.1282936475$

C, $0,-0.2168540689,4.7251900543,-0.7128107203$

C, $0,-1.4841088276,4.2674749953,-1.1388078367$

C, $0,-1.9636369482,3.0575813989,-0.7045559459$

C, $0,-1.1985361484,2.2427233582,0.1701290699$

$\mathrm{C}, 0,-1.662159989,0.9692537881,0.6356182124$

C, $0,2.3693466469,0.222610762,3.1305227829$

$\mathrm{C}, 0,-3.1945156181,-0.5413423596,-0.6199344751$

$C, 0,-3.033559331,0.4871724921,0.2809712455$

$\mathrm{C}, 0,-4.1713329655,1.0685607874,0.9351287883$

$\mathrm{C}, 0,-4.0507951474,2.1103525039,1.8921021396$

$\mathrm{C}, 0,-5.1618301019,2.6521517352,2.4887012711$

$\mathrm{C}, 0,-6.4500265998,2.1750064268,2.1610387032$

C, $0,-6.5971515147,1.1592463189,1.2509795857$

$\mathrm{C}, 0,-5.4664403245,0.5766123117,0.6213376254$

$\mathrm{C}, 0,-5.6108284596,-0.4922765559,-0.3019359949$

C, $0,-4.5040371203,-1.0451576529,-0.8935038729$

$C, 0,-5.7953467543,-2.6406154788,-2.0838929506$

$\mathrm{H}, 0,1.8525192445,2.2606056779,1.737115471$

$\mathrm{H}, 0,1.5294514166,4.2942253043,0.4471364889$

$\mathrm{H}, 0,0.1528941861,5.68590744,-1.0587600954$

$\mathrm{H}, \mathrm{O},-2.0788888362,4.8756677158,-1.8130631095$

$\mathrm{H}, \mathrm{O},-2.936165875,2.7084761142,-1.0354607762$

$\mathrm{H}, 0,3.0425856683,0.4267802285,2.2951067551$

$\mathrm{H}, 0,2.760427664,-0.6166012146,3.7063725743$

$\mathrm{H}, 0,2.306582584,1.1099395707,3.7735085213$

$\mathrm{H}, \mathrm{O},-3.064278732,2.4805393483,2.149039263$

$\mathrm{H}, 0,-5.0510395659,3.4498081086,3.2164222827$

$\mathrm{H}, 0,-7.3237602494,2.6120201427,2.6348651318$

$\mathrm{H}, 0,-7.5855064239,0.783295951,1.0003557117$

$\mathrm{H}, 0,-6.6083266058,-0.8591457177,-0.5157802896$

$\mathrm{H}, 0,-6.2957308569,-3.0407143697,-1.1935786369$

$\mathrm{H}, \mathrm{O},-5.598086115,-3.4568446748,-2.7797244389$

$\mathrm{H}, \mathrm{O},-6.4499686377,-1.9055360991,-2.5678309677$

$\mathrm{H}, \mathrm{O},-2.3111460153,-1.5375406783,-2.3010709061$

$\mathrm{H}, 0,-1.243811477,-0.4050818608,-1.4890681641$

$\mathrm{H}, 0,-2.3457910062,-1.2505265039,2.0278000457$

$\mathrm{H}, \mathrm{0},-0.7707492466,-1.4655431686,2.7940815883$

$\mathrm{Cl}, 0,0.525194618,1.0874203772,-3.4654535174$

\section{INT-5- $\left(S_{\mathrm{a}}, R e_{\mathrm{Rh}}\right)$}

Opt a wB97XD/SDD/6-31G(d,p) in toluene (SMD model)

$\mathrm{SCF}$ Done: $\mathrm{E}(\mathrm{RwB} 97 \mathrm{XD})=-2529.96387533 \mathrm{a} \cdot \mathrm{u}$.

Zero-point correction $=0.651487$ Hartree/Particle

Sum of electronic and thermal Free Energies = -2529.408050 a.u. @ $358.15 \mathrm{~K}$

$\mathrm{Sp}$ a B3LYP-D3 (BJ)/def2-TZVPP in toluene (SMD model) 
SCF Done: E (RB3LYP) $=-2531.60503637$ a.u.

$\mathrm{Rh}, 0,0.8208538504,-1.3602763337,0.8029926129$

$0,0,1.1638166162,0.3903667521,-0.0541649679$

C, $0,2.305587825,0.8805529811,-0.4629540785$

$\mathrm{C}, 0,3.5542413904,0.5314097315,0.064124572$

C, $0,2.2504545296,1.8686388666,-1.5070396305$

$\mathrm{C}, 0,4.7498652821,0.9868575078,-0.604682912$

$\mathrm{C}, 0,3.3694420392,2.414005696,-2.0514023814$

C, $0,4.6511834382,1.9584808673,-1.6404126508$

$\mathrm{H}, 0,3.2921809761,3.1642404517,-2.8318659072$

$\mathrm{C}, 0,7.0549379043,1.9276953454,-1.9780771598$

$\mathrm{C}, 0,7.1581090736,0.9104981193,-1.0072544244$

C, $0,6.0440684616,0.4552191134,-0.3440008162$

$\mathrm{C}, 0,5.8173882299,2.4284238457,-2.29342869$

$\mathrm{H}, 0,7.9422990181,2.2881065329,-2.4888021595$

$\mathrm{H}, 0,8.1263306071,0.4694905309,-0.789465711$

$\mathrm{H}, 0,6.1531394848,-0.3522814174,0.3697831282$

$\mathrm{H}, 0,5.7080955803,3.183986764,-3.0669913254$

$\mathrm{C}, 0,3.6084388637,-0.2828759116,1.2959872889$

C, $0,4.6197905488,-0.0033779864,2.2423583193$

$C, 0,2.6561285633,-1.286354841,1.6097380385$

$\mathrm{C}, 0,4.7665809556,-0.7446890969,3.401217122$

$\mathrm{H}, 0,5.2874883507,0.8340067723,2.0687685388$

$\mathrm{C}, 0,2.8711078585,-2.0670800265,2.7670800439$

C, $0,3.9065522361,-1.8148617059,3.65321545$

$\mathrm{H}, 0,5.5497744052,-0.4888323912,4.1088627361$

$\mathrm{H}, 0,2.1777057594,-2.8700456261,2.9999787156$

$\mathrm{H}, 0,4.0265625819,-2.422141003,4.545853346$

$\mathrm{Cl}, 0,0.6777066013,2.3719578809,-2.0784266828$

$\mathrm{C}, 0,-0.3923267391,-2.8442899113,1.805881761$

$\mathrm{C}, 0,0.3047177636,-3.4437904343,0.7377056166$

$\mathrm{C}, 0,-0.1244529233,-2.7745665926,-0.4682129842$

C, $0,-1.1723843711,-1.8440511614,-0.1497621639$

C, $0,-1.2991966116,-1.8446769182,1.2581017574$

C, $0,-1.8501161008,-0.9171466147,-1.1284793419$

$\mathrm{C}, 0,-2.1654723227,-0.9409795327,2.0914444947$

$\mathrm{H}, \mathrm{O},-0.3000280087,-3.0795756841,2.8576324501$

$\mathrm{H}, 0,1.0490379875,-4.2249926824,0.7994354764$

$\mathrm{H}, 0,0.242969737,-2.9789902143,-1.4658091885$

$0,0,-0.2004467108,0.6348783902,3.1180329373$

$0,0,-3.6382953446,-2.5961084842,-2.3244352492$

$\mathrm{C}, 0,-2.0508984878,0.5110004464,1.6808860927$

$\mathrm{C}, 0,-1.0102275392,1.3022650419,2.2565521175$

$\mathrm{C}, 0,-0.8621645517,2.6207391652,1.9171288902$

C, $0,-1.7186723223,3.2092251102,0.950801116$

$\mathrm{C}, 0,-1.5690906186,4.5678619403,0.5701644845$

$\mathrm{C}, 0,-2.3671615944,5.1187543109,-0.3996598612$

$\mathrm{C}, 0,-3.3512054834,4.3333784894,-1.0399142993$

$\mathrm{C}, 0,-3.521310804,3.0173508447,-0.6895542018$

C, $0,-2.7207420113,2.4237876938,0.3209093863$

$\mathrm{C}, 0,-2.8650084485,1.0532348435,0.7126695336$

$\mathrm{C}, 0,1.0054096924,1.2651203475,3.5154336662$

$\mathrm{C}, 0,-3.3350245596,-0.7837803427,-0.8684151647$

$\mathrm{C}, 0,-3.8316041805,0.161169667,0.0039211709$

$\mathrm{C}, 0,-5.2382038975,0.2565019649,0.2595166758$

$\mathrm{C}, 0,-5.7828809522,1.1954958198,1.1738082193$

C, $0,-7.1361390787,1.2604918996,1.3933204467$ 
$C, 0,-8.0102834746,0.3856533627,0.7104820603$

$\mathrm{C}, 0,-7.5111871118,-0.5393182387,-0.1711768185$

C, $0,-6.1162816267,-0.6320710923,-0.4162416106$

$\mathrm{C}, 0,-5.5886270648,-1.6021263944,-1.3086109451$

C, $0,-4.2351131469,-1.6855012893,-1.5168303181$

$C, 0,-4.4633683552,-3.5038244446,-3.0246949022$

$\mathrm{H}, \mathrm{O},-0.0752342136,3.2280863129,2.3487642232$

$\mathrm{H}, 0,-0.7996172119,5.1628197979,1.0543991803$

$\mathrm{H}, \mathrm{O},-2.2379097132,6.158453632,-0.6844737863$

$\mathrm{H}, \mathrm{O},-3.9724935541,4.772837229,-1.8139824808$

$\mathrm{H}, 0,-4.2760476571,2.4178649376,-1.1874767769$

$\mathrm{H}, 0,1.5835718241,1.590203967,2.644109924$

$\mathrm{H}, 0,1.5740452945,0.5139761302,4.0644180392$

$\mathrm{H}, 0,0.8071742158,2.1214736567,4.1714526848$

$\mathrm{H}, 0,-5.1137173349,1.868858259,1.6991677489$

$\mathrm{H}, \mathrm{O},-7.537687988,1.9856859629,2.0940332076$

$\mathrm{H}, 0,-9.0796835884,0.4452973392,0.8887300389$

$\mathrm{H}, \mathrm{O},-8.1796314347,-1.2180972293,-0.6940586143$

$\mathrm{H}, \mathrm{O},-6.2787254815,-2.2745230661,-1.8053881764$

$\mathrm{H}, \mathrm{O},-5.0329909773,-4.1420188479,-2.3380110559$

$\mathrm{H}, \mathrm{O},-3.7937809332,-4.1277477824,-3.6177393956$

$\mathrm{H}, \mathrm{O},-5.1562748882,-2.9820962381,-3.6959464955$

$\mathrm{H}, \mathrm{O},-1.6852830864,-1.2993888614,-2.1368466762$

$\mathrm{H}, \mathrm{O},-1.3704275947,0.0651397123,-1.0727390129$

$\mathrm{H}, \mathrm{O},-3.2035602346,-1.2747319226,1.9977896849$

$\mathrm{H}, \mathrm{O},-1.8777443818,-1.0528656735,3.1381208497$

\section{INT-5- $\left(R_{\mathrm{a}}, S i_{\mathrm{Rh}}\right)$}

Opt \& $\mathrm{wB} 97 \mathrm{XD} / \mathrm{SDD} / 6-31 \mathrm{G}(\mathrm{d}, \mathrm{p})$ in toluene (SMD model)

$\mathrm{SCF}$ Done: $\mathrm{E}(\mathrm{RwB} 97 \mathrm{XD})=-2529.96248193 \mathrm{a} \cdot \mathrm{u}$.

Zero-point correction $=0.651651$ Hartree/Particle

Sum of electronic and thermal Free Energies = -2529.406306 a.u. @ $358.15 \mathrm{~K}$

$\mathrm{Sp}$ a B3LYP-D3 (BJ)/def2-TZVPP in toluene (SMD model)

$\mathrm{SCF}$ Done: E (RB3LYP) $=-2531.60340258 \mathrm{a} \cdot \mathrm{u}$.

$\mathrm{Rh}, 0,1.5354656952,0.1832975497,-0.9455111833$

$0,0,0.0916442276,-0.9283923757,-1.7550278964$

C, $0,-1.1021741737,-1.1326782244,-1.2614043687$

C, $0,-1.804185414,-0.2112493933,-0.4758777942$

$C, 0,-1.7373441631,-2.3830857989,-1.5778311272$

$\mathrm{C}, 0,-3.0278780369,-0.6208797288,0.1684331557$

C, $0,-2.9578674164,-2.7285017376,-1.0898911206$

C, $0,-3.6211225901,-1.869405868,-0.1742429982$

$\mathrm{H}, \mathrm{O},-3.4098524539,-3.6777080493,-1.3592454884$

C, $0,-5.4411966107,-1.5005003024,1.3886398893$

$\mathrm{C}, 0,-4.8213377502,-0.3008420833,1.7958991543$

C, $0,-3.655094895,0.1241009247,1.2071207528$

$\mathrm{C}, 0,-4.8382629602,-2.2729207001,0.4291808209$

$\mathrm{H}, 0,-6.3696623321,-1.8204604985,1.8509674176$

$\mathrm{H}, 0,-5.2621969786,0.2889559787,2.5941067434$

$\mathrm{H}, \mathrm{O},-3.1868463131,1.0324097416,1.5664183493$

$\mathrm{H}, \mathrm{O},-5.2754129136,-3.2214833847,0.1282215107$

C, $0,-1.2669220591,1.1592713659,-0.3458856541$

C, $0,-2.1724734334,2.2408516133,-0.3031210202$

$\mathrm{C}, 0,0.1208892685,1.4471814257,-0.3133347348$

C, $0,-1.7495555409,3.5474159028,-0.1269943235$

$\mathrm{H}, \mathrm{0},-3.231600121,2.0462279434,-0.4351923006$ 
$C, 0,0.5199376837,2.7784927437,-0.0752085361$ $\mathrm{C}, 0,-0.3907186476,3.8200995869,0.0225598229$ $\mathrm{H}, 0,-2.4787060316,4.3519063768,-0.1083867904$ $\mathrm{H}, \mathrm{O}, 1.5774329341,3.0114616373,-0.0000516083$ $\mathrm{H}, \mathrm{O},-0.0443435274,4.8367789382,0.1841638514$ $\mathrm{C}, 0,3.2669593648,-1.2743812417,-0.9543170012$ $\mathrm{C}, 0,2.8198952314,-0.8496440962,0.3515021368$ $\mathrm{C}, 0,3.1045295332,0.5562682246,0.4808176071$ C, $0,3.5646876433,1.0217106166,-0.7691535276$ C, $0,3.6772853867,-0.1431448001,-1.6616652$ C, $0,3.9809311454,2.4379928479,-1.0839886966$ C, $0,4.1501886493,-0.1235792855,-3.0898063293$ $\mathrm{H}, 0,3.1712569876,-2.2722726574,-1.3602456729$ $\mathrm{H}, 0,2.4401032633,-1.4946448615,1.1324613752$ $\mathrm{H}, 0,2.9378695415,1.1615372837,1.3614330526$ $0,0,1.7926157642,-0.3353771547,-4.4271268268$ $0,0,6.251059931,2.6247432818,0.4281816711$ $\mathrm{C}, 0,3.603841643,1.042610741,-3.883170462$ C, $0,2.3515070611,0.8873351979,-4.5545936684$ $C, 0,1.7969656996,1.9311668169,-5.2528203083$ C, $0,2.4649287545,3.1815303526,-5.3219587323$ C, $0,1.9093616087,4.2732720548,-6.0393290556$ C, $0,2.5511302556,5.4846352254,-6.0844345747$ C, $0,3.7753970315,5.6666269849,-5.4032838107$ C, $0,4.3354016842,4.6300585048,-4.6995650582$ $\mathrm{C}, 0,3.7021542173,3.3607716878,-4.6465539876$ C, $0,4.2545263037,2.2550877847,-3.9228118273$ $C, 0,0.4578990931,-0.509429039,-4.866149921$ C, $0,5.3473933678,2.5036871767,-1.7295426921$ C, $0,5.4879818627,2.4315675993,-3.0980000746$ C, $0,6.7840344448,2.4712785938,-3.7043857345$ C, $0,6.9702470652,2.3788227643,-5.108481464$ C, $0,8.2282841034,2.423365377,-5.6549027329$ C, $0,9.3615210793,2.5564736065,-4.821689044$ C, $0,9.2125397095,2.6373925399,-3.4603698242$ C, $0,7.924503575,2.593442255,-2.8657490613$ C, $0,7.7618248672,2.656964016,-1.4564269356$ C, $0,6.5076715547,2.6000716283,-0.9023985735$ C, $0,7.3480698479,2.7198733799,1.3139466757$ $\mathrm{H}, 0,0.8452059467,1.8232627021,-5.7600408148$ $\mathrm{H}, 0,0.961993759,4.1325359848,-6.5523673939$ $\mathrm{H}, 0,2.1150337982,6.3108797808,-6.6376369675$ $\mathrm{H}, 0,4.2731136526,6.6307536575,-5.4363848954$ $\mathrm{H}, 0,5.2744695667,4.7756588098,-4.1758148685$ $\mathrm{H}, 0,-0.2115483463,0.2094268401,-4.381768032$ $\mathrm{H}, 0,0.1708941034,-1.5155354789,-4.5657716883$ $\mathrm{H}, 0,0.3837703778,-0.4128674256,-5.9566860427$ $\mathrm{H}, 0,6.101490911,2.2749151563,-5.7502041224$ $\mathrm{H}, 0,8.3551510121,2.3544435387,-6.7306430058$ $\mathrm{H}, 0,10.3528522024,2.5913814716,-5.2630001617$ $\mathrm{H}, \mathrm{O}, 10.0823610239,2.733309323,-2.8161089224$ $\mathrm{H}, 0,8.6486349179,2.7379029545,-0.8382153501$ $\mathrm{H}, 0,8.0265987246,1.8647930865,1.207288106$ $\mathrm{H}, 0,6.9262469971,2.7172882617,2.3196353114$ $\mathrm{H}, 0,7.9077442899,3.6506762003,1.1615534422$ $\mathrm{H}, 0,3.9835148356,3.0095805067,-0.1542202565$ $\mathrm{H}, 0,3.2469130534,2.8981707909,-1.7495892444$ $\mathrm{H}, 0,5.2438769098,-0.0806437329,-3.0841601385$ 
$\mathrm{H}, 0,3.8602654731,-1.0635735934,-3.5613500886$

$\mathrm{Cl}, 0,-0.8878526671,-3.4946339941,-2.630843831$

\section{INT-5- $\left(S_{\mathrm{a}}, S i_{\mathrm{Rh}}\right)$}

Opt \& wB97XD/SDD/6-31G $(\mathrm{d}, \mathrm{p})$ in toluene (SMD model)

$\mathrm{SCF}$ Done: $\mathrm{E}(\mathrm{RwB} 97 \mathrm{XD})=-2529.96018951 \mathrm{a} \cdot \mathrm{u}$.

Zero-point correction $=0.651975$ Hartree/Particle

Sum of electronic and thermal Free Energies $=-2529.401721 \mathrm{a} . \mathrm{u}$. d $358.15 \mathrm{~K}$ Sp a B3LYP-D3 (BJ)/def2-TZVPP in toluene (SMD model)

SCF Done: E (RB3LYP) $=-2531.59974133$ a.u.

$\mathrm{Rh}, 0,1.1146783066,-1.4440609809,-0.7814703647$

$0,0,3.0561570844,-1.9206740836,-0.9226914009$

C, $0,4.0280379403,-1.2293408711,-0.3841110772$

C, $0,4.0625093066,0.1682399205,-0.3370851633$

C, $0,5.1365619702,-1.9594439875,0.1650435419$

C, $0,5.1088827504,0.8320622509,0.3951838034$

$C, 0,6.1854154907,-1.343967782,0.7734536071$

C, $0,6.1862813307,0.0672620187,0.9289778086$

$\mathrm{H}, 0,7.008780491,-1.9269163815,1.173767419$

C, 0,7.215001528,2.0581497685,1.8638486298

C, $0,6.1245369517,2.8186936743,1.3926765328$

C, $0,5.1079389948,2.2270007412,0.6829776626$

C, $0,7.2328845602,0.7053563022,1.6402774613$

$\mathrm{H}, 0,8.0187596136,2.5371894491,2.4138507206$

$\mathrm{H}, 0,6.0819252383,3.8839788855,1.5998530239$

$\mathrm{H}, 0,4.2752276832,2.8367298455,0.3553688009$

$\mathrm{H}, 0,8.0477648688,0.0940750019,2.0191970994$

C, $0,3.0127369241,0.9012963357,-1.0737492801$

C, $0,3.3630083849,2.0645595179,-1.7852598632$

C, $0,1.6691157603,0.4490384442,-1.1367327132$

C, 0,2.4208797795,2.815219841,-2.4739139593

$\mathrm{H}, 0,4.4044593408,2.3672866348,-1.8194385676$

C, $0,0.7286918877,1.2533020561,-1.8049652545$

C, $0,1.0853965684,2.4219662505,-2.4665096012$

$\mathrm{H}, \mathrm{0}, 2.7320166012,3.7005524487,-3.0205820389$

$\mathrm{H}, 0,-0.3138232617,0.9561650432,-1.8170690854$

$\mathrm{H}, 0,0.3290113276,3.009384961,-2.9784648129$

$\mathrm{Cl}, 0,5.1104790818,-3.7051620534,0.046680561$

$C, 0,0.380907924,-2.3443784495,0.9684084159$

C, $0,0.3947753997,-3.4221055446,0.0172446114$

$C, 0,-0.4418351572,-3.0378005133,-1.0323319172$

$C, 0,-1.0764428904,-1.7662716149,-0.7166309011$

$C, 0,-0.6105601994,-1.367797182,0.5626810782$

C, $0,-2.1985471841,-1.1441878958,-1.5061051191$

$C, 0,-1.1334819602,-0.2962252627,1.48179184$

$\mathrm{H}, 0,0.9399461129,-2.3066565243,1.8948068436$

$\mathrm{H}, 0,1.0501187894,-4.2810019228,0.0418785346$

$\mathrm{H}, 0,-0.586669609,-3.5733237577,-1.9622371054$

$0,0,0.496356969,1.878937068,1.5609186336$

$0,0,-3.9356345065,-3.243833268,-1.4558299766$

C, $0,-1.5518336394,1.0018692368,0.8334409377$

$C, 0,-0.6554728402,2.1135516265,0.8944702618$

C, $0,-0.9770869891,3.307952505,0.3022606334$

C, $0,-2.1988721265,3.4518670528,-0.4019944443$

C, $0,-2.5261732479,4.6635576036,-1.0656658237$

C, $0,-3.7035097541,4.7903382751,-1.757735238$ 
$C, 0,-4.6098231761,3.7082958934,-1.822258146$ $C, 0,-4.3191008939,2.5245288354,-1.191612507$ C, $0,-3.1089927643,2.362965407,-0.4672964732$ C, $0,-2.7624293015,1.1369358742,0.1887984488$ C, 0,1.5223726901,2.8478409727,1.4753415564 C, $0,-3.4492494105,-1.0958213897,-0.6582750413$ $C, 0,-3.7133669742,-0.0177041548,0.1553955349$ $C, 0,-4.8731255131,-0.008717582,1.0001358784$ C, $0,-5.1790919792,1.0758604004,1.8630340047$ C, $0,-6.2977021812,1.050050333,2.6580824639$ C, $0,-7.1648019789,-0.0643876849,2.6308968135$ C, $0,-6.8905232621,-1.1310729787,1.8128936536$ C, $0,-5.7420421245,-1.132472848,0.979261284$ C, $0,-5.4427913119,-2.2415434175,0.1437086163$ C, $0,-4.3217465327,-2.2274883153,-0.6455588$ C, $0,-4.748243544,-4.3991213196,-1.5043040558$ $\mathrm{H}, 0,-0.2946605156,4.1491756873,0.3307109923$ $\mathrm{H}, \mathrm{O},-1.8233884501,5.4909077103,-1.0169529856$ $\mathrm{H}, \mathrm{O},-3.9403374999,5.7220261732,-2.2624168029$ $\mathrm{H}, \mathrm{O},-5.5384654362,3.8147300781,-2.3740134359$ $\mathrm{H}, \mathrm{O},-5.0159329471,1.6949055901,-1.2475921699$ $\mathrm{H}, 0,1.7679387943,3.0666095132,0.4299620664$ $\mathrm{H}, 0,2.39029769,2.4128861449,1.9709880378$ $\mathrm{H}, \mathrm{O}, 1.2404424862,3.7744137758,1.9914096322$ $\mathrm{H}, \mathrm{O},-4.5157758738,1.9336220381,1.8904568453$ $\mathrm{H}, \mathrm{O},-6.5175621669,1.8888371333,3.310882255$ $\mathrm{H}, \mathrm{O},-8.0475905193,-0.0765039333,3.2627825105$ $\mathrm{H}, 0,-7.5515951731,-1.9933289457,1.7918791153$ $\mathrm{H}, 0,-6.1152969146,-3.0915641411,0.1549546526$ $\mathrm{H}, \mathrm{O},-4.822077898,-4.8805560098,-0.5215935753$ $\mathrm{H}, \mathrm{O},-4.2631329517,-5.08263543,-2.2019764314$ $\mathrm{H}, \mathrm{O},-5.7550780742,-4.1659996796,-1.871640997$ $\mathrm{H}, 0,-2.3707765773,-1.7361821435,-2.4060484291$ $\mathrm{H}, \mathrm{O},-1.9401522837,-0.1330337752,-1.8229345732$ $\mathrm{H}, 0,-1.9966232219,-0.7353987694,1.9973984064$ $\mathrm{H}, \mathrm{O},-0.3747022811,-0.0981713269,2.2392254432$

\section{TS-4A}

Opt a $\mathrm{wB} 97 \mathrm{XD} / \mathrm{SDD} / 6-31 \mathrm{G}(\mathrm{d}, \mathrm{p})$ in toluene (SMD model) SCF Done: E (RwB97XD) $=-2529.95009238 \mathrm{a} \cdot \mathrm{u}$. Zero-point correction $=0.650991$ Hartree/Particle Sum of electronic and thermal Free Energies $=-2529.392571 \mathrm{a} \cdot \mathrm{u}$. @ $358.15 \mathrm{~K}$ Imaginary Freq. $=-19.4173 \mathrm{~cm}^{-1}$

Sp a B3LYP-D3 (BJ)/def2-TZVPP in toluene (SMD model) $\mathrm{SCF}$ Done: $\mathrm{E}(\mathrm{RB} 3 \mathrm{LYP})=-2531.58993329 \mathrm{a} \cdot \mathrm{u}$.

$\mathrm{Rh}, 0,1.3321231633,-0.4973506774,2.3827338173$ $0,0,3.3175320411,-0.7636063681,2.1007260029$ $C, 0,3.7649151132,-0.6989421865,0.8650232786$ $C, 0,3.4203332981,0.3252197576,-0.0230207746$ C, $0,4.6542994891,-1.7230532809,0.4023675141$ C, $0,3.8015885701,0.2405493391,-1.4011871934$ C, $0,5.1224133933,-1.7604087514,-0.8777336014$ C, $0,4.6860608731,-0.7955551464,-1.8213621415$ $\mathrm{H}, 0,5.8003582537,-2.5482288535,-1.190870556$ C, $0,4.601400773,-0.0004027538,-4.1135684208$ C, $0,3.6749831681,0.9902855965,-3.7191345134$ 
C, $0,3.2878612575,1.1080243977,-2.4076145462$

$\mathrm{C}, 0,5.0864234383,-0.8788117397,-3.1795599184$

$\mathrm{H}, 0,4.9086586409,-0.075840624,-5.1519559685$

$\mathrm{H}, 0,3.2540139389,1.6597848772,-4.4633890956$ $\mathrm{H}, 0,2.5551547052,1.8590674853,-2.1362319938$ $\mathrm{H}, 0,5.7755635902,-1.6679344363,-3.469280665$ $\mathrm{C}, 0,2.673305281,1.4716676492,0.5430333942$ C, $0,3.0049127999,2.7824952797,0.1652482425$ C, $0,1.6858978559,1.2870458944,1.5343655618$ $\mathrm{C}, 0,2.3600237348,3.8837705742,0.7159662855$ $\mathrm{H}, 0,3.8047568548,2.9423358454,-0.5511419053$ $\mathrm{C}, 0,1.0454087529,2.4038324357,2.0805609533$ C, $0,1.3724153351,3.6961966903,1.6754061866$ $\mathrm{H}, 0,2.6403563275,4.8847445263,0.4018849474$ $\mathrm{H}, 0,0.2826939623,2.264252329,2.8391089908$ $\mathrm{H}, 0,0.8574856749,4.5489287798,2.1095837207$ $\mathrm{C}, 0,-0.342878895,-1.6262359623,3.3790541666$ $\mathrm{C}, 0,0.5747135743,-2.5873612272,2.8880370091$ $\mathrm{C}, 0,0.7153309652,-2.3220741798,1.500477498$ C, $0,-0.1904861602,-1.2486489082,1.1220546934$ $\mathrm{C}, 0,-0.8841363911,-0.8385947569,2.3068066001$ $\mathrm{C}, 0,-0.4476048915,-0.776768064,-0.2823304767$ $\mathrm{C}, 0,-2.163691688,-0.0442578174,2.4255268141$ $\mathrm{H}, \mathrm{O},-0.6163684903,-1.4962569779,4.420113362$ $\mathrm{H}, 0,1.1393196599,-3.3019977455,3.4685046764$ $\mathrm{H}, 0,1.3871359767,-2.8220329382,0.814257299$ $0,0,-1.93569589,2.4741947056,3.4547573616$ $0,0,-1.019492394,-3.2061946067,-1.3758356066$ C, 0, $-2.3224151023,1.1700489738,1.5375040571$ C, $0,-2.1869700645,2.4696819547,2.1185960371$ C, $0,-2.3097676977,3.6033313563,1.3589514$ $\mathrm{C}, 0,-2.5593517759,3.5056847567,-0.0335894616$ $\mathrm{C}, 0,-2.6426029808,4.6643175036,-0.8484626428$ C, $0,-2.8797508222,4.5601760613,-2.1952950344$ C, $0,-3.0455214821,3.2898916119,-2.790238032$ C, $0,-2.9746184141,2.1529993056,-2.0249320873$ $\mathrm{C}, 0,-2.7315520553,2.2285661403,-0.6283923913$ $\mathrm{C}, 0,-2.6394462144,1.0613760818,0.1998087152$ C, $0,-1.786189098,3.7229090948,4.1003041809$ C, $0,-1.8621941138,-1.1430940914,-0.6597658552$ $C, 0,-2.9041166715,-0.2813116327,-0.4065936463$ $\mathrm{C}, 0,-4.2537851518,-0.6617967846,-0.714133558$ $C, 0,-5.3636424142,0.1851232927,-0.4579298815$ C, $0,-6.6400879936,-0.2104740336,-0.7718531014$ C, $0,-6.869778356,-1.4753959376,-1.356167796$ $C, 0,-5.8176561966,-2.3192912221,-1.6068090001$ C, $0,-4.4874391108,-1.9384660054,-1.2920982874$ C, $0,-3.3981058004,-2.8174113577,-1.5341071046$ C, $0,-2.1215072767,-2.434795146,-1.2128806925$ C, $0,-1.1823637955,-4.4841383131,-1.9562815448$ $\mathrm{H}, 0,-2.2010471149,4.5896109051,1.7946532121$ $\mathrm{H}, \mathrm{0},-2.508206385,5.6384377959,-0.3861243728$ $\mathrm{H}, \mathrm{0},-2.9363237916,5.4542413718,-2.8087010804$ $\mathrm{H}, \mathrm{O},-3.2268004864,3.2133348493,-3.8576497418$ $\mathrm{H}, \mathrm{O},-3.0991398092,1.1803364389,-2.4884239271$ $\mathrm{H}, 0,-0.9431154299,4.2902191276,3.6872539565$ $\mathrm{H}, \mathrm{O},-1.5863514779,3.4999878742,5.1487503465$ $\mathrm{H}, 0,-2.700443693,4.3240176583,4.0273746035$ 
$\mathrm{H}, \mathrm{O},-5.1939753937,1.1578662263,-0.0087874807$

$\mathrm{H}, 0,-7.4770158086,0.4507304162,-0.5704960465$

$\mathrm{H}, \mathrm{0},-7.8823929072,-1.7795546726,-1.6030884235$

$\mathrm{H}, \mathrm{O},-5.9889128445,-3.2962206892,-2.0508662835$

$\mathrm{H}, \mathrm{O},-3.6058603598,-3.7900992215,-1.9653436333$

$\mathrm{H}, \mathrm{O},-1.826516844,-5.1256425238,-1.3425124914$

$\mathrm{H}, \mathrm{O},-0.1855244548,-4.9232637938,-2.0073048508$

$\mathrm{H}, \mathrm{O},-1.5967759533,-4.4140865324,-2.9692487109$

$\mathrm{H}, 0,0.2731579037,-1.2410009088,-0.9564610645$

$\mathrm{H}, \mathrm{O},-0.3133406654,0.3036592931,-0.3391537412$

$\mathrm{H}, 0,-2.975268062,-0.7474682135,2.1991120867$

$\mathrm{H}, 0,-2.2879656778,0.2508648351,3.467857089$

$\mathrm{Cl}, 0,5.1339331814,-2.9691200061,1.5348502824$

\section{TS-4B}

Opt \& wB97XD/SDD/6-31G(d,p) in toluene (SMD model)

$\mathrm{SCF}$ Done: $\mathrm{E}(\mathrm{RwB} 97 \mathrm{XD})=-2529.94739942 \mathrm{a} \cdot \mathrm{u}$.

Zero-point correction $=0.650827$ Hartree/Particle

Sum of electronic and thermal Free Energies = -2529.390632 a.u. @ $358.15 \mathrm{~K}$ Imaginary Freq. $=-19.5521 \mathrm{~cm}^{-1}$

Sp a B3LYP-D3 (BJ)/def2-TZVPP in toluene (SMD model)

SCF Done: E(RB3LYP) $=-2531.58795943$ a.u.

$\mathrm{Rh}, 0,1.3080997874,-0.4610086262,-0.3134297762$

$0,0,2.9473813465,-1.0032542023,-1.3615990435$

$\mathrm{C}, 0,4.1350093462,-0.8356283518,-0.824876943$

$C, 0,4.535126092,0.3420328437,-0.1857625711$

C, $0,5.0802257072,-1.9100305492,-0.9169541901$

$\mathrm{C}, 0,5.8008985872,0.4052536909,0.4830780078$

$\mathrm{C}, 0,6.3265748384,-1.8353393466,-0.3713614304$

$\mathrm{C}, 0,6.7111872577,-0.6850671515,0.3645866486$

$\mathrm{H}, 0,7.0181466496,-2.6665314496,-0.4672776802$

$\mathrm{C}, 0,8.331076434,0.4533967831,1.7699115385$

C, $0,7.4144342473,1.514382665,1.9388379313$

C, 0,6.1907242205,1.491849529,1.3171985173

C, $0,7.9753669622,-0.6272440798,1.0047361559$

$\mathrm{H}, 0,9.2994937645,0.4869553359,2.2590542269$

$\mathrm{H}, 0,7.6756895611,2.3544615835,2.5756081098$

$\mathrm{H}, 0,5.4956254437,2.3062992678,1.4846600114$

$\mathrm{H}, 0,8.655656903,-1.4664678794,0.8849345537$

$\mathrm{C}, 0,3.6127351758,1.4948955592,-0.289640476$

$\mathrm{C}, 0,4.1233898905,2.7687008005,-0.5868139043$

$\mathrm{C}, 0,2.2109459628,1.3287550842,-0.2132184026$

$\mathrm{C}, 0,3.2866484927,3.8588342551,-0.7919073615$

$\mathrm{H}, 0,5.1950232284,2.895839651,-0.7047759215$

C, $0,1.3818702215,2.4426587338,-0.4052941263$

$\mathrm{C}, 0,1.9087829782,3.6973528913,-0.7052533318$

$\mathrm{H}, 0,3.7133002279,4.8259887205,-1.0407775469$

$\mathrm{H}, 0,0.305555101,2.3256037085,-0.3516315633$

$\mathrm{H}, \mathrm{0}, 1.2449940546,4.5376292629,-0.8884936268$

$\mathrm{Cl}, 0,4.5888524922,-3.3673181196,-1.7539349165$

$\mathrm{C}, 0,0.703160126,-0.9448749739,1.6677783432$

C, $0,1.2305012773,-2.1146473986,0.9956011294$

C, $0,0.3244372492,-2.4771292753,-0.0401019281$

$\mathrm{C}, 0,-0.6795493752,-1.4814327234,-0.0862873821$

$\mathrm{C}, 0,-0.4988954898,-0.5574802081,1.0205678558$

$\mathrm{C}, 0,-1.7830192165,-1.402728872,-1.1031482597$ 
C, $0,-1.510668529,0.3813505608,1.6382511725$

$\mathrm{H}, 0,1.1340586297,-0.4709659899,2.5395593229$

$\mathrm{H}, 0,2.146402357,-2.6299895069,1.2537496426$

$\mathrm{H}, 0,0.4467608082,-3.2832683693,-0.7489873452$

$0,0,-1.0184134807,3.0619569571,1.57984784$

$0,0,-2.7497655157,-3.9155537703,-0.759506262$

$C, 0,-2.2933301554,1.2897448009,0.7176537244$

C, $0,-1.9734724868,2.6858932229,0.6962351088$

$C, 0,-2.6272533842,3.5486659707,-0.1464123268$

C, $0,-3.6492174811,3.0703127939,-1.0069035274$

C, $0,-4.3185285032,3.9382485937,-1.9081538491$

$C, 0,-5.3171654554,3.4700073632,-2.7236084641$

C, $0,-5.6928613114,2.1091938882,-2.6762502748$

C, $0,-5.0647933014,1.2479864317,-1.8117494577$

C, $0,-4.0293642661,1.7032033004,-0.9541301574$

$C, 0,-3.3465889433,0.830013139,-0.0440565927$

C, $0,-0.6960027244,4.433345978,1.6791689178$

C, $0,-3.1052159585,-1.6277160156,-0.4094296705$

$C, 0,-3.8254599501,-0.5792794165,0.1119167167$

C, $0,-5.037030227,-0.823328257,0.8440788408$

C, $0,-5.7977573326,0.2208678421,1.4320822013$

C, $0,-6.9475636496,-0.051055134,2.1307605397$

C, $0,-7.3931142411,-1.3827816486,2.2781357395$

C, $0,-6.6744355604,-2.4137519242,1.7283718834$

C, $0,-5.479906741,-2.1637491113,1.0043954492$

C, $0,-4.7215756474,-3.2310598529,0.4540980307$

$C, 0,-3.5585616332,-2.9708093275,-0.2225875794$

C, $0,-3.1255201932,-5.2705425278,-0.617704515$

$\mathrm{H}, \mathrm{O},-2.3885530047,4.6058788586,-0.1648608024$

$\mathrm{H}, \mathrm{O},-4.0233743647,4.9833774073,-1.9429131831$

$\mathrm{H}, \mathrm{O},-5.8196394818,4.1443970496,-3.4102433534$

$\mathrm{H}, \mathrm{O},-6.4797406588,1.7441690794,-3.3285926924$

$\mathrm{H}, 0,-5.3534532518,0.2027560345,-1.7833270875$

$\mathrm{H}, 0,-0.3311535554,4.8349019674,0.72792921$

$\mathrm{H}, \mathrm{O}, 0.1014356471,4.5026146633,2.4190939698$

$\mathrm{H}, \mathrm{O},-1.5601852075,5.0202270943,2.014225827$

$\mathrm{H}, 0,-5.4614191991,1.2463461182,1.3255315121$

$\mathrm{H}, 0,-7.5164165174,0.7603635691,2.5735596516$

$\mathrm{H}, \mathrm{O},-8.3045147386,-1.5886130239,2.8311149907$

$\mathrm{H}, \mathrm{O},-7.0088419679,-3.4411899565,1.8435301903$

$\mathrm{H}, \mathrm{O},-5.0778384815,-4.2450054531,0.5963970274$

$\mathrm{H}, \mathrm{O},-3.1800664701,-5.562539578,0.4379283879$

$\mathrm{H}, 0,-2.3470999467,-5.8537983651,-1.1105342157$

$\mathrm{H}, \mathrm{O},-4.0880650691,-5.4729043156,-1.1027624631$

$\mathrm{H}, \mathrm{O},-1.616655214,-2.1540912484,-1.875806269$

$\mathrm{H}, \mathrm{O},-1.7751809442,-0.4185869023,-1.5782414148$

$\mathrm{H}, \mathrm{O},-2.2193106594,-0.2542370391,2.1838858871$

$\mathrm{H}, \mathrm{O},-0.9910608189,0.9856610943,2.3816620364$

\section{TS-5A}

Opt a wB97XD/SDD/6-31G $(\mathrm{d}, \mathrm{p})$ in toluene (SMD model)

$\mathrm{SCF}$ Done: $\mathrm{E}(\mathrm{RwB} 97 \mathrm{XD})=-2529.95029725 \mathrm{a} \cdot \mathrm{u}$.

Zero-point correction $=0.651589$ Hartree/Particle

Sum of electronic and thermal Free Energies $=-2529.390995 \mathrm{a} \cdot \mathrm{u}$. d $358.15 \mathrm{~K}$

Imaginary Freq. $=-117.3049 \mathrm{~cm}^{-1}$

Sp a B3LYP-D3 (BJ)/def2-TZVPP in toluene (SMD model)

SCF Done: E (RB3LYP) $=-2531.59270387 \mathrm{a} \cdot \mathrm{u}$. 
$\mathrm{Rh}, 0,-0.044979249,0.0557703388,-0.039546882$ $0,0,-0.0643268171,0.0649836369,1.91150063$ $C, 0,0.9234488597,-0.0081202253,2.7594783234$ C, 0,2.199071178,0.567152237,2.5918738955 $C, 0,0.6116343815,-0.7844001939,3.9338830108$ C, 0, 3.1353972283,0.4133529486,3.7159964711 C, $0,1.5443219951,-1.1040258306,4.8531104041$ C, $0,2.8340718506,-0.5213618497,4.7582415343$ $\mathrm{H}, 0,1.3142915638,-1.7753119512,5.6729641208$ C, $0,4.9881750842,-0.2166721346,5.8448440561$ $C, 0,5.2411838821,0.8238494177,4.9389052866$ C, $0,4.3490609342,1.1315317802,3.9351603251$ C, $0,3.7729170362,-0.8462461864,5.7662776037$ $\mathrm{H}, 0,5.7042242152,-0.4705525406,6.6195117689$ $\mathrm{H}, 0,6.1408201108,1.4237616215,5.0388107931$ $\mathrm{H}, 0,4.5812450772,2.0182748497,3.3760918256$ $\mathrm{H}, 0,3.4890197495,-1.5959722826,6.5002104511$ C, $0,2.5042586611,1.2033050467,1.2680651764$ C, $0,3.6745140415,1.9758556718,1.069220194$ C, $0,1.6977678643,1.0182266894,0.0971362262$ C, $0,4.0296282663,2.5644512952,-0.1307125046$ $\mathrm{H}, 0,4.3734711111,2.1267562173,1.8644712011$ C, $0,2.0855664393,1.6330570704,-1.1149148067$ C, $0,3.2210143991,2.4092380585,-1.2494896393$ $\mathrm{H}, 0,4.9459925954,3.1453262287,-0.1817011492$ $\mathrm{H}, \mathrm{O}, 1.456325978,1.5050118842,-1.988684038$ $\mathrm{H}, 0,3.471448179,2.8724068004,-2.1992488821$ $\mathrm{Cl}, 0,-1.0071561797,-1.414408473,4.1143154945$ C, $0,-0.0258535761,-0.6987274071,-2.0767266201$ C, $0,-1.1809837042,0.0879553841,-1.9066605524$ C, $0,-2.0417158478,-0.5734753512,-0.9392778755$ C, $0,-1.3903032267,-1.7080059407,-0.4618684376$ C, $0,-0.0785754833,-1.7680692162,-1.115045028$ C, $0,-1.8689429472,-2.623419192,0.6305441161$ C, $0,0.9311483947,-2.8822477728,-1.0346375559$ $\mathrm{H}, 0,0.7973168673,-0.5156202346,-2.7529654391$ $\mathrm{H}, 0,-1.4214604447,0.9965473157,-2.4426890508$ $\mathrm{H}, 0,-2.99968252,-0.2070944247,-0.5947795191$ $0,0,3.2030111322,-1.9372318329,0.1411642825$ $0,0,-3.9555508796,-3.8284388771,-0.6403871343$ C, $0,1.303990719,-3.2993873217,0.3696197272$ C, 0,2.4929129169,-2.7549649327,0.951562727 C, $0,2.8660589846,-3.0910633064,2.2279661849$ C, $0,2.0668006272,-3.9734567893,2.9971524226$ C, $0,2.421484554,-4.3065632621,4.3305089424$ C, $0,1.6348405526,-5.1444026735,5.0787054874$ C, $0,0.4548876322,-5.6917143605,4.5261811605$ C, $0,0.0931810377,-5.3946583487,3.2363805989$ C, $0,0.8881587668,-4.5319022328,2.4369398044$ $C, 0,0.538425907,-4.1904934495,1.0897956549$ C, $0,4.4498637535,-1.4567532949,0.6035026233$ C, $0,-1.7932103773,-4.0762649423,0.2259909217$ C, $0,-0.6571116798,-4.8203421716,0.4480737818$ C, $0,-0.5882485501,-6.1903291398,0.0249722571$ $C, 0,0.5746092327,-6.9855237652,0.2009444506$ C, $0,0.6026935342,-8.294642552,-0.210601706$ C, $0,-0.5310744711,-8.8729974173,-0.8223078484$ 
$C, 0,-1.6645486817,-8.1254379307,-1.0180353008$

$C, 0,-1.7213585234,-6.7680740119,-0.6077747398$

C, $0,-2.8824370429,-5.9818542126,-0.8332744806$

$\mathrm{C}, 0,-2.9101078362,-4.6679957383,-0.4416042383$

C, $0,-5.0808277482,-4.3244683093,-1.3348338245$

$\mathrm{H}, 0,3.7601906721,-2.6781920347,2.6799440877$

$\mathrm{H}, 0,3.3256459931,-3.8729325359,4.7494720557$

$\mathrm{H}, \mathrm{O}, 1.9150286573,-5.3878225422,6.0992977853$

$\mathrm{H}, 0,-0.1674405967,-6.3481376793,5.1261624579$

$\mathrm{H}, \mathrm{O},-0.815201323,-5.8151088356,2.817784022$

$\mathrm{H}, 0,4.3472972936,-0.8816508562,1.5285844952$

$\mathrm{H}, 0,4.8286049544,-0.8008781559,-0.180453836$

$\mathrm{H}, \mathrm{0}, 5.1526790494,-2.2844452274,0.7635178765$

$\mathrm{H}, 0,1.4494994309,-6.5474972279,0.6690279018$

$\mathrm{H}, \mathrm{O}, 1.4995335261,-8.8886852438,-0.0661872002$

$\mathrm{H}, \mathrm{0},-0.5015878128,-9.910438542,-1.1413297219$

$\mathrm{H}, \mathrm{0},-2.5372969558,-8.5632265343,-1.495205599$

$\mathrm{H}, \mathrm{O},-3.7304920009,-6.4416078026,-1.3281016797$

$\mathrm{H}, \mathrm{O},-4.8130023427,-4.6609212189,-2.3439201392$

$\mathrm{H}, \mathrm{O},-5.7821885793,-3.4926695316,-1.4098127817$

$\mathrm{H}, \mathrm{O},-5.5589337272,-5.1488971365,-0.7917607752$

$\mathrm{H}, \mathrm{O},-2.893934628,-2.3525719794,0.8873356542$

$\mathrm{H}, \mathrm{O},-1.2574288352,-2.457409418,1.5206371453$

$\mathrm{H}, 0,0.5101876031,-3.73825684,-1.5745030474$

$\mathrm{H}, 0,1.8267177097,-2.5646918011,-1.5696091965$

\section{TS-5B}

Opt @ wB97XD/SDD/6-31G(d,p) in toluene (SMD model)

$\mathrm{SCF}$ Done: $\mathrm{E}(\mathrm{RwB} 97 \mathrm{XD})=-2529.94433717 \mathrm{a} \cdot \mathrm{u}$.

Zero-point correction $=0.651068$ Hartree/Particle

Sum of electronic and thermal Free Energies = -2529.386511 a.u. @ $358.15 \mathrm{~K}$

Imaginary Freq. $=-197.5774 \mathrm{~cm}^{-1}$

$\mathrm{Sp}$ a B3LYP-D3 (BJ)/def2-TZVPP in toluene (SMD model)

SCF Done: E(RB3LYP $)=-2531.58703862 \mathrm{a} \cdot \mathrm{u}$.

$\mathrm{Rh}, 0,-0.7744481337,-2.3086739218,0.4108803137$

$0,0,-2.5509387078,-2.0400879819,-0.3280224603$

$\mathrm{C}, 0,-3.3764155586,-1.0342301771,-0.3213265485$

C, $0,-3.2795531415,0.136364122,0.468279966$

$\mathrm{C}, 0,-4.4561264234,-1.2109960538,-1.2602523848$

$\mathrm{C}, 0,-4.2928524491,1.1793143974,0.2186852755$

C, $0,-5.3863563135,-0.2619335228,-1.4827344811$

$\mathrm{C}, 0,-5.3125953586,0.9599337679,-0.7671212978$

$\mathrm{H}, 0,-6.1769121901,-0.4131564115,-2.2098926886$

$\mathrm{C}, 0,-6.313102545,3.1565583256,-0.4590505083$

$\mathrm{C}, 0,-5.3489173212,3.3972062886,0.5289941183$

$\mathrm{C}, 0,-4.3970163275,2.4555351978,0.8513561246$

$\mathrm{C}, 0,-6.2859746188,1.9386626422,-1.0833318859$

$\mathrm{H}, \mathrm{O},-7.0588878522,3.9032385133,-0.7106383611$

$\mathrm{H}, 0,-5.3450978499,4.3412912703,1.0657627562$

$\mathrm{H}, \mathrm{0},-3.7436386068,2.7512983812,1.6446781432$

$\mathrm{H}, 0,-7.0199868515,1.693110622,-1.8460412554$

$\mathrm{C}, 0,-2.2151310892,0.1563241934,1.525845195$

$C, 0,-2.0494867819,1.2411926059,2.4250698717$

$\mathrm{C}, 0,-1.3001408719,-0.9213638881,1.7438350007$

C, $0,-1.251267728,1.2068158684,3.5517600023$

$\mathrm{H}, 0,-2.5141551994,2.1858139765,2.242174521$ 
$C, 0,-0.5513831294,-0.9678689747,2.94121836$

$\mathrm{C}, 0,-0.54084342,0.0540981201,3.8689423454$

$\mathrm{H}, 0,-1.1928067705,2.0897320934,4.1808861455$

$\mathrm{H}, 0,0.0690235956,-1.838149366,3.1297426046$

$\mathrm{H}, 0,0.0461200736,-0.0204376882,4.7793950225$

C, $0,0.3918343575,-3.3779680357,-1.2140802849$

$\mathrm{C}, 0,0.3331752289,-4.2049769302,-0.0950632516$

C, $0,0.9511921576,-3.5068369921,1.0205516606$

C, $0,1.4075133321,-2.2488529798,0.574720847$

C, $0,0.9674194452,-2.1150813069,-0.8051666547$

$\mathrm{C}, 0,2.1776365445,-1.2403716458,1.389901654$

C, $0,1.2432881138,-0.9713137717,-1.7473688302$

$\mathrm{H}, \mathrm{O},-0.0385024274,-3.5730934078,-2.1869118487$

$\mathrm{H}, \mathrm{O},-0.1250221428,-5.1836353122,-0.043506147$

$\mathrm{H}, 0,1.0897163635,-3.9105216593,2.0150105372$

$0,0,-1.1000893962,0.2910424615,-2.3472628891$

$0,0,4.6785390961,-2.3549096759,1.5379054616$

C, $0,0.9227399576,0.3825405294,-1.1581276607$

C, $0,-0.3441475934,0.9757999162,-1.4567712488$

$\mathrm{C}, 0,-0.7168776397,2.1625927173,-0.8806408146$

C, $0,0.1430524083,2.8070318364,0.0437520022$

$\mathrm{C}, 0,-0.2557439852,3.9933140126,0.7124169305$

C, $0,0.5764013776,4.6045544372,1.6156670604$

C, $0,1.8521167974,4.0614012509,1.8871699259$

C, $0,2.2665268991,2.9184024001,1.2500590538$

C, $0,1.4246457967,2.2594146877,0.3176406207$

$\mathrm{C}, 0,1.8065829374,1.0418316418,-0.3325522931$

C, $0,-2.230469444,0.9421716932,-2.8952268496$

$\mathrm{C}, 0,3.3481313093,-0.642377268,0.6508640264$

$\mathrm{C}, 0,3.1766986058,0.4739372232,-0.1344519308$

C, $0,4.2904244615,1.0613919292,-0.8208608454$

C, $0,4.1589453153,2.2060048866,-1.6497266593$

$\mathrm{C}, 0,5.250086298,2.7454563642,-2.2840026944$

C, $0,6.5265211944,2.1620849522,-2.1244347589$

$\mathrm{C}, 0,6.6824557981,1.0488952937,-1.338035474$

C, $0,5.5730016509,0.4684013553,-0.670115939$

$\mathrm{C}, 0,5.7256554995,-0.6937818716,0.1316845762$

$\mathrm{C}, 0,4.6398696358,-1.2406187662,0.7667926199$

$\mathrm{C}, 0,5.9264958904,-2.9912949316,1.7213914291$

$\mathrm{H}, \mathrm{O},-1.6838602223,2.6071262134,-1.0864653799$

$\mathrm{H}, \mathrm{O},-1.2393131695,4.4041963378,0.4998649334$

$\mathrm{H}, 0,0.2567715171,5.5085350129,2.1252743777$

$\mathrm{H}, 0,2.5050491119,4.5500650071,2.6036087737$

$\mathrm{H}, 0,3.2452821257,2.5007858264,1.4635973481$

$\mathrm{H}, \mathrm{O},-2.9706206142,1.1907071717,-2.1303467384$

$\mathrm{H}, \mathrm{O},-2.6700353649,0.2394935955,-3.6033342114$

$\mathrm{H}, 0,-1.9338155698,1.8570771861,-3.4239899739$

$\mathrm{H}, 0,3.180481552,2.6569077196,-1.7760710366$

$\mathrm{H}, 0,5.1326129969,3.6232669105,-2.9115382266$

$\mathrm{H}, 0,7.3844118426,2.5960877879,-2.6290032954$

$\mathrm{H}, 0,7.6614796897,0.5929346278,-1.2171196066$

$\mathrm{H}, 0,6.7128832684,-1.1327869436,0.2199892877$

$\mathrm{H}, 0,6.338312967,-3.3497407776,0.770004874$

$\mathrm{H}, 0,5.738642348,-3.845220432,2.373187378$

$\mathrm{H}, 0,6.65162149,-2.3236873817,2.2027543291$

$\mathrm{H}, 0,2.5243364524,-1.7302421173,2.3010060773$

$\mathrm{H}, 0,1.5097917043,-0.4323935509,1.6927250877$

$\mathrm{H}, \mathrm{0}, 2.3015459212,-1.0208070407,-2.0258898181$ 
$\mathrm{H}, 0,0.6567503358,-1.127434072,-2.6529461119$

$\mathrm{Cl}, 0,-4.5260506555,-2.7012415153,-2.1709232932$

\section{INT-6- $\left(R_{\mathrm{a}}, R_{\mathrm{Rh}}\right)$}

Opt \& wB97XD/SDD/6-31G $(\mathrm{d}, \mathrm{p})$ in toluene (SMD model)

$\mathrm{SCF}$ Done: $\mathrm{E}(\mathrm{RwB} 97 \mathrm{XD})=-3069.27781813 \mathrm{a} \cdot \mathrm{u}$.

Zero-point correction $=0.847353$ Hartree/Particle

Sum of electronic and thermal Free Energies $=-3068.544281 \mathrm{a} . \mathrm{u}$. d $358.15 \mathrm{~K}$ Sp a B3LYP-D3 (BJ)/def2-TZVPP in toluene (SMD model)

SCF Done: $\mathrm{E}(\mathrm{RB} 3 \mathrm{LYP})=-3071.35097193 \mathrm{a} \cdot \mathrm{u}$.

$\mathrm{Rh}, 0,-1.3283669194,-1.3602389681,-0.2044287451$

$0,0,-0.9220039734,0.0335478522,1.2210123679$

$\mathrm{C}, 0,-0.9145484601,1.3347213548,1.1521730108$

$\mathrm{C}, 0,-1.6584597229,2.1044276686,0.2480702378$

$\mathrm{C}, 0,-0.1104518356,2.0098713394,2.1384115781$

C, $0,-1.4659409587,3.5404839363,0.2346466722$

$\mathrm{C}, 0,-0.033943648,3.3608773944,2.2238359252$

$\mathrm{C}, 0,-0.6918408366,4.1618361936,1.2601230468$

$\mathrm{H}, 0,0.5719097594,3.8309290607,2.9912293717$

$\mathrm{C}, 0,-1.0049943677,6.3738590053,0.3026857239$

$\mathrm{C}, 0,-1.7034575107,5.77430227,-0.7644723498$

C, $0,-1.9280970233,4.4188907682,-0.7924853114$

C, $0,-0.5030603011,5.5660895326,1.2891074063$

$\mathrm{H}, \mathrm{O},-0.8484524796,7.4475833574,0.3273794559$

$\mathrm{H}, \mathrm{O},-2.0680841985,6.3873946936,-1.583731503$

$\mathrm{H}, \mathrm{O},-2.4575447872,4.0190501799,-1.6448500773$

$\mathrm{H}, 0,0.0796898211,5.9862397105,2.1048091996$

$\mathrm{C}, 0,-2.5504447123,1.4157287701,-0.7265110169$

$\mathrm{C}, 0,-3.6743392786,2.1119534587,-1.2203392887$

$C, 0,-2.3442366065,0.1020978836,-1.220871532$

$\mathrm{C}, 0,-4.4045097647,1.6810950608,-2.3188395826$

$\mathrm{H}, 0,-3.9797726467,3.0250666806,-0.7223697583$

$\mathrm{C}, 0,-3.0552954944,-0.2911611667,-2.361978514$

C, $0,-4.0432207906,0.4953752322,-2.9451514015$

$\mathrm{H}, \mathrm{O},-5.2496799984,2.2648653614,-2.6711425889$

$\mathrm{H}, \mathrm{O},-2.8837677356,-1.2774184878,-2.7795869182$

$\mathrm{H}, \mathrm{O},-4.5745934865,0.1401478269,-3.8233579873$

$\mathrm{C}, 0,-0.593885822,-2.5427628758,-1.9108979305$

C, $0,-1.0179324213,-3.422141219,-0.8857412579$

$\mathrm{C}, 0,-0.1407903932,-3.2496739775,0.2446768108$

$\mathrm{C}, 0,0.7639306786,-2.2191777092,-0.0569905057$

C, $0,0.4919225787,-1.756501384,-1.4082583706$

C, $0,1.7568750213,-1.6175452747,0.8953118796$

C, $0,1.3942916756,-0.91041857,-2.2679672419$

$\mathrm{H}, \mathrm{O},-1.0073573739,-2.4664176233,-2.9068777808$

$\mathrm{H}, 0,-1.8312433813,-4.1321919166,-0.9510823734$

$\mathrm{H}, 0,-0.2022807686,-3.7688051851,1.1906352472$

$0,0,0.093537422,1.2913718187,-3.0975481276$

$0,0,3.4235499556,-3.7223560483,1.3332222099$

$\mathrm{C}, 0,1.7430486152,0.4338359744,-1.68322888$

C, $0,0.9942308287,1.5667869944,-2.1256448158$

$\mathrm{C}, 0,1.2366305401,2.813595721,-1.6112330595$

C, $0,2.2205931731,2.9862050254,-0.6040563446$

C, 0,2.4631366176,4.2613507302,-0.0310192032

C, 0,3.4014601018,4.4212157071,0.9561187324

C, $0,4.1460797442,3.3115820233,1.4153672725$ 
$C, 0,3.9463598535,2.0700726394,0.8658068848$ C, $0,2.9856418927,1.8747826955,-0.1601633379$ C, $0,2.7472979279,0.5927903905,-0.7548403202$ C, $0,-0.7052510322,2.3410806145,-3.5955543088$ C, $0,3.1610229165,-1.6135087849,0.3435956554$ $C, 0,3.6304003811,-0.5677791741,-0.4181788184$ $C, 0,4.9632219303,-0.5996535379,-0.9545649837$ C, $0,5.4789040821,0.4336387207,-1.7804384893$ C, $0,6.759126973,0.3727507979,-2.2720601814$ C, $0,7.5883504989,-0.7282660948,-1.9654844015$ C, $0,7.1138240064,-1.7485751267,-1.1808732103$ C, $0,5.7936740884,-1.714296277,-0.6612737891$ C, $0,5.2902817491,-2.7816068874,0.1285095511$ C, $0,4.0043404357,-2.739255203,0.6013203924$ C, $0,4.2025760111,-4.8539939624,1.6575010746$ $\mathrm{H}, 0,0.6677996633,3.6782469712,-1.9326043923$ $\mathrm{H}, 0,1.8713507733,5.1054268078,-0.3734023304$ $\mathrm{H}, 0,3.5698080529,5.4013756227,1.3925271087$ $\mathrm{H}, \mathrm{O}, 4.8793778972,3.4426393427,2.2050582101$ $\mathrm{H}, 0,4.5196408578,1.2201740034,1.221041156$ $\mathrm{H}, 0,-1.3046771396,2.7912651008,-2.8018773026$ $\mathrm{H}, 0,-1.3710115774,1.8921696249,-4.3329414211$ $\mathrm{H}, \mathrm{O},-0.0941383698,3.1145871972,-4.0788401023$ $\mathrm{H}, 0,4.8473159738,1.2811632632,-2.0227861522$ $\mathrm{H}, 0,7.1359805263,1.1738032963,-2.9002343366$ $\mathrm{H}, 0,8.6003721288,-0.7674533895,-2.3568852019$ $\mathrm{H}, 0,7.7436542734,-2.6031996817,-0.9481783122$ $\mathrm{H}, 0,5.9395194888,-3.6260833801,0.3298367004$ $\mathrm{H}, \mathrm{O}, 4.5284397511,-5.3914277356,0.7582694893$ $\mathrm{H}, 0,3.5587125973,-5.5063818969,2.2487294151$ $\mathrm{H}, \mathrm{O}, 5.0810438631,-4.5799912896,2.2545635249$ $\mathrm{H}, 0,1.7173537912,-2.1696419156,1.8350876361$ $\mathrm{H}, 0,1.4357657871,-0.5964848278,1.1133440898$ $\mathrm{H}, 0,2.3096129149,-1.4889272579,-2.4358996776$ $\mathrm{H}, 0,0.9133144534,-0.773968629,-3.2360828544$ $\mathrm{Cl}, 0,0.7826225651,1.0460537249,3.2961428106$ $C, 0,-3.467515337,-1.8297494212,0.5409266027$ C, $0,-2.7428431377,-1.9759339908,1.5300428827$ C, $0,-4.6850116591,-1.7593291401,-0.2305322831$ C, $0,-4.9726112259,-2.7300599453,-1.196310607$ C, $0,-5.5855221095,-0.7133345079,-0.0054466527$ C, $0,-6.1491462712,-2.6533412913,-1.93014386$ $\mathrm{H}, 0,-4.2665950037,-3.5356870552,-1.3715788516$ $C, 0,-6.7592462152,-0.6415189907,-0.7462860195$ $\mathrm{H}, \mathrm{O},-5.3477662699,0.0457015189,0.731605634$ $C, 0,-7.042249075,-1.606486313,-1.7093952493$ $\mathrm{H}, 0,-6.3679037491,-3.4091240656,-2.6779842$ $\mathrm{H}, 0,-7.4506811451,0.1774931323,-0.5761579072$ $\mathrm{H}, 0,-7.9580607256,-1.5441223784,-2.2889871501$ C, $0,-2.215185137,-2.3002046614,2.8291012451$ C, $0,-1.8942824412,-1.2925803223,3.7447581699$ C, $0,-2.0547856103,-3.6473082885,3.1891580031$ $\mathrm{C}, 0,-1.407463655,-1.6330752738,5.0018591827$ $\mathrm{H}, 0,-2.0131356208,-0.2571220471,3.4544545526$ C, $0,-1.5666050532,-3.976721666,4.4465425127$ $\mathrm{H}, \mathrm{O},-2.3179650298,-4.425187612,2.4785760274$ C, $0,-1.2388969151,-2.9694124866,5.3535668849$ $\mathrm{H}, 0,-1.1536299196,-0.8471453394,5.7057231935$ 
$\mathrm{H}, 0,-1.4447224394,-5.0197606534,4.7211591754$

$\mathrm{H}, \mathrm{O},-0.8548360947,-3.2280155092,6.3354744514$

\section{INT-6- $\left(S_{\mathrm{a}}, R_{\mathrm{Rh}}\right)$}

Opt \& wB97XD/SDD/6-31G $(\mathrm{d}, \mathrm{p})$ in toluene (SMD model)

$\mathrm{SCF}$ Done: $\mathrm{E}(\mathrm{RwB} 97 \mathrm{XD})=-3069.29156726 \mathrm{a} \cdot \mathrm{u}$.

Zero-point correction $=0.847891$ Hartree/Particle

Sum of electronic and thermal Free Energies = -3068.556915 a.u. @ $358.15 \mathrm{~K}$ $\mathrm{Sp}$ a B3LYP-D3 (BJ)/def2-TZVPP in toluene (SMD model)

SCF Done: $\mathrm{E}(\mathrm{RB} 3 \mathrm{LYP})=-3071.36399418 \mathrm{a} \cdot \mathrm{u}$.

$\mathrm{Rh}, 0,1.0805843443,1.0897949054,0.6252032744$

$0,0,2.1772204019,-0.2320328991,1.7690366877$

C, $0,3.241079063,-0.7964164589,1.2690041545$

C, $0,3.3344673271,-1.2086910632,-0.0654273216$

C, $0,4.3795103864,-1.0014624292,2.1202065493$

C, $0,4.6199872642,-1.512611403,-0.6211816012$

C, $0,5.5800873208,-1.4216304018,1.6349668624$

C, $0,5.7474932173,-1.6346365364,0.2423050189$

$\mathrm{H}, 0,6.4284397224,-1.5405293024,2.3013341291$

C, $0,7.2216512989,-2.0237761624,-1.6480290569$

C, $0,6.1233567335,-1.8476906416,-2.5175705355$

C, $0,4.8653703665,-1.6061314073,-2.0216091572$

C, 0, 7.029290967,-1.9108493056,-0.294551018

$\mathrm{H}, 0,8.2095965514,-2.2254177837,-2.0501748785$

$\mathrm{H}, 0,6.2747803367,-1.8936579569,-3.5921559545$

$\mathrm{H}, 0,4.0461783213,-1.4503838067,-2.714022565$

$\mathrm{H}, 0,7.8658458485,-2.0114836055,0.3924642173$

C, $0,2.0727815134,-1.3344028166,-0.8374955521$

C, $0,1.9433008168,-2.4215261229,-1.7229315205$

$C, 0,0.9643944966,-0.4765440356,-0.6696082451$

$\mathrm{C}, 0,0.7972135788,-2.6296242977,-2.4786501578$

$\mathrm{H}, 0,2.7633370696,-3.1277497693,-1.8063775753$

C, $0,-0.1865057644,-0.7048225157,-1.4310797717$

C, $0,-0.27679103,-1.7584089513,-2.3366718502$

$\mathrm{H}, 0,0.7427351027,-3.4731573375,-3.1602994949$

$\mathrm{H}, \mathrm{0},-1.0323888696,-0.0333959964,-1.3489741651$

$\mathrm{H}, 0,-1.1827035885,-1.8929014312,-2.9209179792$

$\mathrm{Cl}, 0,4.2110972496,-0.6564471875,3.8245534878$

C, $0,2.9674405321,1.8813857667,-0.1582351754$

C, $0,2.2121993731,1.8713584017,-1.1484434161$

C, $0,4.2194608388,2.0423205763,0.5460603143$

$C, 0,4.2557044608,2.4364255484,1.8864169338$

C, $0,5.4161775289,1.778962655,-0.1341493823$

C, $0,5.4747828223,2.5825175991,2.5358307537$

$\mathrm{H}, \mathrm{O}, 3.3244501442,2.599414894,2.4159380333$

$\mathrm{C}, 0,6.6306338102,1.9188410987,0.5245370838$

$\mathrm{H}, 0,5.3832565113,1.4398668392,-1.1641560783$

$\mathrm{C}, 0,6.6631445231,2.32092856,1.8583387395$

$\mathrm{H}, 0,5.4948879428,2.8787462055,3.5796472154$

$\mathrm{H}, 0,7.5524828346,1.694528858,-0.0024147083$

$\mathrm{H}, 0,7.6140669802,2.4197545277,2.3727873952$

C, $0,1.6983875067,2.1316247049,-2.4742716707$

C, $0,1.6490743519,1.1215092448,-3.4431965667$

C, $0,1.2708659947,3.4259876654,-2.8029684102$

C, $0,1.1699663792,1.4055882038,-4.7168003781$

$\mathrm{H}, 0,1.9793395088,0.120524507,-3.1900891531$ 
$C, 0,0.794063366,3.7011506067,-4.0791583856$ $\mathrm{H}, 0,1.3272334364,4.2119027373,-2.055893264$ C, $0,0.7391072036,2.6907311287,-5.0370385077$ $\mathrm{H}, \mathrm{O}, 1.1328177613,0.6160106421,-5.4605760748$ $\mathrm{H}, 0,0.4681211698,4.7064873561,-4.3267140853$ $\mathrm{H}, 0,0.36440066,2.9061186135,-6.0328595655$ C, $0,-1.0738788662,1.6422656207,0.7514704813$ $C, 0,-0.2950959336,2.7959944516,0.4719282368$ C, $0,0.5197600882,3.0677517018,1.6325723843$ C, $0,0.3217206184,2.0395786191,2.5598142876$ C, $0,-0.6630008126,1.1106586752,2.0036077358$ C, $0,1.0165720531,1.8783634534,3.8804800936$ C, $0,-1.3527886313,-0.025218992,2.7172692852$ $\mathrm{H}, 0,-1.832527264,1.2158881798,0.1111935826$ $\mathrm{H}, \mathrm{O},-0.3708712249,3.4120395156,-0.4134062108$ $\mathrm{H}, 0,1.2200347291,3.8853034561,1.7392246283$ $0,0,-0.461830884,-2.3748273884,1.6743612907$ $0,0,0.1313401658,4.1752599529,5.0514738166$ $C, 0,-0.4579400167,-0.939651231,3.5241550686$ C, $0,-0.0025725792,-2.1543737562,2.925269646$ $C, 0,0.8163783263,-3.0140439653,3.610975071$ C, $0,1.2474476165,-2.6969636081,4.9237904649$ C, $0,2.1276523845,-3.5527458779,5.6352399645$ C, $0,2.5631131491,-3.2260241108,6.8940477803$ C, $0,2.136848893,-2.0246777172,7.5023816326$ C, $0,1.2800615705,-1.1812022962,6.8400054538$ C, $0,0.8087778541,-1.495246433,5.5387463986$ $C, 0,-0.0728099658,-0.6324461411,4.8090818832$ C, $0,0.0332673743,-3.4967301417,0.9743888828$ C, $0,0.0046782653,1.8350356731,5.0024248601$ $C, 0,-0.5336539842,0.6442425307,5.433451464$ $C, 0,-1.5423731798,0.6250544332,6.452783358$ $\mathrm{C}, 0,-2.1435724174,-0.5783117905,6.9049417041$ C, $0,-3.1096962292,-0.5604047651,7.8798313349$ C, $0,-3.5228039079,0.664288371,8.4492847287$ C, $0,-2.9639706877,1.8442348524,8.0274299495$ C, $0,-1.9654855635,1.857025531,7.0191832438$ C, $0,-1.3971606983,3.0760172374,6.5627527718$ C, $0,-0.4486924711,3.0652560908,5.5723732455$ C, $0,-0.2509727144,5.4305456617,5.5731859697$ $\mathrm{H}, 0,1.1671717615,-3.9355052094,3.1615300745$ $\mathrm{H}, 0,2.4620866882,-4.4692899629,5.1569025809$ $\mathrm{H}, 0,3.2423649335,-3.8873139151,7.4236069105$ $\mathrm{H}, 0,2.4905653389,-1.7672753193,8.4959507138$ $\mathrm{H}, 0,0.9592489311,-0.2579188845,7.3108970814$ $\mathrm{H}, 0,1.1261362451,-3.4727784651,0.9092479532$ $\mathrm{H}, \mathrm{O},-0.385114408,-3.4333856629,-0.0295113854$ $\mathrm{H}, \mathrm{O},-0.2881938364,-4.4343535076,1.4467910693$ $\mathrm{H}, 0,-1.8280331106,-1.5205888725,6.4698252822$ $\mathrm{H}, \mathrm{O},-3.5589168172,-1.4900340766,8.214768063$ $\mathrm{H}, 0,-4.2871724762,0.6694541371,9.2204870258$ $\mathrm{H}, \mathrm{O},-3.282088842,2.7894538851,8.459393228$ $\mathrm{H}, 0,-1.7406282727,4.0031741845,7.0073734131$ $\mathrm{H}, 0,-1.320828159,5.6203349068,5.4234273086$ $\mathrm{H}, 0,0.3233639276,6.1760274617,5.0218255682$ $\mathrm{H}, \mathrm{O},-0.0132075996,5.5123439497,6.640837286$ $\mathrm{H}, 0,1.7027642652,2.7144838704,4.0260075101$ $\mathrm{H}, 0,1.6049483805,0.9555540535,3.8517208926$ 
$\mathrm{H}, 0,-2.1007973046,0.4208922168,3.3827357967$

$\mathrm{H}, 0,-1.8915579095,-0.6122436929,1.9721114213$

\section{INT-6- $\left(R_{\mathrm{a}}, S_{\mathrm{Rh}}\right)$}

Opt a wB97XD/SDD/6-31G(d,p) in toluene (SMD model)

$\mathrm{SCF}$ Done: $\mathrm{E}(\mathrm{RwB} 97 \mathrm{XD})=-3069.28513651 \mathrm{a} \cdot \mathrm{u}$.

Zero-point correction $=0.847391$ Hartree/Particle

Sum of electronic and thermal Free Energies $=-3068.553114 \mathrm{a} \cdot \mathrm{u}$. @ $358.15 \mathrm{~K}$

$\mathrm{Sp}$ a B3LYP-D3 (BJ)/def2-TZVPP in toluene (SMD model)

SCF Done: $\mathrm{E}(\mathrm{RB} 3 \mathrm{LYP})=-3071.35750484 \mathrm{a} \cdot \mathrm{u}$.

$\mathrm{Rh}, 0,0.9766937717,-1.7833928957,-1.13413364$

$0,0,2.1363876924,-1.6271787146,0.5660308355$

$C, 0,3.3705296594,-1.2229908035,0.5019702561$

C, $0,3.8046905169,-0.2141726801,-0.3623331512$

$C, 0,4.339253812,-1.8244676952,1.3738711937$

$C, 0,5.2070244098,-0.023492572,-0.5817241201$

C, $0,5.6730567726,-1.5773222067,1.2603812205$

$C, 0,6.1473872931,-0.7108285177,0.2414764955$

$\mathrm{H}, 0,6.3806057418,-2.0732263511,1.9171870685$

C, $0,8.0068774456,0.2283133875,-1.0075295041$

$\mathrm{C}, 0,7.0849187623,0.8762967077,-1.8585896908$

C, $0,5.7327184816,0.7589348517,-1.6505780081$

C, $0,7.5379982763,-0.5523865447,0.0182800292$ $\mathrm{H}, 0,9.0737811547,0.3362245168,-1.1760939679$ $\mathrm{H}, 0,7.4479932475,1.4676897374,-2.6942640653$ $\mathrm{H}, 0,5.048544964,1.2507063167,-2.3323981325$

$\mathrm{H}, 0,8.2301392919,-1.0797914534,0.6700053556$ $C, 0,2.7670670998,0.6407084032,-0.9881644324$ C, $0,3.0121175416,2.0254971096,-1.0671531036$ C, $0,1.5212421666,0.1618815241,-1.4434001707$ C, $0,2.1171612469,2.9046659152,-1.6620011898$ $\mathrm{H}, 0,3.9324901878,2.4148510333,-0.6418130811$ C, $0,0.6689208957,1.0465115116,-2.1043643127$ C, $0,0.9461227204,2.4049854419,-2.2177361913$ $\mathrm{H}, 0,2.3431420549,3.9661129589,-1.7007362842$ $\mathrm{H}, \mathrm{O},-0.2240415845,0.6698884697,-2.5838143957$ $\mathrm{H}, 0,0.243554305,3.0611664171,-2.7228932579$ C, $0,-0.2778369874,-3.2090281592,0.0574992197$ $C, 0,-0.2087302808,-3.7217052383,-1.2393557165$ $C, 0,-0.7788138182,-2.7284062325,-2.1166144102$ C, $0,-1.318020291,-1.6714185408,-1.3303920092$ $C, 0,-0.9243353035,-1.9066037075,0.0229412417$ C, $0,-2.2470293377,-0.6025751102,-1.8371351727$ C, $0,-1.3075398437,-1.1345799236,1.2614787618$ $\mathrm{H}, 0,0.149046172,-3.6529094859,0.9475208915$ $\mathrm{H}, 0,0.2566895765,-4.6480200713,-1.5463522047$ $\mathrm{H}, \mathrm{O},-0.903553087,-2.8226156746,-3.187094479$ $0,0,0.7396144861,0.4439873517,2.1387951603$ $0,0,-4.4031677293,-2.1611212114,-2.5294574293$ $C, 0,-1.3445384093,0.366525179,1.0773197111$ $C, 0,-0.208362336,1.138712667,1.481571174$ C, $0,-0.1444886091,2.4835951634,1.2185565209$ C, $0,-1.2224111591,3.1385105893,0.571814351$ $C, 0,-1.1587746921,4.5198974171,0.2535672742$ C, $0,-2.20836847,5.1495191208,-0.3661247461$ C, $0,-3.3755125651,4.4252784461,-0.6961998897$ 
C, $0,-3.4632247053,3.0864877305,-0.4041093051$ $C, 0,-2.3925341761,2.4077641731,0.2337818501$ C, $0,-2.4260632746,1.0009425765,0.5084732337$ C, $0,1.9392091873,1.1008550092,2.4855645126$ $C, 0,-3.5257242338,-0.5866688485,-1.0338666069$ $\mathrm{C}, 0,-3.6151638291,0.1924927507,0.096529676$ $\mathrm{C}, 0,-4.8149577717,0.2072360852,0.8788260469$ $\mathrm{C}, 0,-4.9469777776,0.9894961363,2.0552901688$ $\mathrm{C}, 0,-6.1151051032,0.9845224728,2.7759740424$ $\mathrm{C}, 0,-7.2076272971,0.1948312654,2.3540311863$ C, $0,-7.1061646235,-0.5769845883,1.2241635481$ $\mathrm{C}, 0,-5.911253697,-0.5931286306,0.4586891539$ C, $0,-5.7926933389,-1.4029445996,-0.7026390927$ C, $0,-4.6253377866,-1.4086817577,-1.4234318584$ $\mathrm{C}, 0,-5.4474583309,-2.999775988,-2.978862739$ $\mathrm{H}, 0,0.7290314785,3.0653339285,1.4878683241$ $\mathrm{H}, 0,-0.2563824682,5.069924972,0.5064596653$ $\mathrm{H}, \mathrm{O},-2.1437737899,6.2068210788,-0.6050040739$ $\mathrm{H}, \mathrm{O},-4.2028833707,4.9291740248,-1.186013187$ $\mathrm{H}, 0,-4.3589717265,2.5329312983,-0.6662419724$ $\mathrm{H}, 0,2.4379869251,1.5101221573,1.6011804707$ $\mathrm{H}, 0,2.5772091509,0.3438914467,2.9414430087$ $\mathrm{H}, 0,1.7572605917,1.9040357008,3.21196279$ $\mathrm{H}, 0,-4.1087013277,1.5963350896,2.3810223507$ $\mathrm{H}, \mathrm{O},-6.2015440979,1.5893317537,3.6730932398$ $\mathrm{H}, \mathrm{0},-8.1280247229,0.1970426697,2.930040634$ $\mathrm{H}, 0,-7.9424327303,-1.1907884285,0.9001462152$ $\mathrm{H}, \mathrm{O},-6.6400540298,-2.013643435,-0.9929142212$ $\mathrm{H}, \mathrm{O},-5.7189636785,-3.7443678731,-2.2205417596$ $\mathrm{H}, \mathrm{0},-5.0670501838,-3.5121018329,-3.8632625032$ $\mathrm{H}, \mathrm{O},-6.3373501576,-2.4207598701,-3.2545526413$ $\mathrm{H}, \mathrm{O},-2.4548110039,-0.7852023221,-2.8928565718$ $\mathrm{H}, 0,-1.7904216252,0.3817359683,-1.7498980569$ $\mathrm{H}, 0,-2.2928914202,-1.4907294385,1.5844618995$ $\mathrm{H}, 0,-0.5933296457,-1.387617599,2.045939282$ $\mathrm{Cl}, 0,3.7580980706,-2.9216027652,2.60780783$ $\mathrm{C}, 0,2.7153331558,-2.6192208543,-2.1588793886$ C, $0,2.1619041229,-1.9097104234,-3.0211521429$ C, $0,3.76257789,-3.4468095562,-1.6043420493$ C, $0,3.4842297465,-4.461345523,-0.6840544532$ $C, 0,5.0871090382,-3.2045323163,-1.9934385768$ $\mathrm{C}, 0,4.517486596,-5.2355718514,-0.1707903593$ $\mathrm{H}, 0,2.4627431586,-4.6155154915,-0.3548938803$ C, $0,6.115069129,-3.9764361822,-1.4684126562$ $\mathrm{H}, 0,5.3018263966,-2.3949992074,-2.6829392196$ $\mathrm{C}, 0,5.8328531243,-4.9936941674,-0.5588642934$ $\mathrm{H}, 0,4.2970124478,-6.0155192265,0.5507928822$ $\mathrm{H}, 0,7.1402084677,-3.7703861221,-1.7589241865$ $\mathrm{H}, 0,6.6392211691,-5.5902752556,-0.1438170766$ $\mathrm{C}, 0,1.8452241962,-1.3182427241,-4.3016489682$ C, $0,2.3051177204,-0.0370579267,-4.6307875364$ C, $0,1.0844909897,-2.0401376893,-5.2313727869$ C, $0,1.9998190547,0.5116243734,-5.8709374713$ $\mathrm{H}, \mathrm{O}, 2.8896752895,0.5207618284,-3.9080308792$ $\mathrm{C}, 0,0.7844336136,-1.4844911891,-6.469331563$ $\mathrm{H}, 0,0.7409625975,-3.0386146174,-4.9788715207$ $\mathrm{C}, 0,1.2373192393,-0.2060335581,-6.7891873638$ $\mathrm{H}, 0,2.3564591018,1.5065768545,-6.1173350368$ 
$\mathrm{H}, 0,0.1979741522,-2.0506762568,-7.1861029461$

$\mathrm{H}, 0,0.9989500494,0.2281850242,-7.7551330402$

\section{INT-6- $\left(S_{\mathrm{a}}, S_{\mathrm{Rh}}\right)$}

Opt \& wB97XD/SDD/6-31G $(\mathrm{d}, \mathrm{p})$ in toluene (SMD model)

$\mathrm{SCF}$ Done: $\mathrm{E}(\mathrm{RwB} 97 \mathrm{XD})=-3069.27712073 \mathrm{a} \cdot \mathrm{u}$.

Zero-point correction $=0.847042$ Hartree/Particle

Sum of electronic and thermal Free Energies = -3068.544971 a.u. @ $358.15 \mathrm{~K}$ $\mathrm{Sp}$ a B3LYP-D3 (BJ)/def2-TZVPP in toluene (SMD model)

SCF Done: $\mathrm{E}(\mathrm{RB} 3 \mathrm{LYP})=-3071.34955853 \mathrm{a} \cdot \mathrm{u}$.

$\mathrm{Rh}, 0,2.1478699513,-1.6203758994,-0.5755267636$

$0,0,1.5444565667,-0.2277075024,0.8184926392$

$C, 0,1.3264080325,1.0360957119,0.5927234137$

C, $0,1.8945019892,1.7838247245,-0.4467902851$

$\mathrm{C}, 0,0.4890862359,1.737425515,1.5312196826$

C, $0,1.3827051412,3.0998479538,-0.7255095004$

C, $0,0.1488570494,3.0455595045,1.388337327$

C, $0,0.5343281618,3.7469743544,0.2172675522$

$\mathrm{H}, \mathrm{O},-0.4717586221,3.5366645301,2.1314737253$

$\mathrm{C}, 0,0.3117426309,5.6929716394,-1.2187002058$

C, $0,1.098675289,5.037111945,-2.1896411849$

C, $0,1.611530667,3.7856320611,-1.9530568022$

C, $0,0.0300095546,5.0464410496,-0.0414096725$

$\mathrm{H}, \mathrm{O},-0.0809769252,6.6866664283,-1.4112289709$

$\mathrm{H}, 0,1.2793379253,5.5116740459,-3.1493825388$

$\mathrm{H}, 0,2.1671215006,3.2906442125,-2.7402603438$

$\mathrm{H}, \mathrm{O},-0.6008618826,5.5158305473,0.7097129805$

C, $0,3.0501498901,1.2321333372,-1.1976537887$

C, $0,4.0443679245,2.133510075,-1.638867506$

C, $0,3.2760429815,-0.1372374865,-1.4235451103$

$\mathrm{C}, 0,5.1262888767,1.7347219793,-2.4068564383$

$\mathrm{H}, 0,3.9608265954,3.1785478279,-1.3609413163$

C, $0,4.3553868457,-0.5223486164,-2.2302080919$

C, $0,5.2625645689,0.3929169116,-2.7463416934$

$\mathrm{H}, 0,5.8597653978,2.4676114713,-2.7295342077$

$\mathrm{H}, 0,4.5222324752,-1.5766956756,-2.4261643776$

$\mathrm{H}, 0,6.0926960035,0.0521563782,-3.3581160566$

$\mathrm{Cl}, 0,-0.0996105912,0.8595976137,2.9292317408$

C, $0,4.0293269796,-1.9203113663,0.6930909178$

C, $0,3.0634184721,-2.4237321806,1.2846980335$

C, $0,5.3637711083,-1.4325841955,0.4500962691$

C, $0,6.3228430679,-2.2697448078,-0.1299028214$

C, $0,5.6924604651,-0.1099121777,0.7690938543$

C, $0,7.5991678107,-1.7851989807,-0.3915173846$

$\mathrm{H}, 0,6.0584699984,-3.2923547475,-0.3816246936$

$\mathrm{C}, 0,6.9684928007,0.3668085061,0.5008301672$

$\mathrm{H}, 0,4.9376153875,0.5375587523,1.2007537587$

C, $0,7.9215982051,-0.4659234923,-0.0823632321$

$\mathrm{H}, \mathrm{O}, 8.3411875102,-2.4368362475,-0.842253285$

$\mathrm{H}, 0,7.2152097202,1.3967374479,0.7371066901$

$\mathrm{H}, 0,8.915731074,-0.0861277413,-0.2968927971$

C, $0,2.291570814,-3.1528604319,2.2649092254$

C, $0,1.0588274781,-2.6682478811,2.7185379543$

C, $0,2.7936466872,-4.36401398,2.7595070773$

C, $0,0.3412371269,-3.3926125024,3.6621809195$

$\mathrm{H}, 0,0.697505908,-1.7233781852,2.3259654663$ 
$C, 0,2.0658488668,-5.0805806434,3.7034206378$ $\mathrm{H}, 0,3.7503619035,-4.733273921,2.4030982622$ C, $0,0.8399018764,-4.5975998849,4.1553077919$ $\mathrm{H}, 0,-0.610813456,-3.0108159941,4.0178297689$ $\mathrm{H}, 0,2.4582530322,-6.017220088,4.086825831$ $\mathrm{H}, 0,0.2740592577,-5.1593567607,4.8921179239$ C, $0,0.3729151601,-3.0428127233,-0.7683108065$ C, $0,1.5812412408,-3.7452710277,-0.9354506445$ C, $0,2.2701737452,-3.2003524744,-2.071575461$ C, $0,1.4520809838,-2.1702128281,-2.6260119089$ $\mathrm{C}, 0,0.278681641,-2.0549868212,-1.8080314305$ $C, 0,1.7066222199,-1.4393547587,-3.9157651827$ C, $0,-1.0160536773,-1.3376946059,-2.0968416074$ $\mathrm{H}, \mathrm{O},-0.3577599157,-3.1977153868,0.0137771066$ $\mathrm{H}, \mathrm{O}, 1.9520018919,-4.5322370653,-0.2921639607$ $\mathrm{H}, \mathrm{O}, 3.2021554249,-3.556331555,-2.487579228$ $0,0,-1.7030370873,0.9428497047,-0.7603209619$ $0,0,1.9713268671,-3.6591609878,-5.4865541216$ C, $0,-0.9824849034,0.0017158525,-2.7956159164$ C, $0,-1.3517438652,1.1665854,-2.0510063436$ C, $0,-1.3914260222,2.4012879933,-2.6452838881$ C, $0,-1.0456954973,2.5501073061,-4.0100586343$ C, $0,-1.0364741213,3.8271371321,-4.6301174808$ C, $0,-0.6977105535,3.964163279,-5.9514269324$ $C, 0,-0.3520545593,2.8269844583,-6.7167371782$ C, $0,-0.3599081876,1.579942807,-6.1448451056$ $C, 0,-0.7062954428,1.4061406138,-4.7783053261$ C, $0,-0.7272036138,0.1201863912,-4.1465838889$ C, $0,-2.5432683703,1.8931166358,-0.1338795218$ C, $0,0.6318379884,-1.8273750284,-4.9001607486$ C, $0,-0.5339680317,-1.1036266561,-4.9845816708$ C, $0,-1.5904233469,-1.5248801498,-5.8619824639$ C, $0,-2.8250644707,-0.8315451977,-5.9605983581$ C, $0,-3.811719354,-1.2645592423,-6.8111667586$ C, $0,-3.6127958938,-2.4147309753,-7.6056214722$ C, $0,-2.4337745602,-3.111540357,-7.5241769045$ $C, 0,-1.398009668,-2.6905139718,-6.650596514$ $C, 0,-0.1858139076,-3.423899326,-6.546410227$ $C, 0,0.7953985325,-3.011869756,-5.6826819652$ C, $0,2.198986949,-4.8503260873,-6.2108055779$ $\mathrm{H}, \mathrm{O},-1.6545656014,3.2869399672,-2.0779084326$ $\mathrm{H}, 0,-1.2928166181,4.6951000107,-4.0294738772$ $\mathrm{H}, \mathrm{O},-0.6900298077,4.9470165968,-6.4129987795$ $\mathrm{H}, 0,-0.0776481657,2.9424068836,-7.7606867173$ $\mathrm{H}, \mathrm{O},-0.0897092317,0.7116487766,-6.7363803049$ $\mathrm{H}, 0,-2.0425344206,2.8534793757,0.0142332915$ $\mathrm{H}, 0,-2.7963923901,1.475627963,0.8414678905$ $\mathrm{H}, \mathrm{O},-3.4592265942,2.0460338997,-0.7188989713$ $\mathrm{H}, \mathrm{0},-2.9850383223,0.0524935544,-5.3530351232$ $\mathrm{H}, \mathrm{O},-4.7491053078,-0.7208733308,-6.8738562712$ $\mathrm{H}, \mathrm{O},-4.3972068483,-2.7485918169,-8.2780882903$ $\mathrm{H}, 0,-2.2770068628,-4.0016175674,-8.1277679192$ $\mathrm{H}, \mathrm{0},-0.0692696802,-4.3143031565,-7.1535040917$ $\mathrm{H}, 0,1.4437361814,-5.6114787875,-5.9789696438$ $\mathrm{H}, \mathrm{O}, 3.1794788344,-5.212620458,-5.8996445724$ $\mathrm{H}, 0,2.2112271821,-4.6678461121,-7.2922832205$ $\mathrm{H}, 0,2.6951672583,-1.7031947834,-4.2907229229$ $\mathrm{H}, 0,1.6932246075,-0.3621327211,-3.74807239$ 
$\mathrm{H}, 0,-1.5940732051,-2.037133555,-2.7164336596$

$\mathrm{H}, 0,-1.5553749704,-1.2309434549,-1.1554268121$

\section{TS-6- $\left(R_{\mathrm{a}}, R_{\mathrm{Rh}}\right)$}

Opt a wB97XD/SDD/6-31G(d,p) in toluene (SMD model)

$\mathrm{SCF}$ Done: $\mathrm{E}(\mathrm{RwB} 97 \mathrm{XD})=-3069.24749386 \mathrm{a} \cdot \mathrm{u}$.

Zero-point correction $=0.845126$ Hartree/Particle

Sum of electronic and thermal Free Energies $=-3068.517638 \mathrm{a} . \mathrm{u}$. A $358.15 \mathrm{~K}$ Imaginary Freq. $=-315.8845 \mathrm{~cm}^{-1}$

Sp a B3LYP-D3 (BJ)/def2-TZVPP in toluene (SMD model)

SCF Done: E (RB3LYP) $=-3071.32207911$ a.u.

$\mathrm{Rh}, 0,-0.1893760722,0.3365105909,3.396742947$

$0,0,-1.1416937103,-1.1138854464,4.4833004471$

$\mathrm{C}, 0,-0.6604924184,-2.1220863537,5.1277809733$

$C, 0,0.6951780286,-2.3614830579,5.4467138946$

$C, 0,-1.6487366818,-3.068159688,5.6038188083$

C, $0,1.0504515088,-3.6696588922,5.9724124154$

C, $0,-1.3294158202,-4.2027882176,6.2672205936$

C, $0,0.0360065237,-4.5554279514,6.436090281$

$\mathrm{H}, 0,-2.1056587304,-4.8752041895,6.6176936383$

C, $0,1.6558607375,-6.2689966109,7.021450904$

C, $0,2.663081948,-5.456170543,6.4689322649$

C, $0,2.3697060745,-4.2080473489,5.968558559$

C, $0,0.3599084058,-5.8193663015,6.9826362593$

$\mathrm{H}, 0,1.8958420789,-7.2442367577,7.4326556985$

$\mathrm{H}, 0,3.6862873365,-5.8177600424,6.4217103898$

$\mathrm{H}, 0,3.1774722608,-3.6454443773,5.521911281$

$\mathrm{H}, \mathrm{O},-0.4526259678,-6.4439061541,7.3447076548$

C, $0,1.705039716,-1.2943754415,5.2632808455$

C, $0,2.8877280304,-1.3359583205,6.0285726553$

C, $0,1.5231166286,-0.1261792662,4.4499015976$

$C, 0,3.9355613126,-0.4430905688,5.848959863$

$\mathrm{H}, 0,2.9838136376,-2.0718195241,6.8160005184$

C, $0,2.6162466761,0.7569360227,4.2784554884$

C, $0,3.8373995826,0.5780893044,4.9072343436$

$\mathrm{H}, 0,4.8232977334,-0.5380919008,6.4673551814$

$\mathrm{H}, 0,2.4648698106,1.6364498423,3.6588462109$

$\mathrm{H}, 0,4.6571622649,1.2702521034,4.7424557815$

C, $0,-0.4961586383,1.4457797977,5.0768336155$

C, $0,0.5124123245,1.2169208038,5.8318235695$

$\mathrm{Cl}, 0,-3.3330049384,-2.6964162907,5.3094129706$

$C, 0,-1.722329699,2.2131577142,5.3577921578$

C, $0,-1.6651103978,3.5452627851,5.777329671$

C, $0,-2.9662324066,1.5879245724,5.2050736257$

$C, 0,-2.8412199539,4.2464333168,6.0332199638$

$\mathrm{H}, \mathrm{O},-0.6999338118,4.0283287724,5.8980341054$

C, $0,-4.1342427195,2.2899830328,5.4760526186$

$\mathrm{H}, \mathrm{O},-2.9953600325,0.5496681312,4.8881929162$

$C, 0,-4.0764433941,3.6219904231,5.8847339205$

$\mathrm{H}, 0,-2.7898356842,5.2824245167,6.3542536459$

$\mathrm{H}, 0,-5.0938781436,1.794432788,5.3646061432$

$\mathrm{H}, 0,-4.9918646071,4.1688176539,6.0885894858$

C, $0,1.2166285345,1.1958666276,7.0773677266$

C, 0,2.2496152802,2.1061245029,7.3417152031

$C, 0,0.8348033933,0.2662391327,8.0606128281$

C, $0,2.8776189746,2.101119274,8.5811553817$ 
$\mathrm{H}, \mathrm{0}, 2.548119671,2.8077931113,6.5704044209$ C, 0, 1.4650499263,0.26967777029,9.2957443506 $\mathrm{H}, \mathrm{O}, 0.0497173566,-0.4482407996,7.835238408$ C, $0,2.486592754,1.1845426663,9.5543306197$ $\mathrm{H}, 0,3.674802284,2.8081429353,8.7863812337$ $\mathrm{H}, 0,1.1659614252,-0.4432776905,10.0568887224$ $\mathrm{H}, 0,2.9807994522,1.1808057895,10.5210495225$ C, $0,0.5701982732,1.0246706985,1.4068315672$ C, $0,-0.3788750943,1.9509004048,1.936103869$ C, $0,-1.6502271345,1.2918759641,1.9825748302$ C, $0,-1.4606797414,-0.0461840028,1.5545303724$ $C, 0,-0.0852041036,-0.208053769,1.1429540514$ C, $0,-2.5085675346,-1.1257646269,1.634985175$ C, $0,0.5041179464,-1.3669352497,0.3829927711$ $\mathrm{H}, \mathrm{O}, 1.6160452292,1.2257767698,1.2135423252$ $\mathrm{H}, \mathrm{O},-0.185099143,2.98171641,2.2012050429$ $\mathrm{H}, \mathrm{O},-2.5804071443,1.715358172,2.3349204774$ $0,0,2.5539901313,-2.2986905168,1.8708901571$ $0,0,-4.3442770257,-0.4233734901,-0.2538125286$ C, $0,0.3679016308,-2.6716275955,1.1235329418$ C, $0,1.462353433,-3.1011967607,1.9349895823$ C, $0,1.3790100083,-4.2498865322,2.6745484741$ C, $0,0.1725246728,-4.9980803322,2.6917137521$ $C, 0,0.0396724105,-6.1493491333,3.5088594328$ C, $0,-1.1391008211,-6.850058417,3.547836699$ C, $0,-2.2382362298,-6.4357175147,2.7636541048$ C, $0,-2.1316278599,-5.3348465457,1.9501318008$ $C, 0,-0.92707094,-4.5886080212,1.8915601346$ C, $0,-0.7847630229,-3.4212535706,1.0713672654$ $C, 0,3.6906720446,-2.6517723921,2.6258535681$ c, $0,-2.6607664662,-1.9318420366,0.3685386414$ C, $0,-1.8703097741,-3.0306572437,0.1180412296$ $C, 0,-2.0335670353,-3.7862680723,-1.0924030866$ C, $0,-1.2182085668,-4.9039593657,-1.4107983409$ C, $0,-1.4064656271,-5.6083387508,-2.5736845912$ C, $0,-2.4187376946,-5.2272329182,-3.4814619398$ C, $0,-3.2146546934,-4.1439309692,-3.2072211136$ $C, 0,-3.0407079467,-3.3949200784,-2.0145413101$ $C, 0,-3.8447194752,-2.257343257,-1.7391216972$ C, $0,-3.6476687649,-1.5374589088,-0.5889112083$ C, $0,-5.3677639403,0.0073806345,-1.1249053764$ $\mathrm{H}, 0,2.2005881272,-4.5845049822,3.296088883$ $\mathrm{H}, 0,0.8816620142,-6.4473768689,4.1276389044$ $\mathrm{H}, \mathrm{O},-1.2332577975,-7.7193258043,4.1919940697$ $\mathrm{H}, \mathrm{O},-3.1717184295,-6.9882667392,2.8077321883$ $\mathrm{H}, \mathrm{O},-2.9791498978,-5.0179393148,1.3508964928$ $\mathrm{H}, 0,3.4701749318,-2.6520884149,3.6968369917$ $\mathrm{H}, 0,4.4411826473,-1.8877936611,2.4203409298$ $\mathrm{H}, 0,4.0830711162,-3.6332409909,2.329772285$ $\mathrm{H}, \mathrm{O},-0.437815284,-5.2014587897,-0.7193362146$ $\mathrm{H}, \mathrm{O},-0.7735518985,-6.4611687043,-2.7987119995$ $\mathrm{H}, 0,-2.5632707722,-5.7914780262,-4.3978426192$ $\mathrm{H}, \mathrm{O},-3.9905021124,-3.8409835148,-3.9054832147$ $\mathrm{H}, \mathrm{O},-4.5988556789,-1.9719120978,-2.4638244417$ $\mathrm{H}, \mathrm{O},-4.9723208388,0.2739224576,-2.1130152272$ $\mathrm{H}, \mathrm{O},-5.803342359,0.8956858837,-0.6654988248$ $\mathrm{H}, \mathrm{O},-6.1465276284,-0.7568710132,-1.2391274061$ $\mathrm{H}, \mathrm{O},-3.4612236077,-0.6631386688,1.8963990817$ 
$\mathrm{H}, \mathrm{O},-2.2420250764,-1.7907382637,2.4628645229$

$\mathrm{H}, 0,0.0060818441,-1.4360942762,-0.589604071$

$\mathrm{H}, 0,1.5575229259,-1.1573009216,0.1962000044$

\section{TS-6- $\left(S_{\mathrm{a}}, R_{\mathrm{Rh}}\right)$}

Opt a wB97XD/SDD/6-31G (d,p) in toluene (SMD model)

$\mathrm{SCF}$ Done: $\mathrm{E}(\mathrm{RwB}$ 97XD) $=-3069.27474972 \mathrm{a} \cdot \mathrm{u}$.

Zero-point correction $=0.846533$ Hartree/Particle

Sum of electronic and thermal Free Energies = -3068.542370 a.u. @ $358.15 \mathrm{~K}$

Imaginary Freq. $=-304.0679 \mathrm{~cm}^{-1}$

$\mathrm{Sp}$ @ B3LYP-D3 (BJ)/def2-TZVPP in toluene (SMD model)

$\mathrm{SCF}$ Done: $\mathrm{E}(\mathrm{RB} 3 \mathrm{LYP})=-3071.34602899 \mathrm{a} \cdot \mathrm{u}$.

$\mathrm{Rh}, 0,-0.7790364486,-1.3041476726,-0.3218064767$

$0,0,-0.332501977,0.7000809626,-0.0326640658$

C, $0,-1.2712841956,1.5470061547,0.2712053095$

C, $0,-2.5277766823,1.5701580361,-0.3498008902$

$\mathrm{C}, 0,-1.012872882,2.5294127,1.2910518874$

$\mathrm{C}, 0,-3.5741437274,2.4088485938,0.1572897413$

$\mathrm{C}, 0,-1.9674547096,3.3942059396,1.7277776441$

$C, 0,-3.2838633055,3.3376235073,1.1969835322$

$\mathrm{H}, 0,-1.73880835,4.1098376049,2.5113305994$

C, $0,-5.5939523588,4.0831530294,1.244539885$

C, $0,-5.9029928121,3.1323242669,0.2492415434$

C, $0,-4.9264624397,2.321622189,-0.2769634379$

$\mathrm{C}, 0,-4.30614449,4.1722965932,1.7105008875$

$\mathrm{H}, \mathrm{0},-6.3720326012,4.7232212412,1.6483852447$

$\mathrm{H}, \mathrm{O},-6.9269473347,3.0315684962,-0.0989160001$

$\mathrm{H}, \mathrm{O},-5.1978530661,1.5848826125,-1.0243649421$

$\mathrm{H}, \mathrm{O},-4.0518795736,4.8791174908,2.4962483415$

$\mathrm{C}, 0,-2.7197589865,0.7217641475,-1.5444093694$

$\mathrm{C}, 0,-3.3231618237,1.273563011,-2.6883497516$

C, $0,-2.2983588999,-0.6273061643,-1.6046732436$

$\mathrm{C}, 0,-3.528136964,0.5338294068,-3.8440192168$

$\mathrm{H}, \mathrm{O},-3.6211535938,2.3174287268,-2.6636126622$

C, $0,-2.5204091557,-1.3678243873,-2.7793466219$

$\mathrm{C}, 0,-3.1205869811,-0.7995736764,-3.8916156224$

$\mathrm{H}, \mathrm{O},-3.9987036929,0.9971607592,-4.7058260884$

$\mathrm{H}, \mathrm{O},-2.2447712394,-2.4168211687,-2.8021488598$

$\mathrm{H}, \mathrm{O},-3.2803184174,-1.3944650585,-4.7858875991$

$\mathrm{C}, 0,-2.2714418237,-1.0843628817,1.0766047354$

$\mathrm{C}, 0,-2.9251379855,-1.4912220452,0.0474272992$

$\mathrm{C}, 0,0.325755907,-2.8209216285,-1.5831400134$

$C, 0,-0.2775539723,-3.4283926297,-0.4306728285$

$\mathrm{C}, 0,0.385481659,-2.9077959569,0.7287027958$

C, $0,1.2794484406,-1.9003184573,0.3089428398$

C, $0,1.2645867036,-1.867126631,-1.1543055394$

$\mathrm{C}, 0,2.1253766172,-1.0259446001,1.1929377588$

C, $0,2.1984814265,-1.1016233048,-2.0564545262$

$\mathrm{H}, \mathrm{O}, 0.069083584,-3.0219169948,-2.614688346$

$\mathrm{H}, \mathrm{O},-1.0227402945,-4.2124204675,-0.4430943828$

$\mathrm{H}, 0,0.1753337204,-3.1703248862,1.7570148276$

$\mathrm{Cl}, 0,0.5805527247,2.5734866342,2.0064066388$

$\mathrm{C}, 0,-2.3002647171,-0.687537773,2.4613635327$

C, $0,-1.1175350168,-0.4916180203,3.1838774599$

C, $0,-3.5351235848,-0.4464323521,3.0867072997$

C, $0,-1.1659552135,-0.0827824505,4.511353872$ 
$\mathrm{H}, \mathrm{O},-0.1671025821,-0.6322460953,2.6825445948$ C, $0,-3.5753590679,-0.0367041598,4.4119716036$ $\mathrm{H}, \mathrm{O},-4.4523719597,-0.5691717623,2.5193089931$ C, $0,-2.3926962144,0.1440595111,5.1293014366$ $\mathrm{H}, \mathrm{O},-0.2414186353,0.0765443304,5.0575623406$ $\mathrm{H}, \mathrm{O},-4.5335736,0.1514511079,4.8864337322$ $\mathrm{H}, 0,-2.4292471791,0.4714663324,6.1637262776$ C, $0,-4.0729720288,-2.3437831054,-0.2811279848$ C, $0,-4.1741461379,-3.5969359685,0.3355607761$ C, $0,-5.1019739628,-1.9093726452,-1.1234153548$ C, $0,-5.2815195875,-4.4064653796,0.1041271869$ $\mathrm{H}, \mathrm{O},-3.3799300176,-3.9257213992,0.9991976109$ C, $0,-6.210401831,-2.7178338165,-1.3452654156$ $\mathrm{H}, 0,-5.030979977,-0.9355038416,-1.5959399186$ C, $0,-6.3008589342,-3.9686592375,-0.737474904$ $\mathrm{H}, \mathrm{O},-5.3489491631,-5.3777852252,0.5842479211$ $\mathrm{H}, \mathrm{0},-7.0075311202,-2.3690552694,-1.9943352258$ $\mathrm{H}, \mathrm{O},-7.1662890411,-4.5988854188,-0.9177596125$ $0,0,0.7390428673,0.8626378344,-3.2402535014$ $0,0,3.6736574172,-2.9529859796,2.3408061705$ C, $0,2.4148350937,0.3486915695,-1.6873426555$ C, $0,1.6129732116,1.3426542764,-2.3260650527$ C, $0,1.7643537043,2.6710620644,-2.0235116713$ C, $0,2.7023824914,3.0770809657,-1.0408679037$ C, $0,2.8431717159,4.4421953338,-0.6801592491$ $C, 0,3.7287374363,4.8256768153,0.2942486487$ $\mathrm{C}, 0,4.518084059,3.8585786698,0.9550316223$ C, $0,4.4091645828,2.5316607166,0.6202424778$ C, $0,3.5055405209,2.1057966435,-0.3875111968$ C, $0,3.3500865492,0.728395663,-0.751841922$ C, $0,-0.1408172351,1.7794616307,-3.853628686$ C, $0,3.591834682,-1.1462180089,0.8503154713$ $\mathrm{C}, 0,4.1792987191,-0.3160516811,-0.0770588285$ C, $0,5.5630322119,-0.4703220536,-0.42217101$ C, $0,6.2006753183,0.3461006436,-1.3922978808$ C, $0,7.5286697203,0.1749638807,-1.6945943918$ C, $0,8.2835677574,-0.8247689988,-1.0423021869$ $C, 0,7.6914044007,-1.6361088968,-0.1077462022$ C, $0,6.3197854531,-1.4844653315,0.223368935$ C, $0,5.6949983314,-2.3343276159,1.17439563$ C, $0,4.3646961535,-2.1775042247,1.4685332541$ C, $0,4.3693857023,-3.9895046156,3.0004922038$ $\mathrm{H}, 0,1.1579557902,3.4310873964,-2.5020805537$ $\mathrm{H}, \mathrm{O}, 2.2233463474,5.1800321223,-1.1825292673$ $\mathrm{H}, 0,3.8194780932,5.8731455573,0.5657183633$ $\mathrm{H}, 0,5.210673084,4.1666605642,1.7321700271$ $\mathrm{H}, 0,5.0142276327,1.7928871218,1.1351138663$ $\mathrm{H}, \mathrm{O},-0.7418654138,2.3115522457,-3.1087988516$ $\mathrm{H}, 0,-0.8010590594,1.1876729729,-4.4871782915$ $\mathrm{H}, \mathrm{O}, 0.4052472075,2.5038591272,-4.4723532905$ $\mathrm{H}, 0,5.6241408231,1.115123493,-1.895183339$ $\mathrm{H}, 0,8.0015679982,0.8095992946,-2.4374889721$ $\mathrm{H}, 0,9.3341367442,-0.9517168182,-1.2856861299$ $\mathrm{H}, 0,8.2664348619,-2.41070374,0.3927942002$ $\mathrm{H}, 0,6.2915520891,-3.1073037494,1.6453334314$ $\mathrm{H}, 0,4.7711479637,-4.7207739626,2.2883459421$ $\mathrm{H}, 0,3.640737898,-4.4823966645,3.6452446131$ $\mathrm{H}, \mathrm{O}, 5.1864333994,-3.5967391543,3.6180453375$ 
$\mathrm{H}, 0,1.9630622873,-1.3149952012,2.232896071$

$\mathrm{H}, 0,1.7908941632,0.0093234593,1.0720071512$

$\mathrm{H}, 0,3.1625661426,-1.6223247703,-2.0413377416$

$\mathrm{H}, \mathrm{O}, 1.8126940344,-1.1643831871,-3.0748041946$

\section{TS-6- $\left(R_{\mathrm{a}}, S_{\mathrm{Rh}}\right)$}

Opt a wB97XD/SDD/6-31G $(\mathrm{d}, \mathrm{p})$ in toluene (SMD model)

SCF Done: $\mathrm{E}(\mathrm{RwB} 97 \mathrm{XD})=-3069.27224360 \mathrm{a} \cdot \mathrm{u}$.

Zero-point correction $=0.846756$ Hartree/Particle

Sum of electronic and thermal Free Energies $=-3068.538692 \mathrm{a} . \mathrm{u}$. @ $358.15 \mathrm{~K}$

Imaginary Freq. $=-290.9272 \mathrm{~cm}^{-1}$

Sp \& B3LYP-D3 (BJ)/def2-TZVPP in toluene (SMD model)

SCF Done: E (RB3LYP) $=-3071.34245201$ a.u.

$\mathrm{Rh}, 0,1.9006595935,-0.2509455691,3.4469411768$

$0,0,0.5012013496,-1.3448394447,2.3642186829$

C, $0,-0.7402793907,-1.3596723048,2.7454761647$

C, $0,-1.4439941198,-0.205640188,3.1064395929$

C, $0,-1.4468971938,-2.6111500434,2.7848089796$

$C, 0,-2.7511366584,-0.2988407191,3.6808852958$

C, $0,-2.7133839116,-2.718604927,3.2694910127$

C, $0,-3.3889967448,-1.5704170233,3.7626292659$

$\mathrm{H}, 0,-3.2037761126,-3.6860090208,3.3124466207$

C, $0,-5.3122589412,-0.5866758267,4.8706506098$

$\mathrm{C}, 0,-4.6742642865,0.6716616276,4.8258712252$

$C, 0,-3.4353831969,0.8122606486,4.249404443$

C, $0,-4.672851144,-1.6836697392,4.3501895852$

$\mathrm{H}, 0,-6.2934313536,-0.6842327547,5.3245288794$

$\mathrm{H}, \mathrm{O},-5.164467863,1.5386016204,5.2594159846$

$\mathrm{H}, \mathrm{O},-2.9606296386,1.7868624657,4.2417555984$

$\mathrm{H}, \mathrm{O},-5.1391190353,-2.6647382321,4.3918647228$

C, $0,-0.7760086328,1.092444487,2.8739918246$

C, $0,-1.4701066527,2.1124859637,2.2013469476$

C, $0,0.554251712,1.3520947263,3.2751871176$

C, $0,-0.9030782248,3.3571729706,1.9640760061$ $\mathrm{H}, \mathrm{O},-2.4778026599,1.9084143547,1.8508223582$ C, $0,1.0851763517,2.6367404635,3.0947135222$

C, $0,0.381009123,3.6288455389,2.4303170455$

$\mathrm{H}, \mathrm{O},-1.4643719358,4.1153687841,1.4269973505$

$\mathrm{H}, 0,2.0597962089,2.8665626224,3.5085790161$

$\mathrm{H}, 0,0.8325017784,4.6036882421,2.2735866552$

C, $0,0.7540641686,-0.6604846006,5.1207597636$

C, $0,0.8025242329,0.6211859305,5.1162975727$

$0,0,0.8592329287,-0.5865526152,-0.4227501048$

$0,0,6.950682251,2.1199756108,2.9009768067$

C, 0,3.9649790707,0.1559211911, 4.1027906474

C, $0,3.7984476826,-1.2673889139,4.0837189113$

C, $0,3.4916698656,-1.6247007972,2.7584948402$

C, $0,3.5742229337,-0.4421449288,1.9131754394$

C, $0,3.920662154,0.6440832159,2.7516862612$

C, $0,3.4639597338,-0.4740625389,0.4064500035$

C, $0,2.7762647534,0.7360255927,-0.1881002135$

$\mathrm{C}, 0,1.3838187636,0.6500691742,-0.5114148091$

$C, 0,0.6833217588,1.7642364696,-0.9007863688$

C, $0,1.3397556078,3.0148391853,-1.0258137086$

C, $0,0.6269976695,4.1897348567,-1.3793058101$

C, 0,1.2695148141,5.3972113555,-1.491080485 
$C, 0,2.6602424485,5.4856979544,-1.2596266211$ $C, 0,3.3763031068,4.3637553394,-0.9220341115$ C, $0,2.737894324,3.1030753574,-0.7953456793$ C, $0,3.4413141732,1.9249451934,-0.3811215467$ C, $0,-0.5414418295,-0.7303444187,-0.5179891493$ C, $0,4.2581448975,2.0519883754,2.3409459331$ C, $0,5.2951283178,2.0633984848,1.2447789975$ C, $0,4.9009634674,2.0050560656,-0.07094382$ C, $0,5.8701049202,1.9878755472,-1.1248201358$ C, $0,5.5043514617,1.909853446,-2.4934499886$ C, $0,6.4615276668,1.8969741761,-3.4772696311$ $C, 0,7.8312522252,1.9601752529,-3.1376634696$ C, $0,8.2142754053,2.0298657733,-1.821843656$ C, $0,7.2480472097,2.043824819,-0.7825990919$ C, $0,7.635761184,2.0978791432,0.583360027$ C, $0,6.6842351688,2.0945120278,1.571761082$ $\mathrm{C}, 0,8.3030309924,2.1872146211,3.3035070399$ $\mathrm{H}, 0,4.2206799302,0.7577707729,4.965247822$ $\mathrm{H}, 0,3.8143333668,-1.9232977636,4.9429681752$ $\mathrm{H}, 0,3.2279088488,-2.6125621105,2.4036700717$ $\mathrm{H}, 0,2.9190953924,-1.3763549554,0.1269771386$ $\mathrm{H}, \mathrm{O}, 4.4772422574,-0.5609577915,-0.0030535251$ $\mathrm{H}, 0,-0.3780725984,1.7119607216,-1.1125742077$ $\mathrm{H}, 0,-0.4430297491,4.1160737945,-1.5543684247$ $\mathrm{H}, 0,0.7099300109,6.2887542534,-1.7581024127$ $\mathrm{H}, 0,3.1627614911,6.4438111041,-1.3486753038$ $\mathrm{H}, 0,4.4440169895,4.4348923723,-0.7409522215$ $\mathrm{H}, 0,-1.0553372724,-0.0969622902,0.2124540297$ $\mathrm{H}, \mathrm{O},-0.7530942374,-1.7766016897,-0.2986704503$ $\mathrm{H}, 0,-0.8979824187,-0.4938038004,-1.5292074179$ $\mathrm{H}, 0,4.6217462152,2.5966882536,3.2143396933$ $\mathrm{H}, 0,3.3671081404,2.5630371635,1.9744481847$ $\mathrm{H}, 0,4.4526116887,1.8599974168,-2.7553686877$ $\mathrm{H}, \mathrm{O}, 6.1666539133,1.8377074781,-4.5201679663$ $\mathrm{H}, 0,8.5818422768,1.9495619821,-3.9221651602$ $\mathrm{H}, 0,9.2673432002,2.0736106988,-1.5566790207$ $\mathrm{H}, 0,8.6937349943,2.1292385724,0.817441853$ $\mathrm{H}, 0,8.8642789973,1.3037104223,2.9754682533$ $\mathrm{H}, 0,8.2947891006,2.2204619638,4.3935405418$ $\mathrm{H}, 0,8.7920083475,3.0909787723,2.9196602739$ $\mathrm{Cl}, 0,-0.6107230592,-4.0387576462,2.2157895648$ $C, 0,0.2304117115,-1.8682119974,5.7045631661$ C, $0,0.8267604439,-3.1151100453,5.4822921132$ C, $0,-0.9494923944,-1.7956705405,6.4642564492$ C, $0,0.2701780835,-4.2639260602,6.0319352014$ $\mathrm{H}, \mathrm{O}, 1.7089994434,-3.175460622,4.85436808$ C, $0,-1.5023523897,-2.948358679,7.0036734728$ $\mathrm{H}, \mathrm{O},-1.4353286135,-0.8349347981,6.6009942397$ C, $0,-0.8938932873,-4.1851635097,6.7920752664$ $\mathrm{H}, \mathrm{O}, 0.7359421971,-5.2269934789,5.8477964388$ $\mathrm{H}, \mathrm{O},-2.4205093354,-2.8825774306,7.5790345814$ $\mathrm{H}, \mathrm{O},-1.3336469387,-5.0859944058,7.2088177297$ C, $0,0.896163709,1.7523298211,6.0443406135$ C, $0,1.8781185155,1.7237440587,7.0415879809$ C, $0,-0.003674259,2.8218796894,5.9968029173$ C, $0,1.9652190223,2.7555019396,7.9703976287$ $\mathrm{H}, 0,2.5671777789,0.885527284,7.0805697755$ C, $0,0.0800364869,3.8452159524,6.9336369134$ 
$\mathrm{H}, 0,-0.767151944,2.8427614813,5.2262562035$

$\mathrm{C}, 0,1.0666964246,3.8180426959,7.917382081$

$\mathrm{H}, 0,2.732531169,2.726748285,8.737782883$

$\mathrm{H}, 0,-0.6273598927,4.6675924745,6.895374587$

$\mathrm{H}, 0,1.133152234,4.6226341421,8.6431155058$

\section{TS-6- $\left(S_{\mathrm{a}}, S_{\mathrm{Rh}}\right)$}

Opt \& $\mathrm{WB} 97 \mathrm{XD} / \mathrm{SDD} / 6-31 \mathrm{G}(\mathrm{d}, \mathrm{p})$ in toluene (SMD model)

SCF Done: E (RwB97XD) $=-3069.24915152 \mathrm{a} \cdot \mathrm{u}$.

Zero-point correction $=0.846066$ Hartree/Particle

Sum of electronic and thermal Free Energies $=-3068.518182 \mathrm{a} \cdot \mathrm{u}$. d $358.15 \mathrm{~K}$

Imaginary Freq. $=-312.0528 \mathrm{~cm}^{-1}$

Sp a B3LYP-D3 (BJ)/def2-TZVPP in toluene (SMD model)

SCF Done: E (RB3LYP) $=-3071.32142659 \mathrm{a} \cdot \mathrm{u}$.

$\mathrm{Rh}, 0,1.0690229558,1.6039450164,-0.1991726783$

$0,0,2.0916115428,0.0869450215,-1.1776983794$

C, $0,2.1013520247,-1.1505149999,-0.8195589613$

C, $0,1.5578329736,-1.7074445923,0.3635117757$

C, $0,2.8070989434,-2.0596790084,-1.7024381953$

C, $0,1.3203213653,-3.1357134918,0.3887295606$

C, $0,2.8003242732,-3.4056788208,-1.545660709$

C, $0,1.9838202924,-3.9884916753,-0.5363595653$

$\mathrm{H}, 0,3.3504130956,-4.0441953076,-2.2298585105$

C, $0,0.8942553868,-5.9545975693,0.3813920527$

C, $0,0.1627486474,-5.114239484,1.2427391633$

C, $0,0.3632307745,-3.7534582588,1.2394406352$

$C, 0,1.7760014052,-5.3877291006,-0.5068220917$

$\mathrm{H}, 0,0.7380516665,-7.0287121965,0.3967245427$

$\mathrm{H}, 0,-0.5973877226,-5.5349424121,1.8939299621$

$\mathrm{H}, \mathrm{O},-0.2733673848,-3.1347477858,1.8601676392$

$\mathrm{H}, 0,2.3174091787,-6.0064583437,-1.2183116465$

C, $0,1.3589321072,-0.8754625242,1.5676606534$

C, $0,1.3074170975,-1.4796051477,2.8419046734$

C, $0,1.361157121,0.5461555329,1.5611696146$

C, $0,1.0942239877,-0.7609729297,4.009021864$

$\mathrm{H}, 0,1.4608264966,-2.5467820756,2.9299342119$

C, $0,1.1503638117,1.2617538111,2.7547750214$

C, $0,0.9635951013,0.6246018295,3.97170048$

$\mathrm{H}, 0,1.0503983154,-1.2914984294,4.9556535492$

$\mathrm{H}, 0,1.1733183269,2.3462372245,2.7164667909$

$\mathrm{H}, 0,0.7854927003,1.1975107053,4.876375903$

C, $0,2.9452247068,2.3666485736,0.1255719228$

C, $0,3.1435972111,1.4394684137,0.9780807035$

$\mathrm{Cl}, 0,3.7095697958,-1.3559091871,-3.0277441604$

C, $0,3.6753395835,3.4578174377,-0.5037741303$

C, $0,4.5939162834,4.2098663773,0.2410674663$

C, $0,3.488577597,3.754079303,-1.8596512584$

C, $0,5.3097508078,5.2374202423,-0.3630822011$

$\mathrm{H}, \mathrm{O}, 4.7380913399,3.9837776509,1.2931019573$

C, $0,4.2158644301,4.7748600972,-2.4606020085$

$\mathrm{H}, \mathrm{O}, 2.7810287209,3.164781781,-2.4345647528$

C, $0,5.1242714371,5.5225679338,-1.7140331836$

$\mathrm{H}, 0,6.0167011733,5.8161975743,0.2233688151$

$\mathrm{H}, \mathrm{O}, 4.0717086746,4.9884635409,-3.5152466671$

$\mathrm{H}, 0,5.6856562892,6.324416019,-2.1833437354$

C, $0,4.1204291895,0.5236449238,1.5450339884$ 
$C, 0,4.3287276027,0.3975455981,2.9229061552$

C, $0,4.8937252862,-0.2283142409,0.6505731953$

C, $0,5.3035294299,-0.4696643381,3.3974660343$

$\mathrm{H}, 0,3.7182197881,0.9710041854,3.6109787768$

C, $0,5.864244614,-1.0981469024,1.1343554448$

$\mathrm{H}, \mathrm{O}, 4.7174850217,-0.1223103036,-0.4144963524$

C, $0,6.0681118545,-1.2214313492,2.5058189133$

$\mathrm{H}, 0,5.4645610665,-0.5649890619,4.4666378332$

$\mathrm{H}, 0,6.45516387,-1.683472181,0.4372126107$

$\mathrm{H}, 0,6.8223591191,-1.9053839013,2.8827935928$

C, $0,-0.1792201739,2.5434735796,-1.8007912579$

C, $0,0.1624596055,3.5151214843,-0.8410480191$

C, $0,-0.3821177788,3.0940215053,0.4166857216$

C, $0,-1.1530765198,1.8997603338,0.1956660558$

C, $0,-1.0214430173,1.5449628876,-1.1655939267$

C, $0,-1.9789400741,1.1844200317,1.2299850323$

C, $0,-1.7953930758,0.5272199462,-1.9643949723$

$\mathrm{H}, 0,0.0954351566,2.5432855494,-2.8473425747$

$\mathrm{H}, 0,0.7866868656,4.3835631217,-1.0011611171$

$\mathrm{H}, \mathrm{O},-0.3269932671,3.6365321998,1.3501765733$

$0,0,-0.512988475,-1.7590368781,-2.7361839538$

$0,0,-3.5668521524,3.2844413779,1.9307597445$

C, $0,-2.1297398543,-0.8008249497,-1.3215547991$

C, $0,-1.4419958307,-1.9726292597,-1.7744044567$

C, $0,-1.7615218436,-3.2128171261,-1.2834104918$

C, $0,-2.758254683,-3.3527733608,-0.2865384683$

$C, 0,-3.0750304723,-4.6232848886,0.2619754627$

$C, 0,-4.0241695707,-4.7486749657,1.243728649$

C, $0,-4.7054842665,-3.6066514048,1.7219870528$

C, $0,-4.4275292047,-2.3679057845,1.201151169$

C, $0,-3.4527185929,-2.2070607637,0.1808782711$

C, $0,-3.1418964525,-0.9304229461,-0.3938301225$

$C, 0,-0.0265362953,-2.880470011,-3.4463745555$

$C, 0,-3.4256478013,1.2347623713,0.802765207$

C, $0,-3.9623275051,0.2575591248,-0.0027564951$

C, $0,-5.3096048413,0.3723874867,-0.4884813786$

C, $0,-5.894979925,-0.5883971302,-1.3537434649$

$C, 0,-7.1852175437,-0.4440569337,-1.8003417322$

$C, 0,-7.9553107899,0.6706664014,-1.4034325766$

C, $0,-7.4134927991,1.6209875348,-0.5753858722$

C, $0,-6.0812340093,1.5008601515,-0.1025120874$

C, $0,-5.5091224599,2.4962409593,0.7340159028$

C, $0,-4.2123346708,2.3740094266,1.1600313285$

C, $0,-4.2747934809,4.4413847872,2.3228556186$

$\mathrm{H}, \mathrm{O},-1.2394408085,-4.1011415617,-1.6193396621$

$\mathrm{H}, \mathrm{O},-2.538334025,-5.4941661099,-0.1031952088$

$\mathrm{H}, \mathrm{O},-4.2537317996,-5.7260611584,1.6576772413$

$\mathrm{H}, 0,-5.4508743664,-3.710777741,2.5043657049$

$\mathrm{H}, 0,-4.9534230541,-1.4947863438,1.5725245581$

$\mathrm{H}, 0,0.5101031691,-3.5760324401,-2.7954434075$

$\mathrm{H}, \mathrm{O}, 0.6659079456,-2.487926349,-4.1915435252$

$\mathrm{H}, \mathrm{O},-0.8467145713,-3.4091773456,-3.9490059031$

$\mathrm{H}, 0,-5.3093179146,-1.4467544025,-1.6642399958$

$\mathrm{H}, 0,-7.6155503319,-1.1893980622,-2.4618774619$

$\mathrm{H}, 0,-8.9759495324,0.7762447814,-1.758595419$

$\mathrm{H}, \mathrm{O},-7.9985088278,2.4852604937,-0.2721759886$

$\mathrm{H}, 0,-6.1162383871,3.3518472678,1.0071613837$

$\mathrm{H}, 0,-4.5954412657,5.0298149356,1.4544047691$ 
$\mathrm{H}, \mathrm{O},-3.5809780524,5.0347335567,2.9196262798$

$\mathrm{H}, 0,-5.1501710222,4.1899264213,2.9341509941$

$\mathrm{H}, 0,-1.8412290987,1.6663700861,2.1980658187$

$\mathrm{H}, 0,-1.64594004,0.1490819094,1.3258780082$

$\mathrm{H}, \mathrm{O},-2.7361050681,1.0218314837,-2.2423857908$

$\mathrm{H}, \mathrm{O},-1.2508028287,0.3426207669,-2.8906773851$

\section{INT-7- $\left(S_{a}\right)$}

Opt a wB97XD/SDD/6-31G $(\mathrm{d}, \mathrm{p})$ in toluene (SMD model)

SCF Done: $\mathrm{E}(\mathrm{RwB} 97 \mathrm{XD})=-3069.33326878 \mathrm{a} \cdot \mathrm{u}$.

Zero-point correction $=0.848760$ Hartree/Particle

Sum of electronic and thermal Free Energies $=-3068.598727 \mathrm{a} \cdot \mathrm{u}$. @ $358.15 \mathrm{~K}$

Sp a B3LYP-D3 (BJ)/def2-TZVPP in toluene (SMD model)

$\mathrm{SCF}$ Done: $\mathrm{E}(\mathrm{RB} 3 \mathrm{LYP})=-3071.39589533 \mathrm{a} \cdot \mathrm{u}$.

$\mathrm{Rh}, 0,0.6631888381,-1.2802065932,-0.2571851613$

$0,0,1.5564341475,-0.1442635608,-1.7317894387$

C, $0,2.1279187659,0.9673821041,-1.3685186832$

C, $0,1.9905691678,1.5173333836,-0.0948118188$

$C, 0,2.9381077176,1.6795032804,-2.318559528$

C, $0,2.6009117314,2.7620993726,0.2458668984$

C, $0,3.5128520862,2.8796193702,-2.0272263705$

C, $0,3.3569739732,3.4597383356,-0.7404409784$

$\mathrm{H}, 0,4.1142823182,3.3900376847,-2.7732133902$

C, $0,3.8436052353,5.244009809,0.8317907384$

C, $0,3.1062346147,4.5541500435,1.8188914677$

C, $0,2.5065037872,3.3512590139,1.5385375679$

C, $0,3.9662850106,4.6981681142,-0.4208691227$

$\mathrm{H}, 0,4.3148171906,6.1929281645,1.0675274574$

$\mathrm{H}, 0,3.0179720797,4.9768963585,2.815545929$

$\mathrm{H}, 0,1.955976485,2.8378835819,2.3196695628$

$\mathrm{H}, 0,4.5391272646,5.207944861,-1.1913925661$

C, $0,1.1986366994,0.7829847714,0.9432175752$

$C, 0,0.0112508279,1.3839406467,1.4537879231$

C, $0,1.7404419439,-0.320792975,1.6546186022$

C, $0,-0.5790784539,0.9508791024,2.6167142055$

$\mathrm{H}, 0,-0.3976771595,2.231028381,0.9163511769$

C, $0,1.1062552621,-0.7530041888,2.8528129617$

C, $0,-0.0160225379,-0.1227883006,3.3347152863$

$\mathrm{H}, \mathrm{O},-1.4711994407,1.4447316509,2.9892914294$

$\mathrm{H}, 0,1.5461714092,-1.5882559601,3.3899466051$

$\mathrm{H}, \mathrm{O},-0.4705510566,-0.4536644573,4.2634104944$

C, $0,2.5877749433,-1.7984577632,0.128302463$

C, $0,2.9720406127,-1.0314865721,1.1575007063$

$\mathrm{Cl}, 0,3.2039796366,0.946838176,-3.8813954046$

C, $0,3.2891287052,-2.8213047381,-0.6569140573$

C, $0,3.4314780511,-2.6892540661,-2.0452598889$

C, $0,3.7680843913,-3.9826073429,-0.0331773044$

$\mathrm{C}, 0,4.0539777355,-3.6895574266,-2.7847185865$

$\mathrm{H}, 0,3.0447140301,-1.795401906,-2.5245032632$

C, $0,4.3832088535,-4.9831076049,-0.7788684116$

$\mathrm{H}, 0,3.6604029078,-4.088600551,1.0426091574$

$\mathrm{C}, 0,4.5292375722,-4.8396592851,-2.1572229476$

$\mathrm{H}, 0,4.1653439283,-3.5697000504,-3.858371795$

$\mathrm{H}, 0,4.7510184775,-5.875872767,-0.2815842476$

$\mathrm{H}, \mathrm{O}, 5.0110623603,-5.6199312149,-2.7385929807$

C, 0,4.2945437747, $-0.7953751215,1.77341497$ 
C, $0,5.4797358145,-1.2526196811,1.1767287673$

$\mathrm{C}, 0,4.3964611107,-0.0536696498,2.9580770456$

C, $0,6.713933694,-0.9953991462,1.7594019599$

$\mathrm{H}, 0,5.4368018081,-1.8000338917,0.2424406934$

C, $0,5.6340791376,0.2053177876,3.5386170132$

$\mathrm{H}, 0,3.4991844042,0.3333639481,3.4310851132$

$\mathrm{C}, 0,6.7996453436,-0.2683083779,2.9452901633$

$\mathrm{H}, 0,7.616855067,-1.3571337247,1.2764556726$

$\mathrm{H}, 0,5.6850675693,0.7850418451,4.4554763589$

$\mathrm{H}, 0,7.766561294,-0.0647424837,3.3950751584$

$0,0,-1.110341921,1.9650149746,-1.8364732583$

$0,0,-0.9484161179,-5.2602999953,-3.3895575836$

$C, 0,-0.0902655319,-3.3058462052,-0.4884772864$

$\mathrm{C}, 0,-0.7015416299,-2.7163138579,0.6470410422$

$\mathrm{C}, 0,-1.5337026505,-1.6289292506,0.1726100625$

C, $0,-1.4088951936,-1.5116325688,-1.2148475911$

$\mathrm{C}, 0,-0.4267254435,-2.5142624776,-1.6334031437$

C, $0,-2.1882043137,-0.5580874208,-2.0834365525$

C, $0,-1.4228932801,0.0846641183,-3.2200652597$

$\mathrm{C}, 0,-0.890616332,1.4004183124,-3.0504221448$

$C, 0,-0.2315321168,2.0285285893,-4.0752130679$

$\mathrm{C}, 0,-0.0439516095,1.3726941574,-5.3174945398$

$C, 0,0.6537731529,2.0028286792,-6.3800096704$

C, $0,0.8574826961,1.3536564226,-7.5705344435$

$\mathrm{C}, 0,0.3744273937,0.0386035171,-7.7522173397$

$C, 0,-0.3063288136,-0.5937596874,-6.7420887786$

$\mathrm{C}, 0,-0.5394815551,0.0564377528,-5.5015620892$

$\mathrm{C}, 0,-1.2504703286,-0.5644989864,-4.4229880117$

$C, 0,-0.6629950502,3.2930786349,-1.6488032639$

C, $0,0.0448473782,-2.7462505519,-3.0424318966$

$C, 0,-1.13517356,-2.9941249999,-3.9526739852$

C, $0,-1.7723957094,-1.9551556866,-4.5930158029$

$\mathrm{C}, 0,-2.9302614095,-2.1980439616,-5.4033868479$

$C, 0,-3.6362827509,-1.1530481025,-6.0543863919$

$\mathrm{C}, 0,-4.7388075024,-1.4215740421,-6.8267718991$

C, $0,-5.1891005592,-2.7507367547,-6.986372438$

C, $0,-4.5317561977,-3.780737199,-6.3624233786$

$C, 0,-3.3919948301,-3.533150536,-5.554015309$

C, $0,-2.7211369053,-4.5915847843,-4.8858019528$

C, $0,-1.6337174535,-4.3260477868,-4.0933427942$

C, $0,-1.3491759503,-6.6088772051,-3.5119532313$

$\mathrm{H}, 0,0.59485319,-4.1428876116,-0.4956801593$

$\mathrm{H}, 0,-0.6246674478,-3.0549275525,1.6705279624$

$\mathrm{H}, \mathrm{O},-2.1166405943,-0.9711753343,0.8048771222$

$\mathrm{H}, \mathrm{O},-2.6026638095,0.2202719415,-1.4403888206$

$\mathrm{H}, \mathrm{O},-3.0342580461,-1.1137885517,-2.5043394588$

$\mathrm{H}, 0,0.1828386544,3.0220484501,-3.9532999685$

$\mathrm{H}, \mathrm{0}, 1.0361738848,3.0083808463,-6.2273973971$

$\mathrm{H}, \mathrm{0}, 1.3967327845,1.8463137165,-8.3739280048$

$\mathrm{H}, 0,0.5448919467,-0.4734492958,-8.6940972814$

$\mathrm{H}, 0,-0.6711851013,-1.6049682788,-6.8869917$

$\mathrm{H}, 0,0.4281943414,3.3640767318,-1.7144901257$

$\mathrm{H}, 0,-0.9821161843,3.5900789505,-0.6491059542$

$\mathrm{H}, \mathrm{0},-1.1229289341,3.9734196082,-2.3764040904$

$\mathrm{H}, \mathrm{O}, \mathrm{0} .7293369157,-3.5954289639,-3.052330449$

$\mathrm{H}, 0,0.5992602967,-1.8644942742,-3.3742759627$

$\mathrm{H}, \mathrm{O},-3.2925994356,-0.1309910453,-5.9357176074$

$\mathrm{H}, 0,-5.2667640131,-0.6102991154,-7.3179947694$ 
$\mathrm{H}, \mathrm{O},-6.0602944217,-2.9548683011,-7.6014255866$

$\mathrm{H}, 0,-4.8775661001,-4.8046593395,-6.476945539$

$\mathrm{H}, \mathrm{O},-3.0983031059,-5.6005415134,-5.0079904814$

$\mathrm{H}, 0,-2.3759251812,-6.7582096478,-3.1557335867$

$\mathrm{H}, \mathrm{O},-0.6671668947,-7.1864238105,-2.886894139$

$\mathrm{H}, 0,-1.26917766,-6.9584298732,-4.5485333234$

\section{INT-7- $\left(R_{a}\right)$}

Opt a wB97XD/SDD/6-31G $(\mathrm{d}, \mathrm{p})$ in toluene (SMD model)

SCF Done: $\mathrm{E}(\mathrm{RwB} 97 \mathrm{XD})=-3069.33216445 \mathrm{a} \cdot \mathrm{u}$.

Zero-point correction $=0.848823$ Hartree/Particle

Sum of electronic and thermal Free Energies $=-3068.599432 \mathrm{a} \cdot \mathrm{u}$. @ $358.15 \mathrm{~K}$

Sp a B3LYP-D3 (BJ)/def2-TZVPP in toluene (SMD model)

$\mathrm{SCF}$ Done: $\mathrm{E}(\mathrm{RB} 3 \mathrm{LYP})=-3071.39600015 \mathrm{a} \cdot \mathrm{u}$.

$\mathrm{Rh}, 0,1.8317822732,-0.4186266179,-0.2648325655$

$0,0,0.1628023335,-1.4614878594,-0.9140884081$

C, $0,-1.010269972,-0.9232860436,-1.0577300545$

C, $0,-1.2426295675,0.4495459615,-1.1906785616$

C, $0,-2.1580555796,-1.7900036108,-1.1389990866$

$C, 0,-2.5581350799,0.9660929002,-1.4016692636$

$C, 0,-3.4174973988,-1.3273840762,-1.3670976484$

$C, 0,-3.6564381779,0.065316593,-1.503521825$

$\mathrm{H}, \mathrm{O},-4.2499945375,-2.0210928719,-1.4343240431$

C, $0,-5.198486647,1.9121182786,-1.8202844105$

$\mathrm{C}, 0,-4.1192206159,2.8139897323,-1.6939478745$

C, $0,-2.8408203393,2.3584698702,-1.4874980963$

C, $0,-4.9646245535,0.5639631313,-1.7202432402$

$\mathrm{H}, \mathrm{O},-6.2041338925,2.2864592702,-1.9838130966$

$\mathrm{H}, \mathrm{O},-4.3033769992,3.882833147,-1.7518127492$

$\mathrm{H}, \mathrm{O},-2.0326627951,3.073286823,-1.3775225602$

$\mathrm{H}, 0,-5.7842510667,-0.1458422301,-1.7985782135$

C, $0,-0.0943540549,1.3901585505,-1.0881044426$

C, $0,0.2771696261,2.1788624725,-2.1957905205$

C, $0,0.5458288462,1.6157952191,0.153770208$

$\mathrm{C}, 0,1.2225481254,3.1800218284,-2.0780670732$

$\mathrm{H}, 0,-0.2107097355,2.0009299954,-3.1471779625$

C, $0,1.5314760552,2.6304656848,0.2440740395$

C, $0,1.8493688564,3.4159561674,-0.8453289586$

$\mathrm{H}, \mathrm{O}, 1.4839279969,3.7769340853,-2.9467613954$

$\mathrm{H}, 0,2.0131156929,2.7936910827,1.2039266656$

$\mathrm{H}, 0,2.5940883247,4.2002524848,-0.7548535705$

C, $0,0.782756445,-0.2929151971,1.4618493833$

C, $0,0.1083319538,0.860684884,1.383100572$

$0,0,0.7514944935,-1.6962395476,-3.9904450448$

$0,0,6.9398246724,1.439198689,-1.3692139218$

$C, 0,3.9194496339,-0.052453711,0.2548892651$

C, $0,3.5546229856,-1.3879480601,0.5640320651$

C, $0,3.1890641094,-2.0138839454,-0.6723271467$

C, $0,3.4676991073,-1.1141140698,-1.768054568$

C, $0,3.8853263018,0.1017373231,-1.1856184786$

C, $0,3.3678091401,-1.4764714614,-3.2297465076$

C, $0,2.7136385583,-0.4199396225,-4.0941634716$

C, $0,1.328154365,-0.5598493791,-4.4290085753$

C, $0,0.6821101796,0.4017566692,-5.1655884553$

C, $0,1.3739235471,1.5654742123,-5.5899609983$

C, 0, $0.707952697,2.6033790105,-6.2924411724$ 
C, $0,1.3812493419,3.7342296581,-6.6815515878$ $\mathrm{C}, 0,2.7555836981,3.8787248339,-6.3887663713$ C, $0,3.4262395462,2.8877557811,-5.7148465217$ $C, 0,2.7557623214,1.7093640417,-5.2964598385$ $C, 0,3.4136943523,0.6710553425,-4.5585845263$ $C, 0,-0.6254357558,-1.8887089349,-4.2433043782$ C, $0,4.257361827,1.3691780121,-1.9002633997$ C, $0,5.2748421687,1.1095064968,-2.9843580567$ $C, 0,4.8731723437,0.784368561,-4.2585038725$ C, $0,5.8386594997,0.5129159368,-5.2828654475$ C, $0,5.4678823457,0.1539812645,-6.6048101312$ C, $0,6.421728884,-0.0958893874,-7.5598452964$ C, $0,7.7935341214,-0.0018340766,-7.2371997475$ C, $0,8.181434562,0.3333879859,-5.9646383428$ $C, 0,7.2185602135,0.5942564924,-4.9555241053$ $C, 0,7.6135863569,0.9183327633,-3.6299568737$ $C, 0,6.666786332,1.1542624509,-2.6662479062$ C, $0,8.2944487495,1.4668140831,-0.9683188046$ $\mathrm{H}, 0,4.2529977617,0.7055803417,0.9499769511$ $\mathrm{H}, \mathrm{O}, 3.4965234032,-1.8358773044,1.5462343088$ $\mathrm{H}, 0,2.8171172279,-3.0249342823,-0.780576669$ $\mathrm{H}, 0,2.8047110264,-2.4068021972,-3.309793949$ $\mathrm{H}, 0,4.3790359563,-1.6745298948,-3.6030309356$ $\mathrm{H}, 0,-0.3665150743,0.302109799,-5.4209287764$ $\mathrm{H}, \mathrm{O},-0.350151899,2.4893537592,-6.5127098266$ $\mathrm{H}, 0,0.8580297037,4.5223153499,-7.2145078102$ $\mathrm{H}, 0,3.2821820646,4.7760313501,-6.6983433003$ $\mathrm{H}, 0,4.481416474,3.0031875974,-5.4895091023$ $\mathrm{H}, \mathrm{O},-1.2271498056,-1.0767678964,-3.820981246$ $\mathrm{H}, \mathrm{O},-0.8930194099,-2.8244068056,-3.753414071$ $\mathrm{H}, 0,-0.8225161002,-1.9720228768,-5.3198872806$ $\mathrm{H}, 0,4.648774182,2.082825195,-1.1743471706$ $\mathrm{H}, \mathrm{O}, 3.3630849333,1.8136147799,-2.3438415582$ $\mathrm{H}, 0,4.4154141167,0.0791772977,-6.8571516204$ $\mathrm{H}, \mathrm{O}, 6.1220494295,-0.3682875748,-8.5668498841$ $\mathrm{H}, 0,8.5413033805,-0.199649982,-7.9991591431$ $\mathrm{H}, 0,9.2357471438,0.4007088049,-5.7098531793$ $\mathrm{H}, 0,8.672935598,0.9589158299,-3.4039522672$ $\mathrm{H}, 0,8.7800748768,0.4970711234,-1.1315647107$ $\mathrm{H}, 0,8.2905041896,1.6898375567,0.0991995102$ $\mathrm{H}, 0,8.855261972,2.2484003254,-1.4956441526$ $\mathrm{Cl}, 0,-1.8933355659,-3.5127531328,-0.9639652873$ $C, 0,0.7722335708,-1.340356926,2.4902117661$ C, $0,0.4963280001,-2.6700344784,2.1405272867$ C, $0,1.0696765217,-1.0389073654,3.8261707966$ C, $0,0.4988399746,-3.6671473403,3.1099105391$ $\mathrm{H}, 0,0.2678791983,-2.8970848621,1.1031097893$ C, $0,1.0797821219,-2.0417000369,4.7907292632$ $\mathrm{H}, 0,1.283236704,-0.0106276477,4.1028235667$ C, $0,0.7925362458,-3.3583527167,4.437174492$ $\mathrm{H}, 0,0.2695764112,-4.6904642693,2.8272614462$ $\mathrm{H}, 0,1.3098310404,-1.7930061061,5.822640875$ $\mathrm{H}, 0,0.7980124605,-4.1392708201,5.1915386429$ C, $0,-0.9885978754,1.3971974899,2.2205923064$ C, $0,-1.8267229424,0.5452105294,2.9544610763$ C, $0,-1.2506109907,2.7727192377,2.2494895532$ C, $0,-2.8706970911,1.0587416818,3.7134927168$ $\mathrm{H}, \mathrm{O},-1.6693207457,-0.5268933425,2.9143641002$ 
$C, 0,-2.2982478489,3.2852040625,3.0082619507$

$\mathrm{H}, 0,-0.630992389,3.4512169746,1.6702914414$

C, $0,-3.1103109768,2.4311971423,3.7479309457$

$\mathrm{H}, 0,-3.5107555671,0.3804244824,4.2697923287$

$\mathrm{H}, \mathrm{O},-2.4821303195,4.3555104749,3.0159733151$

$\mathrm{H}, \mathrm{O},-3.9308541639,2.8293142557,4.3371025525$

\section{TS-7- $\left(S_{\mathrm{a}}\right)$}

Opt a wB97XD/SDD/6-31G $(\mathrm{d}, \mathrm{p})$ in toluene (SMD model)

SCF Done: $\mathrm{E}(\mathrm{RwB} 97 \mathrm{XD})=-3069.29957569 \mathrm{a} \cdot \mathrm{u}$.

Zero-point correction $=0.846925$ Hartree/Particle

Sum of electronic and thermal Free Energies $=-3068.567346 \mathrm{a} \cdot \mathrm{u}$. d $358.15 \mathrm{~K}$

Imaginary Freq. $=-116.6235 \mathrm{~cm}^{-1}$

Sp a B3LYP-D3 (BJ)/def2-TZVPP in toluene (SMD model)

SCF Done: E (RB3LYP) $=-3071.37001090 \mathrm{a} \cdot \mathrm{u}$.

$\mathrm{Rh}, 0,-0.7551023866,-1.0355735573,-0.6874414606$

$0,0,-0.3909630679,1.0846423222,-0.4035742171$

C, $0,-1.2699733858,1.6581175524,0.3375405458$

$C, 0,-2.6435759131,1.707147231,-0.0088390197$

$C, 0,-0.910415683,2.2181515922,1.6242667229$

C, $0,-3.6361009495,2.1620368529,0.9184772006$

C, $0,-1.8457403172,2.6070891478,2.5292190515$

C, $0,-3.2398486172,2.5577680707,2.2202705359$

$\mathrm{H}, \mathrm{O},-1.537785575,2.9539359983,3.511120237$

C, $0,-5.5558818147,2.8669827773,2.8590809954$

C, $0,-5.9545958009,2.4837275485,1.5649568729$

C, $0,-5.0174208963,2.1389611317,0.6150661351$

C, $0,-4.2163573834,2.9054163344,3.1768356627$

$\mathrm{H}, \mathrm{O},-6.30213284,3.1316202756,3.6014906285$

$\mathrm{H}, 0,-7.0107390706,2.453918363,1.3137708227$

$\mathrm{H}, 0,-5.3336655554,1.8314480023,-0.376807494$

$\mathrm{H}, \mathrm{O},-3.8943120795,3.2008797906,4.1718229209$

C, $0,-2.9695152161,1.5004124736,-1.4400014516$

C, $0,-3.1990477747,2.5858554688,-2.2892885606$

C, $0,-2.9919976305,0.1975345737,-1.9612975863$

$C, 0,-3.4209152454,2.3926326044,-3.64802881$

$\mathrm{H}, \mathrm{O},-3.1982559653,3.5886222874,-1.8721519521$

$C, 0,-3.1592020865,0.0133109263,-3.341372827$

C, $0,-3.379750964,1.1012209008,-4.174948134$

$\mathrm{H}, \mathrm{O},-3.6076668445,3.2437672265,-4.2952295155$

$\mathrm{H}, \mathrm{O},-3.1340934127,-0.9912156217,-3.752155811$

$\mathrm{H}, \mathrm{O},-3.5233119003,0.9415247966,-5.239361571$

$C, 0,-2.3968237315,-0.7136449247,0.2936275866$

C, $0,-2.9315404048,-0.9289985384,-0.9956312541$

$C, 0,-3.8935907902,-2.0720327229,-1.1614806565$

C, $0,-5.0696896745,-1.937658935,-1.9115977395$

$C, 0,-3.6983505741,-3.2756920659,-0.4681255761$

C, $0,-5.9932312843,-2.9763407882,-1.9907892595$

$\mathrm{H}, 0,-5.2762817094,-1.0095494027,-2.4325241937$

C, $0,-4.6209395836,-4.3115282542,-0.5432777533$

$\mathrm{H}, \mathrm{O},-2.8185709693,-3.3942249966,0.1539506449$

C, $0,-5.7728339185,-4.1708617209,-1.3131248688$

$\mathrm{H}, 0,-6.8951644348,-2.8422585293,-2.5805628255$

$\mathrm{H}, \mathrm{O},-4.4389705259,-5.2309777884,0.0050870679$

$\mathrm{H}, \mathrm{O},-6.4933500998,-4.9802502838,-1.3774065231$

$C, 0,-2.8619877567,-0.9093170762,1.6450975659$ 
C, $0,-4.2374295966,-0.9033099649,1.9432758104$ C, $0,-1.9364709872,-0.9845892601,2.6983656848$ $\mathrm{C}, 0,-4.6674472975,-0.9932783801,3.2579576559$ $\mathrm{H}, \mathrm{O},-4.9569612008,-0.8260137962,1.1354693315$ $\mathrm{C}, 0,-2.3723508628,-1.0781606648,4.0136275429$ $\mathrm{H}, \mathrm{O},-0.8768179977,-0.9481150672,2.4644495379$ $\mathrm{C}, 0,-3.7370939422,-1.0830036156,4.2943320254$ $\mathrm{H}, 0,-5.7297244837,-0.9837459182,3.4793147175$ $\mathrm{H}, \mathrm{0},-1.6484273926,-1.1331987267,4.8204992121$ $\mathrm{H}, \mathrm{O},-4.0780024083,-1.1476543913,5.3231793315$ $\mathrm{Cl}, 0,0.7807289682,2.3088986083,2.0411011824$ $0,0,1.0879904653,1.2259327601,-3.1242435825$ $0,0,3.4298988838,-3.140979008,2.243679573$ $C, 0,0.2601558168,-2.7259950363,0.3727626167$ $C, 0,-0.3104425216,-3.1336201923,-0.8833503114$ C, $0,0.4498645232,-2.5195126064,-1.9349456946$ $\mathrm{C}, 0,1.3798906321,-1.6516377812,-1.3385510559$ C, $0,1.2652716669,-1.7715913117,0.1089884784$ C, $0,2.4313583519,-0.8794714751,-2.0911206402$ $C, 0,2.6966560444,0.5174169805,-1.5833813758$ $\mathrm{C}, 0,1.9667815825,1.5958086094,-2.165813644$ $\mathrm{C}, 0,2.1794423205,2.8880417457,-1.7618476936$ C, $0,3.1049995048,3.1673955191,-0.7247097777$ C, $0,3.3194864502,4.4945653068,-0.2693958188$ $\mathrm{C}, 0,4.1846626563,4.7553124072,0.7624458232$ C, 0,4.8748919389,3.6968805507,1.3937899073 C, $0,4.692820984,2.4038686521,0.9699715018$ $\mathrm{C}, 0,3.8143651428,2.1042218311,-0.1038104931$ $\mathrm{C}, 0,3.5955823538,0.7671876182,-0.5714304653$ C, $0,0.1093290361,2.1666378034,-3.5160901193$ C, $0,2.1008054853,-1.0364753755,1.1222380819$ $\mathrm{C}, 0,3.5817631171,-1.2341222447,0.8888769427$ $\mathrm{C}, 0,4.299046444,-0.3796527099,0.0801215563$ $\mathrm{C}, 0,5.6938928663,-0.6045413496,-0.1662686196$ $\mathrm{C}, 0,6.4609359863,0.2331575831,-1.0174588256$ $\mathrm{C}, 0,7.7957162056,-0.0078916827,-1.2281392676$ C, $0,8.4285757466,-1.10294801,-0.5994352082$ $\mathrm{C}, 0,7.7101632798,-1.9364566322,0.2200296042$ C, $0,6.328269038,-1.713803596,0.4540870778$ C, $0,5.5734373286,-2.583688032,1.2848200335$ $\mathrm{C}, 0,4.2356918835,-2.3564427053,1.4852484529$ C, $0,4.0055099996,-4.2630243043,2.877765405$ $\mathrm{H}, \mathrm{O},-0.0550580614,-3.0578990277,1.3531241205$ $\mathrm{H}, \mathrm{O},-1.073963561,-3.8850898402,-1.0259258299$ $\mathrm{H}, \mathrm{O}, \mathrm{0} .3022988533,-2.65698043,-2.9973611127$ $\mathrm{H}, 0,2.1394201596,-0.837067059,-3.141348532$ $\mathrm{H}, 0,3.3575203079,-1.4620704773,-2.0348267815$ $\mathrm{H}, 0,1.6280835677,3.7116138166,-2.2008833249$ $\mathrm{H}, 0,2.7749519669,5.3037890801,-0.7486955351$ $\mathrm{H}, 0,4.3343957794,5.7757359204,1.1024495084$ $\mathrm{H}, 0,5.5495137242,3.9075847488,2.2178541889$ $\mathrm{H}, 0,5.2232588937,1.5945051282,1.4608027824$ $\mathrm{H}, \mathrm{O},-0.4194405209,2.555738056,-2.6415325141$ $\mathrm{H}, \mathrm{O},-0.5934254529,1.6258657154,-4.1494846109$ $\mathrm{H}, 0,0.5509725062,2.9918534467,-4.0901965533$ $\mathrm{H}, 0,1.8295149841,-1.3862958634,2.1204513603$ $\mathrm{H}, 0,1.8626277889,0.0274299387,1.0666566925$ $\mathrm{H}, 0,5.9778318663,1.0747730865,-1.50236137$ 
$\mathrm{H}, 0,8.3677624266,0.6442169665,-1.8807317164$

$\mathrm{H}, 0,9.4852792195,-1.2857412235,-0.7697905838$

$\mathrm{H}, 0,8.1910575943,-2.7842372559,0.7008054795$

$\mathrm{H}, 0,6.0781177413,-3.4274520486,1.7416185602$

$\mathrm{H}, 0,4.4151345858,-4.9727180102,2.1483676532$

$\mathrm{H}, 0,3.1992754135,-4.7467400186,3.4307406175$

$\mathrm{H}, 0,4.7952975587,-3.9678713103,3.579712351$

\section{TS-7- $\left(R_{a}\right)$}

Opt a wB97XD/SDD/6-31G (d,p) in toluene (SMD model)

SCF Done: E (RwB97XD) $=-3069.30397098$ a.u.

Zero-point correction $=0.847537$ Hartree/Particle

Sum of electronic and thermal Free Energies $=-3068.568658 \mathrm{a} \cdot \mathrm{u}$. @ $358.15 \mathrm{~K}$

Imaginary Freq. $=-223.4771 \mathrm{~cm}^{-1}$

Sp a B3LYP-D3 (BJ)/def2-TZVPP in toluene (SMD model)

$\mathrm{SCF}$ Done: $\mathrm{E}(\mathrm{RB} 3 \mathrm{LYP})=-3071.36998236 \mathrm{a} \cdot \mathrm{u}$.

$\mathrm{Rh}, 0,0.6862414264,0.731107448,-0.7874532303$

$0,0,1.8050051941,-0.5356041003,-2.178106061$

C, $0,3.0315599467,-0.5096426694,-1.817971605$

$\mathrm{C}, 0,3.4783761481,-1.1541161744,-0.6297009419$

C, $0,4.0168399964,0.2634250275,-2.5466199678$

C, $0,4.8370028711,-1.0398591915,-0.1786591441$

C, $0,5.2800034934,0.4424042029,-2.0837704481$

C, $0,5.7265993149,-0.1876696256,-0.8780381586$

$\mathrm{H}, \mathrm{O}, 5.9701002605,1.0833458582,-2.6243594718$

$\mathrm{C}, 0,7.4484495525,-0.5867666493,0.7770836464$

C, $0,6.5743794906,-1.4450907587,1.46613044$

C, $0,5.2943055815,-1.6654734364,1.0015356321$

C, $0,7.0272103776,0.0300599739,-0.3812559457$

$\mathrm{H}, 0,8.4506477422,-0.4130021179,1.1554673461$

$\mathrm{H}, 0,6.9063469014,-1.9326625579,2.3778108215$

$\mathrm{H}, 0,4.6202825868,-2.3161626136,1.549619379$

$\mathrm{H}, 0,7.6930372904,0.6953726848,-0.9243219395$

C, $0,2.5584232699,-2.178169063,-0.0807013761$

C, $0,2.7478369552,-3.5413222751,-0.3101793798$

C, $0,1.439328716,-1.7409048894,0.6433402063$

C, $0,1.8271046785,-4.4709017064,0.1658245119$

$\mathrm{H}, \mathrm{O}, 3.6238434543,-3.8692787956,-0.8622504125$

C, $0,0.4900638702,-2.6746225075,1.0700449994$

C, $0,0.6914148131,-4.0305674277,0.8438209576$

$\mathrm{H}, 0,1.9887157575,-5.5309252651,-0.0036103808$

$\mathrm{H}, \mathrm{O},-0.3953366628,-2.3404270187,1.6008856548$

$\mathrm{H}, \mathrm{O},-0.0463868988,-4.7438025426,1.1989694544$

C, $0,2.2752854228,0.5962404671,0.347933645$

C, $0,1.3937421375,-0.3029666156,1.0100762718$

C, $0,1.0180347867,0.0184332281,2.4332092902$

C, $0,1.2340589516,-0.9098093477,3.4625010282$

C, $0,0.5470808728,1.2852971229,2.7926549688$

C, $0,0.9663933748,-0.5868073373,4.7889630689$

$\mathrm{H}, 0,1.6251517332,-1.8936329607,3.2282525771$

$C, 0,0.2812065525,1.6126569778,4.1172153162$

$\mathrm{H}, 0,0.3913505432,2.0239789457,2.0196856851$

C, $0,0.4833272573,0.6745332001,5.124767148$

$\mathrm{H}, \mathrm{O}, 1.1420225123,-1.3273072784,5.5636292383$

$\mathrm{H}, \mathrm{O},-0.088700334,2.6045256493,4.3596577543$

$\mathrm{H}, 0,0.2708927052,0.9243771802,6.1596073638$ 
$C, 0,3.3011583692,1.5105719517,0.8001949621$ $C, 0,4.0233815779,1.2567247661,1.9807106082$ C, $0,3.6761891709,2.5970217978,-0.0045424663$ $C, 0,5.0790739621,2.0771491536,2.3457961573$ $\mathrm{H}, 0,3.7459619497,0.4119917475,2.6016565788$ C, $0,4.7321008754,3.4202799344,0.3668538059$ $\mathrm{H}, 0,3.1366279254,2.7664062418,-0.930274621$ C, 0,5.436181873,3.1589758138,1.5397201875 $\mathrm{H}, 0,5.6331602231,1.8698515621,3.2556094043$ $\mathrm{H}, 0,5.0143778605,4.2573558629,-0.2639778806$ $\mathrm{H}, 0,6.2680410837,3.7952546599,1.8263664909$ $\mathrm{Cl}, 0,3.4951737056,1.0676423166,-4.0059566824$ $0,0,-1.0699024901,-2.0280136072,-2.6998263221$ $0,0,-3.6176642156,3.6944448323,1.1246196898$ C, $0,-0.2052502163,2.6702863493,-0.359990994$ $C, 0,0.3465138261,2.7778295303,-1.6737745013$ C, $0,-0.3196251955,1.8126555124,-2.4618326298$ $C, 0,-1.3848525116,1.2084628531,-1.7002812158$ C, $0,-1.3060861918,1.7265730517,-0.3877656839$ C, $0,-2.4217398822,0.2790134498,-2.2726572549$ C, $0,-2.6830139423,-0.9304955274,-1.4093592449$ $C, 0,-1.9354556721,-2.1209254456,-1.6679890069$ $C, 0,-2.121581905,-3.244652326,-0.9052265565$ C, $0,-3.0220497833,-3.2222250726,0.1909362694$ C, $0,-3.1878143853,-4.3568933198,1.0271896066$ C, $0,-4.0338868555,-4.3199690498,2.1072038247$ $C, 0,-4.7552701792,-3.1423082467,2.4023538063$ C, $0,-4.6183841041,-2.0305446824,1.6079411544$ C, $0,-3.7539991271,-2.0394325721,0.4830361922$ C, $0,-3.5745616637,-0.8945758144,-0.3617772461$ C, $0,-0.1003111622,-3.0463905272,-2.8516928767$ $\mathrm{C}, 0,-2.1991724867,1.3843861627,0.7833617102$ $C, 0,-3.6679158359,1.4552666654,0.4312818073$ C, $0,-4.3291754481,0.3688603104,-0.0973050704$ C, $0,-5.7171371425,0.4564526889,-0.448638301$ C, $0,-6.4228711558,-0.6267181198,-1.0340662467$ C, $0,-7.75121236,-0.5089350787,-1.3595003509$ $C, 0,-8.438024074,0.7011087057,-1.1170369698$ C, $0,-7.7785207165,1.7691609587,-0.5630402159$ C, $0,-6.4042148097,1.678336119,-0.2209002957$ C, $0,-5.7067968429,2.7882483362,0.3252885225$ $C, 0,-4.3728537151,2.6836521313,0.6274528101$ $\mathrm{C}, 0,-4.2366729582,4.9466334346,1.32899089$ $\mathrm{H}, 0,0.0545014077,3.2981308683,0.4818089375$ $\mathrm{H}, 0,1.1471374688,3.4296876986,-1.9917728581$ $\mathrm{H}, \mathrm{O},-0.0923939396,1.5679422191,-3.4915564124$ $\mathrm{H}, \mathrm{O},-2.0877167228,-0.0423583639,-3.2598754488$ $\mathrm{H}, 0,-3.3529395021,0.8389862155,-2.4107501114$ $\mathrm{H}, \mathrm{O},-1.5569909622,-4.1496445857,-1.0954060899$ $\mathrm{H}, \mathrm{O},-2.6300429844,-5.2604265046,0.7944392467$ $\mathrm{H}, \mathrm{O},-4.1489120736,-5.1957191767,2.7388803849$ $\mathrm{H}, \mathrm{O},-5.4197554241,-3.1170826538,3.2603811904$ $\mathrm{H}, \mathrm{O},-5.1733947404,-1.1273199347,1.8393286815$ $\mathrm{H}, 0,0.4710218262,-3.1852158715,-1.9294911707$ $\mathrm{H}, 0,0.5731251197,-2.7060438842,-3.6369996851$ $\mathrm{H}, 0,-0.5633771169,-3.99518774,-3.153087973$ $\mathrm{H}, 0,-1.9853499138,2.0710903951,1.602581288$ $\mathrm{H}, \mathrm{O},-1.9659723001,0.3786670423,1.1399932848$ 
$\mathrm{H}, \mathrm{O},-5.8977598878,-1.5566108583,-1.2247468734$

$\mathrm{H}, 0,-8.2759786536,-1.3473960453,-1.8065665109$

$\mathrm{H}, \mathrm{O},-9.4892238904,0.7854363486,-1.3758083491$

$\mathrm{H}, 0,-8.3006943233,2.7050709708,-0.3822966471$

$\mathrm{H}, \mathrm{O},-6.2510522654,3.7128055633,0.480977751$

$\mathrm{H}, \mathrm{O},-4.6254370921,5.3607583468,0.3906766727$

$\mathrm{H}, 0,-3.4620008581,5.6095323963,1.716596918$

$\mathrm{H}, \mathrm{O},-5.0499851899,4.8805250032,2.0623299873$

\section{INT-8- ( $S)$}

Opt a wB97XD/SDD/6-31G $(\mathrm{d}, \mathrm{p})$ in toluene (SMD model)

SCF Done: $\mathrm{E}(\mathrm{RwB} 97 \mathrm{XD})=-3069.33379991 \mathrm{a} \cdot \mathrm{u}$.

Zero-point correction $=0.849302$ Hartree/Particle

Sum of electronic and thermal Free Energies $=-3068.597567 \mathrm{a} . \mathrm{u}$. d $358.15 \mathrm{~K}$

Sp \& B3LYP-D3 (BJ)/def2-TZVPP in toluene (SMD model)

$\mathrm{SCF}$ Done: $\mathrm{E}(\mathrm{RB} 3 \mathrm{LYP})=-3071.38657607 \mathrm{a} \cdot \mathrm{u}$.

$\mathrm{Rh}, 0,-0.843562225,-0.9221112659,-0.6320693706$

$0,0,-0.3535139075,1.1485631256,-0.1249222464$

$C, 0,-1.260173665,1.7474057705,0.4656065716$

$C, 0,-2.6726550965,1.2707332575,0.2282931306$

$C, 0,-1.0079167415,2.8385031075,1.3928256174$

$C, 0,-3.7260708313,1.898224634,1.1090700965$

C, $0,-2.0032845722,3.3283871857,2.1593920291$

$C, 0,-3.3797836071,2.8781453841,2.0506148945$

$\mathrm{H}, 0,-1.7799834665,4.1011352944,2.8899185711$

C, $0,-5.695272686,3.0714885098,2.7221931947$

C, $0,-6.0426080368,2.1191336216,1.7629424312$

$C, 0,-5.0656601763,1.5450314016,0.9600638629$

C, $0,-4.3710391229,3.4550280033,2.8582439168$

$\mathrm{H}, 0,-6.4585254012,3.5176721735,3.3511290596$

$\mathrm{H}, 0,-7.0802335212,1.8254827246,1.6400767972$

$\mathrm{H}, 0,-5.3412400986,0.8141133029,0.2059870584$

$\mathrm{H}, \mathrm{O},-4.0901268547,4.20982341,3.587231763$

C, $0,-2.9603163571,1.5634036069,-1.2392774644$

C, $0,-3.0710794812,2.8107732185,-1.8367423528$

$C, 0,-3.0576406221,0.3757813938,-1.9699077626$

C, $0,-3.2846365615,2.8795689715,-3.2119258756$

$\mathrm{H}, \mathrm{O},-3.0058005241,3.7163537661,-1.2391940611$

$C, 0,-3.2177374392,0.4557228418,-3.3581228453$

C, $0,-3.341224706,1.7035689434,-3.9630751502$

$\mathrm{H}, \mathrm{O},-3.3951172771,3.8433601998,-3.6985530708$

$\mathrm{H}, \mathrm{O},-3.2403871484,-0.4451733298,-3.9622168391$

$\mathrm{H}, \mathrm{O},-3.482400203,1.7604305998,-5.0385285237$

$C, 0,-2.6215369038,-0.3183631119,0.2668721911$

C, $0,-2.9451541713,-0.7999255387,-1.0726948167$

$C, 0,-3.7725994797,-2.0258195307,-1.3160362144$

C, $0,-4.8311253286,-2.0095424176,-2.2357411289$

C, $0,-3.6140433802,-3.1946199588,-0.5531130069$

$C, 0,-5.6563658663,-3.1173627254,-2.4186064817$

$\mathrm{H}, \mathrm{O},-5.0361405619,-1.1134615635,-2.8091515998$

C, $0,-4.4366507977,-4.299225388,-0.7296427646$

$\mathrm{H}, 0,-2.8513204074,-3.2267402543,0.213868386$

C, $0,-5.4600148356,-4.2742066113,-1.6746879382$

$\mathrm{H}, \mathrm{O},-6.4620173375,-3.0649993233,-3.1452894771$

$\mathrm{H}, \mathrm{O},-4.279188159,-5.1827739225,-0.1178679974$

$\mathrm{H}, \mathrm{O},-6.0994065987,-5.1396237727,-1.8190013541$ 
C, $0,-2.9987656169,-0.9100993368,1.592102264$

C, $0,-4.2798615776,-1.3993144231,1.8684348407$

$\mathrm{C}, 0,-2.0634235717,-0.8894637845,2.6337904355$ C, $0,-4.60670337,-1.8724211017,3.1360145925$ $\mathrm{H}, \mathrm{0},-5.0273636835,-1.4192559072,1.0831471085$ $\mathrm{C}, 0,-2.3903937522,-1.3476143867,3.9056520394$ $\mathrm{H}, \mathrm{O},-1.0605636381,-0.5229354318,2.4307526497$ C, $0,-3.6655124723,-1.8466861955,4.1608495172$ $\mathrm{H}, \mathrm{O},-5.6056566671,-2.2552666437,3.3227960131$ $\mathrm{H}, \mathrm{O},-1.6453748669,-1.3202328576,4.6955212247$ $\mathrm{H}, \mathrm{0},-3.9235080606,-2.2113584791,5.150537966$ $\mathrm{Cl}, 0,0.6233417115,3.408264969,1.554475057$ $0,0,1.0990275758,1.3932956989,-2.9817488748$ $0,0,3.2444789301,-3.2346754609,2.2716214945$ $\mathrm{C}, 0,0.1377250759,-2.6773863106,0.4188797974$ $\mathrm{C}, 0,-0.4549171871,-3.0339809242,-0.8479576758$ $\mathrm{C}, 0,0.3196042187,-2.4321316374,-1.8923367702$ C, $0,1.2541782838,-1.5812271037,-1.2795183145$ C, $0,1.1580724069,-1.7477381014,0.1702067339$ $\mathrm{C}, 0,2.3239699758,-0.8287862199,-2.0288098678$ $\mathrm{C}, 0,2.6827286144,0.5348403007,-1.4898054164$ $\mathrm{C}, 0,2.0308103316,1.6781040572,-2.0440101762$ $\mathrm{C}, 0,2.3580936012,2.9448096562,-1.6356808661$ C, $0,3.3242648096,3.1368126058,-0.6148730591$ C, $0,3.6585438021,4.4372999267,-0.1553882034$ $\mathrm{C}, 0,4.5574935607,4.6150157212,0.8660906025$ $\mathrm{C}, 0,5.1638247943,3.4962240342,1.4787513186$ C, $0,4.8644309014,2.2269982536,1.0495402281$ C, $0,3.9457173731,2.0117436877,-0.0103678373$ $\mathrm{C}, 0,3.6129019555,0.7015589123,-0.4891654594$ C, $0,0.2213277404,2.4254091683,-3.3763617941$ C, $0,2.0223895871,-1.0521409183,1.1907487062$ $\mathrm{C}, 0,3.4902100245,-1.321094472,0.940515716$ C, $0,4.2459821711,-0.503143944,0.1294734458$ $\mathrm{C}, 0,5.6158046516,-0.8190858962,-0.1575547342$ $C, 0,6.4156735915,-0.0255207603,-1.0208279587$ $\mathrm{C}, 0,7.7223705583,-0.3591362987,-1.2773159721$ C, $0,8.292983166,-1.5066982665,-0.684107606$ $\mathrm{C}, 0,7.5410569398,-2.2987902588,0.1463970908$ C, $0,6.1868338569,-1.9807755517,0.4268242704$ $\mathrm{C}, 0,5.3956455932,-2.808714354,1.2664155234$ C, $0,4.0821153464,-2.4943089981,1.5047511678$ $\mathrm{C}, 0,3.7490560268,-4.4182471482,2.8515431674$ $\mathrm{H}, \mathrm{O},-0.1884710037,-3.0152641215,1.3938423874$ $\mathrm{H}, \mathrm{O},-1.2224868462,-3.777113596,-1.0036973484$ $\mathrm{H}, 0,0.1751261433,-2.5563893176,-2.9566548174$ $\mathrm{H}, 0,2.0070474882,-0.7330507468,-3.0685023615$ $\mathrm{H}, 0,3.2235076879,-1.4548679671,-2.019901054$ $\mathrm{H}, 0,1.8720493176,3.8162449746,-2.059669942$ $\mathrm{H}, 0,3.1797726751,5.2935774292,-0.6231247262$ $\mathrm{H}, 0,4.8013752555,5.616187714,1.2088478$ $\mathrm{H}, 0,5.8684130385,3.6412708665,2.2917331089$ $\mathrm{H}, 0,5.3321030745,1.3711497637,1.5246897656$ $\mathrm{H}, \mathrm{O},-0.2650664319,2.8807910289,-2.5070977439$ $\mathrm{H}, \mathrm{O},-0.5360535488,1.9553401748,-4.0026904632$ $\mathrm{H}, 0,0.740950375,3.200019687,-3.9555182144$ $\mathrm{H}, 0,1.7418847353,-1.404977718,2.1851747696$ $\mathrm{H}, 0,1.8387551577,0.024716889,1.1540478952$ 
$\mathrm{H}, 0,5.9802333968,0.8549899709,-1.4813685936$ $\mathrm{H}, 0,8.3194471587,0.2594380964,-1.9400751198$ $\mathrm{H}, 0,9.3275029675,-1.763447682,-0.8914181483$ $\mathrm{H}, 0,7.972941001,-3.1873685933,0.5993716476$ $\mathrm{H}, 0,5.8522317369,-3.6931321149,1.6960443737$ $\mathrm{H}, \mathrm{O}, 4.0858689472,-5.1297215458,2.0875532648$ $\mathrm{H}, \mathrm{O}, 2.9224877673,-4.8598610842,3.4096427442$ $\mathrm{H}, 0,4.5762753525,-4.2085916604,3.5412541612$

\section{INT-8- (R)}

Opt a wB97XD/SDD/6-31G $(\mathrm{d}, \mathrm{p})$ in toluene (SMD model)

SCF Done: $\mathrm{E}(\mathrm{RwB} 97 \mathrm{XD})=-3069.33692686 \mathrm{a} \cdot \mathrm{u}$.

Zero-point correction $=0.849642$ Hartree/Particle

Sum of electronic and thermal Free Energies $=-3068.599451 \mathrm{a} . \mathrm{u}$. d $358.15 \mathrm{~K}$ Sp a B3LYP-D3 (BJ)/def2-TZVPP in toluene (SMD model)

$\mathrm{SCF}$ Done: E (RB3LYP) $=-3071.38779751 \mathrm{a} \cdot \mathrm{u}$.

$\mathrm{Rh}, 0,-0.7493968999,-0.7981784584,-0.706125409$

$0,0,-1.850787932,0.2854936224,-2.2194651115$

$\mathrm{C}, 0,-3.0161493833,0.546194133,-1.8988767398$

$C, 0,-3.3240725482,0.7025051,-0.428075594$

C, $0,-4.0748711903,0.7054444676,-2.8813844083$

$C, 0,-4.7898327066,0.7916439983,-0.083255437$

$C, 0,-5.3630414439,0.7844643549,-2.492393529$

C, $0,-5.7618638826,0.8157183704,-1.0956633702$

$\mathrm{H}, 0,-6.1463778244,0.837057059,-3.2435817207$

C, $0,-7.5099252446,1.0067522815,0.5648881449$

C, $0,-6.5403389103,1.0183278856,1.5684777303$

C, $0,-5.1934379556,0.920538065,1.2440209823$

C $, 0,-7.1204867772,0.9159345537,-0.7614554991$

$\mathrm{H}, 0,-8.5619408298,1.0804887912,0.8198499558$

$\mathrm{H}, 0,-6.8367075521,1.1077361114,2.6086688714$

$\mathrm{H}, 0,-4.4432121601,0.9468062648,2.0272885017$

$\mathrm{H}, 0,-7.8630551186,0.9286971783,-1.5542503734$

C, $0,-2.6000038885,2.0027299824,-0.085635054$

C, $0,-2.9323415997,3.2862260197,-0.4982150961$

$C, 0,-1.4549569665,1.7368183405,0.667977238$

C, $0,-2.0975198573,4.3410875179,-0.134078718$

$\mathrm{H}, \mathrm{O},-3.8335343476,3.4662549145,-1.0781683304$

C, $0,-0.5952476415,2.7930670309,0.9811056153$

C, $0,-0.9314089776,4.0855719234,0.5909968955$

$\mathrm{H}, \mathrm{O},-2.3488790725,5.3570555731,-0.4218038905$

$\mathrm{H}, 0,0.3227303156,2.6102748568,1.5297819682$

$\mathrm{H}, \mathrm{O},-0.2698167838,4.9058941465,0.8536938294$

C, $0,-2.4817986245,-0.3913162005,0.3666530709$

C, $0,-1.3819860116,0.3037057464,1.0401679198$

C, $0,-0.9108100506,-0.0260670198,2.4301730286$

C, $0,-0.9487661187,0.9490917611,3.4394512181$

C, $0,-0.5292152024,-1.3159078687,2.8169734966$

C, $0,-0.5907922245,0.655065,4.7528298856$

$\mathrm{H}, \mathrm{O},-1.276396737,1.9558062763,3.2078843447$

C, $0,-0.1758122495,-1.6179307483,4.1260026824$

$\mathrm{H}, \mathrm{O},-0.5356070912,-2.1015604729,2.0793809574$

C, $0,-0.1938167183,-0.6296142695,5.1058758663$

$\mathrm{H}, 0,-0.6297694451,1.4401955487,5.502291915$

$\mathrm{H}, 0,0.1140383799,-2.633927609,4.3780047929$

$\mathrm{H}, 0,0.0894897838,-0.8593057786,6.1284156067$ 
$C, 0,-3.2635766737,-1.566119131,0.8702000357$

$\mathrm{C}, 0,-3.6055303421,-1.737048524,2.215662438$

$\mathrm{C}, 0,-3.7411417136,-2.5058029282,-0.0528074129$

$\mathrm{C}, 0,-4.3698531812,-2.8263228424,2.6273356084$

$\mathrm{H}, 0,-3.272749668,-1.014402388,2.9519874299$

C, $0,-4.5169727547,-3.5854403515,0.3517557989$

$\mathrm{H}, \mathrm{O},-3.4824618015,-2.3878591062,-1.1018508222$

$\mathrm{C}, 0,-4.8291700113,-3.7550768862,1.6994796089$

$\mathrm{H}, 0,-4.6132414872,-2.940417151,3.6796274185$

$\mathrm{H}, \mathrm{O},-4.8736198949,-4.2991981261,-0.3850849539$

$\mathrm{H}, 0,-5.4273865306,-4.6022442778,2.0210689171$

$\mathrm{Cl}, 0,-3.6320063909,0.6724011212,-4.5637638707$

$0,0,0.9877251608,1.8682446832,-2.7259232067$

$0,0,3.5796123186,-3.671783416,1.3091078848$

$\mathrm{C}, 0,0.1518045048,-2.7217872705,-0.1615415527$

C, $0,-0.3885519631,-2.912556393,-1.4659965892$

$\mathrm{C}, 0,0.2676424416,-1.9780196505,-2.2983639713$

C, $0,1.328153872,-1.3237708011,-1.5677976921$

C, $0,1.2464247619,-1.7702729823,-0.2322797152$

$\mathrm{C}, 0,2.3545062992,-0.4127180213,-2.1872934043$

C, $0,2.602899068,0.8380116926,-1.3805192941$

C, $0,1.8476775303,2.0108735746,-1.6919205765$

C, $0,2.0234255759,3.1697909913,-0.9813801726$

C, $0,2.9212804525,3.2039333465,0.1172904366$

C, $0,3.0753285365,4.3742361443,0.9051105645$

C, $0,3.9139915343,4.387633045,1.9917595914$

C, $0,4.6398223747,3.2279504577,2.3414662518$

C, $0,4.5160690486,2.0833631265,1.592755724$

C, 0,3.6610956806,2.040903815,0.4618115181

$\mathrm{C}, 0,3.495725895,0.8595517906,-0.3335381498$

C, $0,0.046217123,2.8948006452,-2.9572803885$

$\mathrm{C}, 0,2.1490216844,-1.3774792048,0.9153775317$

C, $0,3.6136140522,-1.4540636847,0.5497890494$

$\mathrm{C}, 0,4.2656676233,-0.3826091391,-0.0186818731$

$\mathrm{C}, 0,5.6531903526,-0.4716593911,-0.3725374224$

$\mathrm{C}, 0,6.3543531431,0.6004283347,-0.9833530184$

C, $0,7.6822950671,0.4796364045,-1.3103890525$

$\mathrm{C}, 0,8.372681331,-0.7241429024,-1.0481717633$

$\mathrm{C}, 0,7.717460152,-1.7821188519,-0.4702040956$

C, $0,6.3451841293,-1.6864627417,-0.121567101$

$\mathrm{C}, 0,5.6549556273,-2.784887909,0.4562414057$

$\mathrm{C}, 0,4.3249638337,-2.6742260504,0.7728866026$

C, $0,4.2068808629,-4.9121108173,1.5529553371$

$\mathrm{H}, \mathrm{O},-0.0991277355,-3.3189555418,0.703693338$

$\mathrm{H}, 0,-1.1901639927,-3.5827156572,-1.7409166252$

$\mathrm{H}, 0,0.0483766494,-1.7905538651,-3.342693006$

$\mathrm{H}, \mathrm{0}, 2.0144103471,-0.1391693008,-3.1869081319$

$\mathrm{H}, 0,3.2933474903,-0.9650941771,-2.3042393285$

$\mathrm{H}, 0,1.4538210567,4.0617144884,-1.2139372354$

$\mathrm{H}, 0,2.5131366067,5.2639843107,0.6326647481$

$\mathrm{H}, 0,4.0186994619,5.2893692224,2.5877058211$

$\mathrm{H}, 0,5.2955270636,3.241990983,3.206472225$

$\mathrm{H}, 0,5.0727207668,1.192839602,1.8660547855$

$\mathrm{H}, \mathrm{O},-0.5542070027,3.0915891103,-2.0630350032$

$\mathrm{H}, \mathrm{O},-0.6027343695,2.5357918482,-3.7558586708$

$\mathrm{H}, \mathrm{O}, 0.534482626,3.8226306499,-3.2833619554$

$\mathrm{H}, 0,1.9514817334,-2.0339982186,1.7625449702$

$\mathrm{H}, 0,1.91312404,-0.3610655953,1.2383996935$ 
$\mathrm{H}, 0,5.8264081161,1.5249300526,-1.1917412407$

$\mathrm{H}, 0,8.20387775,1.3107992407,-1.7746635564$

$\mathrm{H}, 0,9.4229735644,-0.8115925202,-1.3096253459$

$\mathrm{H}, 0,8.2422281751,-2.713269097,-0.2727990482$

$\mathrm{H}, 0,6.2025903577,-3.7044468921,0.6288440552$

$\mathrm{H}, 0,4.5909370712,-5.3578949177,0.6271553153$

$\mathrm{H}, 0,3.4381648883,-5.5641729333,1.9696980563$

$\mathrm{H}, 0,5.02599244,-4.815848555,2.2766667687$

\section{INT-9- $\left(S_{\mathrm{a}}\right)$}

Opt a wB97XD/SDD/6-31G $(\mathrm{d}, \mathrm{p})$ in toluene (SMD model)

SCF Done: $\mathrm{E}(\mathrm{RwB} 97 \mathrm{XD})=-3069.31355722 \mathrm{a} \cdot \mathrm{u}$.

Zero-point correction $=0.848982$ Hartree/Particle

Sum of electronic and thermal Free Energies $=-3068.577883 \mathrm{a} . \mathrm{u}$. d $358.15 \mathrm{~K}$

Sp a B3LYP-D3 (BJ)/def2-TZVPP in toluene (SMD model)

SCF Done: E (RB3LYP) $=-3071.37509029$ a.u.

$\mathrm{Rh}, 0,-1.4853678458,-1.031811603,0.6432826756$

$0,0,-0.3588520308,-1.2789995861,2.557013575$

C, $0,-0.2276112646,-0.0829255771,2.1327815564$

C, $0,0.3473574476,0.1322714997,0.8005528562$

$C, 0,-0.6454462506,1.0853674687,2.9171980503$

C, 0, 0.1659994936,1.4799720596,0.2158994652

C, $0,-0.6867407441,2.3179374585,2.3765890051$

C, $0,-0.3638760536,2.5365383527,0.9845597272$

$\mathrm{H}, 0,-1.0018812801,3.1657593344,2.9773509235$

C, $0,-0.2611290684,4.0076519983,-0.9381009304$

C, $0,0.2905371433,2.9652413743,-1.6954348177$

C, $0,0.5105790285,1.7253556202,-1.1220633778$

C, $0,-0.5747692144,3.7947807456,0.3911559297$

$\mathrm{H}, \mathrm{O},-0.4327554636,4.9787215324,-1.3911320805$

$\mathrm{H}, 0,0.5469144299,3.1295037241,-2.7374991357$

$\mathrm{H}, 0,0.9351843507,0.9157820617,-1.7079468708$

$\mathrm{H}, \mathrm{O},-0.9902773699,4.5991553332,0.9921837937$

C, $0,1.7064143443,-0.4683431878,0.5840781503$

C, $0,2.7715294344,0.4130373827,0.8316175061$

C, $0,1.9860580242,-1.8096073045,0.2714661553$

C, $0,4.094054954,0.0014555958,0.8048616199$

$\mathrm{H}, 0,2.5469916425,1.4478297527,1.0713398751$

C, $0,3.3374021473,-2.2100789609,0.2656251074$

C, $0,4.375710741,-1.3322505808,0.5285439693$

$\mathrm{H}, 0,4.8896099036,0.7102509407,1.0122003054$

$\mathrm{H}, 0,3.5760167237,-3.2433084959,0.04628382$

$\mathrm{H}, \mathrm{O}, 5.400487768,-1.6908115048,0.5145965991$

C, $0,-0.3886284095,-2.6186743233,-0.0510521676$

C, $0,0.9489772046,-2.7994889412,-0.0939523877$

C, $0,-1.2522021414,-3.7441826644,-0.5156865715$

C, $0,-1.9424957147,-4.5418192161,0.4046940381$

C, $0,-1.4286201523,-4.0004843534,-1.8795005802$

C, $0,-2.7828106647,-5.5632070891,-0.0236314131$

$\mathrm{H}, \mathrm{O},-1.8499107859,-4.3190750909,1.4618135178$

$\mathrm{C}, 0,-2.2761222068,-5.0180008445,-2.3108112247$

$\mathrm{H}, 0,-0.8960139952,-3.3926452229,-2.6051098027$

C, $0,-2.9575850526,-5.8047342309,-1.385804459$

$\mathrm{H}, \mathrm{O},-3.3088121145,-6.1686951695,0.7091832523$

$\mathrm{H}, \mathrm{O},-2.4000258217,-5.1990261942,-3.3747013301$

$\mathrm{H}, \mathrm{O},-3.6175563891,-6.5986679347,-1.7217070338$ 
$C, 0,1.4731867073,-4.1270039794,-0.5731927943$ $C, 0,1.4902119448,-5.235092821,0.2765386614$ C, $0,1.9532393848,-4.2766199192,-1.8779388389$ C, $0,1.9611170763,-6.4670228677,-0.1695585938$ $\mathrm{H}, \mathrm{O}, 1.1250908316,-5.1253721592,1.2933294427$ C, $0,2.4218668317,-5.5067401523,-2.3281844114$ $\mathrm{H}, 0,1.9564264707,-3.4172177701,-2.5429585494$ C, $0,2.4257336505,-6.607600574,-1.4743412438$ $\mathrm{H}, 0,1.9633222895,-7.3194136293,0.5034760124$ $\mathrm{H}, \mathrm{O}, 2.7831800539,-5.6065543048,-3.3475762922$ $\mathrm{H}, 0,2.7895079412,-7.5690097522,-1.8243442099$ $\mathrm{Cl}, 0,-1.0637149028,0.8125205694,4.5808395642$ $0,0,-2.6267165787,-3.329544726,3.5488114003$ $0,0,-5.5813429152,2.6437368371,0.5287013395$ C, $0,-2.8441176534,0.5004513279,-0.2579669589$ C, $0,-2.7493702708,-0.6926858384,-1.0481307464$ $C, 0,-3.3819525976,-1.7355052574,-0.2935440093$ $C, 0,-3.7679967084,-1.2205380725,0.9622305544$ C, $0,-3.4505292366,0.1905812458,0.9782779743$ C, $0,-4.4976151188,-2.0234752264,2.0075260079$ C, $0,-4.2476983267,-1.6268673075,3.4463275959$ C, $0,-3.2732167553,-2.3410147215,4.2101396511$ C, $0,-3.0446498382,-2.0248897582,5.5255533748$ C, $0,-3.7516127794,-0.9642892488,6.1443172578$ C, $0,-3.5057746943,-0.6067989249,7.4961089407$ C, $0,-4.1722208354,0.4406735939,8.078738077$ C, $0,-5.1164215172,1.1830884485,7.3346465085$ $C, 0,-5.3738316055,0.8601669514,6.0259455459$ C, $0,-4.7061357505,-0.2240541425,5.3980260899$ C, $0,-4.9387033827,-0.5876358551,4.0324456169$ C, $0,-1.4749872529,-3.879495919,4.1611859$ C, $0,-3.8509305659,1.1765176291,2.0428917329$ C, $0,-5.3443626916,1.0906893399,2.2664661545$ $C, 0,-5.8748498215,0.2283237576,3.2001172492$ $C, 0,-7.2938431674,0.0994578789,3.3525396402$ C, $0,-7.8813738704,-0.7952810049,4.2845958136$ C, $0,-9.2456407176,-0.8933847887,4.4018079249$ $C, 0,-10.0882503236,-0.1025696804,3.5894653763$ C, $0,-9.5482980174,0.764627162,2.6737818281$ C, $0,-8.1422230762,0.8858133691,2.5277237535$ C, $0,-7.57697005,1.7666877506,1.5677099136$ $\mathrm{C}, 0,-6.2152848833,1.8563393505,1.4323790771$ C, $0,-6.3750549011,3.4073154659,-0.3554884025$ $\mathrm{H}, \mathrm{O},-2.4780996448,1.4772821669,-0.5444458312$ $\mathrm{H}, \mathrm{O},-2.3829745756,-0.7633205258,-2.0623031408$ $\mathrm{H}, \mathrm{O},-3.5324812281,-2.7535017456,-0.6158427548$ $\mathrm{H}, \mathrm{O},-4.2365250915,-3.0737888346,1.8642631038$ $\mathrm{H}, 0,-5.5693524269,-1.9271405078,1.7974161973$ $\mathrm{H}, 0,-2.3051526195,-2.5602975351,6.108943199$ $\mathrm{H}, 0,-2.7723041806,-1.1759264234,8.0609553401$ $\mathrm{H}, 0,-3.9725804501,0.7049975367,9.1127923378$ $\mathrm{H}, \mathrm{O},-5.6366983259,2.0142779361,7.8002347994$ $\mathrm{H}, \mathrm{O},-6.0966434028,1.4369637416,5.4588580386$ $\mathrm{H}, 0,-0.7565047959,-3.0935151324,4.4121373977$ $\mathrm{H}, 0,-1.0261201554,-4.5502262957,3.4276906969$ $\mathrm{H}, 0,-1.7353020363,-4.459541783,5.055305817$ $\mathrm{H}, \mathrm{O},-3.5705268291,2.1795136838,1.7192378448$ $\mathrm{H}, \mathrm{O},-3.3326074177,0.969600256,2.9797875401$ 
$\mathrm{H}, 0,-7.2363657475,-1.404008043,4.9099771849$

$\mathrm{H}, 0,-9.680674934,-1.5812971596,5.119971962$

$\mathrm{H}, \mathrm{O},-11.166315159,-0.1851170028,3.6892891099$

$\mathrm{H}, \mathrm{O},-10.1925297104,1.3712758712,2.0429313121$

$\mathrm{H}, \mathrm{O},-8.2462068613,2.3482684876,0.9439479301$

$\mathrm{H}, 0,-7.0169945172,2.7669618485,-0.9724788758$

$\mathrm{H}, 0,-5.6793254027,3.9444863277,-1.0011629924$

$\mathrm{H}, \mathrm{O},-6.9962151701,4.1327645175,0.1842808538$

\section{TS-8- $\left(S_{\mathrm{a}}\right)$}

Opt a wB97XD/SDD/6-31G $(\mathrm{d}, \mathrm{p})$ in toluene (SMD model)

SCF Done: $\mathrm{E}(\mathrm{RwB} 97 \mathrm{XD})=-3069.27002250 \mathrm{a} \cdot \mathrm{u}$.

Zero-point correction $=0.847911$ Hartree/Particle

Sum of electronic and thermal Free Energies $=-3068.535003 \mathrm{a} . \mathrm{u}$. d $358.15 \mathrm{~K}$

Imaginary Freq. $=-257.6528 \mathrm{~cm}^{-1}$

Sp a B3LYP-D3 (BJ)/def2-TZVPP in toluene (SMD model)

SCF Done: $\mathrm{E}(\mathrm{RB} 3 \mathrm{LYP})=-3071.33259087 \mathrm{a} \cdot \mathrm{u}$.

$\mathrm{Rh}, 0,0.6302481309,-0.0387913601,-1.4262265021$

$0,0,1.2876547742,-1.4492040663,0.0359724003$

C, $0,2.2928852378,-0.9022872591,-0.5902955752$

C, $0,2.5993822384,-1.4646624725,-1.9197268274$

C, $0,3.2609607223,0.0122504932,0.0219601398$

$C, 0,3.8747284411,-1.0600560585,-2.5595824304$

C, $0,4.3691431618,0.4183284155,-0.6255803013$

$\mathrm{C}, 0,4.6936473822,-0.0944252416,-1.9389364907$

$\mathrm{H}, 0,5.059045244,1.1101708103,-0.1539470099$

C, $0,6.2386202324,-0.2110142672,-3.8061465822$

C, $0,5.4541547318,-1.2118174485,-4.391605953$

C, $0,4.2937175138,-1.6331826112,-3.7690136814$

C, $0,5.8683716591,0.3276872601,-2.5884167255$

$\mathrm{H}, 0,7.1450960894,0.128462082,-4.2971718056$

$\mathrm{H}, 0,5.7525567905,-1.6567193626,-5.3353825514$

$\mathrm{H}, 0,3.6860774981,-2.4043077909,-4.2296252674$

$\mathrm{H}, \mathrm{O}, 6.4880854106,1.0828828197,-2.1131245392$

C, $0,2.2463899007,-2.9181996647,-2.0008083394$

$\mathrm{C}, 0,2.775182364,-3.8492809155,-1.1088670224$

C, $0,1.4119133701,-3.3276055606,-3.0387355096$

C, $0,2.5104405465,-5.2003695598,-1.2963976351$

$\mathrm{H}, \mathrm{O}, 3.4094746095,-3.5220089112,-0.2908581988$

C, $0,1.1717115273,-4.6911954066,-3.2434022453$

$\mathrm{C}, 0,1.7178244313,-5.6198889894,-2.3688365498$

$\mathrm{H}, 0,2.9380525427,-5.93210941,-0.6182900067$

$\mathrm{H}, 0,0.5569662258,-5.0175384554,-4.0760559539$

$\mathrm{H}, 0,1.5276889093,-6.6782428283,-2.5188960501$

C, $0,1.1582740051,-0.9639043364,-3.3772420059$

$\mathrm{C}, 0,0.9831788824,-2.2143773454,-3.8791700813$

$0,0,-1.865726605,-2.8574395394,-0.8315035077$

$0,0,-1.0723111224,3.8708966613,1.9641175074$

C, $0,0.2930308251,2.17486349,-0.8563367026$

C, $0,-0.0956919922,1.9178538542,-2.1942283033$

C, $0,-1.2091796263,0.998384965,-2.1735977493$

C, $0,-1.4338239172,0.6068364066,-0.8455838085$

C, $0,-0.4549017486,1.3166896597,-0.0177789411$

C, $0,-2.5748744966,-0.2587175999,-0.3735968874$

C, $0,-2.2521163289,-1.191589296,0.7724256422$

C, $0,-1.8635443225,-2.5353848611,0.4820605043$ 
C, $0,-1.5442932498,-3.4098945672,1.4898910925$ $C, 0,-1.584747581,-2.9892648937,2.8431819795$ C, $0,-1.2454983588,-3.8743765847,3.8998126292$ C, $0,-1.2796119042,-3.4570674514,5.2060752316$ C, $0,-1.6503457249,-2.1299793058,5.5177047354$ C, $0,-1.9843258338,-1.2528405513,4.5161716791$ C, $0,-1.9702248783,-1.6591693232,3.1561961516$ C, $0,-2.3101734525,-0.7728654509,2.0832599176$ C, $0,-1.4182667461,-4.1473620202,-1.1879441649$ C, $0,-0.3849192521,1.2704862853,1.4850743526$ C, $0,-1.7240755125,1.6233613881,2.0910788467$ $C, 0,-2.6545398316,0.6505871073,2.3780474386$ C, $0,-3.9376513925,0.9971665991,2.9145850648$ C, $0,-4.9274367144,0.0245309011,3.2123244422$ C, $0,-6.1428528592,0.3927341677,3.7336419119$ C, $0,-6.4285686699,1.754158895,3.9784530903$ C, $0,-5.4949349036,2.7174543176,3.6901467833$ $C, 0,-4.2300657092,2.3670556627,3.1505815645$ $C, 0,-3.2639578669,3.3593771401,2.835712828$ C, $0,-2.0488942143,2.9976500513,2.3118203662$ C, $0,-1.3269150499,5.2501089533,2.1237704921$ $\mathrm{H}, 0,1.0855628997,2.8349128948,-0.5305309846$ $\mathrm{H}, 0,0.299834911,2.4031809203,-3.074679237$ $\mathrm{H}, \mathrm{O},-1.7550346721,0.6436319928,-3.0363293384$ $\mathrm{H}, 0,-2.9339967107,-0.8388186072,-1.225124613$ $\mathrm{H}, 0,-3.3895493811,0.4077752332,-0.0681305499$ $\mathrm{H}, 0,-1.2406523609,-4.427981691,1.2770082617$ $\mathrm{H}, 0,-0.9521357173,-4.8915220146,3.653662906$ $\mathrm{H}, \mathrm{O},-1.0162332905,-4.1447862791,6.0041804555$ $\mathrm{H}, \mathrm{0},-1.6678247815,-1.802851342,6.5526904141$ $\mathrm{H}, \mathrm{O},-2.2627249663,-0.2330720509,4.7602819357$ $\mathrm{H}, \mathrm{O},-0.3879619143,-4.315477562,-0.8578298492$ $\mathrm{H}, 0,-1.458058626,-4.1916792251,-2.2759561123$ $\mathrm{H}, \mathrm{O},-2.0728788303,-4.9262570228,-0.7748211162$ $\mathrm{H}, 0,0.3750948154,1.9763628591,1.8201177423$ $\mathrm{H}, 0,-0.0754256359,0.2741907918,1.8074593003$ $\mathrm{H}, \mathrm{0},-4.7102217218,-1.022009845,3.025331242$ $\mathrm{H}, 0,-6.8877022587,-0.3639243587,3.9594038122$ $\mathrm{H}, 0,-7.3915439219,2.0369109752,4.3929083514$ $\mathrm{H}, \mathrm{O},-5.712360398,3.7666386043,3.8723164513$ $\mathrm{H}, 0,-3.5157710437,4.398577625,3.0140887102$ $\mathrm{H}, 0,-2.1828805589,5.5732059277,1.5185425751$ $\mathrm{H}, 0,-0.4301086082,5.766042743,1.7786069006$ $\mathrm{H}, 0,-1.5069236281,5.509139486,3.1745297313$ $\mathrm{Cl}, 0,2.9572713562,0.5322617416,1.6528506562$ $\mathrm{C}, 0,1.1876407129,0.2068479008,-4.3056900787$ C, $0,2.2767995898,1.0847228124,-4.3786206382$ C, $0,0.0842087339,0.4604887882,-5.134297129$ C, $0,2.2613378507,2.1719176774,-5.2469894477$ $\mathrm{H}, 0,3.1388677628,0.934092591,-3.7433424036$ C, $0,0.0690275493,1.5486756728,-5.9988296266$ $\mathrm{H}, \mathrm{O},-0.7739545992,-0.201418923,-5.0946507834$ $\mathrm{C}, 0,1.1584280593,2.4140487115,-6.0608872538$ $\mathrm{H}, 0,3.1209414431,2.8347828651,-5.281243996$ $\mathrm{H}, \mathrm{O},-0.8011250121,1.7197959136,-6.6255065511$ $\mathrm{H}, 0,1.1477083136,3.2649909991,-6.7348219281$ $C, 0,0.6032825394,-2.5112745796,-5.2914087649$ C, $0,-0.6345288363,-3.0968719308,-5.5730376872$ 
C, $0,1.4760761424,-2.2341405489,-6.3478876688$

C, $0,-0.9934819859,-3.3998518821,-6.8841142022$

$\mathrm{H}, \mathrm{O},-1.3241549946,-3.301195279,-4.7583182188$

C, $0,1.1159016143,-2.533198709,-7.6571964812$

$\mathrm{H}, 0,2.4305296798,-1.7600580945,-6.140170089$

C, $0,-0.1185149153,-3.1186792556,-7.9297277502$

$\mathrm{H}, \mathrm{O},-1.9598442564,-3.8511042947,-7.0878791953$

$\mathrm{H}, \mathrm{O}, 1.8008436004,-2.3052615545,-8.4682631153$

$\mathrm{H}, \mathrm{O},-0.3978228909,-3.3510482361,-8.9527828618$

\section{TS-9- $\left(S_{a}\right)$}

Opt \& wB97XD/SDD/6-31G $(\mathrm{d}, \mathrm{p})$ in toluene (SMD model)

$\mathrm{SCF}$ Done: $\mathrm{E}(\mathrm{RwB} 97 \mathrm{XD})=-3069.27978036 \mathrm{a} \cdot \mathrm{u}$.

Zero-point correction $=0.847356$ Hartree/Particle

Sum of electronic and thermal Free Energies $=-3068.545215 \mathrm{a} \cdot \mathrm{u}$. d $358.15 \mathrm{~K}$

Imaginary Freq. $=-232.8724 \mathrm{~cm}^{-1}$

Sp \& B3LYP-D3 (BJ)/def2-TZVPP in toluene (SMD model)

SCF Done: E (RB3LYP) $=-3071.34585334$ a.u.

$\mathrm{Rh}, 0,4.6371394071,-0.3228390383,3.5232024091$

$0,0,2.4178302077,-0.2246375052,3.9945583506$

C, $0,1.5288681069,-0.2340658315,4.9586037709$

C, $0,1.4721596122,-1.2272135377,5.9414381284$

C, $0,0.5791127178,0.8400251906,5.0387447497$

C, $0,0.5799717727,-1.1072797148,7.0514024286$

C, $0,-0.3201479222,0.9444717614,6.0588547373$

C, $0,-0.3283745947,-0.0109597853,7.1057216174$

$\mathrm{H}, 0,-1.0190605171,1.7745877129,6.0869435459$

$C, 0,-1.2000058666,-0.78586317,9.2350917141$

C, $0,-0.2773606255,-1.8557874266,9.2072989015$

C, $0,0.5845162273,-2.0129558577,8.1508130301$

C, $0,-1.2158979739,0.1182678031,8.2043317666$

$\mathrm{H}, 0,-1.8809302946,-0.6768529605,10.0733880291$

$\mathrm{H}, 0,-0.2456929221,-2.5590680729,10.0343057614$

$\mathrm{H}, \mathrm{O}, 1.2937393662,-2.8333945326,8.1570861102$

$\mathrm{H}, \mathrm{O},-1.9064235624,0.9576937259,8.2168791637$

C, $0,2.3160747953,-2.4337998181,5.7379865817$

$C, 0,1.6949525031,-3.6929134173,5.7328828235$

C, $0,3.6908887966,-2.3608000994,5.4582201132$

C, $0,2.4000231877,-4.8586335476,5.4730292344$

$\mathrm{H}, 0,0.6279823479,-3.7435833777,5.9285679835$

C, $0,4.3877502908,-3.5452944803,5.1709337368$

C, $0,3.7608044842,-4.7811040721,5.17948024$

$\mathrm{H}, \mathrm{O}, 1.889607553,-5.8167077825,5.4815521928$

$\mathrm{H}, \mathrm{O}, 5.4475714048,-3.478083102,4.9426982884$

$\mathrm{H}, 0,4.329000134,-5.6785810523,4.9548964891$

C, $0,4.0833368804,0.2243127947,5.2320895624$

$C, 0,4.5033440755,-1.1050338846,5.5303908357$

$\mathrm{Cl}, 0,0.5926066939,2.0494207623,3.7795730833$

C, $0,5.6500614961,-1.1258661715,6.5091040771$

$C, 0,5.611299922,-1.9597550761,7.6358285582$

$C, 0,6.7364553249,-0.250870087,6.375509384$

C, $0,6.6276825837,-1.925872882,8.5839849105$

$\mathrm{H}, 0,4.7753831313,-2.6373413128,7.773634168$

$\mathrm{C}, 0,7.7497879355,-0.2127760944,7.3276807663$

$\mathrm{H}, 0,6.7859057193,0.4066189774,5.5138655066$

C, $0,7.7028234131,-1.0530337413,8.4358733489$ 
$\mathrm{H}, 0,6.5743941242,-2.5823827619,9.4472925317$ $\mathrm{H}, 0,8.5805622899,0.4744698049,7.1983050281$ $\mathrm{H}, 0,8.4959247577,-1.0287193931,9.1766950052$ C, $0,3.7656905578,1.4071171049,5.9818633789$ C, $0,3.7849673249,2.6610731299,5.3532349934$ C, 0,3.3562007126,1.2991674091, 7.3213790876 C, 0,3.4092529856,3.7940988139,6.0584426091 $\mathrm{H}, 0,4.0799219704,2.7169907785,4.3101942559$ C, $0,2.9789777264,2.4350179188,8.0212732202$ $\mathrm{H}, 0,3.3342095013,0.3225120529,7.7933569646$ C, 0,3.0052419529,3.6780785532, 7.3888824853 $\mathrm{H}, 0,3.4160865848,4.7647886903,5.5738437633$ $\mathrm{H}, 0,2.6551824564,2.3540540881,9.0533683634$ $\mathrm{H}, 0,2.7030453572,4.5653580329,7.9370988182$ $0,0,2.1791766763,-2.3101078839,1.5661197899$ $0,0,6.2295654128,3.4969823913,-0.2215248796$ $C, 0,6.1665721686,1.0028222052,2.5961056346$ C, $0,6.6471185264,-0.34601631,2.8128013865$ C, $0,5.9868673604,-1.2081747642,1.8770895635$ C, $0,4.9954677159,-0.4418998549,1.2375845398$ C, $0,5.1232488043,0.9512534439,1.6580704999$ C, $0,4.0663557476,-0.9609152048,0.1707072787$ C, $0,2.6531288195,-0.4330713344,0.2567358201$ C, $0,1.6955032814,-1.1771057029,1.0110714136$ C, $0,0.3992066055,-0.7448269964,1.124755803$ C, $0,-0.0026225123,0.4704651278,0.5146233774$ C, $0,-1.3305340835,0.9535321467,0.6464951119$ C, $0,-1.706694953,2.1448807045,0.0806334363$ C, $0,-0.7685837786,2.9140264153,-0.6434327155$ $C, 0,0.5223124996,2.4709356033,-0.7892018379$ C, $0,0.9380433548,1.2373496529,-0.2237075299$ $C, 0,2.2790999562,0.747307178,-0.3450593307$ C, $0,1.3343949026,-3.0333251355,2.4360030303$ C, 0, 4.3106494007,2.1209769098,1.1608559267 C, 0, 4.282360289,2.1978436681,-0.3494481299 C, $0,3.3094685715,1.5467522713,-1.0754553183$ C, $0,3.3128430125,1.5896908434,-2.5085606103$ C, $0,2.3433786723,0.9119707019,-3.2929557139$ C, $0,2.3767326024,0.9749564611,-4.6637612621$ C, $0,3.3850399871,1.7172800017,-5.3174404642$ C, $0,4.3407475212,2.3755628187,-4.5855405571$ C, $0,4.3329050239,2.3271027068,-3.1671087456$ C, $0,5.329759403,2.9895546834,-2.4028532635$ C, $0,5.3107787196,2.9170421756,-1.0330504852$ $C, 0,7.272764066,4.2337170571,-0.8230719296$ $\mathrm{H}, 0,6.5274123392,1.8938102546,3.093590083$ $\mathrm{H}, 0,7.4720987575,-0.6268316695,3.4544733648$ $\mathrm{H}, 0,6.1501964563,-2.2692451777,1.7500977074$ $\mathrm{H}, \mathrm{O}, 4.0542995644,-2.0503727169,0.2245248962$ $\mathrm{H}, 0,4.4942925406,-0.6851973133,-0.7990753531$ $\mathrm{H}, 0,-0.3311986501,-1.3000503179,1.7015983087$ $\mathrm{H}, 0,-2.0435523838,0.365060269,1.2175116315$ $\mathrm{H}, 0,-2.7252522048,2.503720504,0.1943539872$ $\mathrm{H}, 0,-1.0701059306,3.8602041548,-1.082095116$ $\mathrm{H}, 0,1.2405682295,3.0677069527,-1.3415989683$ $\mathrm{H}, 0,0.9638480972,-2.3950985082,3.2434154808$ $\mathrm{H}, \mathrm{O}, 1.9480396351,-3.8277338443,2.8600165539$ $\mathrm{H}, 0,0.4885073456,-3.4753082766,1.8928647682$ 
$\mathrm{H}, 0,4.7418684593,3.0365391909,1.5709113236$

$\mathrm{H}, 0,3.2861809856,2.0450939069,1.53250796$

$\mathrm{H}, 0,1.5681698298,0.3397636587,-2.7944022115$

$\mathrm{H}, 0,1.6269120634,0.4518203561,-5.2488671396$

$\mathrm{H}, 0,3.4037049252,1.7625817779,-6.4021776417$

$\mathrm{H}, 0,5.1217561014,2.9434878149,-5.0843281537$

$\mathrm{H}, 0,6.1018095586,3.5392246606,-2.9290890445$

$\mathrm{H}, 0,7.8880635259,3.6022207106,-1.4756200351$

$\mathrm{H}, 0,7.8894572967,4.610308488,-0.00595338$

$\mathrm{H}, 0,6.8854062701,5.0826135955,-1.4000948146$

\section{$\mathrm{Cu}_{2}(\mathrm{OAC})_{4}$}

Opt d wB97XD/SDD/6-31G $(d, p)$ in toluene (SMD model)

SCF Done: E (RwB97XD) $=-1308.52508838 \mathrm{a} \cdot \mathrm{u}$.

Zero-point correction $=0.211879$ Hartree/Particle

Sum of electronic and thermal Free Energies $=-1308.382225 \mathrm{a} \cdot \mathrm{u}$. d $358.15 \mathrm{~K}$

Sp a B3LYP-D3 (BJ)/def2-TZVPP in toluene (SMD model)

SCF Done: E (RB3LYP) $=-4195.71180167$ a.u.

$\mathrm{Cu}, 0,0.0004633595,-0.0068545534,1.2571486917$

$\mathrm{Cu}, 0,0.0004927879,0.0018479203,-1.2549100564$

C $, 0,-1.7650795568,-1.8239898577,0.0656629521$

$0,0,-1.3544404362,-1.4044174679,1.1885194236$

$0,0,-1.3646989574,-1.4120096711,-1.061486641$

C, $0,-2.841308218,-2.8761153315,0.0722804224$

$\mathrm{H}, \mathrm{O},-3.8093290374,-2.3786020742,-0.0456809932$

$\mathrm{H}, 0,-2.7089762451,-3.5593735416,-0.767743544$

$\mathrm{H}, \mathrm{O},-2.843677824,-3.4222612551,1.0156463865$

C, $0,-1.8229180295,1.7616557753,-0.0601635162$

$0,0,-1.4122650376,1.3608978415,1.0663874412$

$0,0,-1.398316686,1.3551474479,-1.1835231166$

$C, 0,-2.9066440743,2.8061064078,-0.0838272062$

$\mathrm{H}, \mathrm{O},-2.5504980704,3.6820769083,-0.6322688179$

$\mathrm{H}, \mathrm{O},-3.7729643884,2.4124604956,-0.6220971015$

$\mathrm{H}, \mathrm{O},-3.196514378,3.0925971945,0.9263946543$

$\mathrm{C}, 0,1.7647113129,1.8197054343,0.0791899463$

$0,0,1.3537365697,1.3938304405,1.1989614904$

$0,0,1.368513575,1.41039197,-1.0509193337$

C, $0,2.8107545547,2.90202065,0.0839076938$

$\mathrm{H}, 0,3.6948912868,2.5495238324,-0.453735369$

$\mathrm{H}, 0,2.4272561248,3.7741982797,-0.4520589601$

$\mathrm{H}, 0,3.081842625,3.1813980135,1.1013477986$

C, $0,1.8231037978,-1.7658537504,-0.0769408464$

$0,0,1.3997457983,-1.353383516,-1.1970080056$

$0,0,1.4114716483,-1.3709849795,1.0529103737$

$C, 0,2.906003564,-2.8112486693,-0.0800532765$

$\mathrm{H}, 0,3.778606526,-2.4253089105,0.4535491438$

$\mathrm{H}, 0,2.5549583944,-3.6939253842,0.4608657314$

$\mathrm{H}, 0,3.1843720138,-3.0851526492,-1.0970103654$

\section{$\mathrm{Cu}_{2}(\mathrm{OAC})_{2}$}

Opt a wB97XD/SDD/6-31G $(d, p)$ in toluene (SMD model)

SCF Done: E (RwB97XD) $=-851.673250608 \mathrm{a} \cdot \mathrm{u}$.

Zero-point correction $=0.106008$ Hartree/Particle

Sum of electronic and thermal Free Energies $=-851.619423 \mathrm{a} . \mathrm{u}$. d $358.15 \mathrm{~K}$

$\mathrm{Sp}$ a B3LYP-D3(BJ)/def2-TZVPP in toluene (SMD model) 
SCF Done: E (RB3LYP $)=-3738.50290245 \mathrm{a} \cdot \mathrm{u}$.

$\mathrm{Cu}, 0,-0.0196266707,0.0196047712,1.2765745036$

$\mathrm{Cu}, 0,0.0191384559,-0.0161308546,-1.2626917104$

C, $0,-1.7179574016,-1.7675072525,0.0002474754$

$0,0,-1.3135217293,-1.3537747164,1.1277965629$

$0,0,-1.314991578,-1.3517464011,-1.1272616365$

$\mathrm{C}, 0,-2.795624675,-2.8181938773,-0.0006510652$

$\mathrm{H}, \mathrm{O},-3.7669282055,-2.3135669983,-0.0317995893$

$\mathrm{H}, \mathrm{O},-2.7142166939,-3.4518756595,-0.8843286677$

$\mathrm{H}, 0,-2.751104065,-3.4179168813,0.9087052993$

$\mathrm{C}, 0,1.7198887007,1.7686588108,0.0153820908$

$0,0,1.2811724935,1.3890257261,1.1413724426$

$0,0,1.3490400593,1.3208251192,-1.1114742535$

$\mathrm{C}, 0,2.773422074,2.8437384119,-0.001717176$

$\mathrm{H}, 0,3.666404271,2.4632100701,-0.5048964773$

$\mathrm{H}, 0,2.4096256399,3.6945214605,-0.584029958$

$\mathrm{H}, \mathrm{0}, 3.0259833246,3.1662222712,1.0074831593$

\section{INT- 6'-A}

Opt a wB97XD/SDD/6-31G(d,p) in toluene (SMD model)

$\mathrm{SCF}$ Done: $\mathrm{E}(\mathrm{RwB} 97 \mathrm{XD})=-2877.61093466 \mathrm{a} \cdot \mathrm{u}$.

Zero-point correction $=0.793105$ Hartree/Particle

Sum of electronic and thermal Free Energies = -2876.925851 a.u. @ $358.15 \mathrm{~K}$ $\mathrm{Sp}$ a B3LYP-D3 (BJ)/def2-TZVPP in toluene (SMD model)

SCF Done: $\mathrm{E}(\mathrm{RB} 3 \mathrm{LYP})=-2879.52895164 \mathrm{a} \cdot \mathrm{u}$.

$\mathrm{Rh}, 0,-0.8612465934,-1.3374621069,-0.0527189457$

$0,0,-0.3887878968,0.6702029341,-0.037874965$

C, $0,-1.33479268,1.5637758018,0.0417151833$

$\mathrm{C}, 0,-2.5726298728,1.4278540974,-0.596498349$

$\mathrm{C}, 0,-1.1055134599,2.74357881,0.8270556824$

$C, 0,-3.663320484,2.2716430809,-0.2074537776$

$\mathrm{C}, 0,-2.0998782091,3.62967799,1.1086891283$

$\mathrm{C}, 0,-3.4192711853,3.3854996881,0.6480795942$

$\mathrm{H}, 0,-1.9005133326,4.4965234888,1.73064779$

$\mathrm{C}, 0,-5.7846840284,3.9329804858,0.7007287604$

$\mathrm{C}, 0,-6.0456848155,2.8032940216,-0.1050884257$

$C, 0,-5.0210662276,2.0022080653,-0.5469459963$

C, $0,-4.4925668063,4.2090499057,1.0693990052$

$\mathrm{H}, \mathrm{0},-6.6014300818,4.5642231197,1.0367038252$

$\mathrm{H}, 0,-7.0698029097,2.5570721456,-0.3705508927$

$\mathrm{H}, \mathrm{O},-5.2534332933,1.1269614732,-1.1429704164$

$\mathrm{H}, \mathrm{O},-4.2710265077,5.0576733488,1.7118232658$

$\mathrm{C}, 0,-2.6843898865,0.4140631311,-1.6758898288$

$C, 0,-3.4255517101,0.7500214533,-2.8244563056$

$C, 0,-2.0198322741,-0.8310425774,-1.6516371449$

$\mathrm{C}, 0,-3.5874981593,-0.128789961,-3.8865985321$

$\mathrm{H}, \mathrm{O},-3.8765178285,1.7355050947,-2.884489902$

C, $0,-2.1930771861,-1.7065281267,-2.7297991421$

C, $0,-2.9747727437,-1.3755294707,-3.8339747954$

$\mathrm{H}, \mathrm{O},-4.1782416467,0.1648298238,-4.7490984719$

$\mathrm{H}, \mathrm{O},-1.7254536446,-2.6842188712,-2.7133730899$

$\mathrm{H}, \mathrm{O},-3.0911205842,-2.0863851114,-4.647463663$

$\mathrm{Cl}, 0,0.4996539201,3.0131564923,1.461678445$

C, $0,-2.4048083459,-0.9229485167,1.4634887933$

C, $0,-2.91991537,-1.7471609945,0.687633714$ 
$C, 0,-2.3295857678,0.0354301089,2.5432341476$ $C, 0,-1.1350605844,0.2774148938,3.2274850235$ C, $0,-3.4826282578,0.7548090608,2.8867885115$ C, $0,-1.0961506456,1.2150520042,4.2515066814$ $\mathrm{H}, \mathrm{O},-0.2375709394,-0.2514242494,2.930067914$ C, $0,-3.4347058503,1.6948340099,3.9080797705$ $\mathrm{H}, \mathrm{O},-4.4001908136,0.5936697269,2.3307404289$ $\mathrm{C}, 0,-2.2434712556,1.9267940743,4.5924975563$ $\mathrm{H}, 0,-0.1614052629,1.4056487787,4.7688095406$ $\mathrm{H}, \mathrm{O},-4.3274476927,2.2602732515,4.1553829451$ $\mathrm{H}, \mathrm{O},-2.2070551659,2.6695809971,5.3833327846$ $C, 0,-3.9023769259,-2.6654127176,0.0979268252$ C, $0,0.2557773707,-3.0622101018,-0.9130637993$ C, $0,-0.3113891729,-3.4282037151,0.3369636704$ $C, 0,0.3589211395,-2.6643763211,1.3625833991$ C, $0,1.2401478046,-1.7723380858,0.74293195$ C, $0,1.1659363691,-1.9915939771,-0.7018348115$ C, $0,2.0780519215,-0.7288341853,1.4227559468$ C, $0,2.0792528573,-1.4213686276,-1.7586065472$ $\mathrm{H}, \mathrm{O}, 0.0222494876,-3.4981179926,-1.8739188735$ $\mathrm{H}, \mathrm{O},-1.0351368613,-4.2140969282,0.5052254412$ $\mathrm{H}, 0,0.1596034503,-2.7176377666,2.4244439707$ $0,0,0.6563328498,0.3000410333,-3.3109410899$ $0,0,3.6526629308,-2.3808001221,2.9143609479$ C, $0,2.3213305631,0.0697691334,-1.6805691291$ C, $0,1.5315274789,0.934120775,-2.4997297405$ C, $0,1.6992544472,2.2943092581,-2.4560784525$ C, $0,2.6475367065,2.8697707806,-1.5729672288$ C, $0,2.8076333208,4.2766149225,-1.4800112586$ C, $0,3.7085881451,4.8266501151,-0.6042857067$ C, $0,4.4951046431,3.9929306883,0.2209363895$ C, $0,4.3677218529,2.6282058405,0.1453867197$ C, $0,3.4472901714,2.030520776,-0.7540083663$ C, $0,3.2725889923,0.6108816061,-0.8463546739$ C, $0,-0.2032064473,1.0905352724,-4.1042346139$ C, $0,3.5401376664,-0.9133019509,1.0897566056$ C, $0,4.108997465,-0.2893219744,0.0035593242$ C, $0,5.4853939916,-0.5160688726,-0.3308830218$ C, $0,6.103478922,0.0856897175,-1.4577476738$ C, $0,7.4243872533,-0.15048806,-1.7469543867$ C, $0,8.191418569,-1.0036952269,-0.9233670099$ $\mathrm{C}, 0,7.6183115513,-1.607118006,0.1674177482$ $C, 0,6.2543570059,-1.384012369,0.4895546937$ C, $0,5.6490004148,-2.0206560947,1.6055483539$ C, $0,4.3252137672,-1.8016040647,1.8886829578$ C, $0,4.3583629913,-3.2723309367,3.7517444923$ $\mathrm{H}, 0,1.1005451495,2.9549167183,-3.0720112585$ $\mathrm{H}, 0,2.1910928402,4.9134832274,-2.1086068876$ $\mathrm{H}, 0,3.8146426324,5.9053217109,-0.5385282838$ $\mathrm{H}, 0,5.20092752,4.4338468907,0.9180587194$ $\mathrm{H}, 0,4.9714670288,1.9926716728,0.7846760305$ $\mathrm{H}, \mathrm{O},-0.8072593235,1.7637136247,-3.4873260806$ $\mathrm{H}, 0,-0.862860049,0.3942578979,-4.6207141805$ $\mathrm{H}, 0,0.3636500382,1.6743908069,-4.841400425$ $\mathrm{H}, 0,5.5175320818,0.7410535357,-2.0930871167$ $\mathrm{H}, 0,7.8819195854,0.3192762631,-2.6119038116$ $\mathrm{H}, 0,9.236036527,-1.1839480372,-1.1585430044$ $\mathrm{H}, 0,8.2029466876,-2.269262783,0.8006998389$ 
$\mathrm{H}, 0,6.2547942277,-2.6844284217,2.211735473$

$\mathrm{H}, 0,4.742780205,-4.1327655515,3.1902694811$

$\mathrm{H}, 0,3.6412868815,-3.6227299603,4.495274983$

$\mathrm{H}, \mathrm{O}, 5.18995279,-2.7723328411,4.2633058302$

$\mathrm{H}, 0,1.9297585226,-0.8033794842,2.5013894165$

$\mathrm{H}, 0,1.7284567621,0.2569468668,1.0981635176$

$\mathrm{H}, 0,3.0376204439,-1.9462674496,-1.6745129971$

$\mathrm{H}, 0,1.659793835,-1.6684479952,-2.734712945$

$\mathrm{H}, 0,-3.4479884344,-3.626146788,-0.1574552608$

$\mathrm{H}, \mathrm{0},-4.7113773418,-2.8376803351,0.8143402313$

$\mathrm{H}, \mathrm{O},-4.3249839331,-2.2387727026,-0.8149587889$

\section{INT- $6^{\prime}-\mathrm{B}$}

Opt a wB97XD/SDD/6-31G $(\mathrm{d}, \mathrm{p})$ in toluene (SMD model)

SCF Done: $\mathrm{E}(\mathrm{RwB} 97 \mathrm{XD})=-2877.60726246 \mathrm{a} \cdot \mathrm{u}$.

Zero-point correction $=0.793231$ Hartree/Particle

Sum of electronic and thermal Free Energies = -2876.922462 a.u. d $358.15 \mathrm{~K}$

Sp a B3LYP-D3 (BJ)/def2-TZVPP in toluene (SMD model)

SCF Done: E (RB3LYP) $=-2879.52508199$ a.u.

$\mathrm{Rh}, 0,1.0136938933,-1.2975190699,-0.0949200311$

$0,0,0.5236175153,0.6835226017,-0.3849793353$

C, $0,1.4394167141,1.5778465239,-0.6305711026$

$\mathrm{C}, 0,2.7173888332,1.5550869476,-0.0581325155$

$\mathrm{C}, 0,1.1280348378,2.6384317287,-1.5475946211$

$\mathrm{C}, 0,3.7469054857,2.3979046916,-0.5954753913$

C, $0,2.060416386,3.539695251,-1.9645939062$

C, $0,3.4065034956,3.4150005476,-1.5360955038$

$\mathrm{H}, 0,1.7895713789,4.3246385316,-2.6638723451$

C, $0,5.7281808281,4.1040212999,-1.7206692539$

$C, 0,6.0879618645,3.055538539,-0.8460276121$

C, $0,5.1325546095,2.2338934971,-0.3013759241$

$C, 0,4.4106833371,4.2668797194,-2.062483071$

$\mathrm{H}, 0,6.4906406266,4.7563943315,-2.1344279489$

$\mathrm{H}, 0,7.134569511,2.8886115324,-0.6082770837$

$\mathrm{H}, \mathrm{O}, 5.4445843183,1.4239778689,0.3471316943$

$\mathrm{H}, 0,4.1136575904,5.0459293617,-2.7600462069$

C, $0,2.9361485413,0.6772843189,1.1210784925$

C, $0,3.7594747704,1.1582701471,2.1579742043$

$C, 0,2.3086686817,-0.5754227585,1.297331904$

C, $0,4.0447480755,0.4073141925,3.2898789399$

$\mathrm{H}, 0,4.1763604666,2.1567862769,2.0741031197$

C, $0,2.6104782495,-1.3213377326,2.4427710006$

C, $0,3.4769497434,-0.8541484907,3.4265924838$

$\mathrm{H}, 0,4.6969632065,0.8114433258,4.0583457231$

$\mathrm{H}, 0,2.1866156695,-2.3104960071,2.5684847373$

$\mathrm{H}, 0,3.6959597819,-1.4713935285,4.2934256673$

$\mathrm{Cl}, 0,-0.5000405547,2.731200626,-2.1796731063$

C, $0,2.3172591346,-1.0739753808,-1.8593066542$

C, $0,2.9969363668,-1.7685737063,-1.0890688618$

C, $0,1.9392001016,-0.3154841743,-3.062479209$

$C, 0,4.0290095725,-2.6277230769,-0.55313243$

C, $0,5.003551894,-2.1352809278,0.3234971996$

C, $0,4.0640535164,-3.9755677376,-0.9373913282$

C, $0,5.9926741182,-2.9834581336,0.8076883131$

$\mathrm{H}, \mathrm{O}, 4.9739841513,-1.0946353203,0.6250450177$

C, $0,5.0571947683,-4.8166086028,-0.4495097501$ 
$\mathrm{H}, 0,3.3136822993,-4.3524753538,-1.6258503629$

$\mathrm{C}, 0,6.0215741516,-4.3227084291,0.4265310029$

$\mathrm{H}, 0,6.7428802048,-2.593937522,1.4884059628$

$\mathrm{H}, 0,5.0790243525,-5.8580162616,-0.7549164674$ $\mathrm{H}, 0,6.7959942302,-4.9805528227,0.808648735$ C, $0,-0.0043789985,-2.8679284696,1.1210600645$ $\mathrm{C}, 0,0.4749711401,-3.4267199934,-0.0923097464$ C, $0,-0.279945662,-2.8330500175,-1.1731934427$ C, $0,-1.1254557296,-1.8555324154,-0.6397509969$ C, $0,-0.9408869553,-1.8445004453,0.8118650401$ C, $0,-2.0113293728,-0.9193405539,-1.4094498707$ $\mathrm{C}, 0,-1.7840832629,-1.1168408679,1.8285580735$ $\mathrm{H}, 0,0.3107777676,-3.1396261475,2.1184181221$ $\mathrm{H}, 0,1.2039478261,-4.2204756745,-0.1819811219$ $\mathrm{H}, \mathrm{O},-0.1561388651,-3.0478848686,-2.2263968972$ $0,0,-0.2467589781,0.7997261731,2.9879900696$ $0,0,-3.6869088511,-2.7673038316,-2.5091436815$ C, $0,-2.0326406074,0.3472347648,1.5428842604$ C, $0,-1.1820806673,1.3152244716,2.1601369249$ C, $0,-1.3508835179,2.6549845891,1.9235134847$ C, $0,-2.3640409211,3.1021840938,1.03823219$ C, $0,-2.5277404135,4.4810538812,0.7460895453$ C, $0,-3.4904030968,4.9047485331,-0.1341990517$ $C, 0,-4.3369221628,3.966142144,-0.7644741809$ C, $0,-4.206387407,2.6265656137,-0.493958954$ $C, 0,-3.2236112985,2.1589457167,0.416387423$ C, $0,-3.0427462718,0.766901458,0.7076881124$ $C, 0,0.6728233509,1.6900287167,3.5829322643$ C, $0,-3.4460592504,-1.0339407748,-0.9494002771$ C, $0,-3.9368454263,-0.2434375399,0.0647752618$ C, $0,-5.2862866161,-0.4014711677,0.5256162361$ C, $0,-5.8235535308,0.3745070679,1.5855012674$ C, $0,-7.1206736316,0.2003492532,1.9994903738$ C, $0,-7.943861169,-0.761758905,1.3739673668$ C, $0,-7.4490466549,-1.5332822676,0.3530031134$ C, $0,-6.1111976955,-1.3777221189,-0.0939609757$ C, $0,-5.5857793459,-2.1862072714,-1.1366593313$ $C, 0,-4.2855245508,-2.0275942618,-1.5424516054$ C, $0,-4.450191688,-3.7721835487,-3.1428017412$ $\mathrm{H}, \mathrm{O},-0.7074545751,3.3936511056,2.3867739122$ $\mathrm{H}, \mathrm{O},-1.8646896838,5.1970737698,1.2242798974$ $\mathrm{H}, \mathrm{O},-3.5990302126,5.9624412335,-0.354749192$ $\mathrm{H}, \mathrm{O},-5.0913416664,4.3063308791,-1.4670758066$ $\mathrm{H}, \mathrm{O},-4.8563237956,1.9102538524,-0.9854106086$ $\mathrm{H}, \mathrm{O}, 1.2204559322,2.2603900663,2.8259441035$ $\mathrm{H}, 0,1.3765668909,1.0714301959,4.1390748535$ $\mathrm{H}, 0,0.1680877639,2.3807097559,4.27129332$ $\mathrm{H}, \mathrm{0},-5.1944652904,1.1150798765,2.0677340344$ $\mathrm{H}, \mathrm{O},-7.5161009183,0.8040277265,2.8102932132$ $\mathrm{H}, \mathrm{O},-8.9697254366,-0.8911054671,1.7054523122$ $\mathrm{H}, \mathrm{O},-8.0770474023,-2.2788380446,-0.1274778175$ $\mathrm{H}, \mathrm{O},-6.2321630704,-2.930107481,-1.5882880862$ $\mathrm{H}, \mathrm{O},-4.7861957439,-4.5332374552,-2.4277325199$ $\mathrm{H}, \mathrm{O},-3.7907452543,-4.2375997987,-3.876442525$ $\mathrm{H}, \mathrm{O},-5.321142649,-3.3511349939,-3.6599502612$ $\mathrm{H}, \mathrm{O},-1.934120399,-1.1526850293,-2.4728258652$ $\mathrm{H}, \mathrm{O},-1.6405757718,0.099685527,-1.2577553381$ $\mathrm{H}, 0,-2.7449193406,-1.6412691174,1.8839552672$ 
$\mathrm{H}, 0,-1.3017647544,-1.2187498763,2.801394167$

$\mathrm{H}, 0,0.8936443811,-0.0043873165,-3.0285226654$

$\mathrm{H}, 0,2.5590227894,0.5817036691,-3.1464208431$

$\mathrm{H}, 0,2.0998927899,-0.9340434244,-3.9508551659$

\section{TS- 6' - A}

Opt a wB97XD/SDD/6-31G (d,p) in toluene (SMD model)

$\mathrm{SCF}$ Done: $\mathrm{E}(\mathrm{RwB} 97 \mathrm{XD})=-2877.59427980 \mathrm{a} \cdot \mathrm{u}$.

Zero-point correction $=0.792333$ Hartree/Particle

Sum of electronic and thermal Free Energies = -2876.908356 a.u.

Imaginary Freq. $=-320.5314 \mathrm{~cm}^{-1}$

Sp @ B3LYP-D3 (BJ)/def2-TZVPP in toluene (SMD model)

SCF Done: $\mathrm{E}(\mathrm{RB} 3 \mathrm{LYP})=-2879.51062987 \mathrm{a} \cdot \mathrm{u}$.

$\mathrm{Rh}, 0,0.834751544,-1.6435848464,0.6973987631$

$0,0,0.7672355586,0.3338873885,0.0624833387$

C, $0,1.8607749245,0.9266267319,-0.3157434278$

$\mathrm{C}, 0,3.0663903856,0.8406456709,0.392536873$

C, $0,1.8500516423,1.7119006994,-1.5218382584$

$\mathrm{C}, 0,4.2825792478,1.3368620748,-0.1779639914$

C, $0,2.9822437649,2.2546949,-2.0450689698$

C, $0,4.2358266434,2.0519783626,-1.4083745455$

$\mathrm{H}, 0,2.9406990345,2.8149221942,-2.9738412852$

$\mathrm{C}, 0,6.6521745293,2.2882564169,-1.4185657433$

$\mathrm{C}, 0,6.7135400639,1.5431752655,-0.2217397536$

C, $0,5.5672575604,1.0816367365,0.3792373895$

C, $0,5.4315888363,2.5292616831,-1.9981223531$

$\mathrm{H}, 0,7.5637849377,2.6533505552,-1.8811306698$

$\mathrm{H}, 0,7.6782940362,1.3232734781,0.226372172$

$\mathrm{H}, 0,5.6439554224,0.4937162912,1.2871802391$

$\mathrm{H}, 0,5.3639473425,3.0803580121,-2.9326010036$

$\mathrm{C}, 0,3.033510534,0.2113715012,1.7298788141$

$\mathrm{C}, 0,3.6550837281,0.8607441799,2.8081157726$

$\mathrm{C}, 0,2.3883210385,-1.0251176839,1.9773824011$

C, $0,3.6759567071,0.3155136854,4.0844510435$

$\mathrm{H}, 0,4.1258370165,1.823255071,2.6310693005$

C, $0,2.420689879,-1.5659052588,3.2750435163$

$\mathrm{C}, 0,3.0532911204,-0.9094471675,4.3200585415$

$\mathrm{H}, 0,4.1713929636,0.8458991794,4.8918931448$

$\mathrm{H}, 0,1.9581160547,-2.5305365018,3.4583716708$

$\mathrm{H}, 0,3.0619255729,-1.3512408202,5.312131518$

C, $0,2.4508956269,-1.903049559,-0.5653315947$

$\mathrm{C}, 0,2.9337772925,-2.2359073942,0.5772889754$

C, $0,-0.5940485124,-2.727184987,2.071880619$

$\mathrm{C}, 0,-0.0599711917,-3.6069723797,1.0707140926$

C, $0,-0.5592177432,-3.1706225487,-0.1991163849$

C, $0,-1.2619162193,-1.9633248639,-0.0039738407$

C, $0,-1.3120885352,-1.7000264544,1.4354958023$

C, $0,-1.8760001749,-1.1000057341,-1.0710655137$

$\mathrm{C}, 0,-2.1252293367,-0.646462832,2.1431960841$

$\mathrm{H}, 0,-0.4349505308,-2.8059097551,3.1390695631$

$\mathrm{H}, 0,0.5179166405,-4.5032394638,1.2524145511$

$\mathrm{H}, \mathrm{O},-0.3562127671,-3.6326148896,-1.1559291069$

$\mathrm{Cl}, 0,0.3292485137,1.9218123851,-2.3557330829$

$\mathrm{C}, 0,2.6629490632,-1.6810298077,-1.9723835213$

C, $0,1.5989213591,-1.6547091368,-2.8825051057$

C, $0,3.9641055444,-1.4175224579,-2.4335185035$ 
C, $0,1.8327651809,-1.3969127554,-4.2276323697$

$\mathrm{H}, 0,0.589398216,-1.8113711341,-2.519256688$

C, $0,4.1890864695,-1.156158185,-3.7779548095$

$\mathrm{H}, \mathrm{O}, 4.7848814887,-1.3881596909,-1.7238362019$ $\mathrm{C}, 0,3.1260144996,-1.1474217821,-4.6800073078$ $\mathrm{H}, 0,0.9987009549,-1.3720643993,-4.9221060918$ $\mathrm{H}, 0,5.1969078784,-0.9405568306,-4.1190611538$ $\mathrm{H}, 0,3.3038939612,-0.932494998,-5.7291269873$ C, $0,3.9193395986,-3.1624929697,1.1967133552$ $0,0,-0.3880256616,1.1880682637,3.153317352$ $0,0,-3.712638132,-2.8666639528,-2.0459529322$ $C, 0,-2.0401769769,0.7428025443,1.5535322578$ C, $0,-1.1014350551,1.6655689478,2.1072391397$ C, $0,-0.9817179381,2.9351732542,1.6042626044$ $\mathrm{C}, 0,-1.7692365842,3.3422170736,0.4974873443$ $\mathrm{C}, 0,-1.6277750375,4.6354663511,-0.0688580414$ $C, 0,-2.3732654929,5.0132797975,-1.1564360534$ C, $0,-3.2998574357,4.1137070582,-1.72813976$ C, $0,-3.4628897337,2.8587032952,-1.1963687244$ $\mathrm{C}, 0,-2.7072873377,2.4407067878,-0.0705667021$ $\mathrm{C}, 0,-2.8347068104,1.132279691,0.4997806904$ $\mathrm{C}, 0,0.5586668444,2.0472324359,3.7508260627$ $\mathrm{C}, 0,-3.3533370878,-0.8946064752,-0.8308653249$ C, $0,-3.8137893,0.1623428479,-0.0789205404$ $\mathrm{C}, 0,-5.2153548919,0.3163370223,0.1840252529$ $\mathrm{C}, 0,-5.7282777192,1.3741188472,0.9791992275$ $\mathrm{C}, 0,-7.0761466951,1.4928753524,1.2101885224$ $\mathrm{C}, 0,-7.9775673835,0.555652569,0.6590982195$ C, $0,-7.5100148677,-0.4831150916,-0.1056388268$ $\mathrm{C}, 0,-6.1218289512,-0.6326942915,-0.3594062343$ C, $0,-5.6282746556,-1.7175713499,-1.1316355547$ $C, 0,-4.2806702411,-1.8515223605,-1.3481896047$ $\mathrm{C}, 0,-4.5626927999,-3.8577381611,-2.5833179848$ $\mathrm{H}, 0,-0.2702476771,3.6405607174,2.0169365101$ $\mathrm{H}, \mathrm{O},-0.9052324275,5.3194672163,0.3680744498$ $\mathrm{H}, \mathrm{O},-2.2484580263,6.0031517493,-1.5850146209$ $\mathrm{H}, \mathrm{O},-3.8826400428,4.415485391,-2.5928706631$ $\mathrm{H}, 0,-4.1729991353,2.1701972288,-1.6422299973$ $\mathrm{H}, 0,1.3109949663,2.3779823061,3.0274215544$ $\mathrm{H}, 0,1.0480887456,1.4633893806,4.5299844494$ $\mathrm{H}, 0,0.0715000282,2.9221147128,4.2015573836$ $\mathrm{H}, 0,-5.038829416,2.0955415452,1.4043731642$ $\mathrm{H}, 0,-7.4516975295,2.3091257122,1.8192197172$ $\mathrm{H}, \mathrm{O},-9.0423878345,0.6575441774,0.8457111974$ $\mathrm{H}, 0,-8.1992408514,-1.2104651575,-0.526420433$ $\mathrm{H}, \mathrm{O},-6.3385136787,-2.4341072865,-1.528289245$ $\mathrm{H}, \mathrm{O},-5.1198507243,-4.3795516244,-1.7954258672$ $\mathrm{H}, 0,-3.9138313795,-4.5713200779,-3.0926453093$ $\mathrm{H}, 0,-5.2686730884,-3.4345449262,-3.3084483581$ $\mathrm{H}, \mathrm{O},-1.7158956662,-1.571515918,-2.042235148$ $\mathrm{H}, \mathrm{O},-1.3536687696,-0.1382625179,-1.0733376003$ $\mathrm{H}, 0,-3.1697442131,-0.9775542056,2.1294176422$ $\mathrm{H}, 0,-1.8080240541,-0.6186875058,3.1865956945$ $\mathrm{H}, \mathrm{O}, 3.4261318474,-3.8669085456,1.8725618848$ $\mathrm{H}, 0,4.4152201044,-3.7322643544,0.4056115602$ $\mathrm{H}, 0,4.6703274856,-2.6154379917,1.7738102638$ 


\section{TS-6'-B}

Opt a wB97XD/SDD/6-31G(d,p) in toluene (SMD model)

$\mathrm{SCF}$ Done: $\mathrm{E}(\mathrm{RwB} 97 \mathrm{XD})=-2877.58766520 \mathrm{a} \cdot \mathrm{u}$.

Zero-point correction $=0.791935$ Hartree/Particle

Sum of electronic and thermal Free Energies = -2876.904574 a.u.

Imaginary Freq. $=-316.6243 \mathrm{~cm}^{-1}$

$\mathrm{Sp}$ @ B3LYP-D3 (BJ)/def2-TZVPP in toluene (SMD model)

$\mathrm{SCF}$ Done: $\mathrm{E}(\mathrm{RB} 3 \mathrm{LYP})=-2879.50368308 \mathrm{a} \cdot \mathrm{u}$.

$\mathrm{Rh}, 0,-0.7561505713,-1.3738500301,-0.3467642055$

$0,0,-0.352505901,0.6336307063,-0.0064930685$

$\mathrm{C}, 0,-1.2888842676,1.495961667,0.2516641013$

C, $0,-2.5305784325,1.5273552735,-0.3997358386$

C, $0,-1.0389761217,2.4999993933,1.2537057837$

$\mathrm{C}, 0,-3.5630407573,2.4187053779,0.0442148736$

C, $0,-1.9742025822,3.4168647389,1.6232181095$

$\mathrm{C}, 0,-3.2724144917,3.385291649,1.0497400329$

$\mathrm{H}, \mathrm{O},-1.7444786084,4.1546981972,2.3858360713$

C, $0,-5.5537457323,4.2143643286,0.9899200394$

$C, 0,-5.8671573133,3.2276006721,0.0310241362$

C, $0,-4.9059301058,2.3594706031,-0.4259543586$

C, $0,-4.2786864611,4.2795379009,1.4917602334$

$\mathrm{H}, 0,-6.3195597298,4.9000514472,1.3384163568$

$\mathrm{H}, \mathrm{O},-6.8836350425,3.1448603344,-0.3427043184$

$\mathrm{H}, \mathrm{O},-5.1832216375,1.5973336586,-1.1447435817$

$\mathrm{H}, \mathrm{O},-4.0218146491,5.0138502849,2.2510246625$

C, $0,-2.7191157605,0.6435730227,-1.5711524027$

$\mathrm{C}, 0,-3.2945250787,1.1719256952,-2.7410084358$

$C, 0,-2.3213685926,-0.7125055669,-1.5876653328$

C, $0,-3.5076084243,0.3996969673,-3.8736309862$

$\mathrm{H}, \mathrm{O},-3.5666849872,2.2229856494,-2.7558108279$

C, $0,-2.5618797868,-1.4890166721,-2.7345344058$

$\mathrm{C}, 0,-3.1393247056,-0.945600846,-3.8709893943$

$\mathrm{H}, 0,-3.9568601622,0.8466705937,-4.7552685128$

$\mathrm{H}, \mathrm{O},-2.3120773478,-2.5448942121,-2.7169436371$

$\mathrm{H}, \mathrm{O},-3.3097769978,-1.5673278725,-4.7447563869$

C, $0,-2.1824946167,-1.2158020483,1.1079230742$

$\mathrm{C}, 0,-2.9181283565,-1.530702897,0.1082060341$

C, $0,0.3901118412,-2.8290327989,-1.6822488539$

C, $0,-0.2157887598,-3.4832657568,-0.5545769919$

$C, 0,0.4341918369,-2.9957075773,0.6250045822$

$\mathrm{C}, 0,1.3086302305,-1.9535622878,0.248196644$

C, $0,1.3085979293,-1.8780509123,-1.2144386337$

C, $0,2.1237296993,-1.0876948383,1.1690424754$

C, $0,2.2310465955,-1.0624709563,-2.0831953765$

$\mathrm{H}, \mathrm{O}, \mathrm{O} .1399312699,-2.9942586385,-2.7218244043$

$\mathrm{H}, \mathrm{O},-0.9429376553,-4.2830746997,-0.5996819968$

$\mathrm{H}, \mathrm{0}, 0.2304490416,-3.3038703671,1.6417891701$

$\mathrm{Cl}, 0,0.5225806537,2.5026045947,2.0396218701$

C, $0,-2.0537825802,-0.9516471976,2.5508051668$

C, $0,-4.1391559875,-2.2942596823,-0.1797165684$

$\mathrm{C}, 0,-4.3209891233,-3.517363935,0.4783166172$

C, $0,-5.1467045941,-1.8203314071,-1.0268503714$

$\mathrm{C}, 0,-5.4857242453,-4.2543667258,0.2894097574$

$\mathrm{H}, \mathrm{O},-3.5398850533,-3.8839825224,1.1377448681$

$\mathrm{C}, 0,-6.3112144874,-2.5561960635,-1.2086143951$

$\mathrm{H}, \mathrm{O},-5.0157920263,-0.8727072627,-1.53747239$

$\mathrm{C}, 0,-6.482580867,-3.7757492893,-0.5561751417$ 
$\mathrm{H}, \mathrm{O},-5.6131015868,-5.2025508646,0.8025743057$

$\mathrm{H}, \mathrm{0},-7.0893139407,-2.1750413003,-1.8625356751$

$\mathrm{H}, \mathrm{O},-7.3920522614,-4.3494757896,-0.7058066511$

$0,0,0.7363871143,0.9085326444,-3.2147424753$

$0,0,3.7068618564,-3.0168902771,2.2784712889$

C, $0,2.4123499379,0.3795459032,-1.6666082613$

C, $0,1.5976593679,1.3776546692,-2.2826651788$

C, $0,1.7269310324,2.6999451173,-1.944497743$

C, $0,2.652105406,3.0940135241,-0.9446555896$

C, $0,2.7751407146,4.4516260467,-0.5510393113$

C, $0,3.6503855248,4.8219769218,0.4380393535$

$C, 0,4.4445466177,3.8484491371,1.0832282515$

C, $0,4.352088767,2.5285770766,0.7169161176$

C, $0,3.4621759792,2.1171215513,-0.3086959123$

$C, 0,3.330918851,0.7477884111,-0.7104839162$

$C, 0,-0.1346074856,1.8319107893,-3.83068908$

$C, 0,3.5959975565,-1.1692286869,0.8395925252$

C, $0,4.1737188135,-0.3015316342,-0.0596766144$

C, $0,5.5609413342,-0.4222416028,-0.4043261948$

C, $0,6.1881865179,0.4342987203,-1.3463009727$

C, 0, 7.518502095, 0.2915815123,-1.6531082649

C, $0,8.2867894592,-0.7185805514,-1.0335607405$

$C, 0,7.7054464102,-1.5676390398,-0.1259961318$

C, $0,6.3316846274,-1.4458851771,0.2086556729$

C, $0,5.7177503846,-2.3336981936,1.1316113586$

C, $0,4.3850123384,-2.2053627754,1.4289610361$

C, $0,4.4210153268,-4.057653273,2.911115982$

$\mathrm{H}, 0,1.1132649274,3.4632543228,-2.4084269638$

$\mathrm{H}, 0,2.1509900231,5.1943112058,-1.0408329844$

$\mathrm{H}, 0,3.728511394,5.864088601,0.7331070462$

$\mathrm{H}, 0,5.1280495857,4.1458643431,1.8724554053$

$\mathrm{H}, 0,4.9612111139,1.7839486213,1.2185153197$

$\mathrm{H}, 0,-0.7566695886,2.3460546368,-3.0903707203$

$\mathrm{H}, 0,-0.7759800089,1.2483094725,-4.4907711055$

$\mathrm{H}, 0,0.4215256103,2.5716197934,-4.421584307$

$\mathrm{H}, 0,5.6012547241,1.2109411118,-1.8246690218$

$\mathrm{H}, 0,7.9823424793,0.9568626877,-2.3745757531$

$\mathrm{H}, 0,9.3391064813,-0.8231497947,-1.2799489356$

$\mathrm{H}, 0,8.2916583161,-2.3498065492,0.3490412354$

$\mathrm{H}, 0,6.3245216407,-3.1122297218,1.5797761594$

$\mathrm{H}, \mathrm{O}, 4.8283850116,-4.7681814746,2.1812703933$

$\mathrm{H}, \mathrm{O}, 3.7035054713,-4.5744855528,3.5496550177$

$\mathrm{H}, 0,5.2372476927,-3.6672795062,3.5313487901$

$\mathrm{H}, 0,1.9456403662,-1.4033015036,2.1984951947$

$\mathrm{H}, 0,1.7705259813,-0.056801895,1.0688366934$

$\mathrm{H}, 0,3.207267908,-1.5603265236,-2.0762652056$

$\mathrm{H}, 0,1.8554376784,-1.1012060071,-3.1066062086$

$\mathrm{H}, 0,-1.511352073,-0.0172995896,2.7251711607$

$\mathrm{H}, \mathrm{O},-3.0408358221,-0.8778610608,3.0183749992$

$\mathrm{H}, \mathrm{O},-1.489239278,-1.7533690079,3.0383964489$

\section{INT-7 ' - A}

Opt a wB97XD/SDD/6-31G(d,p) in toluene (SMD model)

SCF Done: E (RwB97XD) $=-2877.65255390 \mathrm{a} \cdot \mathrm{u}$.

Zero-point correction $=0.794844$ Hartree/Particle

Sum of electronic and thermal Free Energies = -2876.965294 a.u. @ $358.15 \mathrm{~K}$

Sp \& B3LYP-D3 (BJ)/def2-TZVPP in toluene (SMD model) 
SCF Done: E (RB3LYP) $=-2879.55948581$ a.u.

$\mathrm{Rh}, 0,0.6576475471,1.0415548007,-0.9561568994$

$0,0,0.5663578968,-0.4289380346,0.4890919919$

C, $0,1.5634060887,-1.2627523822,0.5331778936$

C, $0,2.5557069262,-1.3179686212,-0.4452028078$

$C, 0,1.6569209292,-2.1886716023,1.6297250535$

$\mathrm{C}, 0,3.6025026169,-2.286166505,-0.385357252$

$\mathrm{C}, 0,2.6285471494,-3.1419340303,1.6952288846$

C, $0,3.6259804188,-3.2244290923,0.6876311052$

$\mathrm{H}, 0,2.6587354859,-3.8315117158,2.5333932386$

$\mathrm{C}, 0,5.627430841,-4.2657908413,-0.2096055174$

$\mathrm{C}, 0,5.6235672051,-3.3274157804,-1.2650130119$

C, $0,4.6466444732,-2.3664370128,-1.3507042944$

C, $0,4.6468722508,-4.2052673785,0.7483651933$

$\mathrm{H}, 0,6.405532738,-5.02055438,-0.1550184397$

$\mathrm{H}, 0,6.4063298088,-3.3623715191,-2.0172921512$

$\mathrm{H}, 0,4.6737734768,-1.6538426777,-2.1683561786$

$\mathrm{H}, 0,4.6410208098,-4.9102416311,1.5758209952$

C, $0,2.5160997504,-0.3664081987,-1.6050923369$

C, $0,2.2945107625,-0.9056938437,-2.9067120174$

C, $0,3.0158535879,0.9642000184,-1.5185817071$

$\mathrm{C}, 0,2.5962825211,-0.2025615887,-4.0483025521$

$\mathrm{H}, 0,1.9144648692,-1.9180643331,-2.9742434243$

$\mathrm{C}, 0,3.3354397484,1.6618576426,-2.7143030666$

C, $0,3.1450421639,1.0909222016,-3.950404093$

$\mathrm{H}, 0,2.431945311,-0.6514836852,-5.0229434637$

$\mathrm{H}, 0,3.74362621,2.6648582837,-2.6285820738$

$\mathrm{H}, 0,3.4121134181,1.636851467,-4.8501417814$

C, $0,1.9953992067,1.8790949365,0.3267001018$

C, $0,3.20258879,1.6360941743,-0.1888740795$

$\mathrm{Cl}, 0,0.4850599833,-2.0553743365,2.9176215953$

$\mathrm{C}, 0,1.6244117062,2.6016347428,1.5512847565$

C, $0,0.726338089,2.0369648844,2.4693413073$

C, $0,2.1245022245,3.8870441248,1.8025963976$

C, $0,0.3539555966,2.7355594939,3.6119449799$

$\mathrm{H}, 0,0.3394729635,1.0425178142,2.2691992255$

C, $0,1.7458634619,4.5852939833,2.9463703551$

$\mathrm{H}, 0,2.8075473504,4.3396623586,1.0889645714$

C, $0,0.8598285363,4.0121312109,3.8547666303$

$\mathrm{H}, \mathrm{O},-0.3354124847,2.2801513685,4.3169622427$

$\mathrm{H}, 0,2.1429425962,5.5802721464,3.1260564548$

$\mathrm{H}, 0,0.5641099087,4.5567180705,4.7465084942$

C, $0,4.5725184827,1.9274465344,0.3481815897$

$0,0,-0.475615559,-2.7334556155,-1.7344602973$

$0,0,-3.8803762726,3.5235555227,0.1906623151$

$\mathrm{C}, 0,-0.6521795991,2.7710116802,-1.1033568121$

C, $0,-0.03331028,2.5555701158,-2.3604852941$

$\mathrm{C}, 0,-0.5233784083,1.2904107386,-2.8695050759$

$\mathrm{C}, 0,-1.385597186,0.707270801,-1.936123387$

C, $0,-1.4093429989,1.5997258044,-0.7766219483$

C, $0,-2.1503597406,-0.5754382564,-2.1434846921$

$C, 0,-2.2967953002,-1.4716214905,-0.9315423206$

$\mathrm{C}, 0,-1.4093663176,-2.5802395078,-0.7620880188$

$\mathrm{C}, 0,-1.5396368146,-3.4255817696,0.3096213935$

C, $0,-2.5486475984,-3.2077168206,1.2805405031$

$C, 0,-2.685947248,-4.0653383657,2.4028031196$

$C, 0,-3.6562324777,-3.8401371171,3.3455016608$ 
C, $0,-4.5334089947,-2.740839194,3.2113141899$

$\mathrm{C}, 0,-4.4212493898,-1.8940412845,2.1372979674$

C, $0,-3.432795062,-2.1075112687,1.1408205774$

$\mathrm{C}, 0,-3.2844431479,-1.2495190919,0.0030508984$ $\mathrm{C}, 0,0.3763622545,-3.858969082,-1.6473313483$ C, $0,-2.2144871926,1.385146871,0.4746455051$ C, $0,-3.6693049319,1.1921775126,0.1129339093$

$C, 0,-4.1799975391,-0.0611637601,-0.1397247222$ $C, 0,-5.5440836965,-0.2249210406,-0.5500398243$ C, $0,-6.1043245974,-1.4966567357,-0.8381997157$ $\mathrm{C}, 0,-7.4153022857,-1.6177569345,-1.2265076608$ $\mathrm{C}, 0,-8.2288469793,-0.4698674845,-1.3502926754$ C, $0,-7.7120056023,0.7735763665,-1.0870177346$ $C, 0,-6.3598014178,0.9301945466,-0.6865813461$ $\mathrm{C}, 0,-5.813531594,2.2154748647,-0.4276105972$ C, $0,-4.5008590576,2.342912339,-0.0513750655$ $\mathrm{C}, 0,-4.6377946565,4.7093747315,0.0704833558$ $\mathrm{H}, \mathrm{0},-0.5153136439,3.6209366406,-0.4485774536$ $\mathrm{H}, \mathrm{O}, 0.6252550404,3.2359908068,-2.881392802$ $\mathrm{H}, 0,-0.2158800591,0.8361366094,-3.8030309597$ $\mathrm{H}, \mathrm{O},-1.660223678,-1.1307833876,-2.9453508341$ $\mathrm{H}, 0,-3.1517950744,-0.3094875167,-2.5012393095$ $\mathrm{H}, \mathrm{O},-0.8640909174,-4.2598555471,0.4541019081$ $\mathrm{H}, \mathrm{O},-1.9998286745,-4.9015516097,2.5063522557$ $\mathrm{H}, \mathrm{O},-3.7489875789,-4.5025766082,4.2009008517$ $\mathrm{H}, 0,-5.2950102341,-2.562783508,3.9642081482$ $\mathrm{H}, 0,-5.093889461,-1.0480687141,2.0444045115$ $\mathrm{H}, 0,1.0082926068,-3.8191283458,-0.7534109282$ $\mathrm{H}, \mathrm{O}, 1.0110944711,-3.8370425331,-2.5338464118$ $\mathrm{H}, 0,-0.1983724572,-4.7936155951,-1.6518531693$ $\mathrm{H}, 0,-2.0868791692,2.2452609346,1.1330003696$ $\mathrm{H}, 0,-1.8324951203,0.5026535869,0.9938993374$ $\mathrm{H}, \mathrm{O},-5.4819504314,-2.3801465976,-0.7429890457$ $\mathrm{H}, \mathrm{O},-7.8294595832,-2.5981927304,-1.4396798884$ $\mathrm{H}, 0,-9.2656938914,-0.574312067,-1.6553230655$ $\mathrm{H}, 0,-8.3339570317,1.659584918,-1.1837737523$ $\mathrm{H}, \mathrm{0},-6.4537336562,3.0821145444,-0.5471492796$ $\mathrm{H}, 0,-5.0150611691,4.8437350666,-0.9508674381$ $\mathrm{H}, \mathrm{O},-3.9595006479,5.5286270669,0.3116343732$ $\mathrm{H}, 0,-5.4797608691,4.7211159425,0.7732979072$ $\mathrm{H}, 0,5.1176335617,2.6317884568,-0.2926526903$ $\mathrm{H}, 0,4.517404192,2.3527312089,1.3532957635$ $\mathrm{H}, 0,5.1669594219,1.007589966,0.3967029525$

\section{INT- $7^{\prime}-\mathrm{B}$}

Opt a wB97XD/SDD/6-31G(d,p) in toluene (SMD model) $\mathrm{SCF}$ Done: $\mathrm{E}(\mathrm{RwB} 97 \mathrm{XD})=-2877.64651958 \mathrm{a} \cdot \mathrm{u}$. Zero-point correction $=0.794596$ Hartree/Particle Sum of electronic and thermal Free Energies = -2876.959816 a.u. $\mathrm{Sp}$ a B3LYP-D3 (BJ)/def2-TZVPP in toluene (SMD model) $\mathrm{SCF}$ Done: $\mathrm{E}(\mathrm{RB} 3 \mathrm{LYP})=-2879.55477846 \mathrm{a} \cdot \mathrm{u}$.

$\mathrm{Rh}, 0,0.6858301539,1.0869733132,-1.047003192$ $0,0,0.5974843984,-0.3624379123,0.4131056215$ $\mathrm{C}, 0,1.5761116467,-1.2168377531,0.4775047659$ C, $0,2.5641152951,-1.3332384793,-0.5013488697$ C, $0,1.6539587195,-2.0994522497,1.6099861067$ 
C, $0,3.5855999908,-2.3262394428,-0.4077280283$

C, $0,2.594252186,-3.0807465849,1.7038013787$

C, $0,3.5814575383,-3.2303507824,0.6942253576$

$\mathrm{H}, 0,2.609717453,-3.7384402587,2.5676225286$

C, $0,5.5540159885,-4.3552538257,-0.163888577$

$C, 0,5.5830581818,-3.4456135705,-1.2437187396$

C, $0,4.6339404252,-2.4607303591,-1.3618643337$

C, $0,4.5728301567,-4.2384968041,0.7876612497$

$\mathrm{H}, 0,6.3102033696,-5.1296202213,-0.0830571287$

$\mathrm{H}, \mathrm{O}, 6.3716607708,-3.5205903088,-1.9868115178$

$\mathrm{H}, 0,4.6892503007,-1.7661928265,-2.1931814554$

$\mathrm{H}, 0,4.543655932,-4.9172492174,1.6363603795$

C, $0,2.5658003761,-0.3909153972,-1.6657171564$

C, $0,2.3962799893,-0.9146878192,-2.978839192$

C, $0,3.0226886381,0.9489785446,-1.5368133533$

C, $0,2.6963004452,-0.1737889619,-4.0977328627$

$\mathrm{H}, 0,2.061880088,-1.9408355871,-3.0770651779$

C, $0,3.3105643176,1.6968478831,-2.7118153513$

C, $0,3.170545773,1.1451807578,-3.9637615513$

$\mathrm{H}, 0,2.5766984036,-0.6096623422,-5.0849091227$

$\mathrm{H}, 0,3.6530482195,2.7209539932,-2.5954269077$

$\mathrm{H}, 0,3.4180645332,1.7267902131,-4.8464156647$

$\mathrm{C}, 0,1.9857382592,1.873436068,0.292764938$

C, $0,3.1995087219,1.5510116632,-0.1685261644$

$\mathrm{Cl}, 0,0.508394674,-1.8692039504,2.908180356$

$C, 0,1.5588176625,2.5047876527,1.5735833096$

C, $0,4.5250372286,1.6258116862,0.4846471017$

$C, 0,4.6566587666,1.3564434109,1.8540272853$

C, $0,5.6833373659,1.9136984029,-0.2488859539$

C, $0,5.899269922,1.4159584203,2.4757416059$

$\mathrm{H}, 0,3.7811246753,1.0655696813,2.4255303925$

$\mathrm{C}, 0,6.9268425061,1.966121109,0.371775655$

$\mathrm{H}, 0,5.6124082249,2.0968781453,-1.316909596$

C, 0, 7.0394491154,1.7261669812,1.7388690212

$\mathrm{H}, 0,5.9787792529,1.2006320414,3.5371474405$

$\mathrm{H}, 0,7.8114240365,2.194280662,-0.2155746861$

$\mathrm{H}, 0,8.0101803671,1.7660915624,2.223523653$

$0,0,-0.4560489037,-2.7034448158,-1.7809827091$

$0,0,-3.8554753384,3.5701685974,0.1539169135$

C, $0,-0.6319996783,2.8116843526,-1.2404804287$

$C, 0,-0.0255780849,2.5589682319,-2.4955757284$

C, $0,-0.5192876929,1.2795777044,-2.9636517418$

C, $0,-1.3684913902,0.7238443596,-2.0042388536$

C, $0,-1.3766357996,1.6462184901,-0.8662860602$

C, $0,-2.1423392828,-0.5594940932,-2.1673975941$

C, $0,-2.2520582767,-1.4358292319,-0.9380183832$

C, $0,-1.3573964855,-2.5399239789,-0.780794745$

C, $0,-1.4511506306,-3.3707782425,0.3057877278$

C, $0,-2.4292171816,-3.1393046958,1.3052044878$

C, $0,-2.5256417074,-3.978180822,2.4454993951$

C, $0,-3.4625532603,-3.738759603,3.4179116051$

C, $0,-4.3468916363,-2.6438384152,3.2955046458$

$C, 0,-4.2748066411,-1.8155185232,2.2036075739$

$C, 0,-3.321074544,-2.0438167449,1.1770092606$

C, $0,-3.2120048204,-1.2024248724,0.0219257992$

C, $0,0.3868892835,-3.8368445545,-1.7175632868$

C, $0,-2.1526478412,1.4568597855,0.4084857529$

C, $0,-3.6157205324,1.2398162044,0.0971786993$ 
$C, 0,-4.1206935527,-0.0233922016,-0.1154296411$ C, $0,-5.4949246946,-0.2077388805,-0.4813967317$ C, $0,-6.0460954338,-1.4891332338,-0.7427882273$ $\mathrm{C}, 0,-7.3669530398,-1.6309685723,-1.0884447608$ C, $0,-8.2011732737,-0.4958378117,-1.1905963729$ $\mathrm{C}, 0,-7.6938535965,0.7565486864,-0.9528541035$ $\mathrm{C}, 0,-6.3312265781,0.9346300911,-0.5986933126$ $\mathrm{C}, 0,-5.7925880601,2.2294090273,-0.373982223$ $\mathrm{C}, 0,-4.4681256932,2.3774921307,-0.0504617799$ $\mathrm{C}, 0,-4.6364031122,4.7423156204,0.0499882676$ $\mathrm{H}, \mathrm{O},-0.4970595263,3.6885208405,-0.6222786532$ $\mathrm{H}, 0,0.6249624429,3.2251626492,-3.0442118506$ $\mathrm{H}, \mathrm{O},-0.2253871637,0.8014782351,-3.8894866865$ $\mathrm{H}, \mathrm{O},-1.6799258588,-1.1291207915,-2.9755314984$ $\mathrm{H}, \mathrm{O},-3.1535662152,-0.2939237658,-2.4970269023$ $\mathrm{H}, \mathrm{0},-0.7692200335,-4.2016265548,0.4402760778$ $\mathrm{H}, \mathrm{O},-1.8328891998,-4.8100724673,2.539279639$ $\mathrm{H}, \mathrm{O},-3.5230393899,-4.3868777545,4.2869974495$ $\mathrm{H}, \mathrm{O},-5.0821540804,-2.4544354858,4.0713239693$ $\mathrm{H}, 0,-4.9511466841,-0.9716225921,2.1199744208$ $\mathrm{H}, 0,1.0516825987,-3.7995107978,-0.8478598551$ $\mathrm{H}, 0,0.988731381,-3.8266633322,-2.6268294695$ $\mathrm{H}, \mathrm{O},-0.1983488553,-4.7647983197,-1.6950794698$ $\mathrm{H}, 0,-2.0213739159,2.337639062,1.0389551062$ $\mathrm{H}, \mathrm{0},-1.7450768111,0.5946153795,0.9427527673$ $\mathrm{H}, \mathrm{O},-5.4079983557,-2.3629564727,-0.6643461046$ $\mathrm{H}, \mathrm{O},-7.773303386,-2.618374662,-1.2837990172$ $\mathrm{H}, 0,-9.2456980357,-0.6171199335,-1.4611433744$ $\mathrm{H}, \mathrm{O},-8.3305179879,1.6333426692,-1.0363376957$ $\mathrm{H}, 0,-6.4480308527,3.0866941551,-0.4776836238$ $\mathrm{H}, \mathrm{O},-5.0531281122,4.8604165942,-0.9578602928$ $\mathrm{H}, 0,-3.9636339437,5.5754670947,0.256658091$ $\mathrm{H}, 0,-5.4518317474,4.7491950924,0.7837071325$ $\mathrm{H}, 0,2.4011434979,2.9326644653,2.128320035$ $\mathrm{H}, 0,0.8317472921,3.3030651326,1.3913610258$ $\mathrm{H}, 0,1.0649457724,1.7571907739,2.2047354404$

\section{TS-7'-A}

Opt @ wB97XD/SDD/6-31G(d,p) in toluene (SMD model) $\mathrm{SCF}$ Done: $\mathrm{E}(\mathrm{RwB} 97 \mathrm{XD})=-2877.62483669 \mathrm{a} \cdot \mathrm{u}$. Zero-point correction $=0.793514$ Hartree/Particle Sum of electronic and thermal Free Energies = -2876.936051 a.u. @ $358.15 \mathrm{~K}$ Imaginary Freq. $=-78.4808 \mathrm{~cm}^{-1}$

$\mathrm{Sp}$ a B3LYP-D3 (BJ)/def2-TZVPP in toluene (SMD model) $\mathrm{SCF}$ Done: $\mathrm{E}(\mathrm{RB} 3 \mathrm{LYP})=-2879.53928637 \mathrm{a} \cdot \mathrm{u}$.

$\mathrm{Rh}, 0,-0.6805289277,-0.9029831583,-0.4711153282$

$0,0,-0.3979116123,1.2211224407,-0.2109516998$

C, $0,-1.3148340875,1.6957359858,0.557937696$

$\mathrm{C}, 0,-2.6664143503,1.8095145274,0.1477768604$ C, $0,-1.0226964989,2.0730692727,1.9243402063$ $\mathrm{C}, 0,-3.7021953116,2.1724908795,1.0683342006$ $\mathrm{C}, 0,-2.0014847825,2.362054386,2.8216991992$ C, $0,-3.3742620617,2.3896664653,2.4314956359$ $\mathrm{H}, 0,-1.7443149713,2.5714109772,3.8557782831$ $\mathrm{C}, 0,-5.7162325726,2.6738296164,2.9828492773$ C, $0,-6.0475107098,2.4753395716,1.6296559063$ 
$C, 0,-5.066339616,2.2292535831,0.6939908873$

C, $0,-4.3964227428,2.6339294991,3.3727572735$

$\mathrm{H}, \mathrm{O},-6.4975814419,2.8608198473,3.7126293076$

$\mathrm{H}, \mathrm{O},-7.0878603438,2.5104195558,1.319969287$

$\mathrm{H}, \mathrm{O},-5.3357719062,2.0669403048,-0.3444727864$

$\mathrm{H}, \mathrm{O},-4.1247355326,2.788474303,4.4135489853$

C, $0,-2.9442229394,1.7074412197,-1.3063499249$

C, $0,-3.2176270288,2.8479197162,-2.0628708702$

C, $0,-2.9279483958,0.4488516238,-1.9295697922$

C, $0,-3.4769658218,2.7550998148,-3.4273576203$

$\mathrm{H}, \mathrm{O},-3.2329128703,3.8151625368,-1.5685420085$

$C, 0,-3.1831164116,0.3638456309,-3.3027668425$

$C, 0,-3.4577478815,1.5070454052,-4.0453227273$

$\mathrm{H}, \mathrm{O},-3.6951357784,3.6498347439,-4.0018966284$

$\mathrm{H}, \mathrm{O},-3.1718247763,-0.6031717946,-3.7953934514$

$\mathrm{H}, \mathrm{O},-3.6593605142,1.4213851378,-5.1089456096$

C, $0,-2.4298708234,-0.6549443991,0.2908359838$

C, $0,-2.7180407244,-0.7585236204,-1.0959890847$

C, $0,-3.4328237985,-2.0239763014,-1.555003898$

C, $0,-3.1132202585,-0.9196647191,1.5242969171$

C, $0,-4.5206160613,-0.9384092574,1.568279868$

C, $0,-2.386736387,-1.0368170419,2.7217928829$

C, $0,-5.1788977673,-1.0844148348,2.7787915033$

$\mathrm{H}, \mathrm{O},-5.0806905575,-0.8197814087,0.6466238175$

C, $0,-3.0509740976,-1.1853099525,3.9312696176$

$\mathrm{H}, 0,-1.3030718993,-0.990582702,2.6775138285$

C, $0,-4.44462878,-1.2047703646,3.9597313428$

$\mathrm{H}, \mathrm{O},-6.2634004865,-1.0876206036,2.8094236181$

$\mathrm{H}, \mathrm{O},-2.4857871164,-1.270952529,4.8536868623$

$\mathrm{H}, \mathrm{O},-4.963374509,-1.3083249787,4.9079162551$

$\mathrm{Cl}, 0,0.6440588272,2.0655458834,2.4381591507$

$0,0,1.0574772833,1.1602683782,-3.0160488343$

$0,0,3.6589931497,-3.0633638807,2.3581051934$

$C, 0,0.360537353,-2.5828664719,0.6836354355$

$C, 0,-0.3208249413,-3.0208881888,-0.506819253$

C, $0,0.4113818566,-2.5153813188,-1.6407789394$

C, $0,1.4126875778,-1.6598568637,-1.1580188388$

C, $0,1.3819360101,-1.7006936789,0.3029341953$

C, $0,2.4370212533,-0.9543457321,-2.0108022872$

$C, 0,2.7538776452,0.4549618367,-1.5661571833$

C, $0,1.9892638962,1.5306765209,-2.1095574326$

C, $0,2.2185616553,2.8245660637,-1.7180757909$

C, $0,3.2036922949,3.1116044799,-0.7404632561$

C, $0,3.4337559067,4.4398410446,-0.2953386007$

C, $0,4.3685576235,4.7069938659,0.6720877123$

C, $0,5.1186372136,3.655184644,1.2440192999$

C, $0,4.9193938703,2.361121585,0.8316408251$

C, $0,3.9673929381,2.0552774225,-0.1756461268$

$C, 0,3.7196668621,0.7158214887,-0.6205079967$

C, $0,0.0755450609,2.1130031566,-3.3740463555$

C, $0,2.3094008517,-0.9629139458,1.2321513409$

C, $0,3.7641675151,-1.2216255751,0.911193947$

C, $0,4.4444245236,-0.4260726384,0.0158073013$

C, $0,5.8082864821,-0.7052631419,-0.3242706438$

C, $0,6.5297731291,0.0675187738,-1.2708371502$

C, $0,7.8359236974,-0.2281603078,-1.5728685101$

C, $0,8.4828508976,-1.3147588612,-0.9435144271$

C, $0,7.8070948,-2.0850488667,-0.0310066402$ 
$C, 0,6.4552440328,-1.8060685042,0.2981066877$

$\mathrm{C}, 0,5.7417581687,-2.6118790916,1.2245247086$

C, $0,4.428381557,-2.3362735591,1.5103650734$

$\mathrm{C}, 0,4.245215293,-4.1802939447,2.9912080858$

$\mathrm{H}, 0,0.0982575803,-2.8434222123,1.7003482591$

$\mathrm{H}, \mathrm{0},-1.1204261661,-3.748562728,-0.5458073838$

$\mathrm{H}, \mathrm{0}, \mathrm{0} .1938848049,-2.7084203177,-2.6828676286$

$\mathrm{H}, 0,2.0775022847,-0.9422712658,-3.0409432572$

$\mathrm{H}, \mathrm{0}, 3.3556088264,-1.5505319376,-1.9925647068$

$\mathrm{H}, 0,1.6319097657,3.6433722072,-2.1176991693$

$\mathrm{H}, \mathrm{0}, 2.8455032456,5.2436568873,-0.7300336324$

$\mathrm{H}, 0,4.5304860194,5.7276818021,1.0055965642$

$\mathrm{H}, 0,5.8509485546,3.871714576,2.0156678211$

$\mathrm{H}, 0,5.4935331144,1.5555207913,1.2774549191$

$\mathrm{H}, \mathrm{O},-0.4272900558,2.4960850149,-2.4818303444$

$\mathrm{H}, \mathrm{O},-0.644641733,1.5835533118,-3.9967597634$

$\mathrm{H}, 0,0.5096234827,2.9390320445,-3.9526823858$

$\mathrm{H}, 0,2.089456134,-1.2678658468,2.2569733832$

$\mathrm{H}, 0,2.1075954093,0.1071883296,1.1583747278$

$\mathrm{H}, 0,6.0343160812,0.901940576,-1.7560210279$

$\mathrm{H}, 0,8.3747195716,0.373530105,-2.298185169$

$\mathrm{H}, 0,9.5165676513,-1.5411772976,-1.1870622968$

$\mathrm{H}, 0,8.2985147317,-2.9261912654,0.4509675147$

$\mathrm{H}, 0,6.2566580661,-3.4495204958,1.6809964623$

$\mathrm{H}, 0,4.5795518138,-4.9275673259,2.2609821125$

$\mathrm{H}, 0,3.4668538737,-4.6182530242,3.6175535379$

$\mathrm{H}, 0,5.0919862698,-3.88693221,3.6241390582$

$\mathrm{H}, 0,-3.3936755728,-2.7990073722,-0.7861122657$

$\mathrm{H}, \mathrm{O},-4.4855186678,-1.8167743795,-1.7814906529$

$\mathrm{H}, \mathrm{O},-2.9698349658,-2.433758528,-2.4578349767$

\section{TS- $7^{\prime}$-B}

Opt a wB97XD/SDD/6-31G (d,p) in toluene (SMD model)

$\mathrm{SCF}$ Done: $\mathrm{E}(\mathrm{RwB} 97 \mathrm{XD})=-2877.60842293 \mathrm{a} \cdot \mathrm{u}$.

Zero-point correction $=0.792569$ Hartree/Particle

Sum of electronic and thermal Free Energies = -2876.924285 a.u. @ $358.15 \mathrm{~K}$

Imaginary Freq. $=-193.9170 \mathrm{~cm}^{-1}$

$\mathrm{Sp}$ a B3LYP-D3 (BJ)/def2-TZVPP in toluene (SMD model)

$\mathrm{SCF}$ Done: $\mathrm{E}(\mathrm{RB} 3 \mathrm{LYP})=-2879.52362011 \mathrm{a} \cdot \mathrm{u}$.

$\mathrm{Rh}, 0,-0.7280336197,-1.0379927442,-0.7031766871$

$0,0,-0.3853691877,1.069877176,-0.3462696635$

C, $0,-1.2736548378,1.6119511884,0.4091746399$

$\mathrm{C}, 0,-2.6444823899,1.652392084,0.0566480877$

$\mathrm{C}, 0,-0.9264282821,2.1541014122,1.7072059542$

$\mathrm{C}, 0,-3.6468853747,2.1023055126,0.9801584396$

$\mathrm{C}, 0,-1.8692574702,2.5527159004,2.6025237617$

$\mathrm{C}, 0,-3.2603705968,2.5124328162,2.2808757191$

$\mathrm{H}, 0,-1.5662627501,2.9089283957,3.5827786942$

C, $0,-5.5785130721,2.8827947538,2.8810570993$

C, $0,-5.9670326305,2.4826370127,1.5871994953$

$\mathrm{C}, 0,-5.0252060419,2.1025842026,0.6573428549$

$\mathrm{C}, 0,-4.2445556399,2.8985351601,3.2179180693$

$\mathrm{H}, \mathrm{O},-6.3297062683,3.1787368537,3.6063914584$

$\mathrm{H}, 0,-7.0199188761,2.471121991,1.3216586298$

$\mathrm{H}, \mathrm{O},-5.3337886946,1.7893783264,-0.335059596$

$\mathrm{H}, \mathrm{O},-3.9299328127,3.2094428375,4.2107264589$ 
C $, 0,-2.9457127286,1.5186216197,-1.3897479376$ $C, 0,-3.1632659196,2.6437361068,-2.1890671618$ $C, 0,-2.958741301,0.2442175677,-1.9748072791$ $C, 0,-3.3747635783,2.5130124773,-3.557226164$ $\mathrm{H}, \mathrm{O},-3.166046915,3.6267031508,-1.7268951201$ $C, 0,-3.1266702939,0.1205532699,-3.3604640789$ C, $0,-3.3400181338,1.2469602019,-4.1435115735$ $\mathrm{H}, \mathrm{O},-3.5540424988,3.3937176108,-4.1659833927$ $\mathrm{H}, \mathrm{O},-3.1153582688,-0.8659949582,-3.8135693792$ $\mathrm{H}, \mathrm{O},-3.4843394397,1.1376502868,-5.2141885965$ C $, 0,-2.3951164992,-0.7755668191,0.2373315436$ $C, 0,-2.9299370668,-0.917181008,-1.0524991037$ C, $0,-3.9483059869,-2.0011059327,-1.2605387907$ C, $0,-5.1505552489,-1.7458888014,-1.9354462622$ $C, 0,-3.7755259025,-3.2703687793,-0.6932912093$ C, $0,-6.1275193444,-2.7288818391,-2.0539874309$ $\mathrm{H}, \mathrm{O},-5.3299764464,-0.7663562359,-2.3646652289$ C, $0,-4.7521738355,-4.2532529328,-0.8078897704$ $\mathrm{H}, 0,-2.8635005466,-3.4886011222,-0.1500837389$ C, $0,-5.9329764473,-3.9891040309,-1.49582011$ $\mathrm{H}, \mathrm{O},-7.0494031829,-2.5037182973,-2.581782961$ $\mathrm{H}, 0,-4.5873250504,-5.2283529464,-0.359345207$ $\mathrm{H}, \mathrm{O},-6.6959730886,-4.7553466321,-1.5915087628$ C $, 0,-2.8773302713,-1.1535642398,1.5863841351$ $\mathrm{Cl}, 0,0.7607939989,2.2577111803,2.1362326308$ $0,0,1.1243492557,1.2731233268,-3.0919301068$ $0,0,3.4095987874,-3.1534611004,2.214832924$ $C, 0,0.2975812279,-2.70715046,0.3679628442$ C, $0,-0.2560780818,-3.1294089638,-0.8881002496$ C, $0,0.506089107,-2.507576347,-1.9390453604$ C, $0,1.4250378583,-1.6309861663,-1.3441668331$ C, $0,1.2888816312,-1.7365243553,0.1035959138$ $C, 0,2.4738294995,-0.8503045784,-2.0930415628$ C, $0,2.7406366,0.5411596816,-1.5696448468$ C, $0,2.0025413943,1.6269338133,-2.1268357595$ C, $0,2.2035623883,2.9112634938,-1.6921251497$ C, $0,3.1266190066,3.1746888458,-0.6487990008$ C, $0,3.3239935963,4.4914113256,-0.1565917408$ $C, 0,4.1886538132,4.7344623032,0.8799130212$ C, $0,4.8974735676,3.6685145529,1.4773994675$ C, $0,4.7307585844,2.3854863385,1.018806428$ $C, 0,3.8510620777,2.1042782388,-0.0588482997$ C, $0,3.6401408705,0.7764467304,-0.5545466375$ C, $0,0.1332981881,2.2133760892,-3.4528377619$ C, $0,2.1233701406,-1.0075962035,1.1208836496$ C, $0,3.6010352214,-1.2306737586,0.8892559277$ $C, 0,4.3325010379,-0.383852913,0.0855467525$ C, $0,5.720900744,-0.6348825055,-0.169117228$ C, $0,6.5011747925,0.1952921662,-1.0153683117$ C, $0,7.8283966879,-0.0745054104,-1.2387322684$ $\mathrm{C}, 0,8.4395768994,-1.1916841094,-0.6276343324$ $C, 0,7.7079619483,-2.0172648352,0.1882568522$ C, $0,6.3335369516,-1.7648710258,0.4355991004$ C, $0,5.5637686481,-2.6281810178,1.2595732244$ C, $0,4.2320240268,-2.3746160207,1.468794878$ C, $0,3.9543051542,-4.3091174377,2.8151169205$ $\mathrm{H}, \mathrm{O},-0.0091888978,-3.0390891678,1.3509447807$ $\mathrm{H}, \mathrm{O},-0.9992364689,-3.8999335973,-1.0363811611$ 
$\mathrm{H}, \mathrm{0}, \mathrm{0} .363857742,-2.6518450639,-3.0014749772$

$\mathrm{H}, 0,2.1778157287,-0.7968583633,-3.1415717199$

$\mathrm{H}, 0,3.4013718471,-1.4318521448,-2.0474273265$

$\mathrm{H}, 0,1.6406913662,3.7387914066,-2.1082657413$

$\mathrm{H}, 0,2.7663402203,5.3060915364,-0.610942726$

$\mathrm{H}, 0,4.3246418869,5.7465209543,1.2493546458$

$\mathrm{H}, 0,5.5734057825,3.865752973,2.3036794094$

$\mathrm{H}, 0,5.273196442,1.5693214443,1.4842637582$

$\mathrm{H}, \mathrm{O},-0.3970274418,2.5699456529,-2.5653811211$

$\mathrm{H}, \mathrm{O},-0.5653036528,1.6840028498,-4.1001122605$

$\mathrm{H}, 0,0.5618601133,3.0603864472,-4.0047681887$

$\mathrm{H}, 0,1.8402185013,-1.3495080123,2.1180671101$

$\mathrm{H}, 0,1.8993209418,0.058439499,1.0610609227$

$\mathrm{H}, 0,6.0339911443,1.0529582952,-1.487566871$

$\mathrm{H}, 0,8.4111947804,0.5710785787,-1.888250697$

$\mathrm{H}, 0,9.4900665947,-1.398008351,-0.8092857485$

$\mathrm{H}, 0,8.1722344629,-2.8817830164,0.6555177596$

$\mathrm{H}, 0,6.0523546927,-3.4895120883,1.7007972041$

$\mathrm{H}, 0,4.3453928083,-5.0069382609,2.0643146723$

$\mathrm{H}, 0,3.1348028026,-4.7870172707,3.3535370061$

$\mathrm{H}, 0,4.7510027302,-4.0578074679,3.5261722146$

$\mathrm{H}, 0,-2.4525868078,-0.4616726169,2.3218017828$

$\mathrm{H}, \mathrm{O},-3.9688686253,-1.123676996,1.6584717543$

$\mathrm{H}, \mathrm{O},-2.5225198371,-2.1534676633,1.8592282281$

\section{INT-8'-A}

Opt \& wB97XD/SDD/6-31G(d,p) in toluene (SMD model)

$\mathrm{SCF}$ Done: $\mathrm{E}(\mathrm{RwB} 97 \mathrm{XD})=-2877.66452321 \mathrm{a} \cdot \mathrm{u}$.

Zero-point correction $=0.795338$ Hartree/Particle

Sum of electronic and thermal Free Energies = -2876.975741 a.u. @ $358.15 \mathrm{~K}$

$\mathrm{Sp}$ @ B3LYP-D3 (BJ)/def2-TZVPP in toluene (SMD model)

$\mathrm{SCF}$ Done: $\mathrm{E}(\mathrm{RB} 3 \mathrm{LYP})=-2879.56291869 \mathrm{a} \cdot \mathrm{u}$.

$\mathrm{Rh}, 0,-0.7456796785,-0.8421256612,-0.4620865162$

$0,0,-0.2810480482,1.1566269854,0.1445088268$

C, $0,-1.2075213576,1.7541470549,0.7043999259$

$\mathrm{C}, 0,-2.6211709029,1.3006148834,0.415365677$

$\mathrm{C}, 0,-0.9759192215,2.8664246971,1.6122341711$

$\mathrm{C}, 0,-3.6730746593,1.8553609445,1.3458412612$

C, $0,-1.9809089427,3.3455614414,2.3719218421$

$\mathrm{C}, 0,-3.3429169009,2.8462411214,2.2833891853$

$\mathrm{H}, \mathrm{O},-1.7770209845,4.1364856497,3.0883864762$

C, $0,-5.6429159883,2.9293582211,3.0285741783$

$\mathrm{C}, 0,-5.9746268248,1.9632992031,2.0777176047$

C, $0,-4.998540156,1.4387227194,1.2403668057$

C, $0,-4.3339431746,3.3725242131,3.1251834066$

$\mathrm{H}, 0,-6.4056616727,3.336385759,3.6841417853$

$\mathrm{H}, \mathrm{0},-6.9999703178,1.6191837449,1.9879325046$

$\mathrm{H}, \mathrm{O},-5.2638214886,0.6929752597,0.4983150855$

$\mathrm{H}, \mathrm{O},-4.0639308675,4.1346893089,3.8507105439$

$\mathrm{C}, 0,-2.8620615199,1.7959923853,-1.0136541133$

$\mathrm{C}, 0,-3.0248791934,3.1035837927,-1.4443371552$

C, $0,-2.8902200488,0.7158277389,-1.9002365974$

C, $0,-3.2604291023,3.3351356098,-2.8003853851$

$\mathrm{H}, \mathrm{O},-2.9997462625,3.9286437121,-0.7373355782$

$\mathrm{C}, 0,-3.1343472626,0.9532353216,-3.2534530324$

C, $0,-3.3202646585,2.2630965774,-3.6920853951$ 
$\mathrm{H}, \mathrm{O},-3.4124026358,4.3486165061,-3.1578500792$ $\mathrm{H}, \mathrm{O},-3.1788999647,0.1277635059,-3.9578447444$ $\mathrm{H}, \mathrm{O},-3.5161198733,2.4522855696,-4.7436613559$ $C, 0,-2.6127345415,-0.274397311,0.2400909921$ C, $0,-2.7258263317,-0.5618838928,-1.1820724473$ $C, 0,-3.3617938098,-1.7804963885,-1.8109002007$ C, $0,-3.1254427429,-1.0772862608,1.3886375426$ C, $0,-4.2489201297,-1.9045417079,1.3036824865$ C, $0,-2.4843306939,-0.9670639654,2.6318137646$ C, $0,-4.6959060525,-2.6229931386,2.4124029765$ $\mathrm{H}, \mathrm{O},-4.7941815175,-1.9789979403,0.3692655535$ C, $0,-2.9363208718,-1.6674255354,3.7412384464$ $\mathrm{H}, 0,-1.6063463579,-0.3312290081,2.7169701916$ C, $0,-4.0437163315,-2.5094797922,3.6343875435$ $\mathrm{H}, \mathrm{O},-5.5681850706,-3.2635003299,2.3189057474$ $\mathrm{H}, 0,-2.4201784381,-1.5624198754,4.6910391365$ $\mathrm{H}, \mathrm{0},-4.3952001082,-3.064606129,4.4986598434$ $\mathrm{Cl}, 0,0.646262914,3.4671080856,1.7578811709$ $0,0,1.074428381,1.1358415929,-3.0507110872$ $0,0,3.4168505157,-3.1726875062,2.4192815187$ $C, 0,0.1536778352,-2.6047660413,0.6879395262$ $C, 0,-0.5348108504,-2.98392101,-0.5162209746$ C, $0,0.2312639132,-2.5002405425,-1.6382575302$ C, $0,1.2676265075,-1.6968619723,-1.1350060615$ C, $0,1.2118943174,-1.7571761308,0.3265681527$ C, $0,2.333392792,-1.0286889532,-1.9682404379$ C, $0,2.6844790381,0.3741192063,-1.5276036999$ C, $0,1.99008844,1.4766489745,-2.1149423545$ C, $0,2.2660332189,2.7663073158,-1.7388227853$ C, 0, 3.2391196291,3.0272141984,-0.7406312012 C, $0,3.5254663659,4.352166171,-0.3199476136$ C, 0,4.442489496,4.5945490476,0.6717049045 C, 0, 5.1151278336,3.5190910719,1.2933475322 C, 0,4.8615994268,2.2277386226,0.9023960088 C, $0,3.9247937106,1.9465801761,-0.1257966026$ $C, 0,3.6300869603,0.610970632,-0.5555047422$ C, $0,0.1909625535,2.1376867045,-3.5072224747$ C, $0,2.1367922612,-1.041797564,1.2793567454$ C, $0,3.5918432241,-1.3281507919,0.9833116037$ C, $0,4.3077481944,-0.547693216,0.1029081304$ C, $0,5.6726610415,-0.856687689,-0.2089686342$ C, $0,6.4324213178,-0.0957560005,-1.1350475549$ $\mathrm{C}, 0,7.7375918639,-0.4193625653,-1.4111779137$ C, $0,8.3460103358,-1.5237638479,-0.7748803692$ C, $0,7.6333206047,-2.2832072734,0.1184846314$ C, $0,6.2812314386,-1.9752263352,0.420157818$ C, $0,5.5294070168,-2.7688907239,1.3263796294$ C, $0,4.2182926759,-2.4616642668,1.5890229981$ C, $0,3.9576571298,-4.3167613583,3.0434572245$ $\mathrm{H}, \mathrm{O},-0.1466760333,-2.8482357991,1.6984360637$ $\mathrm{H}, 0,-1.3692962471,-3.669898722,-0.5697002462$ $\mathrm{H}, 0,0.0193617733,-2.6756489182,-2.6845771427$ $\mathrm{H}, 0,1.993332865,-1.0094155638,-3.0049425686$ $\mathrm{H}, 0,3.23677195,-1.6473711586,-1.9328325056$ $\mathrm{H}, 0,1.7379447665,3.605474486,-2.1770125763$ $\mathrm{H}, 0,2.9965226095,5.1746351424,-0.7942318342$ $\mathrm{H}, 0,4.6490036381,5.6136413196,0.9848591106$ $\mathrm{H}, 0,5.8340531874,3.7157386486,2.082607678$ 
$\mathrm{H}, \mathrm{O}, 5.3791379414,1.4049415807,1.3841244456$

$\mathrm{H}, \mathrm{O},-0.3728561698,2.5757522178,-2.6756627715$

$\mathrm{H}, 0,-0.5006591555,1.6421982079,-4.1867836165$

$\mathrm{H}, \mathrm{O}, 0.7220206646,2.9289123134,-4.0522240086$

$\mathrm{H}, 0,1.8930186355,-1.3536455931,2.2967198248$

$\mathrm{H}, 0,1.9630194858,0.0356075279,1.2154765495$

$\mathrm{H}, 0,5.9678102764,0.7528703027,-1.6259315217$

$\mathrm{H}, 0,8.3042378693,0.173756453,-2.1221953575$

$\mathrm{H}, 0,9.3791959825,-1.7726415923,-0.9979052147$

$\mathrm{H}, \mathrm{O}, 8.0953368425,-3.1380367318,0.6054617234$

$\mathrm{H}, 0,6.0133630628,-3.6215659403,1.7891092182$

$\mathrm{H}, 0,4.2874519898,-5.059052267,2.3062463119$

$\mathrm{H}, 0,3.1539959791,-4.744027559,3.6444721817$

$\mathrm{H}, 0,4.7984598845,-4.0597319866,3.700088639$

$\mathrm{H}, \mathrm{O},-3.268842131,-2.6661311617,-1.1824013937$

$\mathrm{H}, \mathrm{O},-4.4300925322,-1.6100723865,-2.0063705689$

$\mathrm{H}, \mathrm{O},-2.8874017724,-2.0079614706,-2.7708977418$

\section{INT-8'-B}

Opt a wB97XD/SDD/6-31G $(\mathrm{d}, \mathrm{p})$ in toluene (SMD model)

SCF Done: $\mathrm{E}(\mathrm{RwB} 97 \mathrm{XD})=-2877.65159021 \mathrm{a} \cdot \mathrm{u}$.

Zero-point correction $=0.795781$ Hartree/Particle

Sum of electronic and thermal Free Energies = -2876.963235 a.u. @ $358.15 \mathrm{~K}$

Sp a B3LYP-D3 (BJ)/def2-TZVPP in toluene (SMD model)

SCF Done: E (RB3LYP) $=-2879.55074375$ a.u.

$\mathrm{Rh}, 0,-0.7940062559,-0.9470520476,-0.6352976824$

$0,0,-0.3377734064,1.0964237145,-0.0458322959$

C, $0,-1.2590333325,1.6762913076,0.5412022668$

C, $0,-2.6615388144,1.1843046254,0.284239739$

C, $0,-1.0356658112,2.7835745005,1.4582192133$

$\mathrm{C}, 0,-3.7266564722,1.7579361361,1.1872818577$

C, $0,-2.0482948118,3.2647336118,2.207364527$

C, $0,-3.41078329,2.7669295805,2.109588908$

$\mathrm{H}, \mathrm{O},-1.8494216139,4.0589466567,2.9218219361$

C, $0,-5.7248103019,2.8695065524,2.8080039266$

C, $0,-6.0406631343,1.8792018938,1.8761486655$

C, $0,-5.0498095201,1.3347169628,1.0690325945$

C, $0,-4.4173571286,3.3148407833,2.918776855$

$\mathrm{H}, \mathrm{O},-6.4995955649,3.2945793036,3.4375459912$

$\mathrm{H}, \mathrm{O},-7.0647474477,1.5339207407,1.7765846873$

$\mathrm{H}, 0,-5.3001787055,0.5729610749,0.3361654919$

$\mathrm{H}, \mathrm{O},-4.1621327606,4.0948014242,3.6306317281$

C, $0,-2.9341015542,1.5644406801,-1.1683020776$

C, $0,-3.0587485659,2.8417682267,-1.6961510963$

C, $0,-2.9911133268,0.4183805176,-1.9660146259$

$\mathrm{C}, 0,-3.2509043242,2.9833035452,-3.068998695$

$\mathrm{H}, \mathrm{O},-3.0224629083,3.7134940327,-1.0477583172$

C, $0,-3.1308198016,0.5732813922,-3.3498823464$

C, $0,-3.2723690514,1.8504543022,-3.8857621807$

$\mathrm{H}, \mathrm{O},-3.374035235,3.9705903556,-3.5026786208$

$\mathrm{H}, \mathrm{O},-3.1310002285,-0.2943007098,-4.0019781513$

$\mathrm{H}, \mathrm{O},-3.3992049609,1.9655599416,-4.9584296794$

C, $0,-2.5966746495,-0.3938159716,0.2374038698$

C, $0,-2.8882318481,-0.8039076533,-1.1312857979$

C, $0,-3.7662776641,-1.9817490033,-1.4363026372$

C, $0,-4.8330690603,-1.860089687,-2.3416520774$ 
$C, 0,-3.6581460147,-3.2058458805,-0.7572146312$ C, $0,-5.7138078048,-2.9110856936,-2.5827093848$ $\mathrm{H}, \mathrm{O},-4.9985688094,-0.9224003474,-2.8579886025$ C, $0,-4.5378061252,-4.2563046364,-0.9909663004$ $\mathrm{H}, \mathrm{O},-2.8723927625,-3.3419639015,-0.0279367687$ $\mathrm{C}, 0,-5.5695222393,-4.1218999082,-1.9155277047$ $\mathrm{H}, 0,-6.5224113779,-2.7730557237,-3.2947386567$ $\mathrm{H}, 0,-4.4119583097,-5.1864456109,-0.4442316076$ $\mathrm{H}, \mathrm{O},-6.253339251,-4.943600071,-2.1043753471$ C, $0,-3.0243934793,-1.0930205892,1.51051275$ $\mathrm{Cl}, 0,0.5810796226,3.3924862093,1.6193793444$ $0,0,1.1287898424,1.3907022669,-2.9574467526$ $0,0,3.3160122952,-3.2861188778,2.2247793065$ C, $0,0.2045451844,-2.717278107,0.3935496578$ $C, 0,-0.3962055779,-3.0558927756,-0.8727826601$ $\mathrm{C}, 0,0.3745685591,-2.4375146845,-1.9151226407$ C, $0,1.3138385378,-1.5974544707,-1.2993573029$ C, $0,1.2153790537,-1.7756459674,0.1491630968$ C, $0,2.3838633409,-0.8331952369,-2.0376260793$ $\mathrm{C}, 0,2.7255688056,0.5281681334,-1.4803448885$ $\mathrm{C}, 0,2.0507867423,1.6705943887,-2.0087013928$ C, $0,2.3483214729,2.9337252852,-1.5674860256$ C, $0,3.3076436764,3.1223265065,-0.5402300294$ C, $0,3.6087855488,4.4181250906,-0.0454364519$ C, 0,4.5025003534,4.5908719753,0.9813911304 C, $0,5.1372790825,3.471527595,1.5636394714$ C, 0,4.869727109,2.2071940163,1.1003071311 $\mathrm{C}, 0,3.9577154492,1.9975600075,0.0334041419$ $\mathrm{C}, 0,3.6523103461,0.6918483706,-0.47561994$ C, $0,0.2275775854,2.4126546155,-3.3251612074$ C, $0,2.0801458973,-1.0914828494,1.1771274921$ C, $0,3.5491567459,-1.3503404415,0.9236372324$ $\mathrm{C}, 0,4.2995992287,-0.5145170784,0.1257951496$ $C, 0,5.6735656409,-0.8126339197,-0.1596178503$ $\mathrm{C}, 0,6.4704425513,0.0024242743,-1.0052793057$ $\mathrm{C}, 0,7.7826080062,-0.3125200526,-1.2574068529$ $\mathrm{C}, 0,8.3621679195,-1.4623716879,-0.6772574997$ C, $0,7.613574698,-2.27481075,0.1364093035$ C, $0,6.2540807465,-1.9758510453,0.4123647132$ C, $0,5.4673866956,-2.8241752524,1.2355068445$ C, $0,4.1498061921,-2.5261791321,1.472934459$ $\mathrm{C}, 0,3.8237720764,-4.4809707399,2.777837313$ $\mathrm{H}, 0,-0.1009117291,-3.0780313129,1.3669290501$ $\mathrm{H}, \mathrm{O},-1.1542651078,-3.8070179893,-1.0377828231$ $\mathrm{H}, \mathrm{O}, 0.2235444259,-2.5513730195,-2.9798200295$ $\mathrm{H}, 0,2.0729911764,-0.7305582195,-3.0783278135$ $\mathrm{H}, 0,3.2893540785,-1.4506754902,-2.0297124986$ $\mathrm{H}, 0,1.8415017376,3.8038736855,-1.9687267054$ $\mathrm{H}, 0,3.1085070986,5.2744176708,-0.4901316001$ $\mathrm{H}, 0,4.7199774661,5.588187518,1.3519808971$ $\mathrm{H}, 0,5.8371299396,3.6125901784,2.3814327733$ $\mathrm{H}, 0,5.3573804826,1.350838731,1.5536604083$ $\mathrm{H}, \mathrm{O},-0.279557807,2.8235634761,-2.4455660409$ $\mathrm{H}, \mathrm{O},-0.5104369365,1.9456699212,-3.9757210133$ $\mathrm{H}, 0,0.7315865465,3.2205177269,-3.8719432694$ $\mathrm{H}, 0,1.7998828101,-1.4557682534,2.1672422082$ $\mathrm{H}, 0,1.8931646001,-0.015035144,1.1552695824$ $\mathrm{H}, 0,6.0287036941,0.8852613624,-1.4549342945$ 
$\mathrm{H}, 0,8.3774183542,0.3225332532,-1.9064818583$

$\mathrm{H}, 0,9.4009010295,-1.7044156265,-0.881142117$

$\mathrm{H}, 0,8.0523644807,-3.1648059862,0.5799239394$

$\mathrm{H}, 0,5.9314115142,-3.7100940151,1.6539320714$

$\mathrm{H}, 0,4.1694275574,-5.1704462863,1.9977074555$

$\mathrm{H}, 0,2.9960000604,-4.9410697714,3.319093628$

$\mathrm{H}, 0,4.6450011225,-4.2859486884,3.4789116149$

$\mathrm{H}, 0,-2.6029075676,-0.5679083608,2.3744151797$

$\mathrm{H}, 0,-4.1134399284,-1.117714181,1.6394785761$

$\mathrm{H}, \mathrm{O},-2.6594937921,-2.117382483,1.5577811485$

\section{TS-6- $\left(S_{\mathrm{a}}, \boldsymbol{R}_{\mathrm{Rh}}\right)-\mathrm{K} 2$}

Opt d wB97XD/SDD/6-31G $(d, p)$ in toluene (SMD model)

$\mathrm{SCF}$ Done: $\mathrm{E}(\mathrm{RwB} 97 \mathrm{XD})=-2840.28990503 \mathrm{a} \cdot \mathrm{u}$.

Zero-point correction $=0.779994$ Hartree/Particle

Sum of electronic and thermal Free Energies $=-2839.616397 \mathrm{a} . \mathrm{u}$. @ $358.15 \mathrm{~K}$

Imaginary Freq. = -297.6118 $\mathrm{cm}^{-1}$

Sp a B3LYP-D3 (BJ)/def2-TZVPP in toluene (SMD model)

SCF Done: $\mathrm{E}(\mathrm{RB} 3 \mathrm{LYP})=-2842.18784582 \mathrm{a} \cdot \mathrm{u}$.

$\mathrm{Rh}, 0,-0.7012885562,-1.5042608631,0.0674789349$

$0,0,-0.1373157711,0.4218973094,0.5807582696$

C, $0,-1.0060249115,1.3750013588,0.7505881892$

C, $0,-2.119846958,1.5594868752,-0.0754024423$

$C, 0,-0.8114831557,2.3038771473,1.8307051676$

C, $0,-3.120607057,2.529230783,0.2586724733$

C, $0,-1.7185805845,3.2717776082,2.1304109692$

C, $0,-2.9145892875,3.3906721618,1.3739813438$

$\mathrm{H}, \mathrm{O},-1.5449784908,3.9440377664,2.9647046508$

C, $0,-5.0661963871,4.4552169149,1.0133239584$

$C, 0,-5.2984663975,3.5800501459,-0.0688012382$

$C, 0,-4.3589957281,2.6463828329,-0.4331643364$

$C, 0,-3.8955436047,4.351170988,1.7215118608$

$\mathrm{H}, \mathrm{O},-5.8141589225,5.1918194113,1.2894943129$

$\mathrm{H}, \mathrm{O},-6.2359037941,3.6370426874,-0.6145426554$

$\mathrm{H}, \mathrm{O},-4.5738163358,1.9730792193,-1.2552333406$

$\mathrm{H}, \mathrm{O},-3.7049647972,5.0015916278,2.5713744403$

C, $0,-2.1882339017,0.7653751396,-1.3198192359$

C, $0,-2.4540631293,1.4280378399,-2.5314035641$

C, $0,-1.9490264534,-0.6267194541,-1.367943299$

C, $0,-2.5250259858,0.7553887934,-3.7423379736$

$\mathrm{H}, 0,-2.5918227282,2.5047251592,-2.5119093994$

C, $0,-2.073907598,-1.3037707607,-2.593981646$

$C, 0,-2.347687625,-0.6278667079,-3.772371862$

$\mathrm{H}, \mathrm{O},-2.7256001642,1.3057511996,-4.6562817334$

$\mathrm{H}, \mathrm{O},-1.9755672159,-2.3831701729,-2.6148293676$

$\mathrm{H}, 0,-2.4270213104,-1.1760431252,-4.7063519318$

C, $0,-2.3405379914,-1.2009049038,1.2803200606$

C, $0,-2.8901471088,-1.4874634202,0.1557990415$

C, $0,0.2339862897,-3.1774270348,-1.0766800279$

C, $0,-0.2629738863,-3.6572697177,0.1797566677$

$C, 0,0.5099120686,-3.0423548963,1.2107784916$

C, $0,1.3942037946,-2.1153768911,0.6044776907$

C, $0,1.2405920201,-2.2159959962,-0.841365489$

C, $0,2.2972954217,-1.1703773202,1.3481620419$

C, $0,2.0316008721,-1.5050591465,-1.9118978567$

$\mathrm{H}, \mathrm{O},-0.1165227233,-3.4853176968,-2.0522731033$ 
$\mathrm{H}, \mathrm{O},-1.0273961661,-4.4103551985,0.3143356865$ $\mathrm{H}, 0,0.4002306032,-3.2015771953,2.2748728257$ $\mathrm{Cl}, 0,0.6418937359,2.1573005251,2.7902456518$ C, $0,-2.4931304183,-0.8471397579,2.6686140648$ C, $0,-1.3982877059,-0.8403823454,3.5413743817$ $C, 0,-3.7560843642,-0.4609596221,3.1479239596$ $\mathrm{C}, 0,-1.5656959461,-0.4781343259,4.8727868755$ $\mathrm{H}, 0,-0.4170676253,-1.0908256604,3.1540866096$ C, $0,-3.9140835952,-0.0962618173,4.4774737825$ $\mathrm{H}, \mathrm{O},-4.5998710056,-0.4363526345,2.4656891795$ C, $0,-2.8218267617,-0.1080391894,5.3450906301$ $\mathrm{H}, 0,-0.7082162946,-0.4683103127,5.5384240374$ $\mathrm{H}, 0,-4.8925858652,0.2054102295,4.8380344029$ $\mathrm{H}, \mathrm{O},-2.9490740663,0.1838048588,6.3829706435$ C, $0,-4.0497837662,-2.2089630483,-0.3785954436$ $C, 0,-4.3479980077,-3.4735342503,0.1437112105$ C, $0,-4.9002974533,-1.6439086177,-1.3347990311$ C, $0,-5.4723001578,-4.1665360074,-0.2926025358$ $\mathrm{H}, \mathrm{O},-3.6926893497,-3.9025823495,0.8956535297$ C, $0,-6.0277988493,-2.3359533718,-1.7609427364$ $\mathrm{H}, 0,-4.6777986644,-0.6599187307,-1.7337552274$ $\mathrm{C}, 0,-6.31309817,-3.5993653615,-1.2467664263$ $\mathrm{H}, \mathrm{O},-5.6924369514,-5.1481374164,0.1154606117$ $\mathrm{H}, 0,-6.687276039,-1.8867563206,-2.4969070927$ $\mathrm{H}, 0,-7.191803895,-4.1389418307,-1.5864090987$ C, $0,2.1076433651,-0.0089451648,-1.6985145779$ C, $0,1.130858172,0.8147010149,-2.3109873621$ C, $0,1.0858306507,2.1569312354,-2.0646243397$ C, $0,2.0150027027,2.7576668693,-1.1780851375$ C, $0,1.9503766193,4.1385834509,-0.85838722$ C, $0,2.8436241579,4.6997772534,0.0167985989$ $\mathrm{C}, 0,3.8480582962,3.901213712,0.609440344$ $C, 0,3.9358533378,2.5633864839,0.3196084745$ C, $0,3.0214806391,1.9502885549,-0.5790125756$ C, $0,3.055535408,0.5480415802,-0.8645962548$ $C, 0,3.717163111,-1.1552859393,0.8261113742$ C, $0,4.0803702599,-0.3264230282,-0.2182267559$ C, $0,5.4287420883,-0.3338036089,-0.7017194426$ C, $0,5.8489435764,0.488244301,-1.7830279589$ C, $0,7.1481822529,0.4649709364,-2.2215318688$ C, $0,8.0990966501,-0.3824836098,-1.6071483113$ C, $0,7.7237205355,-1.1924462978,-0.5671670263$ C, $0,6.3861043394,-1.1916566625,-0.0907044815$ $\mathrm{C}, 0,5.9768385585,-2.0301833684,0.9778737574$ C, $0,4.681491853,-2.011716349,1.4162213683$ $\mathrm{H}, 0,0.3173829701,2.772593887,-2.5232988568$ $\mathrm{H}, 0,1.1692737017,4.7418424788,-1.3132344709$ $\mathrm{H}, 0,2.7797527412,5.7555633009,0.2615758418$ $\mathrm{H}, 0,4.5504443063,4.3499318387,1.3052578241$ $\mathrm{H}, 0,4.7026275807,1.95618598,0.7892244555$ $\mathrm{H}, 0,5.1260525305,1.1411799587,-2.260271264$ $\mathrm{H}, 0,7.4507004159,1.1015207646,-3.0473817333$ $\mathrm{H}, 0,9.1243754057,-0.3901832051,-1.9641191445$ $\mathrm{H}, 0,8.4459588851,-1.85013803,-0.0908263736$ $\mathrm{H}, 0,6.7080597816,-2.6854307841,1.4434036217$ $\mathrm{H}, 0,2.2948572393,-1.4542217764,2.4049349883$ $\mathrm{H}, \mathrm{O}, 1.8510900136,-0.1704166453,1.2803473217$ $\mathrm{H}, 0,3.0362957932,-1.938789432,-1.9545966844$ 
$\mathrm{H}, 0,1.552203023,-1.7119143539,-2.8733360695$

$\mathrm{H}, 0,4.3757361452,-2.6554114416,2.2369826578$

$\mathrm{H}, 0,0.3901828265,0.3574917055,-2.9613387418$

\section{TS-6- $\left(R_{\mathrm{a}}, S_{\mathrm{Rh}}\right)-\mathrm{K} 2$}

Opt \& wB97XD/SDD/6-31G(d,p) in toluene (SMD model)

$\mathrm{SCF}$ Done: $\mathrm{E}(\mathrm{RwB} 97 \mathrm{XD})=-2840.28901930 \mathrm{a} \cdot \mathrm{u}$.

Zero-point correction $=0.779897$ Hartree/Particle

Sum of electronic and thermal Free Energies $=-2839.614753 \mathrm{a} \cdot \mathrm{u}$. d $358.15 \mathrm{~K}$

Imaginary Freq. $=-292.7350 \mathrm{~cm}^{-1}$

Sp \& B3LYP-D3 (BJ)/def2-TZVPP in toluene (SMD model)

SCF Done: E (RB3LYP) $=-2842.18518241 \mathrm{a} \cdot \mathrm{u}$.

$\mathrm{Rh}, 0,-0.5888596493,-1.1022388432,-0.3957600328$

$0,0,-1.2387332161,0.5247879065,-1.5315728445$

$C, 0,-2.3866141391,1.0684309513,-1.2340006801$

$C, 0,-2.7395055356,1.4216059488,0.070688494$

C, $0,-3.3378999551,1.3223316042,-2.2787309857$

C, $0,-4.0723749577,1.8415333145,0.3755515833$

$C, 0,-4.5949746537,1.7762285796,-2.0223855346$

C, $0,-5.0080085351,2.015512775,-0.6848060027$

$\mathrm{H}, \mathrm{O},-5.2993543148,1.9263442279,-2.8342911364$

$C, 0,-6.762530202,2.5755185602,0.896552562$

C, $0,-5.8509227537,2.3764936496,1.9558194377$

C, $0,-4.5475514719,2.0235134902,1.7045657481$

$\mathrm{C}, 0,-6.3424800498,2.3939588145,-0.3969148557$

$\mathrm{H}, 0,-7.7891037513,2.8580046155,1.1075343259$

$\mathrm{H}, \mathrm{O},-6.1848127146,2.4964257103,2.98231396$

$\mathrm{H}, \mathrm{O},-3.870283089,1.8629633064,2.5357470186$

$\mathrm{H}, \mathrm{O},-7.0345537971,2.5249910851,-1.224787515$

C, $0,-1.688240273,1.3125293007,1.1051401985$

C, $0,-1.4440897267,2.4006358137,1.9574384172$

$C, 0,-0.8898968753,0.1557680286,1.2597090693$

C, $0,-0.4702697845,2.3552261379,2.9459733241$

$\mathrm{H}, \mathrm{O},-2.0284497838,3.3059211646,1.8210570399$

C, $0,0.0487446819,0.1026770924,2.2997813263$

$C, 0,0.2770362003,1.1929539143,3.1253477437$

$\mathrm{H}, 0,-0.2967882431,3.2219688745,3.5760227856$

$\mathrm{H}, 0,0.5882955306,-0.8210244934,2.4771561196$

$\mathrm{H}, \mathrm{O}, 1.0303826969,1.135347192,3.9049171068$

C, $0,-2.5727224205,-1.5668944524,-0.0322806518$

$C, 0,-2.0971137399,-1.4178441943,1.1501030912$

C, $0,0.7146733573,-2.8647986968,-0.107026234$

C, $0,0.0432736737,-3.0235131497,-1.3650174852$

C, $0,0.412453405,-1.9332191092,-2.1715570233$

C, $0,1.3862243714,-1.1252705993,-1.4484947946$

$C, 0,1.6232655011,-1.7552261644,-0.2036093908$

$C, 0,2.0410543788,0.1101254173,-2.009747057$

C, $0,2.2948850589,1.1909754407,-0.9784455833$

C, $0,1.3234813656,2.2110683646,-0.8116561872$

C, 0,1.4811309274,3.1853212215,0.1329157096

C, $0,2.6188118096,3.1953105967,0.979094086$

C, $0,2.7855764336,4.1784351257,1.9889138384$

C, 0,3.8761194406,4.1562513742,2.8192520255

C, $0,4.85190143,3.1430723737,2.6752068364$

C, $0,4.7186333553,2.1798714793,1.7074110084$

C, 0, 3.6020844407,2.1784180433,0.8272715196 
$C, 0,3.4202578588,1.1744165469,-0.178098001$ C, $0,2.6599918638,-1.3918123661,0.8278948467$ C, $0,4.0227137852,-1.1964124353,0.1974531694$ C, $0,4.3878207175,0.0430685761,-0.2925349694$ C, $0,5.6600266177,0.2178792519,-0.9227818804$ C, $0,6.0724050859,1.4682143602,-1.4584483729$ C, $0,7.2983820241,1.6054551968,-2.0576655901$ C, $0,8.1774275564,0.5018282447,-2.1562668178$ C, $0,7.8056768224,-0.7184974164,-1.6547501789$ C, $0,6.542867557,-0.8933434045,-1.0296312104$ C, $0,6.1349107109,-2.1508329634,-0.5138070058$ C, $0,4.9096810044,-2.2956018293,0.0776240361$ $\mathrm{H}, 0,0.654693233,-3.5432277533,0.734551629$ $\mathrm{H}, 0,-0.6778649852,-3.7918650422,-1.6078348742$ $\mathrm{H}, 0,0.0353241317,-1.7020230002,-3.1589671522$ $\mathrm{H}, 0,1.3846624821,0.5058808074,-2.7894002034$ $\mathrm{H}, 0,2.9794125202,-0.1819859349,-2.495141987$ $\mathrm{H}, 0,0.7224659275,3.9518018204,0.2601529551$ $\mathrm{H}, 0,2.0255106964,4.9481667579,2.094131037$ $\mathrm{H}, 0,3.9933733422,4.9120076266,3.5899305419$ $\mathrm{H}, \mathrm{O}, 5.7118408887,3.1276194597,3.3379211048$ $\mathrm{H}, 0,5.4707207078,1.4040384553,1.6091202181$ $\mathrm{H}, 0,2.7037968618,-2.193077055,1.5718221883$ $\mathrm{H}, 0,2.3692877256,-0.4785774017,1.3518638023$ $\mathrm{H}, 0,5.401626413,2.3181819286,-1.3880223344$ $\mathrm{H}, 0,7.5973246894,2.5679875001,-2.461141433$ $\mathrm{H}, 0,9.1449879133,0.6248719485,-2.6330147918$ $\mathrm{H}, 0,8.4724795836,-1.5733644402,-1.7296334623$ $\mathrm{H}, 0,6.8111167718,-2.9973223077,-0.5965436324$ $\mathrm{Cl}, 0,-2.8510736366,0.9897060102,-3.9255284642$ $C, 0,-3.7148728939,-1.8150339025,-0.8737243364$ C, $0,-3.5767845959,-2.2783610848,-2.1876993638$ C, $0,-4.9977222312,-1.5301092169,-0.3767493209$ C, $0,-4.7001130895,-2.4757279932,-2.9815353886$ $\mathrm{H}, 0,-2.5831215723,-2.4563336662,-2.5846051423$ C, $0,-6.1138204178,-1.7223795959,-1.1787652536$ $\mathrm{H}, 0,-5.1027099787,-1.1329192506,0.6277468901$ C, $0,-5.9698797313,-2.197704732,-2.4815555643$ $\mathrm{H}, \mathrm{O},-4.5819158692,-2.8303242439,-4.0007082722$ $\mathrm{H}, 0,-7.0990018197,-1.4856543622,-0.7891848789$ $\mathrm{H}, 0,-6.844392444,-2.3392656725,-3.1090607599$ $\mathrm{C}, 0,-2.2223828149,-1.9824302651,2.4978003522$ C, $0,-2.1526748834,-3.3714154336,2.6556915213$ C, $0,-2.4680736501,-1.1754204229,3.6133037318$ C, $0,-2.3199824665,-3.9436231975,3.9126802209$ $\mathrm{H}, 0,-1.9694551145,-3.9939200464,1.7849688709$ C, $0,-2.6448948748,-1.7518617342,4.8654112101$ $\mathrm{H}, \mathrm{O},-2.5223090132,-0.0988184129,3.4911317849$ $C, 0,-2.565506294,-3.134673831,5.0192084526$ $\mathrm{H}, 0,-2.2613120023,-5.0216014611,4.0267153877$ $\mathrm{H}, 0,-2.8439637566,-1.1190133521,5.7245475008$ $\mathrm{H}, \mathrm{O},-2.6975458345,-3.5805009296,6.0002269474$ $\mathrm{H}, 0,0.4279680852,2.173553367,-1.4224593132$ $\mathrm{H}, 0,4.6039864265,-3.2626876635,0.4683846648$ 